\title{
Manufacturing Demonstration Facility: Roll-to-Roll Processing
}

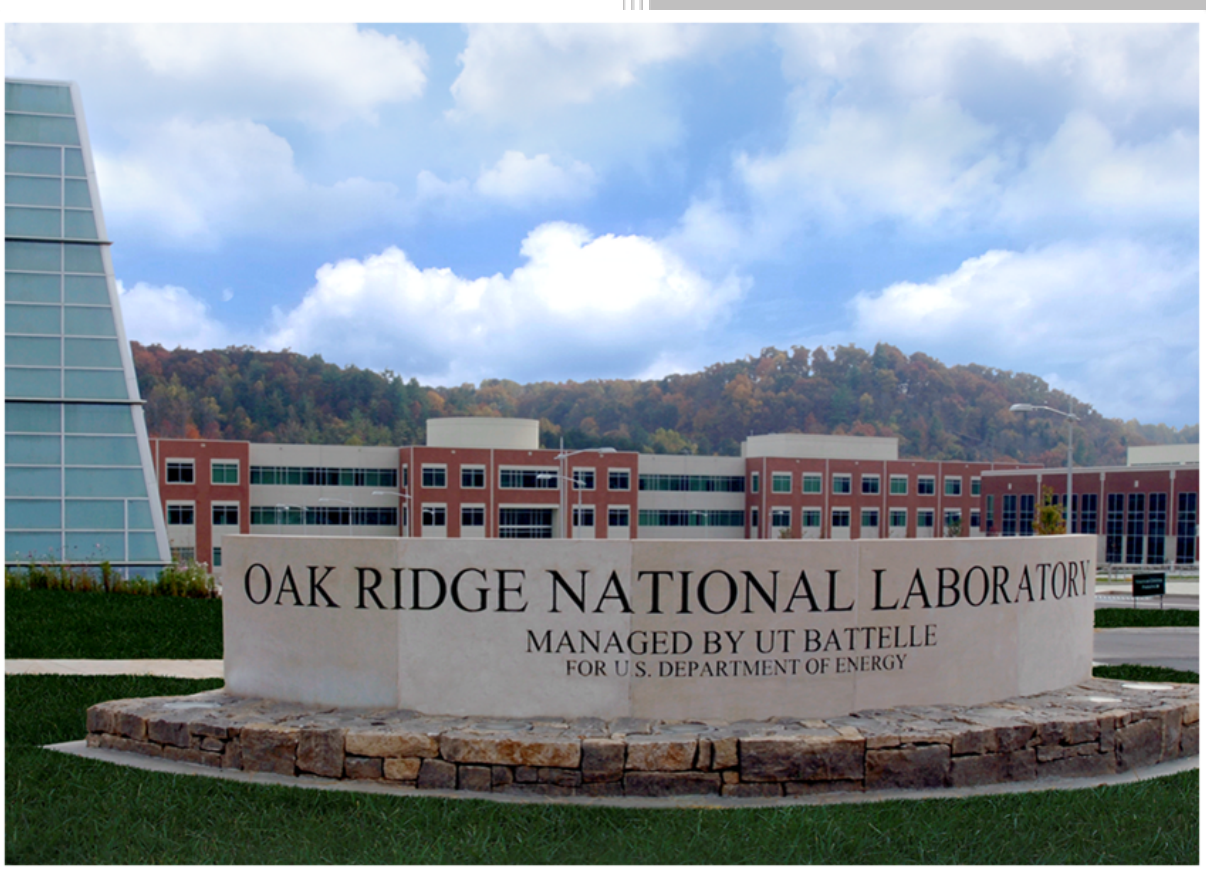

\section{Approved for public release;} distribution is unlimited
Panos G. Datskos Pooran C. Joshi Chad E. Duty Frederick A. List Beth L. Armstrong Ilia N. Ivanov Christopher B. Jacobs David E. Graham Ji Won Moon

August 5, 2015 


\title{
DOCUMENT AVAILABILITY
}

Reports produced after January 1, 1996, are generally available free via US Department of Energy (DOE) SciTech Connect.

Website http://www.osti.gov/scitech/

Reports produced before January 1,1996, may be purchased by members of the public from the following source:

\author{
National Technical Information Service \\ 5285 Port Royal Road \\ Springfield, VA 22161 \\ Telephone 703-605-6000 (1-800-553-6847) \\ TDD 703-487-4639 \\ Fax 703-605-6900 \\ E-mail info@ntis.gov \\ Website http://www.ntis.gov/help/ordermethods.aspx
}

Reports are available to DOE employees, DOE contractors, Energy Technology Data Exchange representatives, and International Nuclear Information System representatives from the following source:

Office of Scientific and Technical Information

PO Box 62

Oak Ridge, TN 37831

Telephone 865-576-8401

Fax 865-576-5728

E-mail reports@osti.gov

Website http://www.osti.gov/contact.html

This report was prepared as an account of work sponsored by an agency of the United States Government. Neither the United States Government nor any agency thereof, nor any of their employees, makes any warranty, express or implied, or assumes any legal liability or responsibility for the accuracy, completeness, or usefulness of any information, apparatus, product, or process disclosed, or represents that its use would not infringe privately owned rights. Reference herein to any specific commercial product, process, or service by trade name, trademark, manufacturer, or otherwise, does not necessarily constitute or imply its endorsement, recommendation, or favoring by the United States Government or any agency thereof. The views and opinions of authors expressed herein do not necessarily state or reflect those of the United States Government or any agency thereof. 
Advanced Manufacturing Office

\title{
MANUFACTURING DEMONSTRATION FACILITY: ROLL-TO-ROLL PROCESSING
}

\author{
Author(s) \\ Panos G. Datskos \\ Pooran C. Joshi \\ Chad E. Duty \\ Frederick A. List \\ Beth L. Armstrong \\ Ilia N. Ivanov \\ Christopher B. Jacobs \\ David E. Graham \\ Ji Won Moon
}

Date Published: June, 2015

\author{
Prepared by \\ OAK RIDGE NATIONAL LABORATORY \\ Oak Ridge, TN 37831-6283 \\ managed by \\ UT-BATTELLE, LLC \\ for the \\ US DEPARTMENT OF ENERGY \\ under contract DE-AC05-00OR22725
}





\section{CONTENTS}

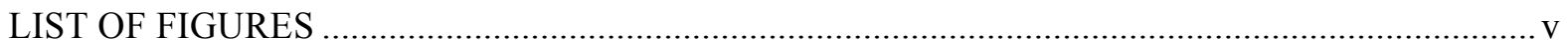

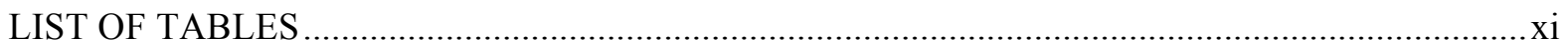

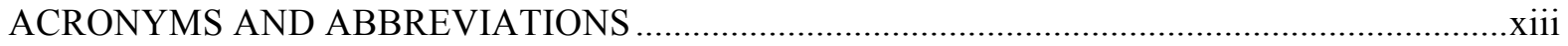

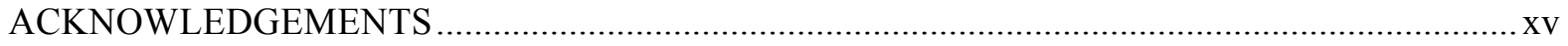

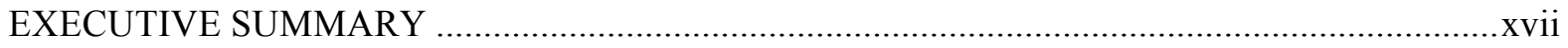

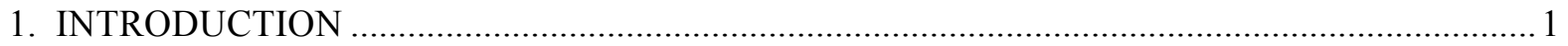

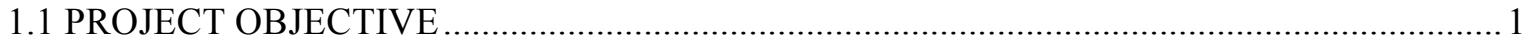

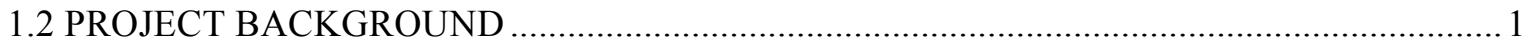

2. TASK 1: FUNCTIONALIZATION OF ZN COMPLEXES FOR OPTOELECTRONICS.............. 2

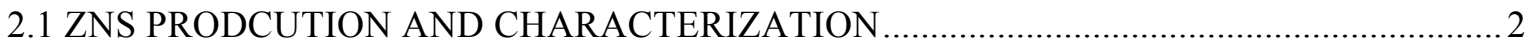

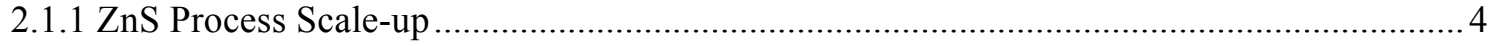

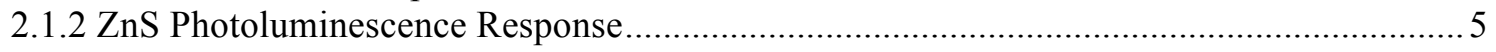

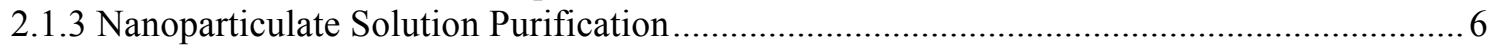

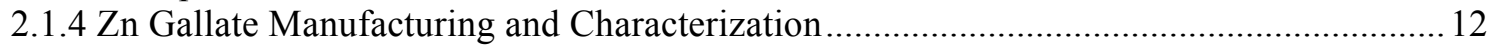

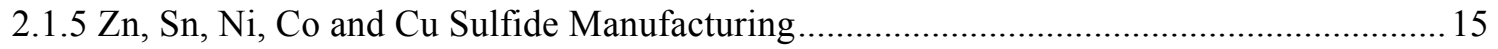

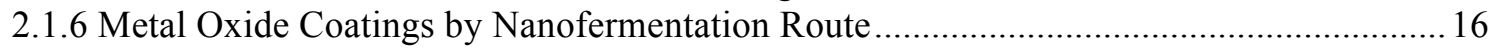

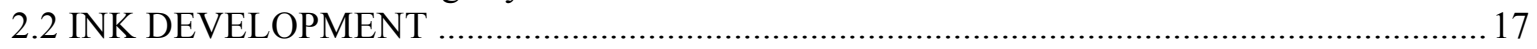

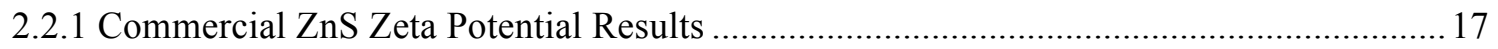

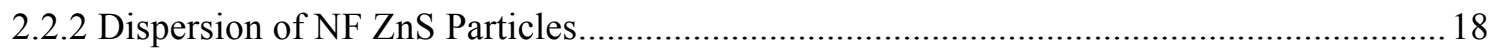

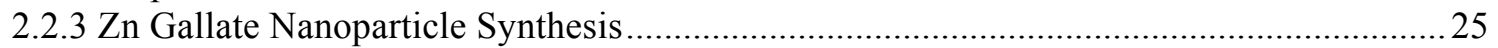

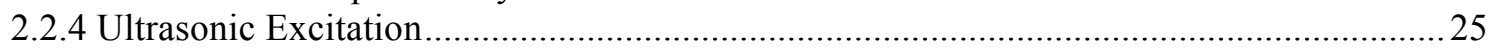

2.2.5 Oxidation of NF metal sulfide $(\mathrm{ZnS}, \mathrm{NiS}, \mathrm{SnS}, \mathrm{CoS}$, and $\mathrm{Cu})$ particles............................26

2.2.6 Oxidation of NF metal sulfide $\left(\mathrm{SnS}, \mathrm{CoS}_{2}, \mathrm{Ni}_{3} \mathrm{~S}_{4}\right.$, and $\left.\mathrm{CuS}\right)$ particles .............................27

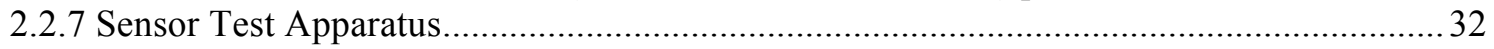

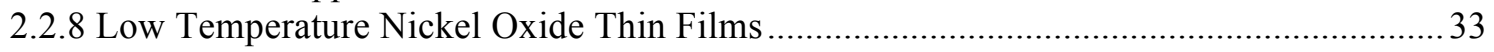

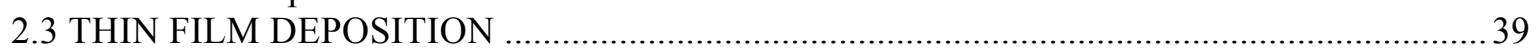

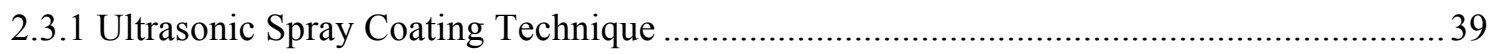

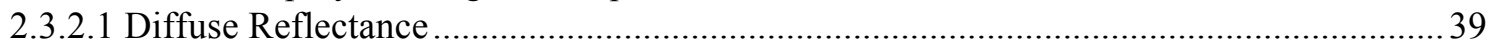

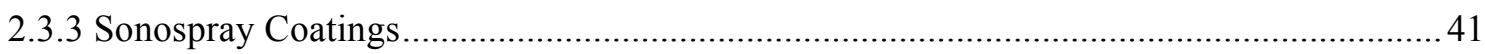

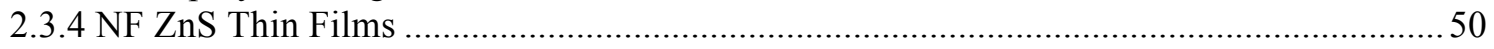

2.3.5 Preparation of the ZnO NFNP target for low temperature senor deposition ......................54

2.4 THIN FILM ANNEALING AND CHARACTERIZATION - ZN GALLATE COATINGS .. 54

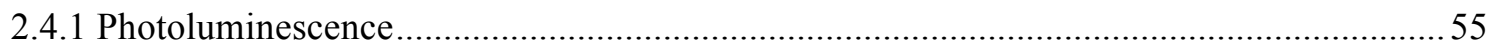

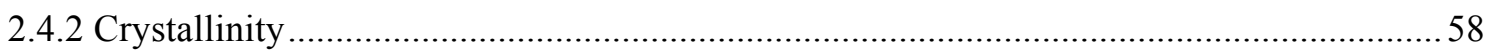

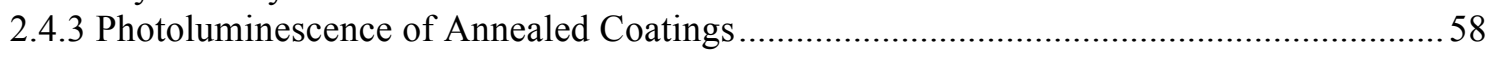

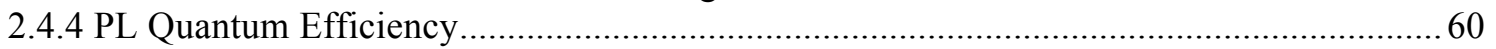

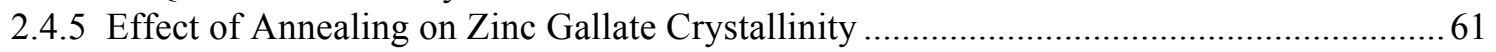

2.4.6 Photoluminescence of Zinc Gallate Powder Made by Nanofermentation ...........................62

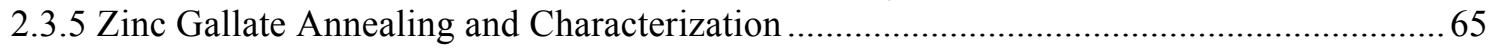

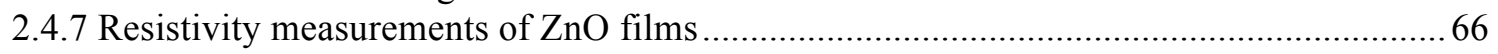

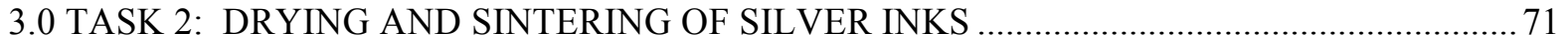

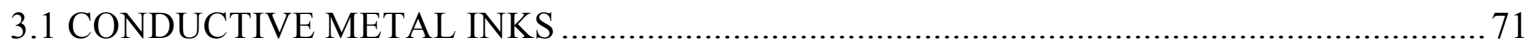

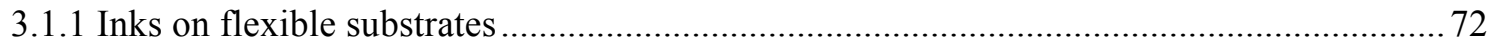

3.1.2 Sintering and electrical performance of ink jet printed materials .................................... 73

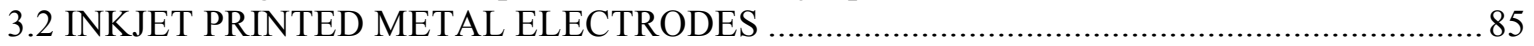

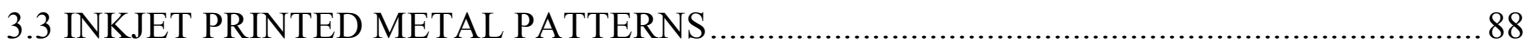

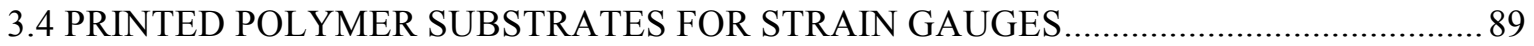

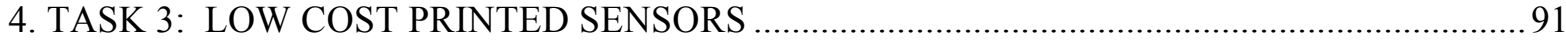




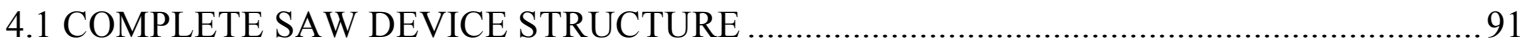

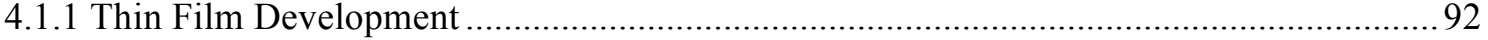

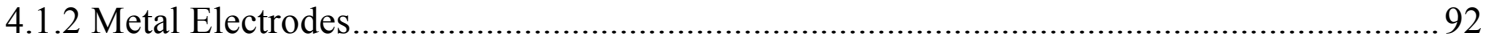

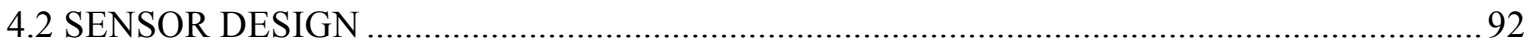

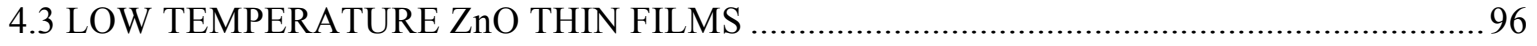

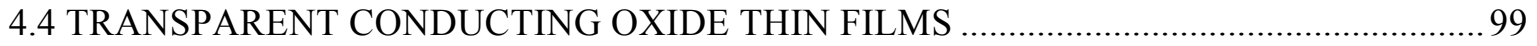

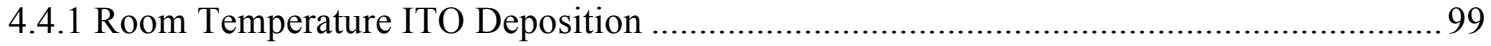

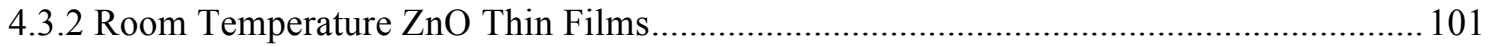

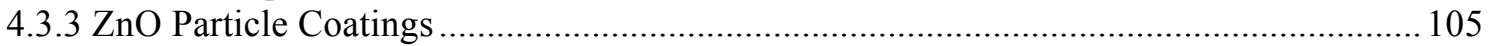

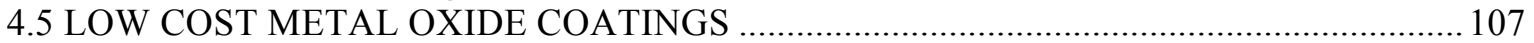

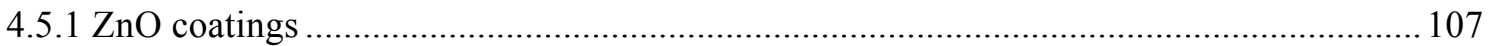

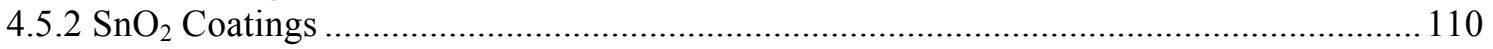

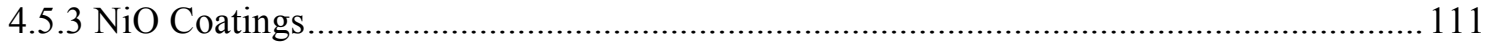

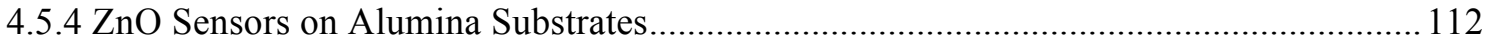

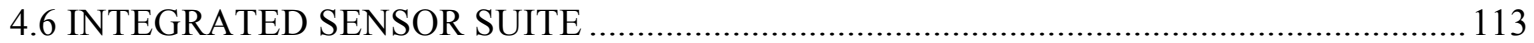

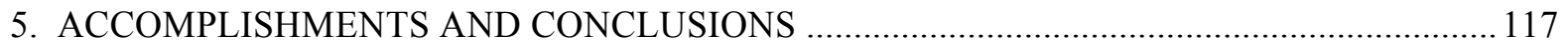

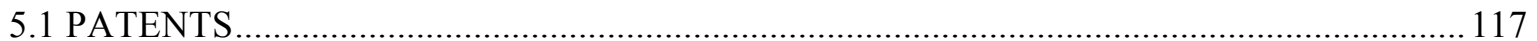

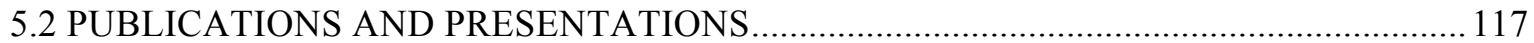

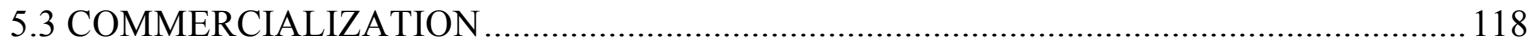

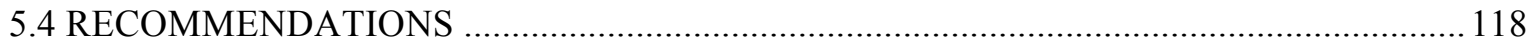




\section{LIST OF FIGURES}

Figure 1.1. General steps in the development of NF Zn complexes for optoelectronic applications.....2

Figure 1.2. Various NF nanoparticle batches investigated. ........................................................... 3

Figure 1.3. The impact of incubation time on nanoparticle growth and microstructure. ...................... 3

Figure 1.4. The impact of single dosing, thiosulfate concentration, and increased incubation time on $\mathrm{ZnS}$ nanoparticle growth and crystallinity.

Figure 1.5. (a) Setup for process scale up, and (b) x-ray diffraction patterns indicating that phase formation kinetics are well-maintained for the scaled up process.......................................................5

Figure 1.6. The impact of shorter incubation time on the (a) PL response for the batches shown in the figure, and (b) crystallinity of ZnS nanoparticles for batch number ZNS120515 ............................5 Figure 1.7. X-ray diffraction patterns of $\mathrm{ZnS}$ nanoparticles with estimated particle size of (A) $7.4 \pm$ $0.2 \mathrm{~nm}$ and (B) $7.9 \pm 0.2 \mathrm{~nm}$.

Figure 1.8. Optical density measurement of $\mathrm{ZnS}$ nanoparticle solutions synthesized with various nutrient sources.

Figure 1.9. X-ray diffraction pattern of $\mathrm{ZnS}$ nanoparticles produced with glucose (top), xylose (middle), and pyruvate (bottom

Figure 1.10. X-ray diffraction patterns of $\mathrm{ZnS}$ nanoparticles produced (A) abiotically and (B) biotically, demonstrating an increase in particle size from 4.6 to $9.5 \mathrm{~nm}$.

Figure 1.11. Fourier transfer infrared (FTIR) spectroscopy of abiotic (top) versus biotic (bottom) production of $\mathrm{ZnS}$, showing reduced organic bonding for abiotic production.

Figure 1.12. $\mathrm{ZnS}$ average agglomerate size after post recovery treatment with the various surfactants.

Figure 1.13. (right) $\mathrm{ZnS}$ average agglomerate size for various in-situ versus post recovery treatments.

Figure 1.14. ZnS agglomerate size Comparison study for in-situ treatment according to dosing........ 10 Figure 1.15. FT-IR spectroscopy and XPS measurement of the surface of NP ZnS formed by Thermoanaerobacter X513. (a): FT-IR spectra of the NP ZnS using various buffer systems; (b), XPS spectrum showing $\mathrm{C}-\mathrm{O}$ and $\mathrm{C}=\mathrm{O}$ bonding; (c), XPS spectrum showing amine $\left(-\mathrm{NH}_{2}\right)$ and protonated

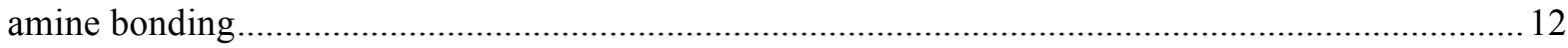

Figure 1.16. Scale up of NanoFermented phosphor production with scale factor of $100 \ldots \ldots \ldots \ldots \ldots \ldots . . .12$ Figure 1.17. XRD patterns from representative RGB Zn-gallate phosphor before post-treatment..... 13 Figure 1.18. X-ray diffraction patterns for each of the RGB color phosphors produced in the 24L

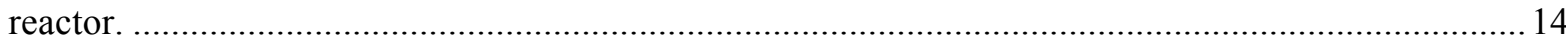

Figure 1.19. NanoFermented zinc gallate precipitates.......................................................... 15

Figure 1.20. X-ray diffraction patterns of zinc gallates using various $([\mathrm{Zn}]+[\mathrm{Mn}]) /[\mathrm{Ga}]$ ratios......... 15 Figure 1.21. (a) Examples of samples of metal sulfides $\left(\mathrm{SnS}, \mathrm{Ni}_{3} \mathrm{~S}_{4}, \mathrm{CoS}_{2}\right.$, and $\mathrm{CuS}$ ) produced in our 24 liter reactor. These will be used to fabricate thin films metal oxide gas sensors. (b) $\mathrm{SnS}$ and $\mathrm{Ni}_{3} \mathrm{~S}_{4}$ samples.

Figure 1.22. Freeze drying condensed zinc sulfide sample at $>50 \mathrm{~g}$ amount from the 100 liter reactor.

Figure 1.23. The latest batch of ZnS NF-nanoparticles produced in the Low Temperature Materials

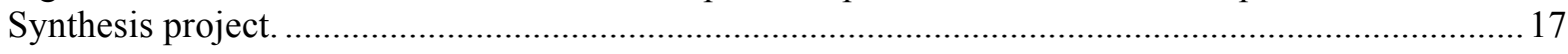

Figure 1.24. Relation between the zeta potential and $\mathrm{pH}$ of the ink using polyethyleneimine........... 18

Figure 1.25. Sedimentation Curve for Additions of $600 \mathrm{MW}$ PEI in an Aqueous Solution of 1 weight

$\% \mathrm{NF} Z \mathrm{ZnS}$.

Figure 1.26. Sedimentation Curve for Additions of 1200 MW PEI in an Aqueous Solution of 1

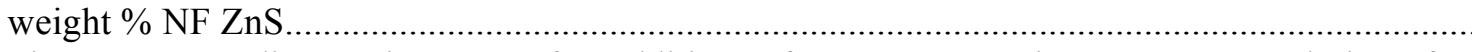

Figure 1.27. Sedimentation Curve for Additions of 1800 MW PEI in an Aqueous Solution of 1 weight $\%$ NF ZnS...... 
Figure 1.28. Weight loss cures of NF ZnS solutions in air with and without the use of the dispersant

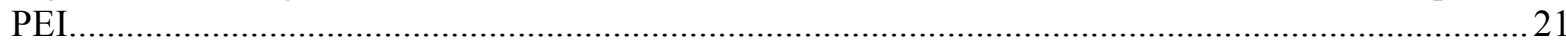

Figure 1.29. Weight loss cures of NF $\mathrm{ZnS}$ solutions in argon with and without the use of the

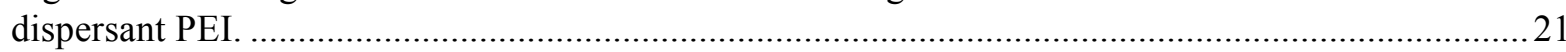

Figure 1.30. Zeta potential curves of commercial and NF ZnS powders. ......................................2 21

Figure 1.31. Sedimentation curve for alternative dispersion approaches for $\mathrm{NF} \mathrm{ZnS}$ in an aqueous

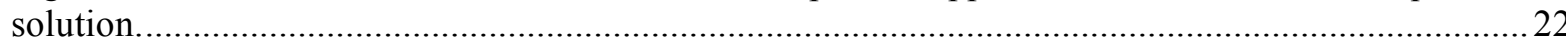

Figure 1.33. The $\mathrm{ZnS}$ was converted to $\mathrm{ZnO}$ with oxidation in $\mathrm{O}_{2}$ atmosphere at $800^{\circ} \mathrm{C}$ for 1 hour.. 27 Figure 1.34. The x-ray diffraction patterns showing the impact of $\mathrm{O}_{2}$ annealing on the metal oxide phase formation for (a) $\mathrm{SnS}$, (b) $\mathrm{CoS}_{2}$, (c) $\mathrm{Ni}_{3} \mathrm{~S}_{4}$, and (d) $\mathrm{CuS}$ particles. ..........................................28 Figure 1.35. The impact of multiple anneals at $800^{\circ} \mathrm{C}$ on $\mathrm{ZnO}$ phase formation.............................29 Figure 1.36. Samples produced from the Low temperature synthesismaterials project. The material was $>50 \mathrm{~g}$ of $\mathrm{SnS}$ and was oxidized to $\mathrm{n}$-type $\mathrm{SnO}_{2}$ and used for gas sensing................................... 30 Figure 1.37. The $\mathrm{SnS}$ was converted to $\mathrm{SnO}_{2}$ with oxidation in $\mathrm{O}_{2}$ atmosphere at $800^{\circ} \mathrm{C}$ for 1 hour. 31 Figure 1.38. The Ni3S4 was converted to $\mathrm{NiO}$ with oxidation in $\mathrm{O} 2$ atmosphere at $800^{\circ} \mathrm{C}$ for 1 hour. A pure $\mathrm{NiO}$ phase was produced after annealing at $800^{\circ} \mathrm{C}$. .31 Figure 1.39. Particle size distribution of $\mathrm{NiO}$ nanoparticles that were processed to produce a printable

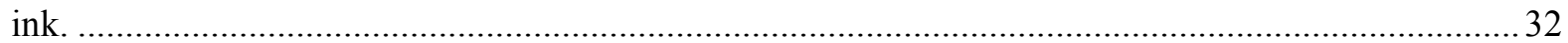

Figure 1.40. Background image of the Environmental Chamber. The inset shows the screen printed $\mathrm{ZnO}$ transistor on the surface of $\mathrm{Si} / \mathrm{SiO} 2$ wafer tested in the chamber............................................. 32 Figure 1.41. (a) Process conditions and (b) deposition rate of rf sputtered $\mathrm{NiO}$ thin films as a function

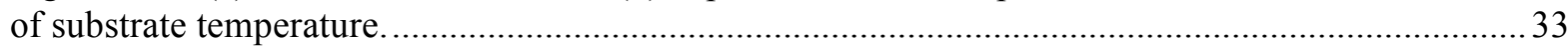
Figure 1.42. The x-ray diffraction patterns of $\mathrm{NiO}$ thin films deposited on (a) $\mathrm{Si}$ and (b) quartz substrates. 34 Figure 1.43. (a) Surface morphology and (b) SEM cross section of NiO thin films deposited on $\mathrm{Si}$

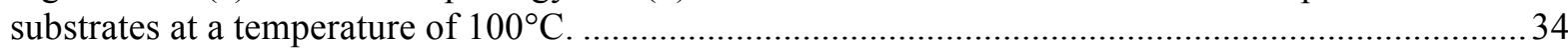
Figure 1.44. (a) Optical transmittance and (b) reflectance of $\mathrm{NiO}$ thin films as a function of process

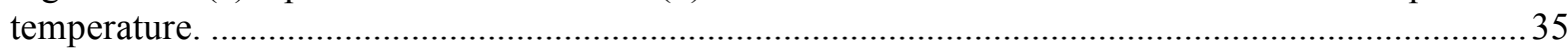

Figure 1.45. The optical bandgap of $\mathrm{NiO}$ thin films as a function of process temperature..................36 Figure 1.46. (a) Refractive index and (b) extinction coefficient of $\mathrm{NiO}$ thin films as a function of

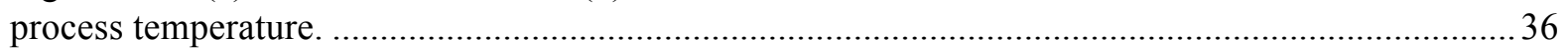
Figure 1.47. (a) Carrier concentration and (b) Hall mobility of $\mathrm{NiO}$ thin films as a function of process

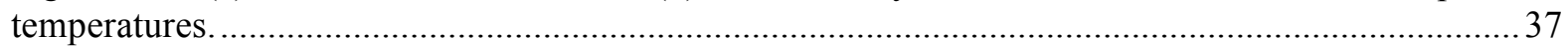
Figure 1.48. The impact of photonic curing on the structural characteristics of $\mathrm{NiO}$ thin films

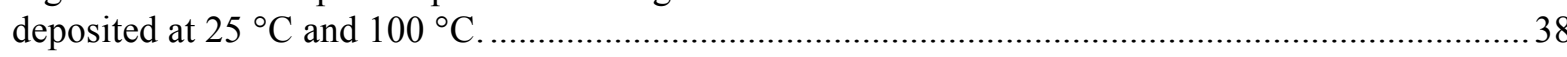
Figure 1.49. Sono-spray deposition system for nanoparticle thin film processing (L) and PerkinElmer Lambda 900 spectrophotometer with integrating sphere attachment (R) .............................. 40 Figure 1.50. a) The measured diffuse reflectivity from samples of nanocrystalline $\mathrm{ZnS}$ and CdS. b) the values of the Kubelka-Munk $\alpha / \mathrm{S}$ coefficient, determined from the reflectivity measurements..... 40 Figure 1.51. The photoluminescence spectra from the $\mathrm{CdS}$ and $\mathrm{ZnS}$ nanocrystalline samples. .........41 Figure 1.52. The $\mathrm{ZnS}$ thin film coating development in terms of (a) solvent/substrate interaction and

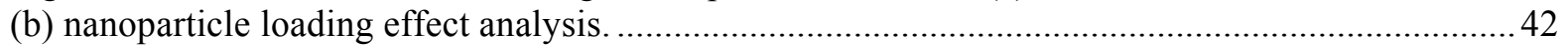
Figure 1.53. (a) Optical transmittance and (b) thin film uniformity as analyzed using 450nm LED... 42 Figure 1.54. Thermal gravimetric analysis of PEI based $\mathrm{ZnS}$ nanoparticle inks.............................. 43 Figure 1.55. Optical images of the $\mathrm{ZnS}$ thin films deposited quartz substrates: (a) as-deposited, (b) annealed at $800^{\circ} \mathrm{C} / 30$ minutes. Figure 1.56. Surface morphology of $\mathrm{ZnS}$ nanoparticle thin films: (a) as-deposited, (b) annealed at

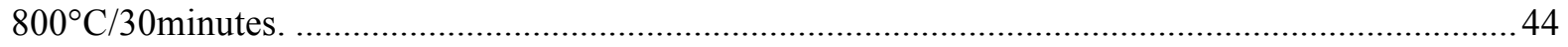
Figure 1.57. Diffused transmittance of $\mathrm{ZnS}$ nano-particle thin films deposited on quartz substrates. . 45 Figure 1.58. X-ray diffraction patterns of $\mathrm{ZnS}$ nanoparticle thin films as a function of annealing temperature 
Figure 1.59. The FTIR spectra of (L) ZnS thin films and (R) various NF ZnS nanoparticle samples. 46 Figure 1.60. Photoluminescence of various sizes of nanoparticles in solution. ….............................. 47

Figure 1.61. Photoluminescence of consolidated nanoparticle thin films. ....................................... 48

Figure 1.62. The PL response of as-deposited and annealed NF ZnS particles................................50

Figure 1.63. The XRD patterns of as-deposited and annealed NF ZnS particles $(9.5 \mathrm{~nm}) \ldots \ldots \ldots \ldots \ldots . .50$

Figure 1.64. The impact of annealing on the (a) PL response and (b) crystallinity of NF ZnS thin

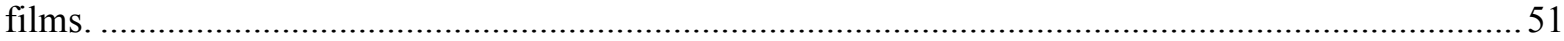

Figure 1.65. The typical PL response of as-deposited NF ZnS nanoparticles.................................51

Figure 1.66. The XRD patterns of the as-deposited NF ZnS nanoparticle (3.1-9.5nm) thin films. .....51

Figure 1.67. The NF ZnS thin films did not show any color change after annealing in an oxygen atmosphere as was observed after annealing in Ar atmosphere. ...................................................52

Figure 1.68. The impact of annealing in a pure oxygen atmosphere on the crystallinity and oxidation

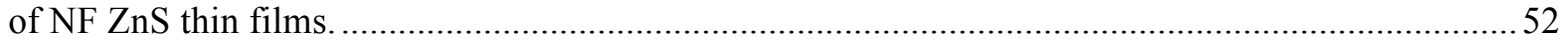

Figure 1.69. Photoluminescence spectrum with no sample (blue) and with a sample (red) in place.. 53 Figure 1.70. (a) - Raw photoluminescence data for $\mathrm{ZnO}$ sample, and (b) - Quantum efficiency data

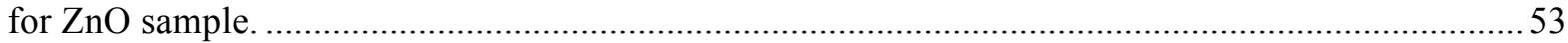

Figure 1.71. Flow chart of the sample preparation using a pressed target made from nanofermented

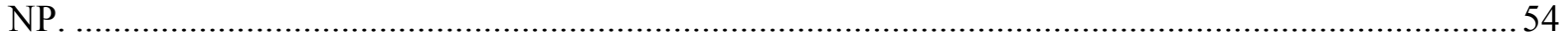

Figure 1.72. The X-ray diffraction patterns of the $\mathrm{ZnGa} 2 \mathrm{O} 4 / \mathrm{Al} 2 \mathrm{O} 3$ coatings annealed at $950^{\circ} \mathrm{C}$.... 55 Figure 1.73. The $\mathrm{X}$-ray diffraction patterns of the $\mathrm{ZnGa}_{2} \mathrm{O}_{4} / \mathrm{Al}_{2} \mathrm{O}_{3}$ coatings annealed at various temperatures: (a) $900^{\circ} \mathrm{C}$, (b) $950^{\circ} \mathrm{C}$, and (c) $1000^{\circ} \mathrm{C}$. The unlabeled peaks are due to the substrate...55 Figure 1.74. The PL response of various $\mathrm{ZnGa}_{2} \mathrm{O}_{4}$ coatings annealed at $900^{\circ} \mathrm{C}$ : (a) full spectrum $(330$ $850 \mathrm{~nm})$, (b) expanded view below $600 \mathrm{~nm}$. Sample details: $\bullet$ Co(1\%) replacing Zn, $\bullet$ Mn (4\%) replacing $\mathrm{Ga}, \bullet \mathrm{Cr}(1 \%)$ replacing $\mathrm{Ga}, \bullet \mathrm{Mn}(4 \%)$ replacing $\mathrm{Zn}$ (freeze dried with Gallium Chloride, -Mn(4\%) replacing Zn (freeze dried with Gallium Nitrate Hydrate) .56 Figure 1.75. The effect of annealing temperature on the PL response of doped $\mathrm{ZnGa} 2 \mathrm{O} 4(1 \% \mathrm{Cr}$

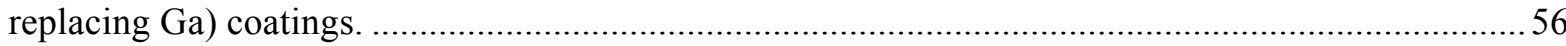
Figure 1.76. The effect of forming gas anneal on the PL response of doped $\mathrm{ZnGa}_{2} \mathrm{O}_{4}(\mathrm{Mn}(4 \%)$ replacing Ga) coatings annealed at (a) $950^{\circ} \mathrm{C}$ and (b) $1000^{\circ} \mathrm{C}$. 57 Figure 1.77. Various dopants under investigation for optical emission control for phosphor applications. 58

Figure 1.78. The $\mathrm{x}$-ray diffraction patterns of the $\mathrm{ZnGa}_{2} \mathrm{O}_{4} / \mathrm{Al}_{2} \mathrm{O}_{3}$ coatings annealed at various temperatures: (a) $900-1100^{\circ}$ anneal in oxygen atmosphere, (b) $1100^{\circ} \mathrm{C}$ anneal in air and oxygen

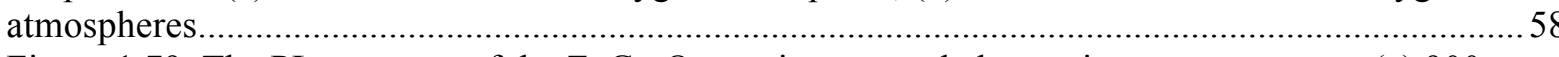
Figure 1.79. The PL response of the $\mathrm{ZnGa}_{2} \mathrm{O}_{4}$ coatings annealed at various temperatures: (a) 900 $1100^{\circ}$ anneal in oxygen atmosphere, (b) $1100^{\circ} \mathrm{C}$ anneal in air and oxygen atmospheres. .59 Figure 1.80. The impact of forming gas anneal on PL response of the $\mathrm{ZnGa}_{2} \mathrm{O}_{4}$ coatings processed at various temperatures: (a) $900-1100^{\circ}$ anneal in oxygen atmosphere, (b) $1100^{\circ} \mathrm{C}$ anneal in air and oxygen atmospheres.

Figure 1.81. Photoluminescence from 4 different zinc gallate samples, and the photoluminescence

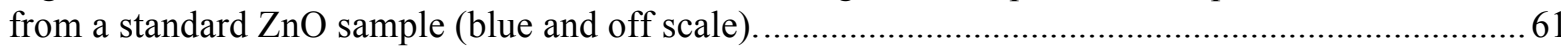

Figure 1.82. The impact of sample reflectivity on the collected PL excitation signal. ......................61

Figure 1.83. Photoluminescence of Zinc Gallate taken in the quantum efficiency configuration....... 61

Figure 1.84. X-ray diffraction patterns of annealed zinc gallate particles with various $([\mathrm{Zn}]+[\mathrm{Mn}]) /[\mathrm{Ga}]$ ratios.

Figure 1.85. Photoluminescence spectra from the trichromatic phosphor using 4 different excitation wavelengths.

Figure 1.86. Photoluminescence from the various ZGT samples when illuminated using various wavelengths from the $\mathrm{HgXe}$ lamp. Illumination using the $254 \mathrm{~nm}$ wavelength showed no observable PL, so it is not shown. .64

Figure 1.87. Comparison photoluminescence of the trichromatic phosphor and ZGT131120E 
( $\mathrm{Zn}_{1.1} \mathrm{Ga}_{2}$ ) using (a) $313 \mathrm{~nm}$ excitation from a $\mathrm{HgXe}$ lamp and (b) $325 \mathrm{~nm}$ excitation from the HeCd65

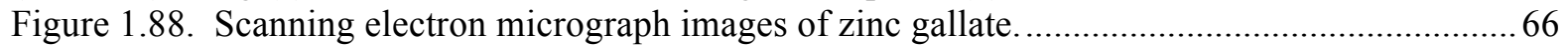

Figure 1.89. Cross sectional SEM images of PLD-deposited $\mathrm{ZnO}$ films ion $\mathrm{SiO}_{2} / \mathrm{Si}$ substrates. ....... 67 Figure 1.90. In-plane impedance spectra of PLD-deposited $\mathrm{ZnO}$ films ion $\mathrm{SiO}_{2} / \mathrm{Si}$ substrates as a function of frequency.

Figure 1.91. UV-Vis diffuse reflectance as a function of wavelength for PLD $\mathrm{ZnO}$ films, the reference and nanofermented deposited on $\mathrm{Si} / \mathrm{SiO}_{2}$ substrate.

Figure 2.1. General steps in the development and evaluation of the conductive metal inks for flexible

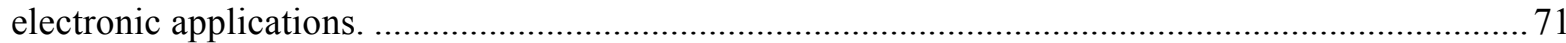

Figure 2.2. Drying results for alcohol-based silver ink on Ultem substrate. .................................. 73

Figure 2.3. Optical micrographs showing typical multi-coat print quality for each substrate..............75

Figure 2.4. Stylus profilometer results showing a) cross-sectional area and b) thickness of sintered

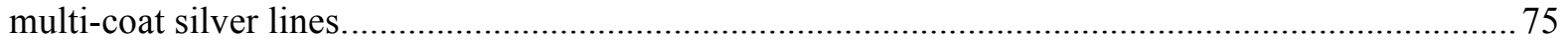

Figure 2.5. Optical micrographs showing typical print quality for each ink/substrate combination....77

Figure 2.6. Optical micrographs showing typical print quality for each substrate............................ 78

Figure 2.7. Electrical resistance for paper- and polymer-based substrates........................................ 79

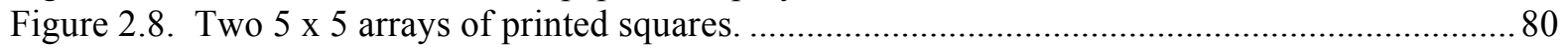

Figure 2.9. Surface profile of six lines cured using different UV light intensities............................ 80

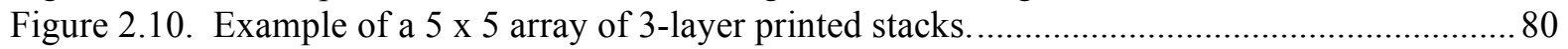

Figure 2.11. Images of a silver strain gauge printed directly on the FDM photopolymer substrate.

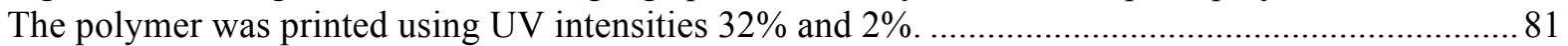

Figure 2.12. Silver resistive strain gauge printed on an FDM substrate. Inset shows higher

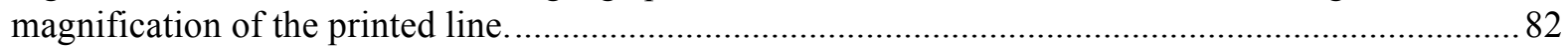

Figure 2.13. Resistance as a function of sintering time for the cured inks................................... 83

Figure 2.14. Resistance as a function of sintering time and pulse frequency rate for the cured inks.. 83 Figure 2.15. A prototype embedded strain gauge. Inkjet printed silver between two 1-mm thick FDM

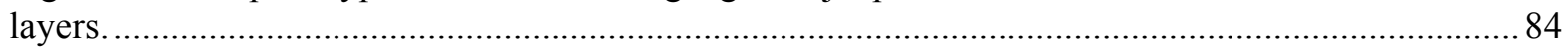
Figure 2.16. The three step process used to embed circuitry in printed structures............................ 84 Figure 2.17. Examples of embedded complex circuit geometries in printed structures.....................85 Figure 2.18. (a) PulseForge 3300 Photonic curing system and (b) DMP-2831 materials printer used for the development of silver electrode patterns on flexible plastic substrates. ................................. 85 Figure 2.19. The impact of photonic curing on the electrical conductivity of printed silver electrodes.

Figure 2.20. Impacts of photonic curing and conventional hot-plate sintering methods on the electrical

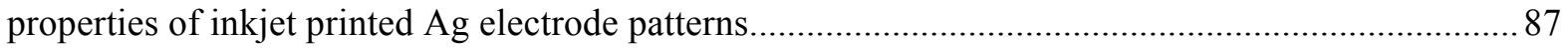

Figure 2.21. Inkjet printed interdigitated electrode patterns on flexible PET substrates.................... 88 Figure 2.22. Printed Ag test structures to evaluate resistance scaling: (a) Rectangular and (b) Spiral.

Figure 2.23. Additive printed polymer substrates for strain testing.

Figure 2.24. Polishing of the printed structures with several grit polish levels................................. 89

Figure 2.25. A close up of the wrinkled structure of the polishing of printed structures. .................. 90 Figure 3.1. (a) SAW Delay line structure with two transducers (the electrode widths $=19.8$ um for 40 $\mathrm{MHz}$ operation on LiNbO3), (b) A simple single frequency sensor with two tracks, each having a reflector which will produce a reflection at a different time delay...................................................91

Figure 3.2. Room temperature Ag thin film patterns deposited on plastic substrates by e-beam

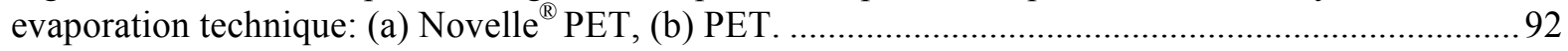

Figure 3.3. Representative 7-sensor element RF-SAW structure................................................ 93

Figure 3.4. Photomicrograph of "reference" RF-SAW device. ....................................................... 93

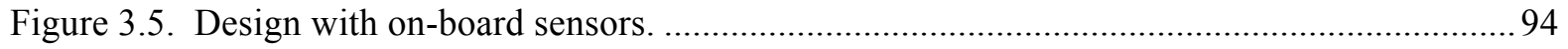

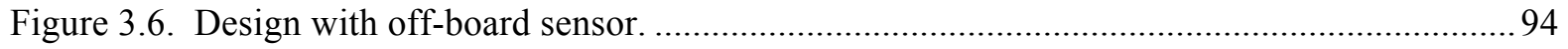

Figure 3.7. Broadband frequency and temporal response of the tested RF-SAW............................94 
Figure 3.8. RF-SAW "readings" for configuration with 5 elements.

Figure 3.9. Annotated photomicrograph of an RF-SAW device capable of operation at high

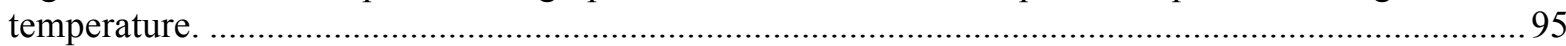

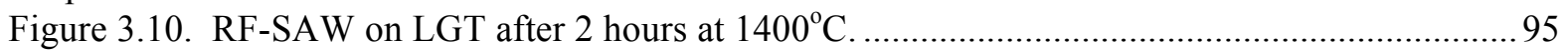

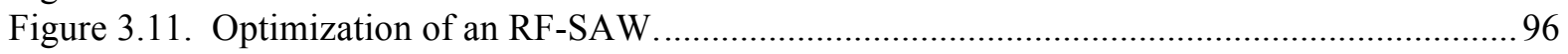

Figure 3.12. Properties of sputtered $\mathrm{ZnO}$ thin film processed at $200^{\circ} \mathrm{C}$ : (a) crystallinity, (b) optical n-k

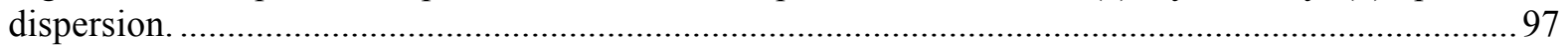

Figure 3.13. The effect of process temperature on the (a) deposition rate, and (b) crystallinity of $\mathrm{ZnO}$

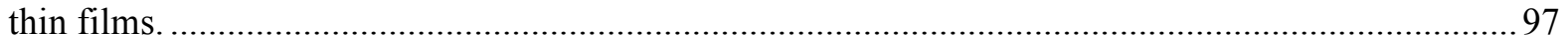

Figure 3.14. The impact of process temperature on the (a) optical transmittance, and (b) n-k

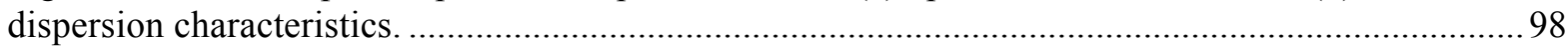

Figure 3.15. The optical transmittance of the room temperature deposited ITO thin films: (a)

transmittance of ITP/PI thin films, (b) prototypes on quartz and plastic substrates........................... 99

Figure 3.16. The impact of low thermal budget pulse thermal processing on the sheet resistance of

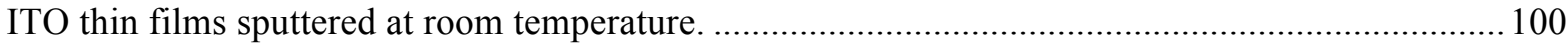

Figure 3.17. The impact of pulse thermal processing on the electrical performance of room temperature processed ITO thin films.

Figure 3.18. Room temperature deposited $\mathrm{ZnO}$ thin films: (a) thin film on inkjet printed Ag electrode

pattern, (b) x-ray diffraction pattern showing c-axis orientation.

101

Figure 3.19. The impact of pulse thermal processing on the crystallinity of room temperature

processed $\mathrm{ZnO}$ thin films.

Figure 3.20. The impact of pulse thermal processing on the current-voltage (I-V) characteristics of

room temperature processed $\mathrm{ZnO}$ thin films.

Figure 3.21. UV sensor with interdigitated Ag electrode pattern: (a) electrodes on top, (b) electrodes

underneath $\mathrm{ZnO}$ film.

Figure 3.22. (a) ZnO UV sensor with IDE pattern, (b) Photo-response under 365nm illumination. . 103 Figure 3.23. The dark-current characteristics of as-deposited and PTP processed ZnO thin films. The films were deposited on polyimide substrates at room temperature.

Figure 3.24. The photo response characteristics of as-deposited and PTP processed ZnO thin films.

The films were deposited on polyimide substrates at room temperature.

Figure 3.25. Screen-printed $\mathrm{ZnO}$ coatings: (L) x-ray diffraction patterns, (R) coating on inkjetprinted electrode pattern on plastic substrate.

Figure 3.26. (a) Normalized absorbance of as produced NF-nanoparticles. The nanoparticles tend to aggregate, have solutions that are not stable, they are optically scattering and the photo-luminescence comes form high energy states (surface defects). (b) For the stabilized NF- nanoparticles there is no observable light scattering. 106

Figure 3.27. TEM images of (a) unmodified and (b) ligand stabilized NF-nanoparticles clusters... 106 Figure 3.28. Current-voltage (I-V) response of Pt electrodes deposited on alumina substrates.......107

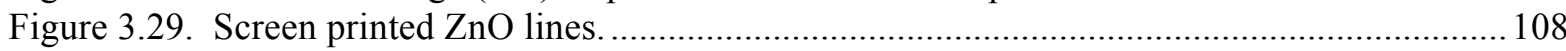
Figure 3.30. Surface and depth profile of $\mathrm{ZnO} / \mathrm{Pt} / \mathrm{Al} 2 \mathrm{O} 3$ structures as analyzed by optical

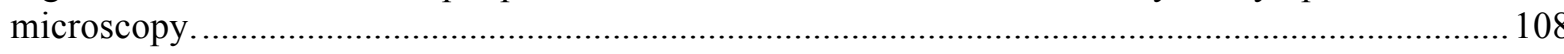
Figure 3.31. A scanning electron micrograph showing large grain growth in NF particle based $\mathrm{ZnO}$ coatings. 108

Figure 3.32. Current-voltage (I-V) characteristics of $\mathrm{ZnO}$ coatings sintered at various temperatures.

Figure 3.33. Impact of the forming gas annealing on the $I-V$ characteristics of (a) NF ZnO particle

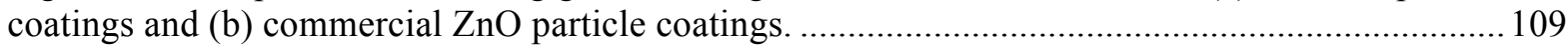
Figure 3.34. $\mathrm{SnO}_{2}$ screen printed between two Pt pads. ............................................................. 110 Figure 3.35. Pealing signs of the Pt layer (left) and the $\mathrm{SnO}_{2}$ layer (right) after annealing at $1000^{\circ} \mathrm{C}$.

Figure 3.36. Current as a function of applied voltage for two annealing temperatures: $800^{\circ} \mathrm{C}$ and 
$1,100^{\circ} \mathrm{C}$. Because the film developed discontinuities after annealing at $1000^{\circ} \mathrm{C}$ the conductivity could not be measured.

Figure 3.37. Current-voltage (I-V) characteristics of $\mathrm{NiO}$ coatings sintered at $1100{ }^{\circ} \mathrm{C}$.

Figure 3.38. $\mathrm{ZnO}$ sensor made from $1 \mathrm{~mm}$ wide strip of screen printed $\mathrm{ZnO}$ on Alumina substrate.

The electrodes were screen printed Pt $100 \mu \mathrm{m}$.

Figure 3.39. Response of $\mathrm{ZnO}$ thin film sensor to relative humidity under UV activation (a) as a function of UV illumination and (b) as a function of sensor temperature. The humidity response was

measured at $35 \mathrm{C}$ and $95 \% \mathrm{RH}$.

Figure 3.40. Response of $\mathrm{ZnO}$ thin film sensor as a function of temperature.

Figure 3.41. (a) Screen capture of the Labview based multi-channel software designed for tests of sensors manufactured from nanofermented nanoparticles, and (b) low cost processor platform for mobile sensing which allows to power up to 5 sensors is being developed for the implementation of the NF sensors.

Figure 3.42. Frequency dependent electrical properties (presented in the form of Nyquist plot of imaginary and real impedance) of $\mathrm{ZnO}$ thin film sensors prepared using pulse laser deposition. The green and blue colored curves are data for reference $\mathrm{ZnO}$ samples and the black and red curves are measurements for the nanoferemented $\mathrm{ZnO}$.

Figure 3.43. Model used to fit the measured data comprised by a combination of $\mathrm{R}_{\mathrm{S}}, \mathrm{L}_{1}$ and two parallel $\mathrm{RC}$ circuits, $\mathrm{R}_{1}, \mathrm{C}_{1}$ and $\mathrm{R}_{2}, \mathrm{C}_{2}$, connected in series.

Figure 3.44. Oxygen response of screen printed $\mathrm{ZnO}$ sensor prepared from $\mathrm{ZnS}$ nanoferemented nanoparticles. 


\section{LIST OF TABLES}

Table 1.1. Nutrients evaluated for NanoFermentation of $\mathrm{ZnS}$ to reduce organic content................... 6

Table 1.2. Experiments performed to prepare the wet and dried nanoparticle phosphors................... 12

Table 1.3. Agglomerate Size Results for the Zinc Gallate Study .................................................... 13

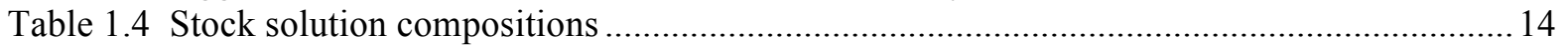

Table 1.5 Particle Size Results for NF ZnS Dispersant Screening Study ........................................23

Table 1.6 Particle Size Results for NF ZnS In-Situ Dispersant Study...............................................2

Table 1.7 Particle Size Results for NF ZnS- PEG Concentration Study ............................................24

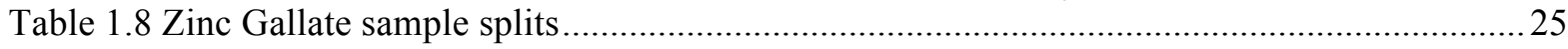

Table 1.9 The impact of $\mathrm{O}_{2}$ annealing on the metal oxide phase formation for (a) $\mathrm{SnS}$, (b) $\mathrm{CoS}_{2}$, (c)

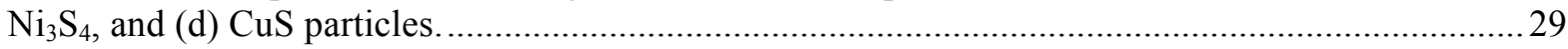

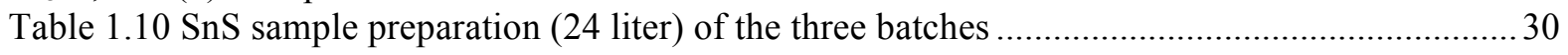

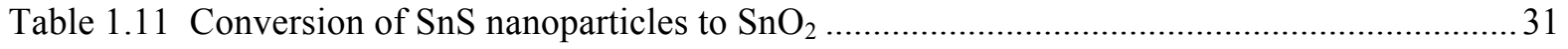

Table 1.12 Samples splits for low thermal budget photonic curing investigation............................... 38

Table 1.13 Data for photoluminescence of nanoparticles in solution for Figure 1.60 ........................46

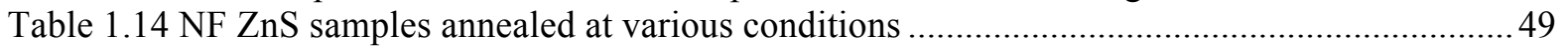

Table 1.15 Zinc Gallate sample splits for annealing investigation ..................................................58

Table 1.16 Zinc Gallate sample splits for PL investigation. All samples were annealed in $\mathrm{O}_{2}$

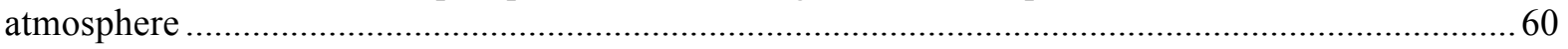

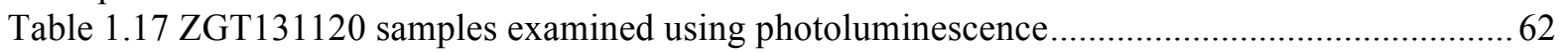

Table 1.18 Electrical performance of NF ZnO films prepared using pulse laser deposition................ 68

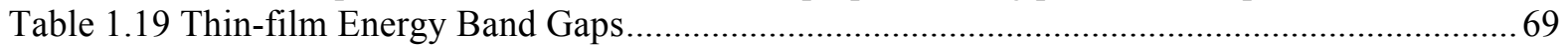

Table 2.1. Results for furnace sintering of inkjet printed silver ink. ........................................... 74

Table 2.2. Results for PTP sintering of inkjet printed silver ink ................................................... 74

Table 2.3. Results for PTP and furnace sintering of multi-coat screen printed silver ink..................76

Table 2.4. Results for furnace sintering of screen printed silver ink. ..............................................77

Table 2.5. Results for PTP sintering of screen printed silver ink ................................................ 78

Table 2.6. Results for inkjet printing and furnace sintering on paper and polymer substrates............79

Table 2.7. Pulsed photonic curing of inkjet silver nanoparticles .................................................. 82

Table 3.1. Various metal oxide thin film configurations under investigation for the flexible electronic

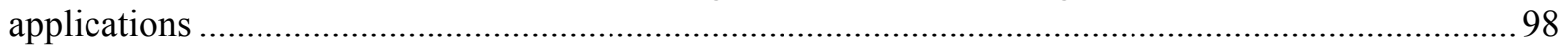

Table 3.2. Room temperature deposition of ITO thin films by pulsed de sputtering technique..........99

Table 3.3. Summary of the electrical properties of $\mathrm{ZnO}$ films prepared by pulse laser deposition. . 115 


\section{ACRONYMS AND ABBREVIATIONS}

$\begin{array}{ll}\text { ABS } & \text { Acrylonitrile Butadiene Styrene } \\ \text { AMO } & \text { Advanced Manufacturing Office } \\ \text { CATS } & \text { Center for Advanced Thin-film Systems } \\ \text { CAD } & \text { Computer Aided Drafting } \\ \text { CIGS } & \text { Copper Indium Gallium Sulfide } \\ \text { Cm } & \text { Centimeter } \\ \text { CNMS } & \text { Center for Nanophase Materials Science } \\ \text { CW } & \text { Continuous Wave } \\ \text { DOE } & \text { Department of Energy } \\ \text { EERE } & \text { Office of Energy Efficiency and Renewable Energy } \\ \text { FCC } & \text { Face Centered Cubic } \\ \text { FDM } & \text { Fused Deposition Modelling } \\ \text { FFT } & \text { Fast Fourier Transform } \\ \text { FGA } & \text { Forming Gas Annealing } \\ \text { FTIR } & \text { Fourier Transfer InfraRed } \\ \text { GLY } & \text { Glycine } \\ \text { IDE } & \text { InterDigitated Electronics } \\ \text { IEP } & \text { IsoElectric Point } \\ \text { ITO } & \text { Indium Tin Oxide } \\ \text { I-V } & \text { Current-Voltage } \\ \text { kHZ } & \text { KiloHertz } \\ \text { KW } & \text { KiloWatt } \\ \text { L } & \text { Liter } \\ \text { LED } & \text { Light Emitting Diode } \\ \text { LGT } & \text { Langatate } \\ \text { MHZ } & \text { Megahertz } \\ \text { mM } & \text { micromole } \\ \text { MW } & \text { Molecular Weight } \\ \text { NF } & \text { NanoFermentation } \\ \text { nM } & \text { NanoMole } \\ \text { nm } & \text { nanometer } \\ \text { NP } & \text { Nanoparticle } \\ \text { NTA } & \text { Nitrilotriacetic acid } \\ \text { ORNL } & \text { Oak Ridge National Laboratory } \\ \text { PEG } & \text { Polyethylene glycol } \\ \text { PEI } & \text { Polyethyleneimine } \\ \text { PET } & \text { Polyethylene Terephthalate } \\ \text { PI } & \text { Polyimide } \\ \text { pL } & \text { Picoliter } \\ \text { PL } & \text { PhotoLuminesence } \\ \text { PLD } & \text { Pulse Laser Deposition } \\ \text { PTP } & \text { Pulsed Thermal Processing } \\ \text { QE } & \text { Quantum Efficiency } \\ \text { RF } & \text { Radio Frequency } \\ \text { RF-SAW } & \text { Radio Frequency Surface Acoustic Wave } \\ \text { RFID } & \text { Radio-frequency Identification } \\ & \end{array}$




$\begin{array}{ll}\text { RGB } & \text { Red, Green, Blue } \\ \text { RH } & \text { Relative Humidity } \\ \text { SEM } & \text { Scanning Electron Microscope } \\ \text { TEM } & \text { Transmission Electron Microscope } \\ \text { TCO } & \text { Transparent Conducting Oxide } \\ \text { TGA } & \text { Thermal Gravimetric Analysis } \\ \text { UV } & \text { UltraViolet } \\ \text { XPS } & \text { X-Ray Photoelectron Spectroscopy } \\ \text { XRD } & \text { X-Ray Diffraction } \\ \text { ZGT } & \text { Zinc Gallate }\end{array}$




\section{ACKNOWLEDGEMENTS}

This Oak Ridge National Laboratory (ORNL) Manufacturing Demonstration facility (MDF) project was funded by the US Department of Energy (DOE), Office of Energy Efficiency and Renewable Energy (EERE) Advanced Manufacturing Office (AMO). This project was managed as CPS Agreement Number: 24764. The lead organization was the Oak Ridge National Laboratory. The project duration was from January 23, 2012 through March 31, 2015. Other project partners included: Novacentrix, Stratasys and Cabot Corporation. 
xvi 


\section{EXECUTIVE SUMMARY}

This Manufacturing Demonstration Facility (MDF) roll-to-roll processing project provided an excellent opportunity to investigate a number of advanced manufacturing approaches to achieve a path for low cost devices and sensors. Critical to this effort is the ability to deposit thin films at low temperatures using nanomaterials derived from nanofermentation (NF). The overarching goal of this project was to develop roll-to-roll manufacturing processes of thin film deposition on low-cost flexible substrates for electronics and sensor applications. This project utilized ORNL's unique Pulse Thermal Processing (PTP) technologies coupled with non-vacuum, low temperature deposition techniques, ORNL's clean room facility, slot dye coating, drop casting, spin coating, screen printing and other equipment including a Dimatix ink jet printer and a large-scale Kyocera ink jet printer.

This roll-to-roll processing project had three main tasks: 1) develop and demonstrate zinc-based optoelectronic sensors using low cost nanoparticulate structures manufactured in a related MDF Project using nanofermentation techniques, 2) evaluate the use of silver based conductive inks developed by project partner NovaCentrix for electronic device fabrication, and 3) demonstrate a suite of low cost printed sensors developed using non-vacuum deposition techniques which involved the integration of metal and semiconductor layers to establish a diverse sensor platform technology.

In this project we have developed non-vacuum, large-scale deposition and processing techniques for nanoparticle-based inks and pastes that reduce cost and energy requirements associated with processing of thin film electronics. We have demonstrated controllable assembly of multilayer (up to 15) nanofermented sulfide nanoparticles on areas up to $1 \mathrm{ft} x 1 \mathrm{ft}$ with a scalable technology with direct transfer of deposition parameters to roll-to-roll processing. The nanoparticles of $\mathrm{Zn}$ based complexes were produced by a nanofermentation process, which was optimized to achieve the optimal nanoparticle size and distribution. Zn complexes were synthesized and evaluated in terms of optical properties and performance. We used X-ray Diffraction (XRD) to optimize the NF process for Zn complex synthesis. We performed photoluminescence (PL) measurements that helped establish the optical absorption and emission characteristics for optoelectronic applications. PTP was instrumental in processing non-vacuum low temperature deposition by taking advantage of short exposure time radiant heating (energy flux in excess of $20 \mathrm{~kW} / \mathrm{cm}^{2}$, heating rates up to $600,000^{\circ} \mathrm{C} / \mathrm{s}$ ) over a large uniform exposure are $\left(1,000 \mathrm{~cm}^{2}\right)$.

The development of appropriate inks is critical for high quality thin films for electronics and sensor applications. In the project we pursued ink development using a variety of solvents, additives, modifiers, dispersants, and nanoparticles in order to produce thin and thick films on appropriate substrates. We deposited thin films using sonospray deposition and PTP to anneal the thin films, and then evaluated the PTP treatment in terms of the microstructure, optical, and electrical properties of thin films.

The sensors we have developed in this project can operate at $0.5 \mathrm{~V}$ compared to $5 \mathrm{~V}$ required for conventional sensors. The sensor can be activated using ultraviolet (UV) light compared to conventional sensors that require $400^{\circ} \mathrm{C}$ for activation. Use of UV light is more energy efficient compared to using a high temperature system (see Figure 3.39(a)). The UV activation process improves sensor response by a factor of five, and the sensor response is directly proportional to the exposure time (amount of material). For the baseline sensor, the impedance was reduced by $55 \mathrm{kOhm}$ under UV illumination, leading to a permanent change, and a $2 \mathrm{x}$ improvement in sensor impedance response to a single pulse. The sensor kinetics were 212 seconds with UV activation and 23 seconds partial recovery time $\left(\mathrm{CO}_{2} / \mathrm{H}_{2} \mathrm{O}\right)$. 
Zinc-based opto-electronic sensors using low cost nanofermented nanoparticules can be commercially viable depending on the desired performance. For example, the results from this project indicate that the potential for such devices is high. Furthermore, the development of conductive inks is an important component for any electronics system and particularly important for realizing low cost printed sensors and establishing a sensor platform technology. 


\section{INTRODUCTION}

\subsection{PROJECT OBJECTIVE}

The main objective of this project was to develop roll-to-roll manufacturing of thin film electronics on low-cost flexible substrates using ORNL's unique Pulse Thermal Processing (PTP) technologies coupled with non-vacuum low temperature deposition techniques. An additional objective of the project was to develop non-vacuum, large scale deposition and processing techniques for nanoparticle-based inks and pastes that reduced cost and energy requirements associated with processing of thin film electronics. The work performed was divided into three tasks:

1) Functionalization of $\mathrm{Zn}$ Complexes for Optoelectronics. This involved the development and demonstration of low-cost, phosphor materials and coatings for optoelectronic applications using zinc complexes produced by NanoFermentation (NF), deposited by low-temperature non-vacuum techniques, and sintered using Pulse Thermal Processing (PTP) technologies.

2) Drying and Sintering of Silver Inks. The main focus of this task was the evaluation of aqueous and solvent based conductive inks developed by NovaCentrix for electronic device applications. Our goal was to deposit silver inks on plastic substrates by roll-to-roll compatible inkjet printing and screen-printing techniques and use the PulseForge tool to combine the drying and sintering processes into a single pass for high throughput commercial applications - potentially reducing processing time by 2-3 orders of magnitude.

3) Low Cost Printed Sensors. The objective of this task was to demonstrate low cost printed sensor development by non-vacuum deposition techniques employing low thermal budget photonic curing. This work involved integration of metal and/or semiconductor layers to establish a diverse sensor technology platform. The initial application for the low cost sensor technology is a thin film temperature sensor for energy efficient buildings, defense applications, and manufacturing diagnostics.

\subsection{PROJECT BACKGROUND}

Developing roll-to-roll manufacturing processes for thin film deposition on low-cost flexible substrates will enable the fabrication of cost-effective electronics and sensor and device applications. In order to accomplish the project objectives it is necessary to demonstrate materials and coatings with low-cost nanoparticle structures manufactured using nanofermentation techniques. The development of appropriate inks is critical for high quality thin films for electronics and sensor applications. This involved evaluation of silver conductive inks by focusing on the sintering and electrical performance of ink jet printed materials. In addition, the development of non-vacuum, large-scale deposition and processing techniques for nanoparticle-based inks and pastes is important in reducing cost and energy requirements associated with processing of thin film electronics.

An important factor that impacts the eventual thin film performance is the amount of impurities or residue left behind from burn-out of surfactant materials and dispersants. It is crucial to understand not only the nature of these impurities but also the amount that can be tolerated in order to still have acceptable thin film materials for the particular application. The impact of particle size and distribution on the coating quality also need to be understood, and therefore coatings should be characterized for their structural, optical, and electrical properties. For example, resistive sensors based on nanoparticles can be used to analyze the gas sensing characteristics of $\mathrm{SnO}_{2}$ particles as a function of temperature and applied bias.

The work performed on each of task, along with the milestone demonstration goals, is summarized in the following project tasks. Project results and discussion are organized by the project task. 


\section{TASK 1: FUNCTIONALIZATION OF ZN COMPLEXES FOR OPTOELECTRONICS}

The goal of this task 1 was to develop and demonstrate low-cost, high performance phosphor materials and coatings for optoelectronic applications using zinc complexes produced by NanoFermentation (NF), deposited by low-temperature non-vacuum techniques, and sintered using Pulse Thermal Processing (PTP) technologies.

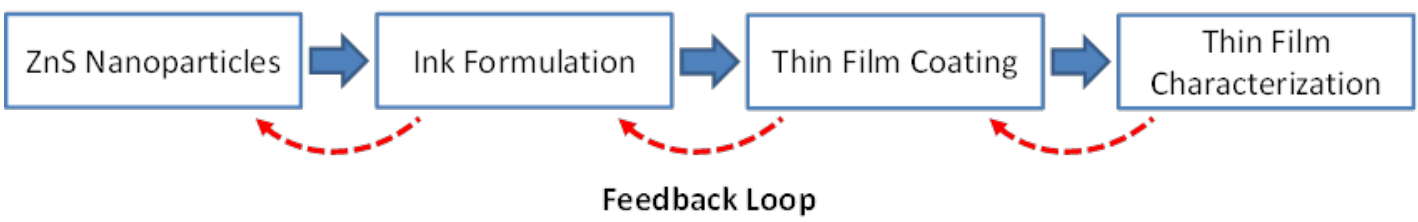

Figure 1.1. General steps in the development of NF Zn complexes for optoelectronic applications.

The overall process for the production of Zn complexes by NF and characterization of particles and thin film coatings for optoelectronic applications is summarized in Figure 1.1. Four subtasks in our development of $\mathrm{Zn}$ complexes for optoelectronic applications have been identified and are as follows:

- $\quad$ Zn complexes: The nanoparticles of $\mathrm{Zn}$ based complexes will be synthesized by a nanofermentation approach. The nanofermentation process will be optimized to achieve the target nanoparticle size and distribution suitable for high performance phosphor development. This subtask was pursued under the Low Temperature Materials Synthesis project of the Manufacturing Demonstration Facility (MDF).

- Ink development: The development of appropriate inks is critical for the realization of high quality thin films for optoelectronic applications. The ink development will bring together the elements of solvent, additives, modifiers, dispersants, and nanoparticles for the development of thin and thick films on suitable substrates. An important factor impacting the eventual thin film performance will be the amount of carbonaceous residue left behind from burn-out of surfactant materials and dispersants.

- Thin film coating: The thin films were deposited by ultrasonic spray deposition technique (Sonospray deposition). Pulse thermal processing (PTP) techniques will be explored for low thermal budget annealing of thin films. The impact of PTP treatment will be evaluated in terms of the microstructure, optical, and electrical properties of thin films.

- Thin film characterization: The thin films will be characterized in terms of the structural, optical, and electrical properties. The photoluminescence (PL) measurements will be conducted to establish the optical absorption and emission characteristics for optoelectronic applications.

In the following sections, the work performed on each of these subtasks is described in detail.

\subsection{ZnS PRODUCTION AND CHARACTERIZATION}

The details of various $\mathrm{ZnS} / \mathrm{CdS}$ nanoparticle batches that were initially investigated are highlighted in Figure 1.2. The main process variables under exploration for these batches were dosing impact, incubation time, and dopant concentration. These efforts were covered in further detail in the quarterly and final reports for the Low Temperature Materials Synthesis project of the MDF. 


\begin{tabular}{|c|c|c|c|c|c|c|}
\hline $\begin{array}{l}\text { Particle } \\
\text { Type }\end{array}$ & $\begin{array}{l}\text { Start } \\
\text { Date }\end{array}$ & Batch & $\begin{array}{l}\text { Sample } \\
\text { Number }\end{array}$ & $\begin{array}{c}\text { Sample } \\
\text { Description }\end{array}$ & Receiver & $\begin{array}{c}\text { Transfer } \\
\text { Date }\end{array}$ \\
\hline ZGT & 111107 & A & 1 & Mn (4\%) doped zinc gallate (freeze dried, $1 \mathrm{~g}$ ) & Pooran, Jay & 120221 \\
\hline ZGT & 111107 & B & 1 & $\mathrm{Cr}(1 \%)$ doped zinc gall ate (freeze dried, $1 \mathrm{~g}$ ) & $"$ & " \\
\hline ZGT & 111107 & $\mathrm{C}$ & 1 & Co $(2 \%)$ doped zinc gal late (freeze dried, $1 \mathrm{~g}$ ) & $"$ & $"$ \\
\hline \multirow[t]{7}{*}{ ZNS } & & & & Zns $5 \mathrm{mM}$ impact dosing, 10 day incubation (in DIW, $0.5 \mathrm{~g}$ ) & \multirow{7}{*}{$\begin{array}{c}\text { elemental S } \\
\text { contamination } \\
\text { : revoked }\end{array}$} & \\
\hline & & & & ZnS $0.5 \mathrm{mM} /$ day dosing, 10 day incubati on (in DIW, $0.5 \mathrm{~g}$ ) & & \\
\hline & & & & ZnS $1 \mathrm{mM}$ /day dosing, 10 day incubation (in DIW, $1 \mathrm{~g}$ ) & & \\
\hline & & & & ZnS 5 mM impact dosing, 3 day incubation (in DIW, $0.5 \mathrm{~g}$ ) & & \\
\hline & & & & ZnS 5 mM impact dosing, 5 day incubation (in DIW, $0.5 \mathrm{~g}$ ) & & \\
\hline & & & & ZnS $1 \mathrm{mM} /$ day dosing, 5 day incubation (in DIW, $0.5 \mathrm{~g}$ ) & & \\
\hline & & & & ZnS $0.5 \mathrm{mM} /$ day dosing, 5 day incubation (in DIW, $0.25 \mathrm{~g}$ ) & & \\
\hline ZNS & 120220 & A & 1 & ZnS (drop deposit on glass) & Ilia & 120306 \\
\hline ZNS & 120220 & $\mathrm{~B}$ & 1 & ZnS:Cu0.5\% (drop deposit on glass) & " & " \\
\hline ZNS & 120220 & C & 1 & ZnS Cu1\% (drop deposit on glass) & $"$ & $"$ \\
\hline ZNS & 120220 & $\mathrm{D}$ & 1 & ZnS Cu2\% (drop deposit on glass) & $"$ & 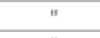 \\
\hline ZNS & 120220 & $E$ & 1 & ZnS Cu4\% (drop deposit on glass) & $"$ & $"$ \\
\hline ZNS & 120220 & $\mathrm{~F}$ & 1 & Zns Cu6\% (drop deposit on glass) & $"$ & $"$ \\
\hline ZNS & 120220 & G & 1 & ZnS Cu8\% (drop deposit on glass) & $"$ & $"$ \\
\hline ZNS & 120226 & A & 1 & ZnS $5 \mathrm{mM}$ impact dosing, 10 day incubation (in DIW, $0.5 \mathrm{~g}$ ) & Beth & 120319 \\
\hline ZNS & 120226 & B & 1 & ZnS $0.5 \mathrm{mM} /$ day dosing, 10 day incubation (in DIW, $0.5 \mathrm{~g}$ ) & \multirow{5}{*}{$\begin{array}{c}\text { elemental S } \\
\text { contamination } \\
\text { : suspend }\end{array}$} & \\
\hline ZNS & 120226 & C & 1 & ZnS $1 \mathrm{mM}$ /day dosing, 10 day incubation (in DIW, $1 \mathrm{~g}$ ) & & \\
\hline ZNS & 120226 & $\mathrm{D}$ & 1 & ZnS $5 \mathrm{mM}$ impact dosing, 5 day incubation (in DIW, $0.5 \mathrm{~g}$ ) & & \\
\hline ZNS & 120226 & $\mathrm{E}$ & 1 & ZnS 0.5 mM/day dosing, 5 day incubation (in DIW, $0.5 \mathrm{~g}$ ) & & \\
\hline ZNS & 120226 & $\mathrm{~F}$ & 1 & ZnS $0.1 \mathrm{mM} /$ day dosing, 5 day incubation(in DIW, $0.5 \mathrm{~g}$ ) & & \\
\hline ZNS & 120226 & A & 2 & ZnS $5 \mathrm{mM}$ impact dosing, 10 day (freeze dri ed, into quartz cell) & Jay & 120319 \\
\hline $\operatorname{CDS}$ & 110602 & A & 1 & CdS (110703 harvested, freeze dried, into quartz cell) & & \\
\hline
\end{tabular}

Figure 1.2. Various NF nanoparticle batches investigated.
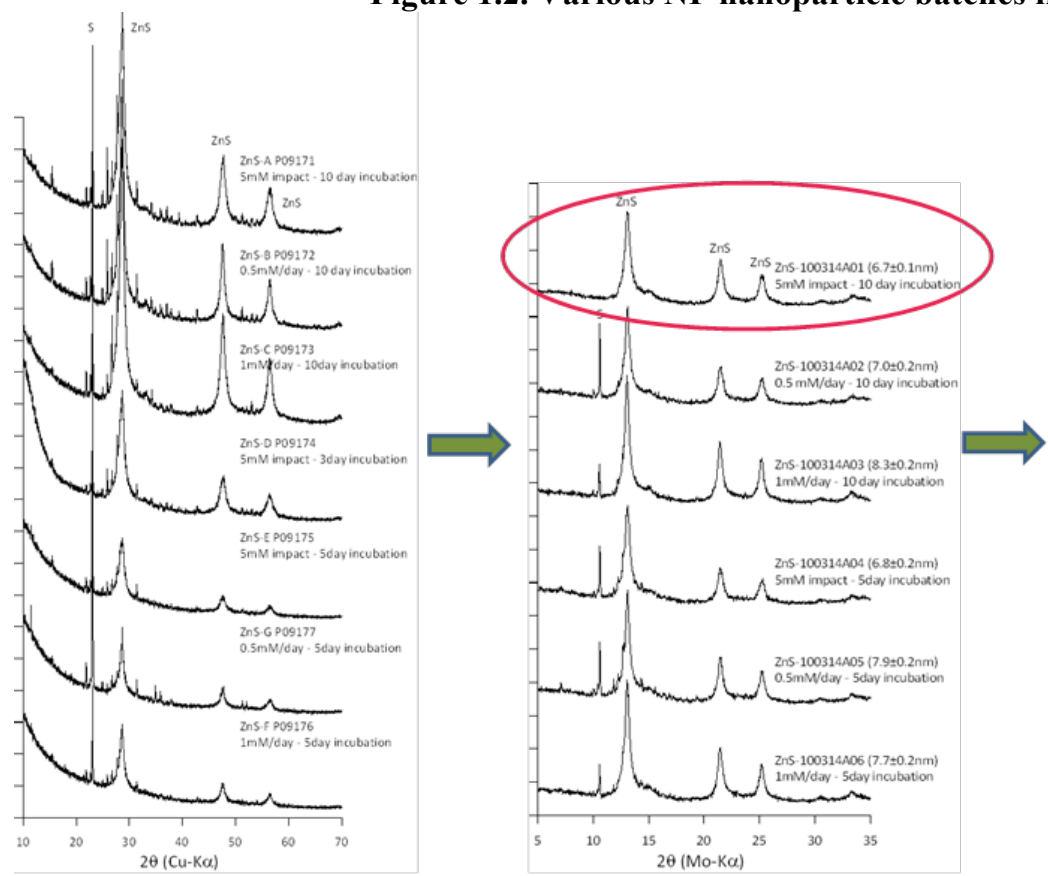

On-going

Found new condition

- $5 \mathrm{mM}$ impact

- Thiosulfate $6 \mathrm{mM}$

- No sonication

- Two kinds of medium

- Two incubation time

of 5 and 10 days

Figure 1.3. The impact of incubation time on nanoparticle growth and microstructure.

X-ray Diffraction (XRD) was used to optimize the NF process for Zn complex synthesis. Figure 1.3 shows the effect of incubation time on the microstructure of $\mathrm{ZnS}$ nanoparticles. As shown in Figure 1.3, the optimum process conditions result in well-defined crystalline $\mathrm{ZnS}$ phase. The bio-ZnS was produced by the incubation of $10 \mathrm{mM}$ of glucose as electron donor, $10 \mathrm{mM}$ of thiosulfate as electron acceptor and sulfur source, and $2 \%(\mathrm{v} / \mathrm{v})$ mid-log growth TOR-39 at $65^{\circ} \mathrm{C}$. Zinc salt was dosed as a single dose injecting a total target amount of $\mathrm{Zn}$ salt or a discrete pulsed dose injecting a partial amount at a time with multiple times until approaching total target amount. The first batch was revoked due to co-existing of elemental sulfur $\left(\mathrm{S}^{0}\right)$ (Figure 1.2). The protocol we used had never produced elemental sulfur previously, and the reason for its production in this batch is not certain. We 
then we reduced thiosulfate concentration to $8 \mathrm{mM}$. The impact of reduced concentration on the crystallinity and sulfur incorporation is shown in Figure 1.3. Elemental sulfur was detected for both single and discrete pulsed dosing at second batch (ZNS120226 series). But average crystallite size obtained from the X-ray diffraction patterns explicated that single dosing induced smaller size compared to discrete pulsed one. The batch circled in red gave the purest $\mathrm{ZnS}$ phase with minimal elemental sulfur.

Based on the observed x-ray diffraction results (Figure 1.3), we proceeded to make a third batch with a reduced thiosulfate concentration of $6 \mathrm{mM}$ and a single dosing method. Additionally we tried a different medium composition (Figure 1.4). We found that a single dose with 10 day incubation in Bmedium produced smaller size and better crystallinity $\mathrm{ZnS}$ nanoparticles (results circled in red in Figure 1.4).

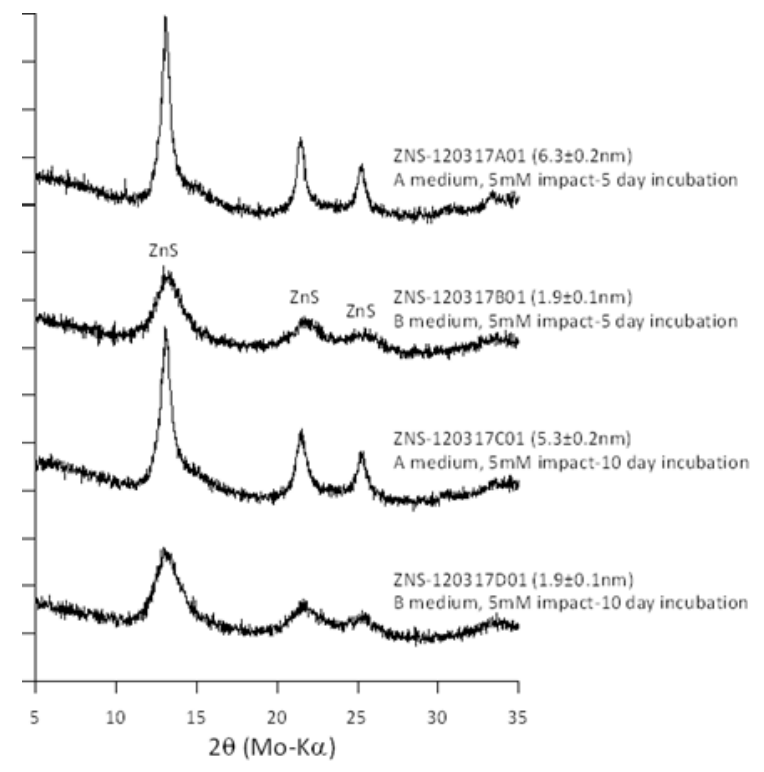

Figure 1.4. The impact of single dosing, thiosulfate concentration, and increased incubation time on $\mathrm{ZnS}$ nanoparticle growth and crystallinity.

\subsubsection{ZnS Process Scale-up}

Based on the understanding of phase formation kinetics, we scaled up to a $4 \mathrm{~L}$ batch composed of simultaneous 4 batches of 1L medium scale (ZNS120403) and a 24L batch composed of dual parallel reactors of 12L (ZNS120426) (Figure 1.5(a)). This result showed that scaled-up nanofermentation can be advantageous in shortening the process time and saving energy. For the same synthesis condition, the $24 \mathrm{~L}$ batch showed higher crystallinity (Figure $1.5(\mathrm{~b})$ ) than the $4 \mathrm{~L}$ batch, indicating that the incubation period can be reduced without sacrificing material quality. 

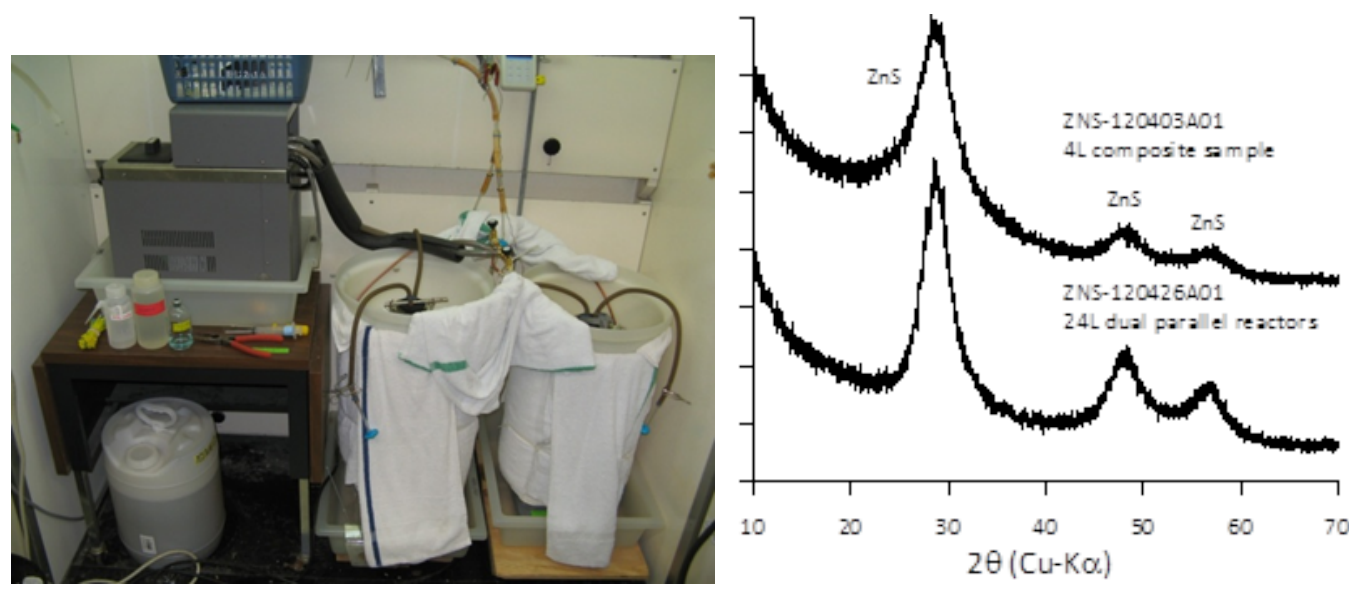

Figure 1.5. (a) Setup for process scale up, and (b) $x$-ray diffraction patterns indicating that phase formation kinetics are well-maintained for the scaled up process.

\subsubsection{ZnS Photoluminescence Response}

Photoluminescence (PL) measurements were conducted to gain further insight into the $\mathrm{ZnS}$ material characteristics. As shown in Figure 1.6(a), a short incubation (5 day) single dose sample exhibited the strongest PL emission. Based on the PL response, the incubation period was shortened to 5 days for the $2^{\text {nd }} 24 \mathrm{~L}$ ZNS120515 batch. The crystallinity of the ZnS nanoparticles was not influenced by the shorter incubation time as shown in Figure 1.6(b). The PL measurements are limited by the setup capabilities. The photon energy from our illumination light source is too small to see the fundamental excitonic PL from the $\mathrm{ZnS}$ nanocrystals. We used an illumination wavelength of $458(2.71 \mathrm{eV})$, while an illumination wavelength $>3.9 \mathrm{eV}(<317 \mathrm{~nm})$ is required to see the fundamental PL spectra.
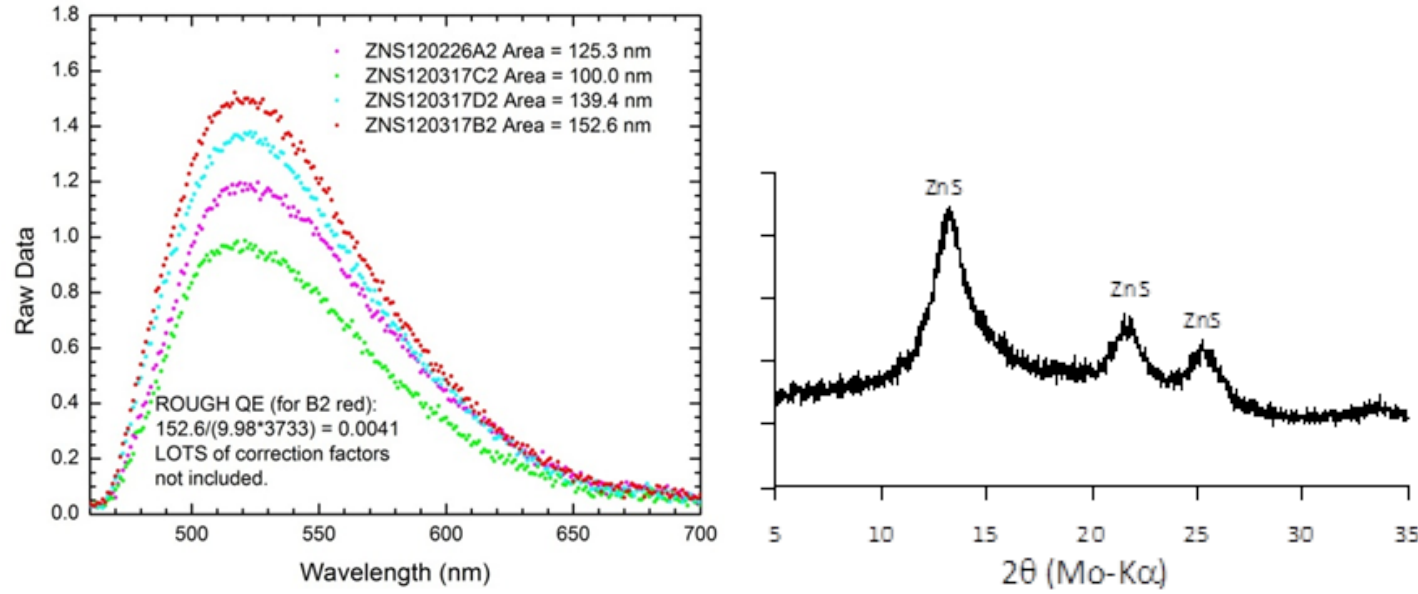

Figure 1.6. The impact of shorter incubation time on the (a) PL response for the batches shown in the figure, and (b) crystallinity of ZnS nanoparticles for batch number ZNS120515. 

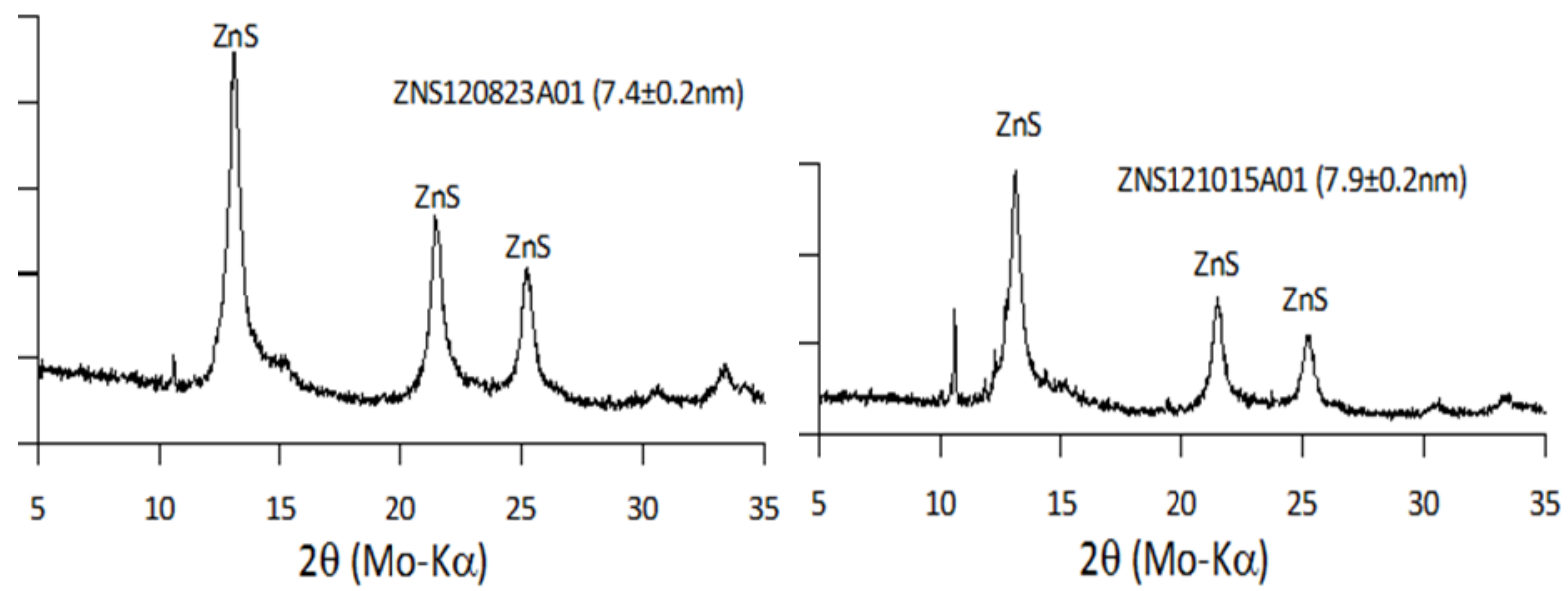

Figure 1.7. X-ray diffraction patterns of $\mathrm{ZnS}$ nanoparticles with estimated particle size of (A) $7.4 \pm 0.2$ $\mathrm{nm}$ and (B) $7.9 \pm 0.2 \mathrm{~nm}$.

In the work described above, the size of NF-produced $\mathrm{ZnS}$ nanoparticles was in the range of 2-3 nm (as measured by XRD). Subsequently, larger sized particle sizes (sample ZNS120823A01) were produced with estimated particle sizes in excess of $7 \mathrm{~nm}$ (see Figure 1.7) in quantities sufficient for PL and dispersion analyses. Sample ZNS121015A01 resulted in similar sized ZnS particles, in scaling up to $24 \mathrm{~L}$ batch fabrication, even though that procedure had produced $>12 \mathrm{~nm}$ sized $\mathrm{ZnS}$ particles when fabricated in a $50 \mathrm{ml}$ batch process.

\subsubsection{Nanoparticulate Solution Purification}

Techniques were also explored for decreasing the organic content of the nanoparticulate solutions. Since this is a biologically-based process, it is necessary to provide nutrients for the bacteria to survive and produce the nanoparticles. During the course of this work, we postulated that the organic materials used during the nanoparticle synthesis technique were responsible for potential carbonaceous residue witnessed after thin film sintering. Therefore, various nutrient sources were investigated to reduce the organic content of the nanoparticle solutions. Table 1.1 lists a number of the nutrient materials considered and Figure 1.8 shows the effect on the optical density (turbidity) of the various samples. Three of the most promising samples (i.e. glucose, xylose, and pyruvate) were analyzed by XRD (Figure 1.9), and ZnS nanoparticle formation was confirmed for two of the samples (glucose and xylose).

Table 1.1. Nutrients evaluated for NanoFermentation of ZnS to reduce organic content

\begin{tabular}{|l|l|}
\hline Nutrients & Formula and molecular weight \\
\hline Glucose (standard) & C6H12O6, MW $=180.16$ \\
\hline Xylose & C5H10O5, MW $=150.13$ \\
\hline Lactate & C3H6O3, MW $=90.08$ \\
\hline Pyruvate & C3H4O3 MW $=88.06$ \\
\hline Acetate & C2H3O2, MW $=60.05$ \\
\hline Formate & HCOO, MW $=45.02$ \\
\hline
\end{tabular}




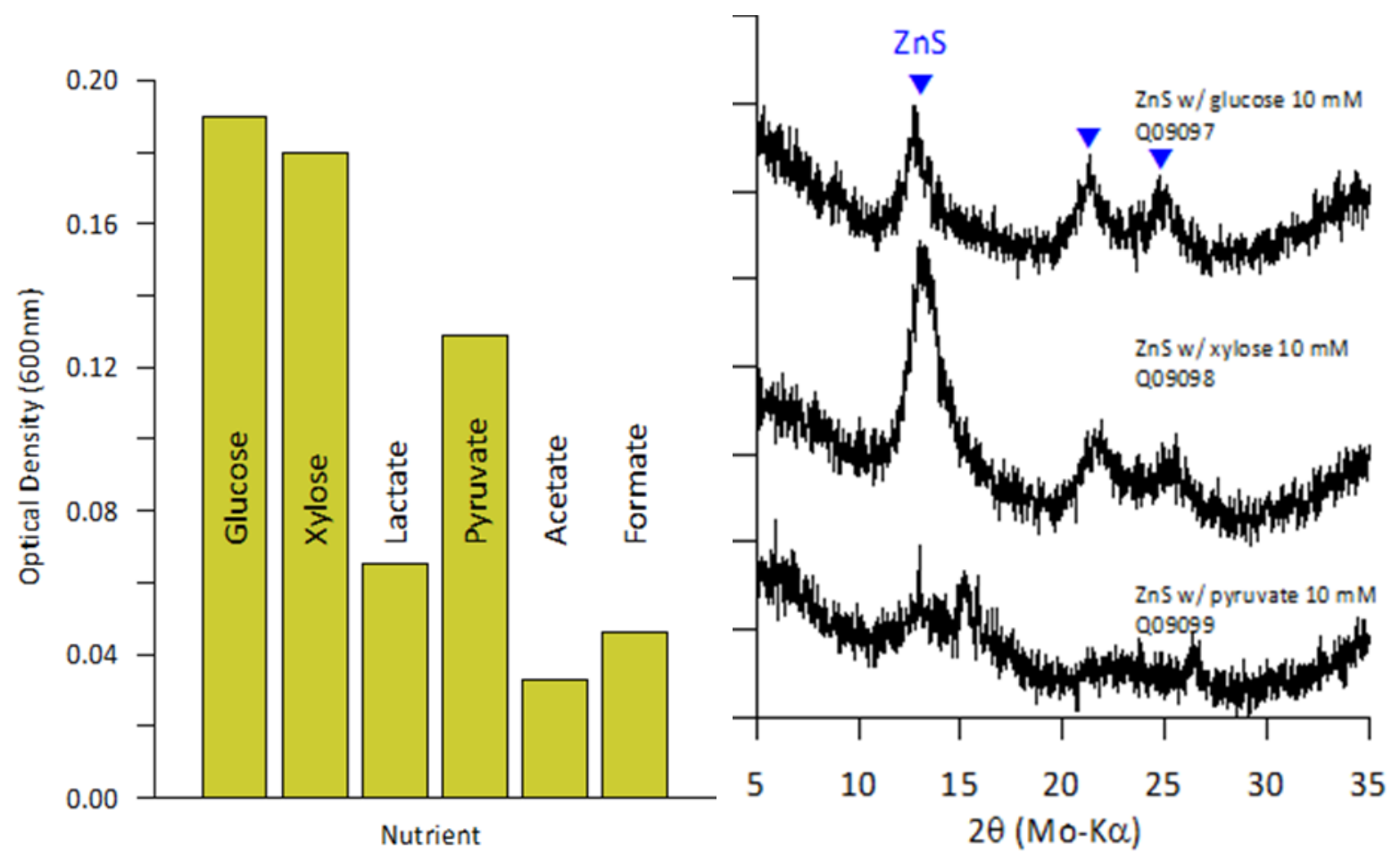

Figure 1.8. Optical density measurement of $\mathrm{ZnS}$ nanoparticle solutions synthesized with various nutrient sources.

Figure 1.9. X-ray diffraction pattern of $\mathrm{ZnS}$ nanoparticles produced with glucose (top), xylose (middle), and pyruvate (bottom

In order to further reduce the organic content of the nanoparticle solutions, the abiotic production of $\mathrm{ZnS}$ nanoparticles was also explored. Instead of using bacteria, chemical and electrochemical routes were explored to provide nucleation sites and reduction potential. We demonstrated that it was possible to produce $\mathrm{ZnS}$ nanoparticles in degased water with the addition of Cysteine-S under careful $\mathrm{pH}$ control. These techniques were described in additional detail in the Low Temperature Synthesis FY2012 Q4 MDF quarterly report. The abiotic production process provided nanoparticles with increased particle sizes of up to $9.5 \mathrm{~nm}$ as estimated by XRD (Figure 1.10).

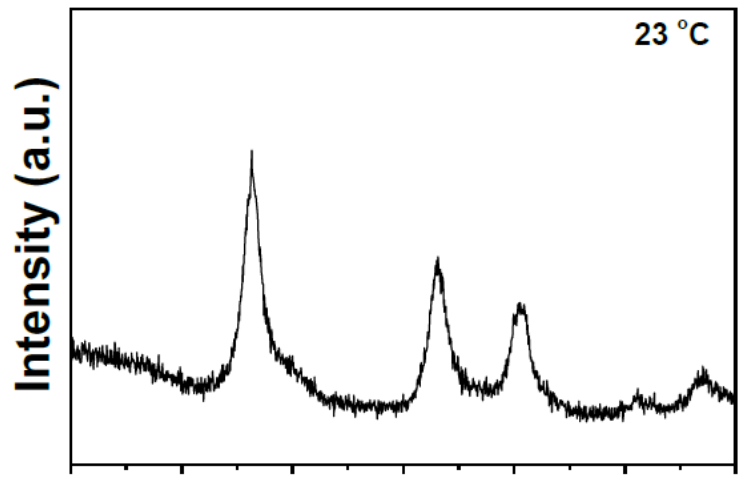

$2 \theta$ (Degree) [Mo-k $\alpha]$

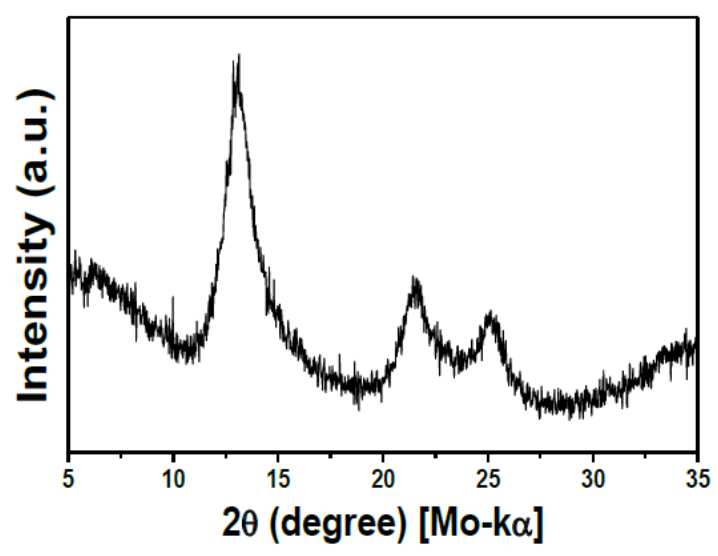

Figure 1.10. X-ray diffraction patterns of $\mathrm{ZnS}$ nanoparticles produced (A) abiotically and (B) biotically, demonstrating an increase in particle size from 4.6 to $9.5 \mathrm{~nm}$. 
The abiotic synthesis of $\mathrm{ZnS}$ nanoparticles also demonstrated a marked decrease in the organic content, as demonstrated by reduced organic bonding (circled portion of FTIR spectrum in Figure $1.11)$

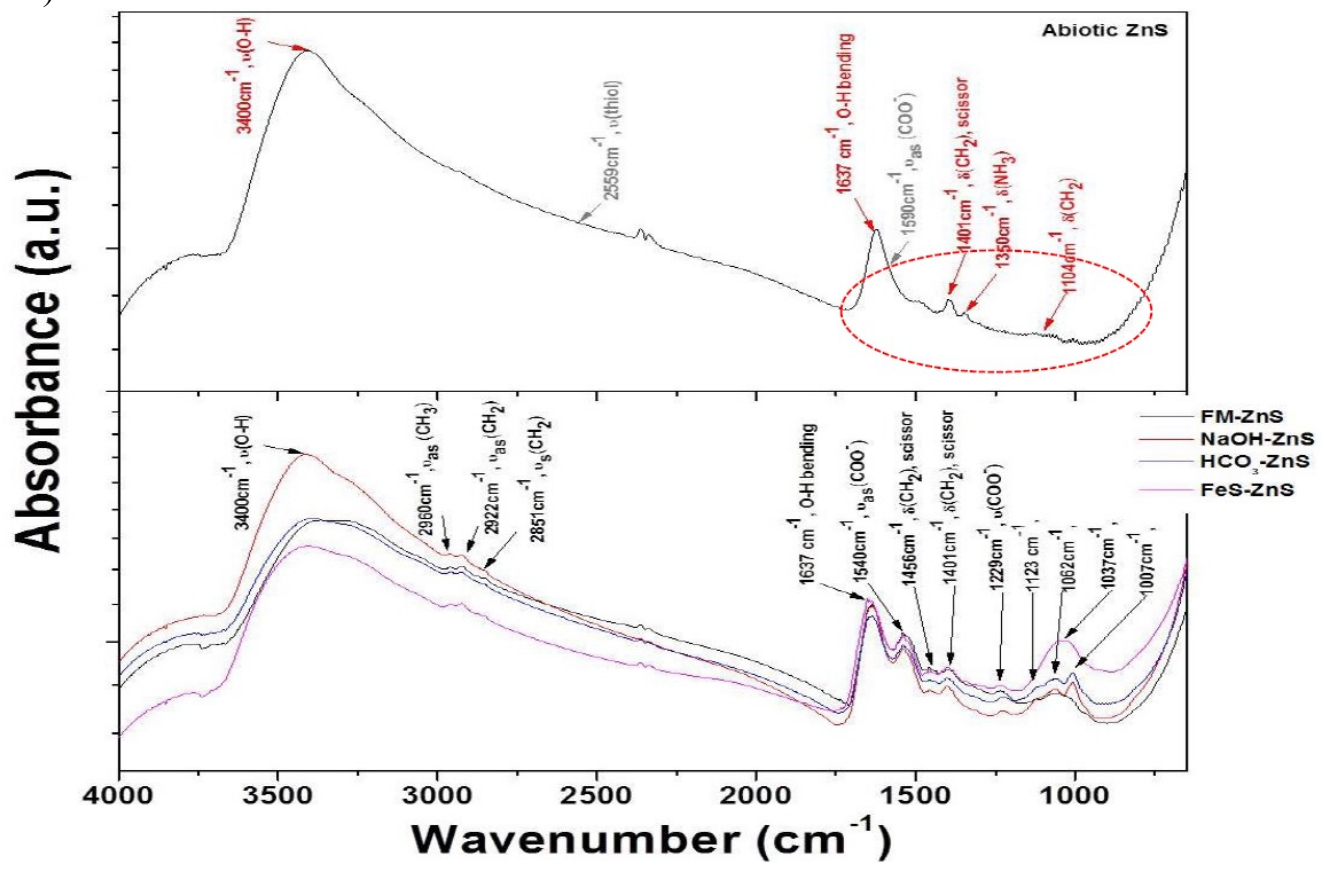

Figure 1.11. Fourier transfer infrared (FTIR) spectroscopy of abiotic (top) versus biotic (bottom) production of $\mathrm{ZnS}$, showing reduced organic bonding for abiotic production.

Initial attempts to fabricate the NP ZnS resulted in agglomerates that were too large. Subsequent studies were performed to further disperse the $\mathrm{ZnS}$ nanoparticles in deionized water. Variables of interest during post recovery treatment included surfactant types, surfactant concentration, $\mathrm{ZnS}$ dispersed content, sonication method and time, and sedimentation periods. Currently, the best results have been achieved with the dispersant NTA, producing aggregates with average size $\sim 120 \mathrm{~nm}$ (Figure 1.12).

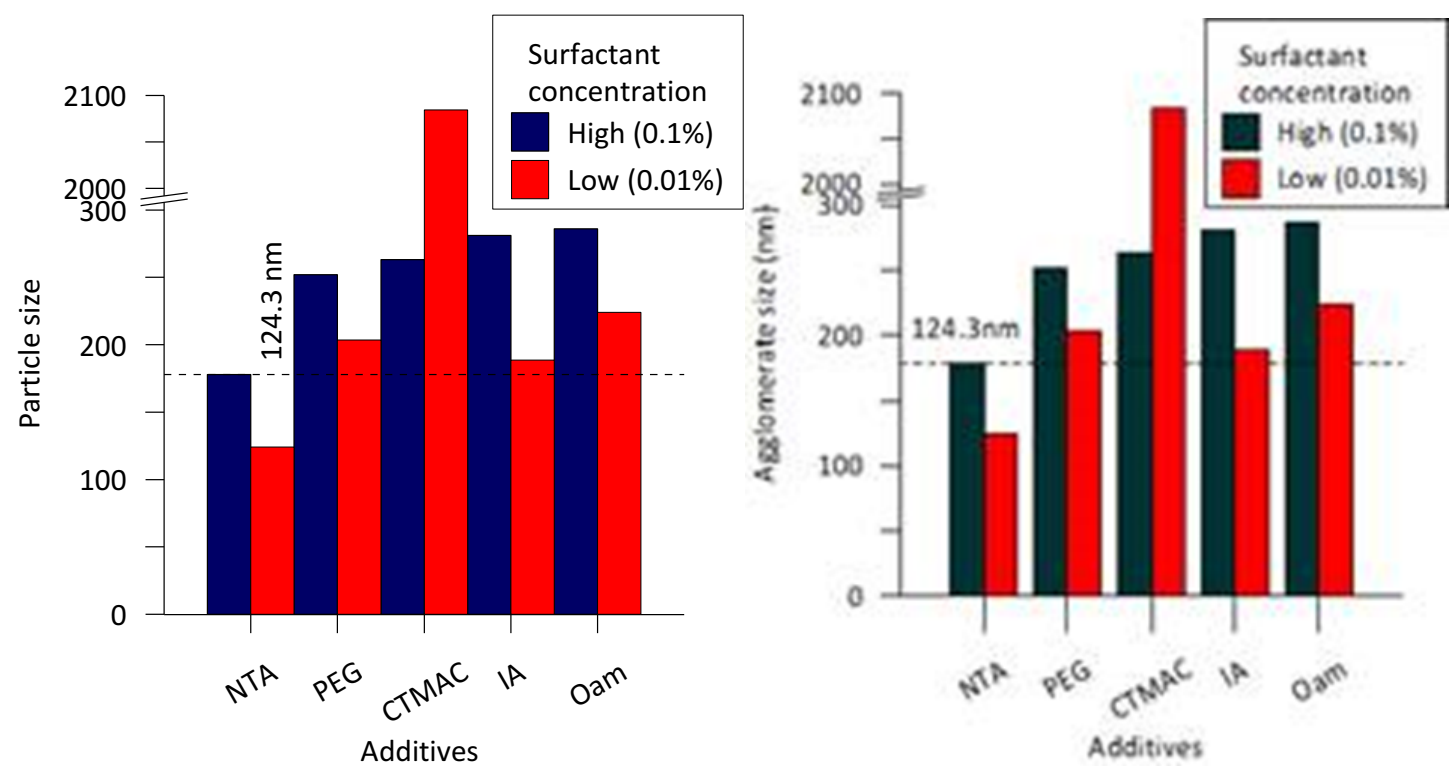

Figure 1.12. $\mathrm{ZnS}$ average agglomerate size after post recovery treatment with the various surfactants. 
We were unable to reproduce the impressive results from earlier work (down to $25 \mathrm{~nm}$ ), so it appears that data point may have been a measurement artifact. We also investigated ways to accomplish insitu dispersion of the nanoparticles. Use of the dispersant thioglycerol gave almost the same aggregate size to NTA, however all dispersant candidates included in the post recovery treatment experiments gave large average crystallite sizes. Surfactant addition timing was also tested as some surfactants block microbial activity and can be used as another carbon source for the bacteria. The average sizes of the $\mathrm{ZnS}$ nanoparticles obtained in these studies are summarized in Figure 1.13, and show that the time of the addition of the thioglycerol made little difference on the size of the resultant $\mathrm{ZnS}$ particles. A second study looked at the effect of the amount and timing of the thioglycerol on the size of the $\mathrm{ZnS}$ agglomerates. The results shown in Figure 1.14 indicate again that timing is relatively unimportant, but there is a small decrease in $\mathrm{ZnS}$ agglomerate size with increasing thioglycerol concentration. 

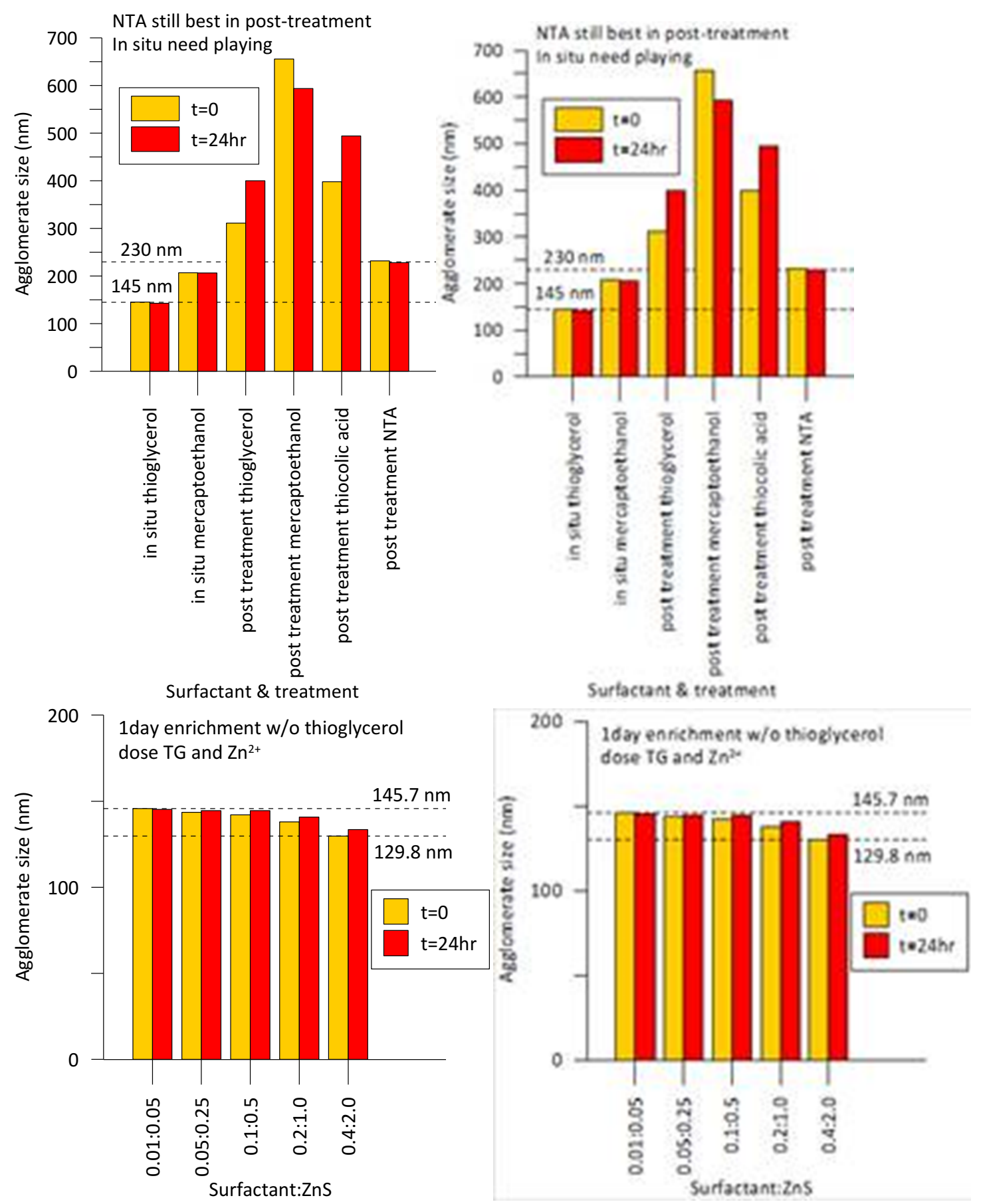

Figure 1.13. (right) $\mathrm{ZnS}$ average agglomerate size for various in-situ versus post recovery treatments.

Figure 1.14. ZnS agglomerate size Comparison study for in-situ treatment according to dosing.

The measured $\sim 120 \mathrm{~nm}$ aggregate size of biologically produced $\mathrm{ZnS}$ nanoparticles is supported by the other study reported in the literature (Moeau JW, Weber PK, Martin MC, Gilbert B, Hutcheon ID, Banfield JF. "Extracellular proteins limit the dispersal of biogenic nanoparticles." Science 2007; 316: 1600 ) that showed that aggregation of $3 \mathrm{~nm}$ inorganically produced $\mathrm{ZnS}$ particles, capped with an amino acid, initially occurred rapidly to form $100 \mathrm{~nm}$ diameter aggregates. The growth rate of the aggregates subsequently slowed greatly or ceased after one week. A minimum aggregate size of 
around $100 \mathrm{~nm}$ from many dispersion cases, and several of these aggregates were examined by surface characterization.

All samples were studied using FT-IR, regardless of the medium (mostly buffer) used to disperse the $\mathrm{ZnS}$ particles. These studies clearly showed that the samples contained organic matter as indicated by $v\left(\mathrm{CH}_{2}\right)$ and $v\left(\mathrm{CH}_{3}\right)$ groups at $2960 \mathrm{~cm}^{-1}, 2922 \mathrm{~cm}^{-1}$, and $2851 \mathrm{~cm}^{-1}$, and scissor modes of $\mathrm{CH}_{2}$ at 1456 $\mathrm{cm}^{-1}$ and $1401 \mathrm{~cm}^{-1}$ (Figure 1.15a). The spectra from all the samples seemed to be related to $-\mathrm{COO}^{-}$ groups, as featured by $v(\mathrm{COO})$ at $1540 \mathrm{~cm}^{-1}$ and $1229 \mathrm{~cm}^{-1}$. XPS spectrum (Figure 1.15b) also clearly exhibited the dominant hydroxyl carbon $(\mathrm{C}-\mathrm{O}, 286.2 \mathrm{eV})$ and carbonyl carbon $(\mathrm{C}=\mathrm{O}, 287.5 \mathrm{eV})$ peaks. Another spectrum (Figure 1.15c) exhibited a prominent peak at $\sim 399.5 \mathrm{eV}$, that most likely belonged to amine $\left(-\mathrm{NH}_{2}\right)$ and a smaller peak at $\sim 402.1 \mathrm{eV}$ which could be a protonated form of the amine. These observations supports the idea that the final end products of fermentation included lactate and acetate as well as microbially derived extracellular protein, including amine and carboxylic acids, which most likely facilitated, not only keeping the average nano sized dimensions of the crystallite $\mathrm{ZnS}$ nanoparticle, but also increased the particle aggregation.
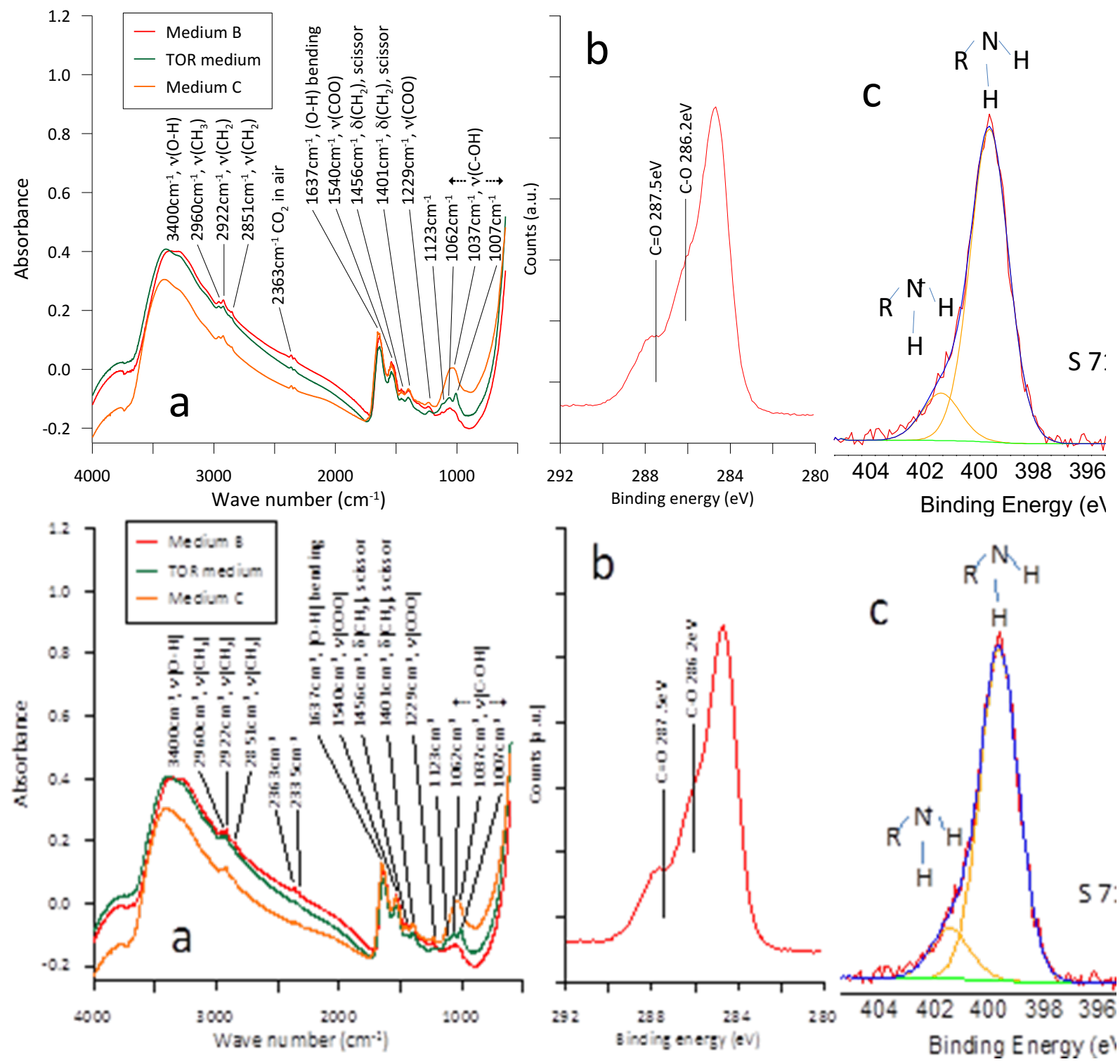
Figure 1.15. FT-IR spectroscopy and XPS measurement of the surface of NP ZnS formed by Thermoanaerobacter X513. (a): FT-IR spectra of the NP ZnS using various buffer systems; (b), XPS spectrum showing $\mathrm{C}-\mathrm{O}$ and $\mathrm{C}=\mathrm{O}$ bonding; (c), XPS spectrum showing amine $\left(-\mathrm{NH}_{2}\right)$ and protonated amine bonding

\subsubsection{Zn Gallate Manufacturing and Characterization}

The primary effort within this subtask is focused on the production of colored phosphor nanoparticles by doping different metals into the zinc gallate host structure, as well as evaluating their optical properties as a function of the various post fabrication treatments. The initial work in this task is focused on the fabrication of various colored phosphors, which could produce red, green, and blue (RGB) emissions using small $10 \mathrm{~mL}$ reactor vessels. This effort was subsequently scaled up from 10 $\mathrm{mL}$ to $2 \mathrm{~L}$ culture bottles $-\mathrm{a} 200 \mathrm{x}$ increase in scale. The next step was to scale production of the phosphors using several $12 \mathrm{~L}$ carboy reactor vessels, which represented a $2,400 x$ scale-up from the initial $10 \mathrm{~mL}$ test tube production capabilities. The reactor vessels used in each of these scale-up experiments are shown in Figure 1.16. After one week of incubation, the phosphors were washed in deionized water and freeze dried.

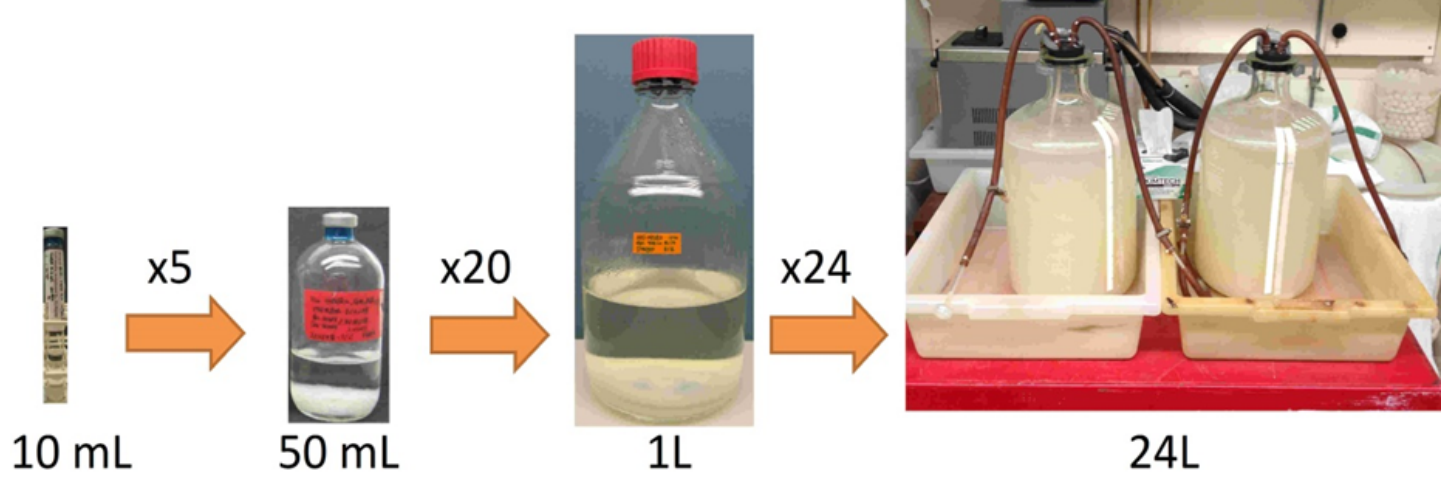

Figure 1.16. Scale up of NanoFermented phosphor production with scale factor of 100.

Table 1.2. Experiments performed to prepare the wet and dried nanoparticle phosphors

\begin{tabular}{|l|l|}
\hline Sample & Description \\
\hline ZGT120529A01 & $\begin{array}{l}\text { Using precursor with Co } 1 \% \text { replacing Zn, freeze dried } 1.686 \mathrm{~g} / 3 \mathrm{~L} \\
\text { for BLUE }\end{array}$ \\
\hline ZGT120529B01 & $\begin{array}{l}\text { Using precursor with Mn } 4 \% \text { replacing Ga, freeze dried } 2.015 \mathrm{~g} / 3 \mathrm{~L} \\
\text { for GREEN }\end{array}$ \\
\hline ZGT120529C01 & $\begin{array}{l}\text { Using precursor with Cr } 1 \% \text { replacing Ga, freeze dried } 1.875 \mathrm{~g} / 3 \mathrm{~L} \\
\text { for RED }\end{array}$ \\
\hline ZGT120711A01 & $\begin{array}{l}\text { Using precursor with Mn 4\% replacing Zn, freeze dried } 1.574 \mathrm{~g} / 2 \mathrm{~L} \\
\text { for GREEN }\end{array}$ \\
\hline ZGT120711B01 & $\begin{array}{l}\text { Using precursor with Mn } 4 \% \text { replacing Zn with Gallium Nitrate } \\
\text { Hydrate, freeze dried }(0.897 \mathrm{~g} / \mathrm{L}) \text { for GREEN }\end{array}$ \\
\hline ZGT120614A01 & Using precursor Co $1 \%$ replacing Zn, 6.7\% solution in water \\
\hline ZGT120614B01 & Using precursor Mn 4\% replacing Ga, 5.6\% solution in water \\
\hline ZGT120614C01 & Using precursor Cr $1 \%$ replacing Ga, 6.3\% solution in water \\
\hline ZGT120626A01 & Using precursor Mn 4\% replacing Zn, 7.9\% solution in water \\
\hline &
\end{tabular}

In the initial small-scale experiments, Co-doped zinc gallates produced a blue emission, Mn-doped zinc gallates (where the $\mathrm{Mn}$ replaced either the $\mathrm{Zn}$ or $\mathrm{Ga}$ in nominal mole fraction in precursor composition) produced a green emission, and $\mathrm{Cr}$ and Eu doped zinc gallates (where the dopant replaced the $\mathrm{Ga}$ ) produced a red emission. Simple blending of Co for blue, $\mathrm{Mn}$ for green, and $\mathrm{Cr}$ for 
red resulted in doped zinc gallates that produced white light.

In follow-on experiments, we focused on Co replacing $\mathrm{Zn}$ for blue, Mn replacing $\mathrm{Zn}$ rather than Ga (due to yield), and $\mathrm{Cr}$ rather than $\mathrm{Eu}$ (due to higher price of rare earth elements) replacing $\mathrm{Ga}$ precursors and their phosphor production. A FeS medium, which was used for ZnS synthesis in the first year of the project, was incubated with $10 \mathrm{mM}$ of glucose and $2 \%$ mid-log growth thermophilic bacteria (Tsp). After $24 \mathrm{hr}$, the $\mathrm{Zn}$ with a $2.5 \mathrm{mM}$ dopant and $\mathrm{Ga}$ with a $5 \mathrm{mM}$ dopant with the same volume of 1.5M MOPS buffer were dosed, and then incubated for one week. Table 1.2 summarizes the experiments that were performed to fabricate the RGB phosphors.

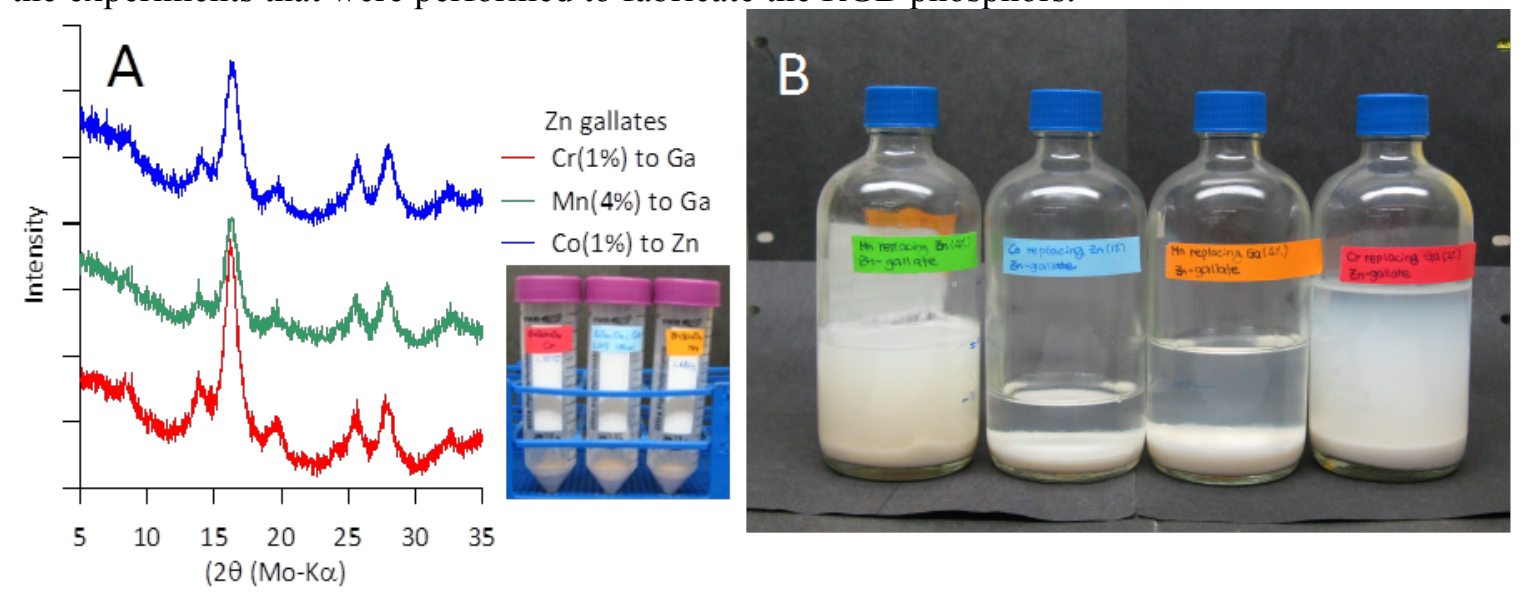

Figure 1.17. XRD patterns from representative RGB Zn-gallate phosphor before post-treatment.

Gallium nitrate was also used in some experiments as the precursor chemical for the formation of $\mathrm{Zn}$ gallate instead of the more conventional gallium chloride. Some representative samples were analyzed and the XRD spectra shown in Figure 1.17. The finding that metal-doped zinc gallate formation was possible using the adjusted $\mathrm{pH}$-Eh micro-environment mediated by microbial activity, without reducible metal or non-metal components in the system, was a unique approach to phosphor production. Consequently, ORNL filed this as US Patent Application Number 61/644,211 (2012) on May 8, 2012 and filed this as international patent application (PCT) on March 14, 2013.

Table 1.3. Agglomerate Size Results for the Zinc Gallate Study

\begin{tabular}{|c|c|c|c|c|c|c|}
\hline & & \multicolumn{4}{|c|}{ Sizes $(\mu \mathrm{m})$} & Sample \\
\hline Modification & Sonicated & & Diameter on Cumulative \% & ID Number \\
\hline & & Mean & $10 \%$ & $50 \%$ & $90 \%$ & \\
\hline Co replacing $\mathrm{Zn} \mathrm{(1 \% )}$ & & 6.77012 & 3.2581 & 6.3241 & 10.8869 & ZGT120614A01 \\
\hline Co replacing Zn (1\%) & Yes & 4.69867 & 1.8551 & 4.3382 & 7.9792 & ZGT120614A01 \\
\hline Mn replacing Ga (4\%) & & 8.70829 & 4.7768 & 8.1905 & 13.2274 & ZGT120614B01 \\
\hline Mn replacing Ga (4\%) & Yes & 6.39268 & 3.1200 & 6.0089 & 10.1463 & ZGT120614B01 \\
\hline Cr replacing Ga (1\%) & & 7.26818 & 3.5818 & 6.8082 & 11.5189 & ZGT120614C01 \\
\hline Cr replacing Ga (1\%) & Yes & 5.44319 & 2.2125 & 5.0333 & 9.1934 & ZGT120614C01 \\
\hline Mn replacing Zn (4\%) & & 7.21283 & 3.5240 & 6.7367 & 11.4930 & ZGT120626A01 \\
\hline Mn replacing Zn (4\%) & Yes & 4.95120 & 1.7619 & 4.4794 & 8.7174 & ZGT120626A01 \\
\hline
\end{tabular}

The experiments to produce NF zinc materials produced particles that were highly agglomerated with particle sizes in the 10 micron size range. A significant effort was pursued to reduce the size of the agglomerated NF zinc materials using sonication techniques. These experiments and the analyzed particle sizes are summarized in Table 1.3. 
Additional efforts were focused on integrating dispersant agents into the Nanofermentation synthesis process in order to reduce agglomeration and limit organic contamination. A detailed description of these efforts can be found in the Q1 FY2013 Low Temperature Materials Synthesis project of the MDF quarterly report.

Each of the RGB color phosphors was produced under identical process conditions in a $24 \mathrm{~L}$ growth medium (Figure 1.16). All samples resulted in the similar average crystallite size at around 2-3 nm calculated by X-ray diffraction patterns (Figure 1.18). Also, the crystallinity of each RGB color phosphor was found to be inversely proportional to the ionic toxicity of the doping metals. The low toxicity of doped manganese compared to cobalt and chromium exhibited the highest crystallinity, even though doping amount was high at $4 \%$ compared to $1 \%$ cobalt and $1 \%$ chromium.

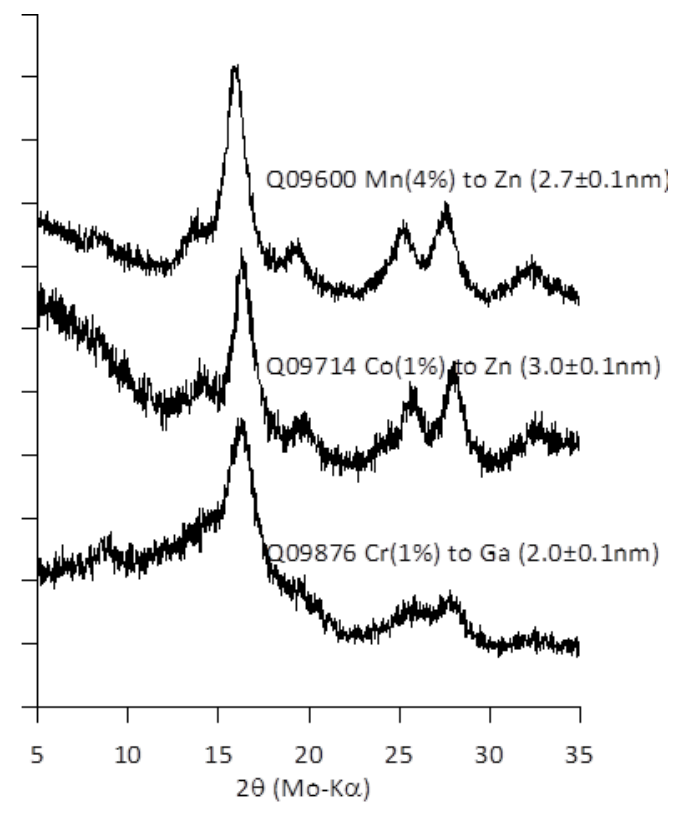

Figure 1.18. X-ray diffraction patterns for each of the RGB color phosphors produced in the 24L reactor.

Table 1.4 Stock solution compositions

\begin{tabular}{rccc}
\hline Final Input $(\mathrm{M})$ & $\mathrm{Mn}(\mathrm{M})$ & $\mathrm{Ga}(\mathrm{M})$ & Total $(\mathrm{M})$ \\
$\mathrm{Zn}(\mathrm{M})$ & 0.008 & 0.556 & 0.75 \\
0.187 & 0.009 & 0.536 & 0.75 \\
0.206 & 0.009 & 0.517 & 0.75 \\
0.223 & 0.010 & 0.500 & 0.75 \\
0.240 & 0.011 & 0.484 & 0.75 \\
0.255 & 0.011 & 0.469 & 0.75 \\
0.270 & 0.012 & 0.455 & 0.75 \\
0.284 & & & \\
\hline
\end{tabular}

Earlier work on the project found that the photoluminescence of zinc gallate samples showed some variance in that starting the incubation with all ingredients and bacteria at initial and shorter incubation periods resulted in better photoluminescence. Another variable is the stoichiometry of the precursor solution to enhance photoluminescence. Previous elemental analysis of final product using a $\mathrm{Zn}: \mathrm{Ga}=1: 2$ stock solution was found to be very close to $1: 2$. Therefore we tuned $30 \%$ zinc variation with $10 \%$ increment from $\mathrm{Zn}$ :Ga ratio $\left(\mathrm{Zn}_{1 \pm 0.3}: \mathrm{Ga}_{2}\right)$. The goal was to achieve $\mathrm{Mn} 4 \%$ doped 
zinc gallate, which exhibited the highest green emission. Stock solutions were prepared at $4 \% \mathrm{Mn}-$ doping (see Table 1.4) and incubated with $10 \mathrm{mM}$ glucose, $2 \%$ mid-log growth bacteria stock solution, total $7.5 \mathrm{mM}$ stock solution, and additional MOPS buffer to keep the $\mathrm{pH}$ between 7.5-8.0. Figure 1.19 show samples of nanofermented zinc gallate precipitates.

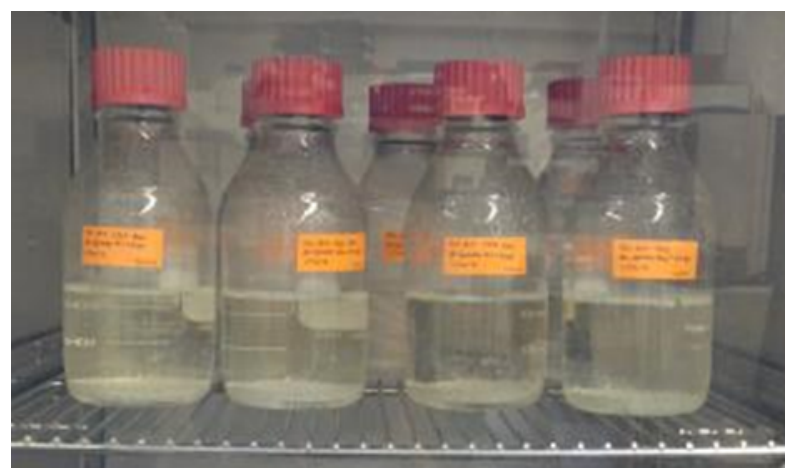

Figure 1.19. NanoFermented zinc gallate precipitates.

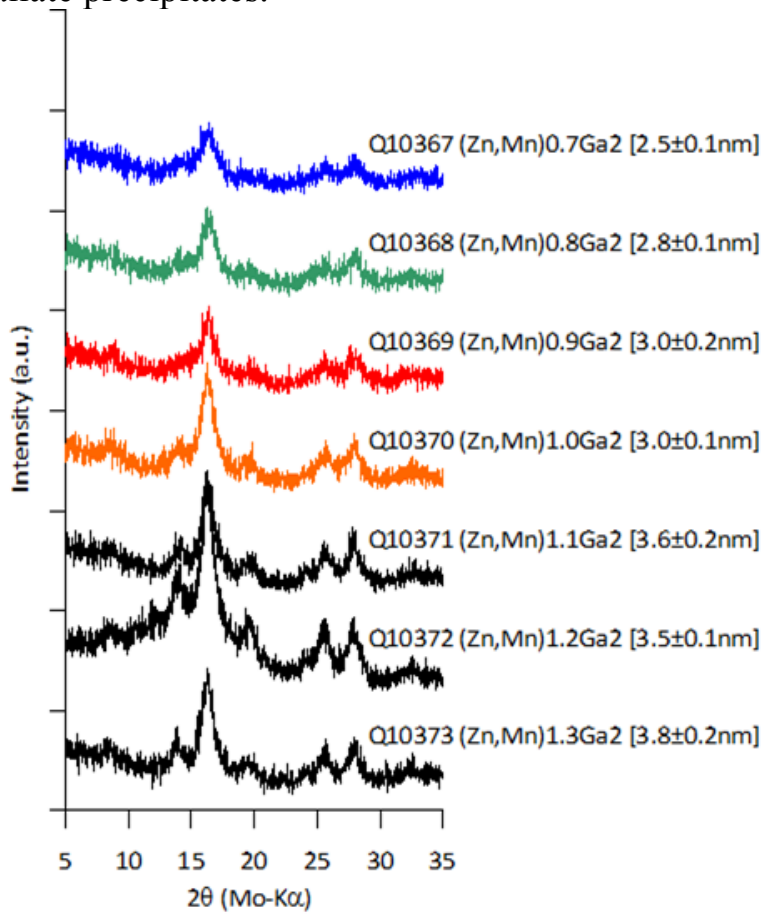

Figure 1.20. X-ray diffraction patterns of zinc gallates using various $([\mathrm{Zn}]+[\mathrm{Mn}]) /[\mathrm{Ga}]$ ratios.

\subsubsection{Zn, Sn, Ni, Co and Cu Sulfide Manufacturing}

Four additional metal sulfides were studied; namely $\mathrm{SnS}, \mathrm{Ni}_{3} \mathrm{~S}_{4}, \mathrm{CoS}_{2}$, and $\mathrm{CuS}$. These nanoparticles were produced using a 24 liter scale production capability. In order to achieve the 24 liter reaction scale, 12 liters of growth medium was added to two 13.25 liter glass carboys. The carboys were equipped with ventilation ports in the headspace and were autoclaved for a period of 2 hours. The carboys were subsequently cooled to $70^{\circ} \mathrm{C}$ in the autoclave. The samples were further cooled with continuous purging using $\mathrm{N}_{2}$ gas through a 0.2 -micron filter overnight and put into plastic containers, which were connected to a $65^{\circ} \mathrm{C}$ water bath. Figure 1.21 shows examples of samples of metal sulfides ( $\mathrm{SnS}, \mathrm{Ni3S} 4, \mathrm{CoS} 2$, and $\mathrm{CuS})$ produced in the 24 liter reactor, which will be used to fabricate our thin films metal oxide gas sensors.
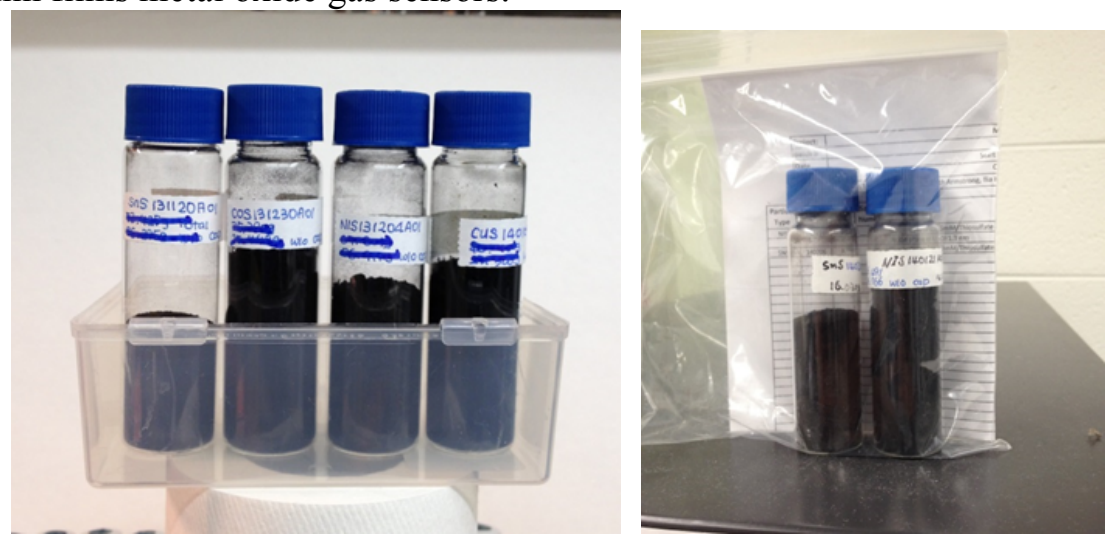
Figure 1.21. (a) Examples of samples of metal sulfides ( $\mathrm{SnS}, \mathrm{Ni}_{3} \mathrm{~S}_{4}, \mathrm{CoS}_{2}$, and $\mathrm{CuS}$ ) produced in our 24 liter reactor. These will be used to fabricate thin films metal oxide gas sensors. (b) $\mathrm{SnS}$ and $\mathrm{Ni}_{3} \mathrm{~S}_{4}$ samples.

The 100 liter anaerobic reactor produced $>50 \mathrm{~g}$ of $\mathrm{ZnS}$ during the first batch run. The actual amount of $\mathrm{ZnS}$ produced during this process was $\sim 62 \mathrm{~g}$ and the crystallite size was about $4.9 \mathrm{~nm}$. This was very important for the scale up efforts required to achieve large amounts of NF-nanoparticles. The potential for using nanofermentation to produce nanoparticles without any effects on the size of the nanoparticles is crucial for both optoelectronics and sensor applications.

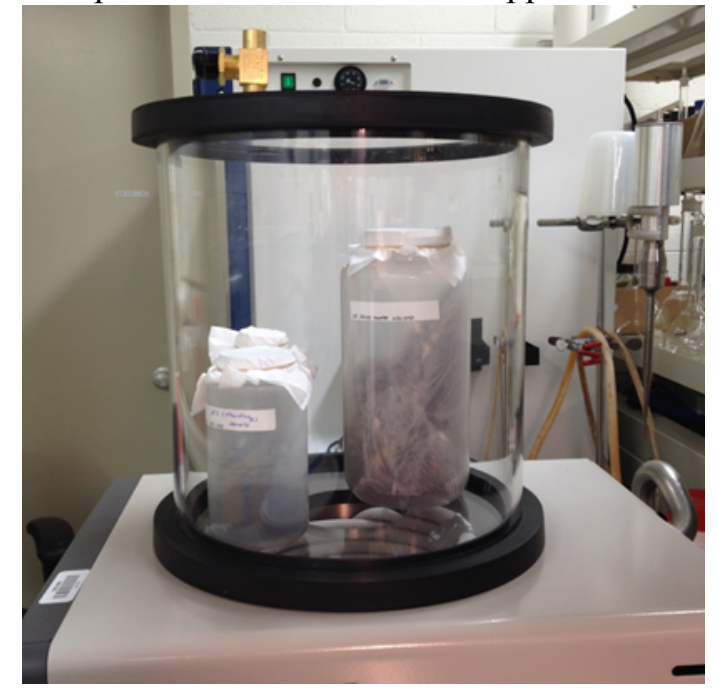

Figure 1.22. Freeze drying condensed zinc sulfide sample at $>50 \mathrm{~g}$ amount from the 100 liter reactor.

\subsubsection{Metal Oxide Coatings by Nanofermentation Route}

The final subtask is focused on the development of metal oxide coatings using n-type $\mathrm{ZnO}$ and p-type $\mathrm{NiO}$ nanoparticles synthesized by nanofermentation (NF) bioprocessing method.

\subsubsection{ZnO coatings and thin films}

To improve the $\mathrm{ZnO}$ coating quality, different capping agents and post-washing treatments were investigated. The surface capping agents were expected to enhance particle agglomeration control during synthesis and increase ink loading without compromising ink quality. The post-washing treatments were aimed at elimination of surface groups controlling particle agglomeration and dispersion.

The $\mathrm{ZnO}$ thin films have been developed by pulsed laser deposition technique using a $\mathrm{ZnO}$ target processed from NF $\mathrm{ZnO}$ particles. The $\mathrm{ZnO}$ thin film performance has been evaluated in terms of the structural, optical, and electrical characteristics.

The Low Temperature Materials Synthesis team supplied us with another batch of $\mathrm{ZnS}(>20 \mathrm{~g})$ precursors for sensor as-synthesized. These precursors can be also be converted to $\mathrm{ZnO}$ through a controlled annealing process. Two zinc sulfides samples (Figure 1.23, right two samples) were newly synthesized and compared to the other two samples (Figure 1.23, left two samples) synthesized from a continuous batch using MOPS buffer and multiple batches involving HEPES buffer. After harvesting and freeze-drying the appearance of the latest samples exhibited a brighter color on the surface than previously produced samples. This nanofermentation process required only a 24 hour 
reaction period which indicates that the modified production protocol with the reduced reaction time can play a role in improving the production rate.

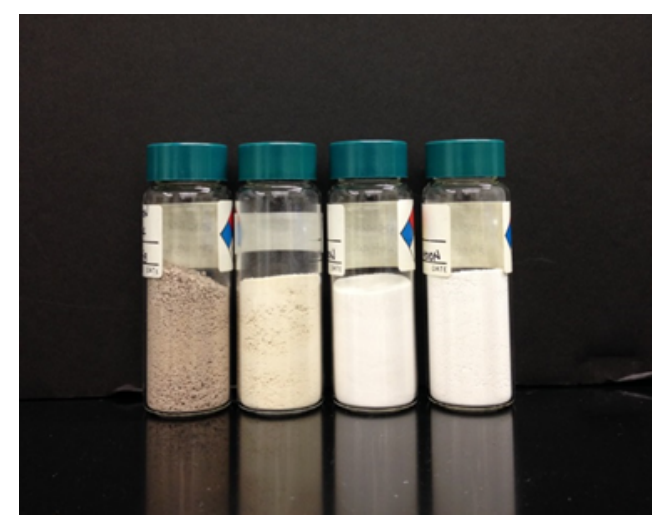

Figure 1.23. The latest batch of ZnS NF-nanoparticles produced in the Low Temperature Materials Synthesis project.

\subsection{INK DEVELOPMENT}

The nanoparticle ink development task involved the following critical steps:

$\rightarrow$ Characterization of the $\mathrm{ZnS}$ nanoparticle (NP) surface

$\rightarrow$ Identification of Sonospray deposition requirements and translate into nanoparticle properties and ink characteristics required for successful deposition

$\rightarrow$ Defining the amount of NPs required for deposition and ink development studies

$\rightarrow$ Determining range of Sono-spray solution solids loadings for NPs

$\rightarrow$ Identification of potential additives to adjust dispersion, flow requirements, processing aids, etc., of ink formulation without contamination of final thin film

The initial task work on this project, involved the purchase of commercial $\mathrm{ZnS}$ nanoparticles $(\sim 10 \mu \mathrm{m}$ size), which were used as a baseline reference to determine applicable solvent chemistry and approximate coating quality for micron-sized particles. The following steps were initiated in the characterization of commercial and NF ZnS raw material for ink development:

- Establishing powder size distribution and surface chemistry

- Measuring nanoparticle size

- Determining the zeta potential

- $\quad$ Measuring the surface area

The initial candidate dispersants for NF ZnS were evaluated based on the following characterization of commercial and NF powders/solutions:

- Sedimentation

- Zeta potential

- Rheology

\subsubsection{Commercial ZnS Zeta Potential Results}

The zeta potential of a system is a measure of charge stability, and controls all particle-particle interactions within a suspension. Measuring the zeta potential of particles in suspension is used to predict stability and behavior when exposed to changes in ionic strength of the solution. When 
nanoparticles are suspended in a fluid, zeta potential maintains the dispersion or discreteness of the particles in suspension. Understanding zeta potential is of critical importance in controlling dispersion and determining the stability of a nanoparticle suspension, i.e. to what degree aggregation will occur over time. The zeta potential is the measure of the electric potential at the slip plane between the bound layer of diluent molecules surrounding the particle, and the bulk solution. This can be closely linked to the particle's surface charge in simple systems, but is also heavily dependent on the properties of the diluent solution. A higher level of zeta potential results in greater electrostatic repulsion between the particles, minimizing aggregation/flocculation. The nanoparticle ink development was carried out in close correlation between zeta potential and $\mathrm{pH}$ of the ink. Figure 1.24 shows the initial results on commercial ZnS particle based ink. First results indicated that a cationic dispersant, such as polyethyleneimine (PEI), appears to be a good candidate for the sedimentation studies (Isoelectrical Point (IEP) is $\leq 4$ ).

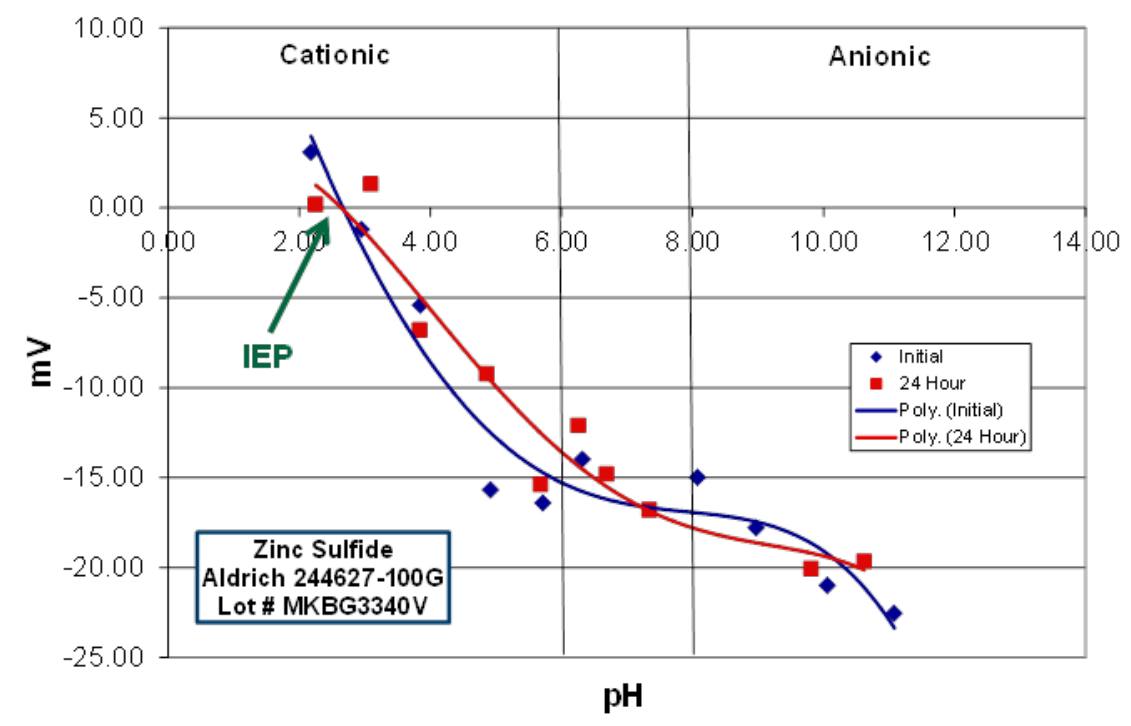

Figure 1.24. Relation between the zeta potential and $\mathrm{pH}$ of the ink using polyethyleneimine.

\subsubsection{Dispersion of NF ZnS Particles}

The development of a NF derived ZnS slurry was built on the zeta potential characterization that was completed on commercially produced $\mathrm{ZnS}$ powder. These results showed that the commercially derived $\mathrm{ZnS}$ had an Isoelectrical Point (IEP) 3. As the NF ZnS is received in a solution of water, and assuming the surfaces of the commercially derived and NF ZnS will be similar to each other, suspension of the NF ZnS in this solution was facilitated by the use of a cationic dispersant. To balance the size of the NF ZnS with an equivalent molecular weight (MW) of a typical cationic dispersant, three low molecular weights were selected. An effective way to quickly evaluate the stability of powders in solution is a sedimentations study. Simply, the sediment height is measured as a function of time. The longer powders stay in the suspension without settling, the more stable the suspension. Dispersants can be added to the solutions to examine the efficacy of the dispersant as well as study the effect of the ratio of the dispersant to the solids by weight. For the NF ZnS sedimentations studies, 600, 1200, and $1800 \mathrm{MWs}$ of polyethyleneimine (PEI) were selected. In general, polyethyleneimine (PEI) was able to suspend NF ZnS at a minimum concentration over a 24hour period. The settling curves and the minimum concentrations for 600,1200 and $1800 \mathrm{MW}$ are shown in Fig. 1.25 through 1.27 with concentrations of 51, 36, and 37\%, respectively. NF ZnS slurries were provided for evaluation in the coating studies for each of these minimum concentrations. 
In these studies, dilute ( $\sim 1 \mathrm{wt} \%$ solids) NF ZnS slurries were developed utilizing a cationic dispersant polyethyleneimine (PEI). Film formation of the dilute slurries showed several developmental issues that still needed to be addressed. The dilute nature of the slurries did not allow for dense film formation upon heat treatment. Slurries with higher solids loadings were required. In addition, there was concern that the high concentrations of PEI used relative to the actual solids of $\mathrm{ZnS}$ were leaving behind a carbonaceous ash upon heat treatment. As a result of that concern, the focus for the next stage if the work was to determine the level of ash left behind from the PEI, determine if it was detrimental to the final film formation, and in parallel search for an alternative dispersion approach for the PEI.

\section{Weight\% of $600 \mathrm{MW}$ PEI}

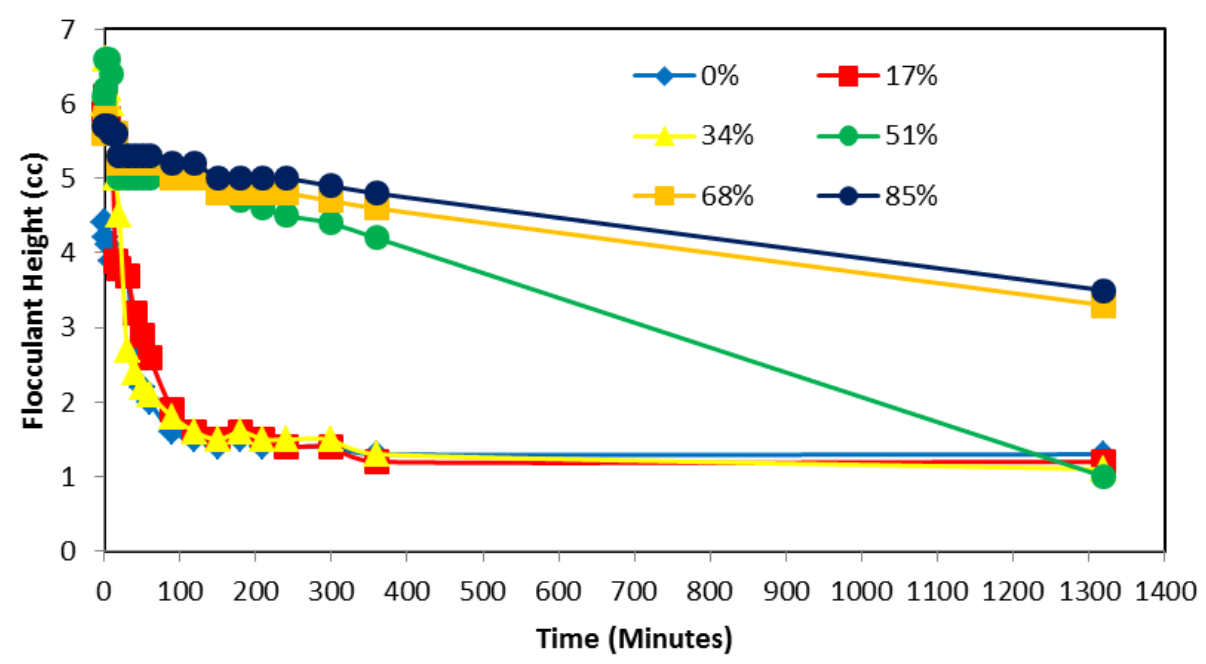

Figure 1.25. Sedimentation Curve for Additions of 600 MW PEI in an Aqueous Solution of 1 weight \% NF ZnS.

\section{Weight \% of 1200 MW PEI}

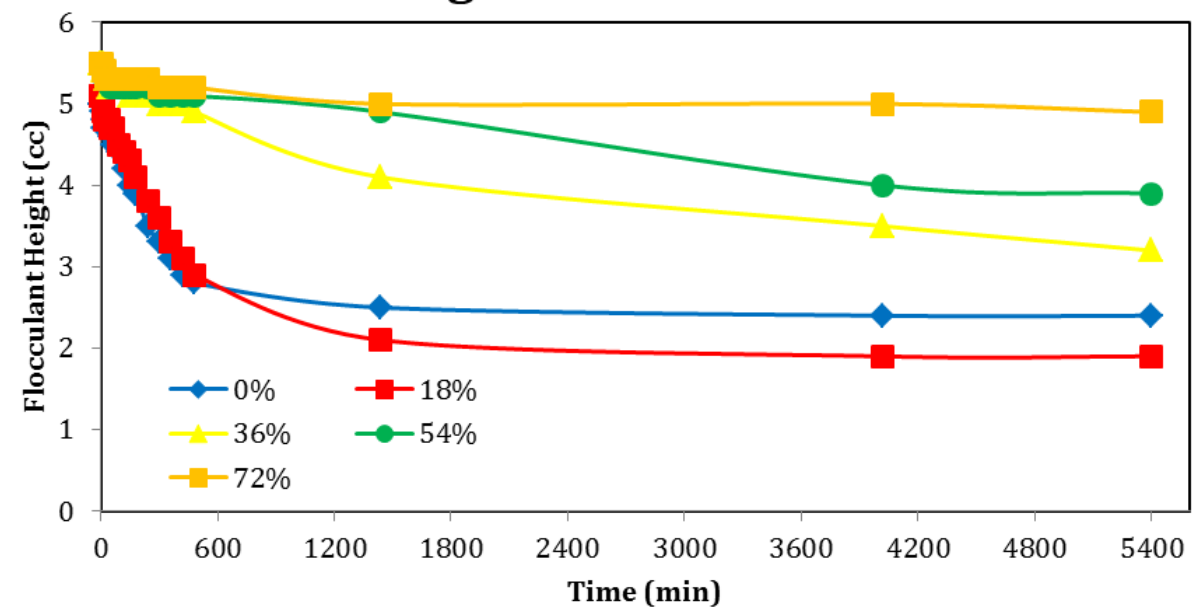

Figure 1.26. Sedimentation Curve for Additions of 1200 MW PEI in an Aqueous Solution of 1 weight \% NF ZnS. 


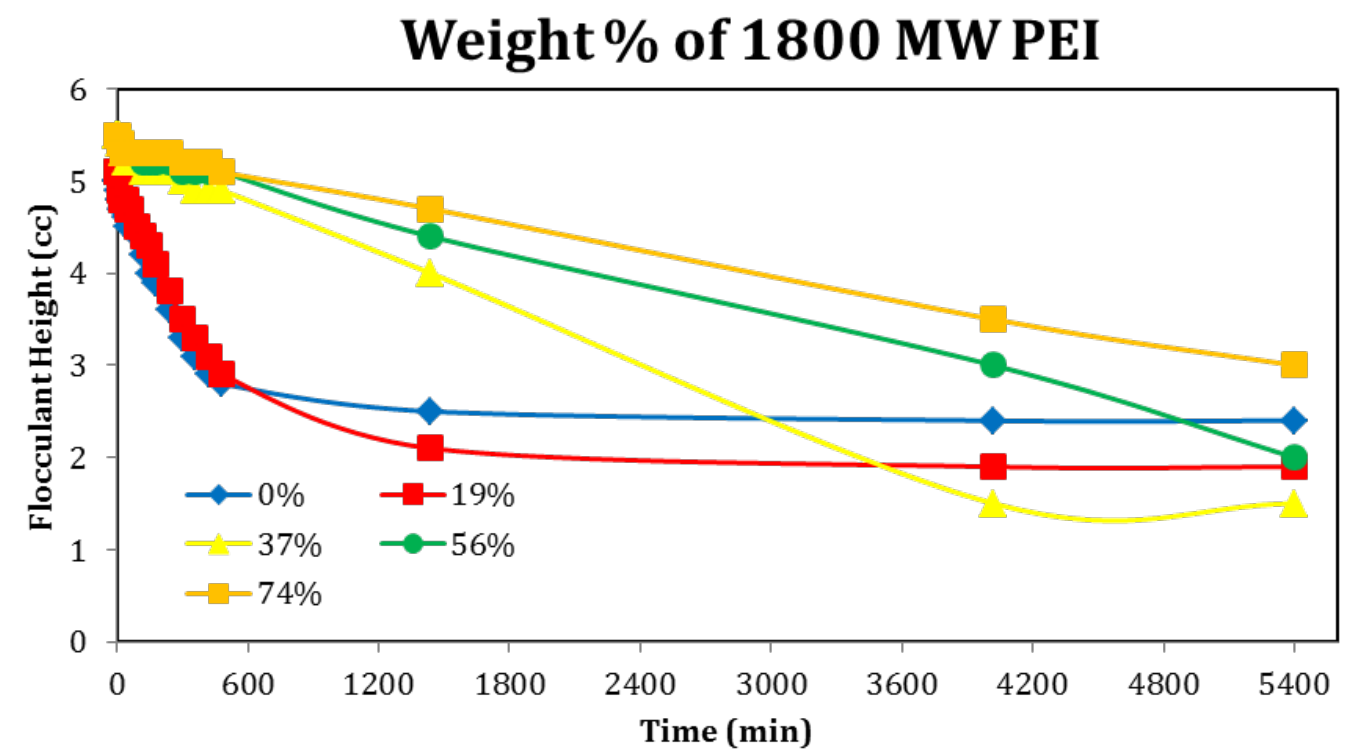

Figure 1.27. Sedimentation Curve for Additions of 1800 MW PEI in an Aqueous Solution of 1 weight \% NF ZnS.

To determine the amount of carbonaceous material on the surface of the $\mathrm{ZnS}$ particles, thermal gravimetric analysis (TGA) was run in both air and argon environments of slurries of both stabilized and unstabilized (as-synthesized) NF ZnS slurries. Figures 1.28 and 1.29 show the weight loss curves as a function of temperature in air and argon, respectively. There were differences in the weight loss depending on whether the sample is heated in air or in argon. When argon was used, the major weight loss occurs between $200^{\circ} \mathrm{C}$ and $400^{\circ} \mathrm{C}$. Also there was additional weight lost above $800^{\circ} \mathrm{C}$ and the rate was increasing as the temperature increased above $800^{\circ} \mathrm{C}$. There was no heating above $1000^{\circ} \mathrm{C}$, nor hold at $1000^{\circ} \mathrm{C}$, so the final weight loss in argon was not determined. When air was used, the "as received $\mathrm{ZnS}$ " had weight loss between $200-400^{\circ} \mathrm{C}$ and again between 550 and $650^{\circ} \mathrm{C}$. The overall weight loss was about $34 \%$. Above $800^{\circ} \mathrm{C}$ there was a slight weight gain - not a weight loss as exhibited when run in argon. The samples containing the PEI dispersant exhibited several regions between 200 and $700^{\circ} \mathrm{C}$ where the rate of weight loss reached maximum values. What can be seen in both weight loss curves irrespective of atmosphere is that there is carbonaceous material present on the surface of the as-synthesized powders. This is residue from the fermentation process, which motivated the efforts described above to reduce the organic content. The full effect of this excess carbon was examined more closely to determine if it impacted the processing or electronic properties in the final film. In addition, the high temperatures required to remove the PEI and the amount of ash remaining after heat treatment due to the large amounts of PEI utilized to stabilize the slurry may also be detrimental to final electronic properties. As a result, alternative approaches to stabilization were examined. 


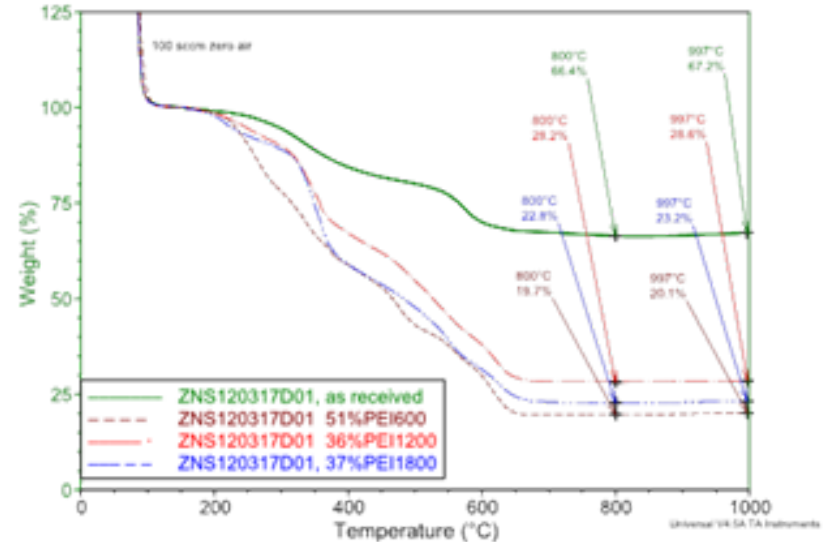

Figure 1.28. Weight loss cures of $\mathrm{NF} \mathrm{ZnS}$ solutions in air with and without the use of the dispersant PEI.

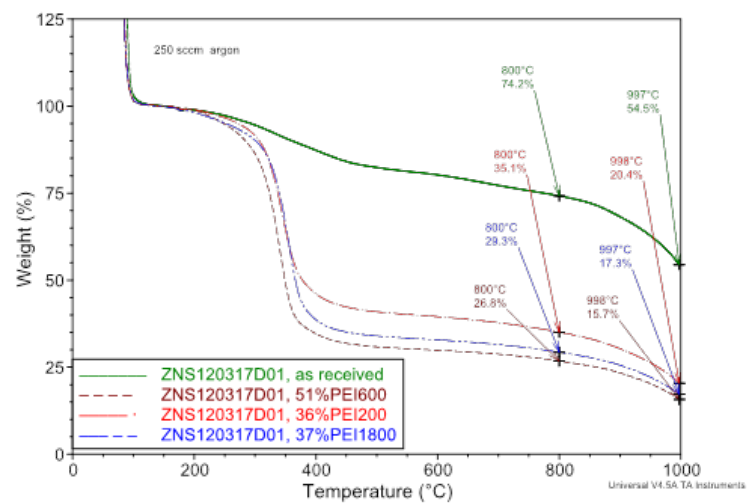

Figure 1.29. Weight loss cures of NF ZnS solutions in argon with and without the use of the dispersant PEI.

Initially, zeta potential of commercial $\mathrm{ZnS}$ powders (Aldrich) was characterized while waiting for the NF ZnS solutions to be ready. Stabilization approaches were based on the results of the commercial powder. As larger quantities of the NF ZnS solutions became available for characterization, the zeta potential was re-measured using the NF materials. The zeta potential comparison between the two powder sources is shown in Figure 1.30. The Aldrich $\mathrm{ZnS}$ has a very low isoelectric point requiring a cationic dispersant for stabilization whereas the NF ZnS's isoelectric point is higher. The NF ZnS still can utilize a cationic dispersant, but an anionic dispersant could also be used due to the more neutral value of its isoelectric point. It is believed the difference in the isoelectric point is due to the carbonaceous material seen on the surface of the $\mathrm{ZnS}$ powder from the TGA experiments.

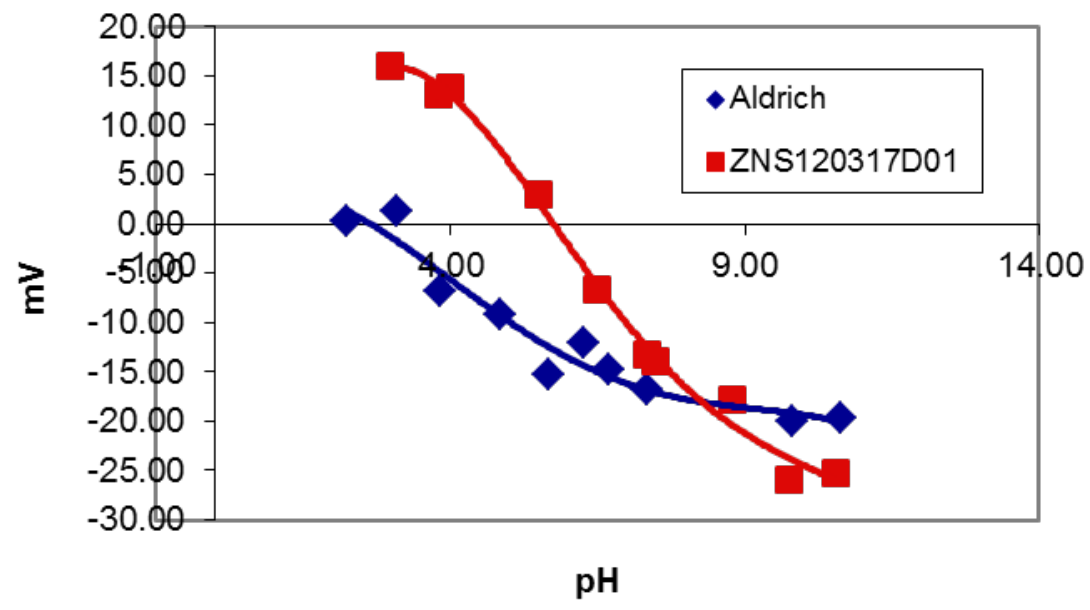

Figure 1.30. Zeta potential curves of commercial and NF ZnS powders.

To evaluate the effect of charge stabilization versus steric stabilization ( $\mathrm{pH}$ control versus the use of a polymeric dispersant), a sedimentation study was conducted where a NF ZnS slurry pH was left 
unadjusted (control), adjusted to a $\mathrm{pH}$ of 3 or 10 and the addition of $1 \mathrm{wt} \%$ oleic acid by solids weight was added. Oleic acid was chosen as a replacement dispersant for PEI due to its low burnout temperatures $\left(<300^{\circ} \mathrm{C}\right)$. The resulting sedimentation behavior as a function of time is shown in Figure 1.31. The charge stabilized conditions, $\mathrm{pH} 3$ and 10, were ineffective in preventing powder settling even over short time periods. Oleic acid seemed to be a slight improvement over natural solution.

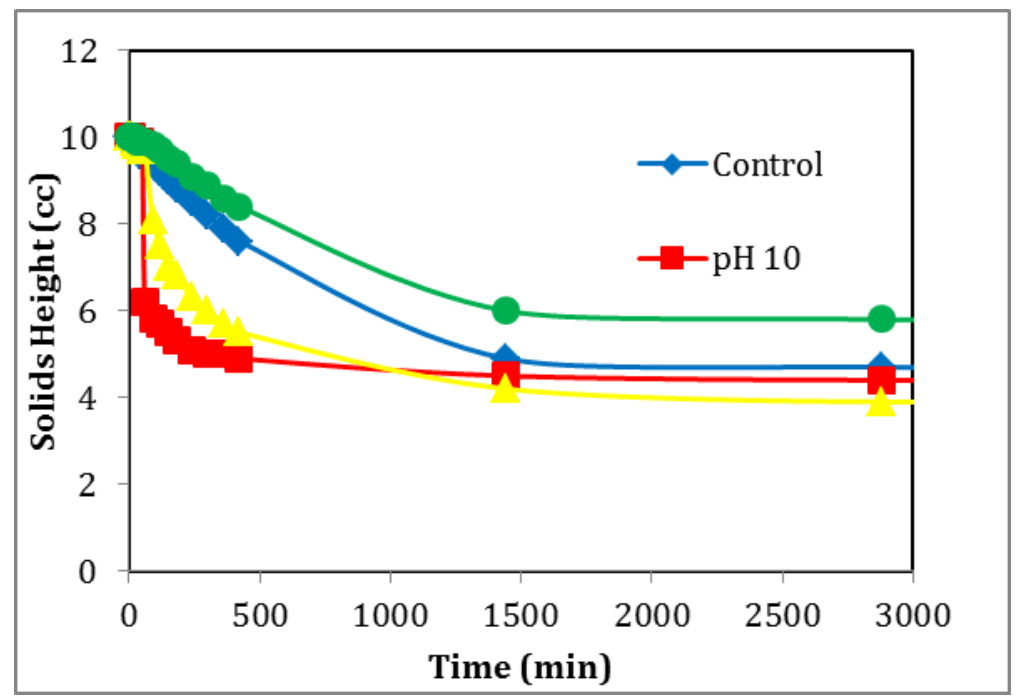

Figure 1.31. Sedimentation curve for alternative dispersion approaches for NF $\mathrm{ZnS}$ in an aqueous solution.

These dispersion studies of the NF zinc sulfide powders showed oleic acid to be a slight improvement in sedimentation studies as a candidate replacement dispersant for PEI. PEI, although effective, is burned out, i.e., removed by heating and the subsequent break down and volatization of the organic material, at relatively high temperatures $(>400 \mathrm{C})$. To maintain the nanoparticulate nature of the starting materials by limiting heat treatment temperatures that might promote grain growth and reduce the opto-electronic properties of the final film, a search was initiated to identify dispersants that may decompose at lower temperatures than PEI.

Initially, a $10 \%$ by weight solution of NF $\mathrm{ZnS}$ was used as the base for the dispersant studies. A wide variety of candidate dispersants were selected and evaluated for effectiveness. The dispersants were chosen based on decomposition temperature and/or compatibility to the starting chemistry. Each candidate was added to an aliquot of the base $\mathrm{ZnS}$ solution, mixed ultrasonically, and the resulting particle size of the solution was measured using a laser light scattering technique. Generally, if the average particle size (D50) was minimized, and the total size distribution was tight (the minimum range from the D10 to the D90), the dispersant was flagged for further consideration. Table 1.5 shows the results from the initial dispersant particle size study. The dispersant Triton X-100 (polyethylene glycol octyphenyl ether) showed the best improvement and the tightest spread in particle size overall, but as this technique was used just for preliminary screening, four additional dispersants from this group of 13 were selected for further study. They were ethylenediaminetetraacetic acid (EDTA), nitrilotriacetic acid (NTA), glycine (GLY), and sodium lignosulphonate (Lignotech Vanisperse CB). Polyethylene glycol (PEG) and oleic acid were also included. 
Table 1.5 Particle Size Results for NF ZnS Dispersant Screening Study

\begin{tabular}{|c|c|c|c|c|}
\hline Chemical Name & $\begin{array}{l}\text { Mean Size } \\
(\mu \mathrm{m})\end{array}$ & $\begin{array}{l}\text { Diameter on } \\
\text { cumulative } \\
10 \%\end{array}$ & $\begin{array}{l}\text { Diameter on } \\
\text { cumulative } \\
\mathbf{5 0 \%}\end{array}$ & $\begin{array}{l}\text { Diameter on } \\
\text { cumulative } \\
90 \%\end{array}$ \\
\hline $\begin{array}{l}\text { Glacial Acetic } \\
\text { Acid }\end{array}$ & 1.42591 & 0.6884 & 1.2828 & 2.3318 \\
\hline $\begin{array}{l}\text { Alginic Acid } \\
\text { Sodium Salt }\end{array}$ & 2.62987 & 1.0266 & 2.2449 & 4.7957 \\
\hline Aquazol 200 & 0.75057 & 0.2550 & 0.6401 & 1.3803 \\
\hline EDTA & 1.02373 & 0.1903 & 0.3009 & 2.9656 \\
\hline $\begin{array}{l}\text { EDTA Disodium } \\
\text { Salt }\end{array}$ & 52.62920 & 0.2844 & 55.6674 & 107.4003 \\
\hline $\begin{array}{l}\text { EDTA Magnesium } \\
\text { Disodium Salt }\end{array}$ & 1.50506 & 0.7352 & 1.3327 & 2.4741 \\
\hline Glycine & 18.85070 & 0.7870 & 2.2372 & 87.6004 \\
\hline Isopropyl Myristate & 1.1479 & 0.4397 & 0.9921 & 2.0257 \\
\hline $\begin{array}{l}\text { Lignotech } \\
\text { Vanisperse CB }\end{array}$ & 0.68462 & 0.1935 & 0.2981 & 1.3157 \\
\hline $\begin{array}{l}\text { Nitrilotriacetic } \\
\text { Acid }\end{array}$ & 0.44343 & 0.1624 & 0.2417 & 0.5823 \\
\hline Polyacrylic Acid & 23.77108 & 1.3172 & 3.7303 & 84.5252 \\
\hline PVA $14,000 \mathrm{MW}$ & 63.22652 & 2.4985 & 64.4539 & 106.9646 \\
\hline Triton X-100 & 0.26456 & 0.1717 & 0.2460 & 0.3722 \\
\hline
\end{tabular}

In the first dispersant evaluation, the dispersants were added post-synthesis, that is they were added after the nanofermentation process was completed and the particles were harvested. The dispersants were effective, but the goal was to further reduce the agglomerate/particle size of the NF ZnS powder. One hypothesis was that the NF ZnS particles may be agglomerating while they growing in solution. If a dispersant can be utilized during synthesis, agglomeration may be able to be further controlled and minimized. Therefore, a second set of particle size measurements were run on the seven dispersants selected post-synthesis for evaluation as in-situ dispersants, and the results are given in Table 1.6. 
Table 1.6 Particle Size Results for NF ZnS In-Situ Dispersant Study

\begin{tabular}{|l|l|l|l|l|l|}
\hline \multirow{2}{*}{ Dispersant } & \multirow{4}{*}{ Sonicated } & \multirow{4}{|l|}{ Sizes $(\mu \mathrm{m})$} \\
\cline { 3 - 6 } & & Mean & $10 \%$ & $50 \%$ & $90 \%$ \\
\cline { 3 - 6 } & & 27.51362 & 1.4292 & 4.1024 & 91.6895 \\
\hline NTA & Yes & 42.33607 & 0.8939 & 9.0422 & 106.9210 \\
\hline PEG & & 3.67071 & 0.9054 & 1.7291 & 3.8481 \\
\hline PEG & Yes & 0.78509 & 0.2427 & 0.6549 & 1.4812 \\
\hline GLY & & 3.46347 & 0.8943 & 1.6846 & 3.7526 \\
\hline GLY & Yes & 43.22005 & 0.4518 & 44.5329 & 90.1340 \\
\hline Oleic Acid & & 1.89081 & 0.8453 & 1.6176 & 3.2741 \\
\hline Oleic Acid & Yes & 31.72499 & 0.3629 & 2.2040 & 90.8515 \\
\cline { 3 - 6 } EDTA & & 3.12265 & 1.3719 & 2.7984 & 5.3282 \\
\hline EDTA & Yes & 2.51732 & 0.7856 & 2.0624 & 4.8778 \\
\hline Triton X-100 & & 33.08715 & 1.3137 & 3.7612 & 112.8235 \\
\hline Triton X-100 & Yes & 1.13278 & 0.3448 & 0.9436 & 2.1266 \\
\hline Lignosulfonate & & 72.68216 & 0.2780 & 59.7383 & 168.7897 \\
\hline Lignosulfonate & Yes & 90.53172 & 0.2356 & 67.7824 & 209.2655 \\
\hline
\end{tabular}

The results shown in Table 1.6 were encouraging. Similar results were seen for the Triton X-100, and an even smaller size and distribution were seen for PEG. Again, as this was a screening tool to assist in down selecting an effective dispersant, no optimization of the dispersant in terms of concentration of dispersant to surface area of the NF ZnS or the molecular weight of the PEG to the size of the particle had been completed yet. Thus, a third study was run where the concentration of the PEG was varied during synthesis from 0.05 to 5.0 weight $\%$, a normal dispersant concentration range for colloidal to submicron particles in suspension. Again, particle size was measured on the resulting solutions, and the results are given in Table 1.7. The most reduction in particle size is seen between 0.1 and 0.5 weight $\%$ PEG. Larger concentrations show larger agglomeration indicating entangling of the PEG strands causing larger agglomerates.

Table 1.7 Particle Size Results for NF ZnS- PEG Concentration Study

\begin{tabular}{|l|l|l|l|l|l|}
\hline \multirow{2}{*}{ Dispersant } & \multirow{2}{*}{ Sonicated } & & \multicolumn{4}{l|}{ Sizes $(\mu \mathrm{m})$} & \multicolumn{4}{|l|}{ Diameter on Cumulative \% } \\
\cline { 3 - 6 } & & Mean & $10 \%$ & $50 \%$ & $90 \%$ \\
\hline PEG & & 2.77971 & 1.2154 & 2.4258 & 4.8458 \\
\hline $\begin{array}{l}0.05 \% \\
\text { PEG }\end{array}$ & & 1.94941 & 0.4993 & 1.5940 & 3.8435 \\
\hline $\begin{array}{l}0.05 \% \\
\text { PEG }\end{array}$ & Yes & 2.05337 & 0.9134 & 1.7585 & 3.5674 \\
\hline $0.1 \%$ PEG & & & & & \\
\hline
\end{tabular}




\begin{tabular}{|l|l|l|l|l|l|}
\hline $0.1 \%$ PEG & Yes & 1.20252 & 0.3969 & 1.0074 & 2.2012 \\
\hline $0.5 \%$ PEG & & 2.54345 & 1.0496 & 2.1122 & 4.5935 \\
\hline $0.5 \%$ PEG & Yes & 1.44521 & 0.4639 & 1.1374 & 2.6727 \\
\hline $1.0 \%$ PEG & & 2.24235 & 0.9809 & 1.8865 & 3.9429 \\
\hline $1.0 \%$ PEG & Yes & 782.19592 & 442.1594 & 843.0077 & 969.5259 \\
\hline $5.0 \%$ PEG & & 1.94428 & 0.8283 & 1.6328 & 3.4144 \\
\hline $5.0 \%$ PEG & Yes & 805.37909 & 628.0833 & 814.9722 & 964.2176 \\
\hline
\end{tabular}

\subsubsection{Zn Gallate Nanoparticle Synthesis}

The $\mathrm{Zn}$ Gallate nanoparticle synthesis process was developed for low-cost, high performance phosphor applications. The ink development process was optimized for particle size control in the sub-micron range while larger particles were used for passive device development. The $\mathrm{Zn}$ Gallate material performance was evaluated in terms of the ink stability, coating quality control, particle crystallinity, PL response of intrinsic/doped coatings, and electrical conductivity. The sample splits for the investigation are listed in Table 1.8. The initial sample set included various dopants to analyze their impact on the PL emission characteristics.

Table 1.8 Zinc Gallate sample splits

\begin{tabular}{|c|c|c|}
\hline Particle Type & Sample ID & Sample Description \\
\hline Zn Gallate & 120529_A01 & $\begin{array}{l}\text { FeS medium, precursor with Co } 1 \% \text { replacing } \mathrm{Zn}-1 \text { wk incubation } \\
\text { Freeze dried }(1.686 \mathrm{~g} / 3 \mathrm{~L}) \\
\text { With sintering ( } 30 \mathrm{~min} \text { in air }+30 \mathrm{~min} \text { in nitrogen gas) should show strengthened BLUE }\end{array}$ \\
\hline Zn Gallate & 120529_B01 & $\begin{array}{l}\text { FeS medium, precursor with Mn } 4 \% \text { replacing Ga-1wk incubation } \\
\text { Freeze dried }(2.015 \mathrm{~g} / 3 \mathrm{~L}) \\
\text { With sintering ( } 30 \text { min in air) should show GREEN }\end{array}$ \\
\hline Zn Gallate & 120529_C01 & $\begin{array}{l}\text { FeS medium, precursor with } \mathrm{Cr} 1 \% \text { replacing Ga-1wk incubation } \\
\text { Freeze dried }(1.875 \mathrm{~g} / 3 \mathrm{~L}) \\
\text { With sintering ( } 30 \mathrm{~min} \text { in air) should show RED }\end{array}$ \\
\hline Zn Gallate & 120711_A01 & $\begin{array}{l}\text { FeS medium, precursor with Mn } 4 \% \text { replacing } \mathrm{Zn}-1 \text { wk incubation } \\
\text { Freeze dried }(1.574 \mathrm{~g} / 2 \mathrm{~L} \text { ) with Gallium } \\
\text { chloride } \\
\text { With sintering ( } 30 \mathrm{~min} \text { in air) should show GREEN }\end{array}$ \\
\hline Zn Gallate & 120711_B01 & $\begin{array}{l}\text { FeS medium, precursor with Mn } 4 \% \text { replacing } \mathrm{Zn}-1 \text { wk incubation } \\
\text { Freeze dried }(0.897 \mathrm{~g} / \mathrm{L}) \text { with Gallium Nitrate } \\
\text { Hydrate } \\
\text { With sintering ( } 30 \mathrm{~min} \text { in air) should show GREEN }\end{array}$ \\
\hline
\end{tabular}

\subsubsection{Ultrasonic Excitation}

The next task was to disperse nanoparticle agglomerates and increase the shelf life of inks and pastes through ultrasonic excitation. The primary surfactant used for paste development was nitrilotriacetic acid (NTA) at concentrations between 0.05 and 1.0\%. Ultrasonic excitation was considered as a postsynthesis approach to physically break up agglomerate structures, both immediately following 
synthesis as well as following storage. Bottled samples were placed in an ultrasonic bath and exposed to frequencies of 40,170, and $270 \mathrm{kHz}$. The frequency $40 \mathrm{kHz}$ worked the best because it reduced the particle size from $378.6 \mathrm{~nm}$ to $177.3 \mathrm{~nm}$ in one hour (Figure 1.32). Further testing of the $40 \mathrm{~Hz}$ frequency revealed that increasing the time to two hours yielded a small decrease in particle size. The same test run at $40 \mathrm{~Hz}$ for one hour in an ice bath yielded a particle size of $192.5 \mathrm{~nm}$, which was larger than that generated for one hour in water of unregulated temperature.

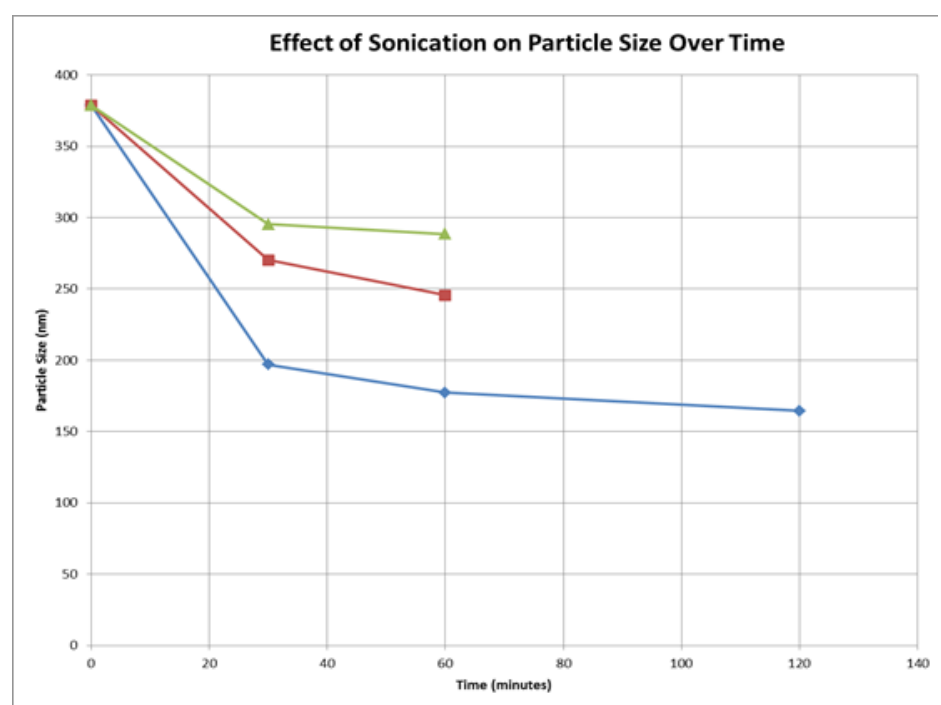

Figure 1.32. Particle size as a function of ultrasonic excitation exposure.

\subsubsection{Oxidation of $\mathrm{NF}$ metal sulfide (ZnS, NiS, $\mathrm{SnS}, \mathrm{CoS}$, and $\mathrm{Cu}$ ) particles}

In the next phase of the study, metal sulfide samples were produced ( $\mathrm{ZnS}, \mathrm{NiS}, \mathrm{SnS}, \mathrm{CoS}$, and $\mathrm{Cu}$ ) and were oxidized in either air or oxygen environments over a range of temperatures to determine the optimum heat treatment conditions to convert the sulfide materials to their oxide forms. The resulting powders were analyzed using $\mathrm{x}$-ray diffraction to determine the phases formed during the heat treatment (see Figure 1.33). 

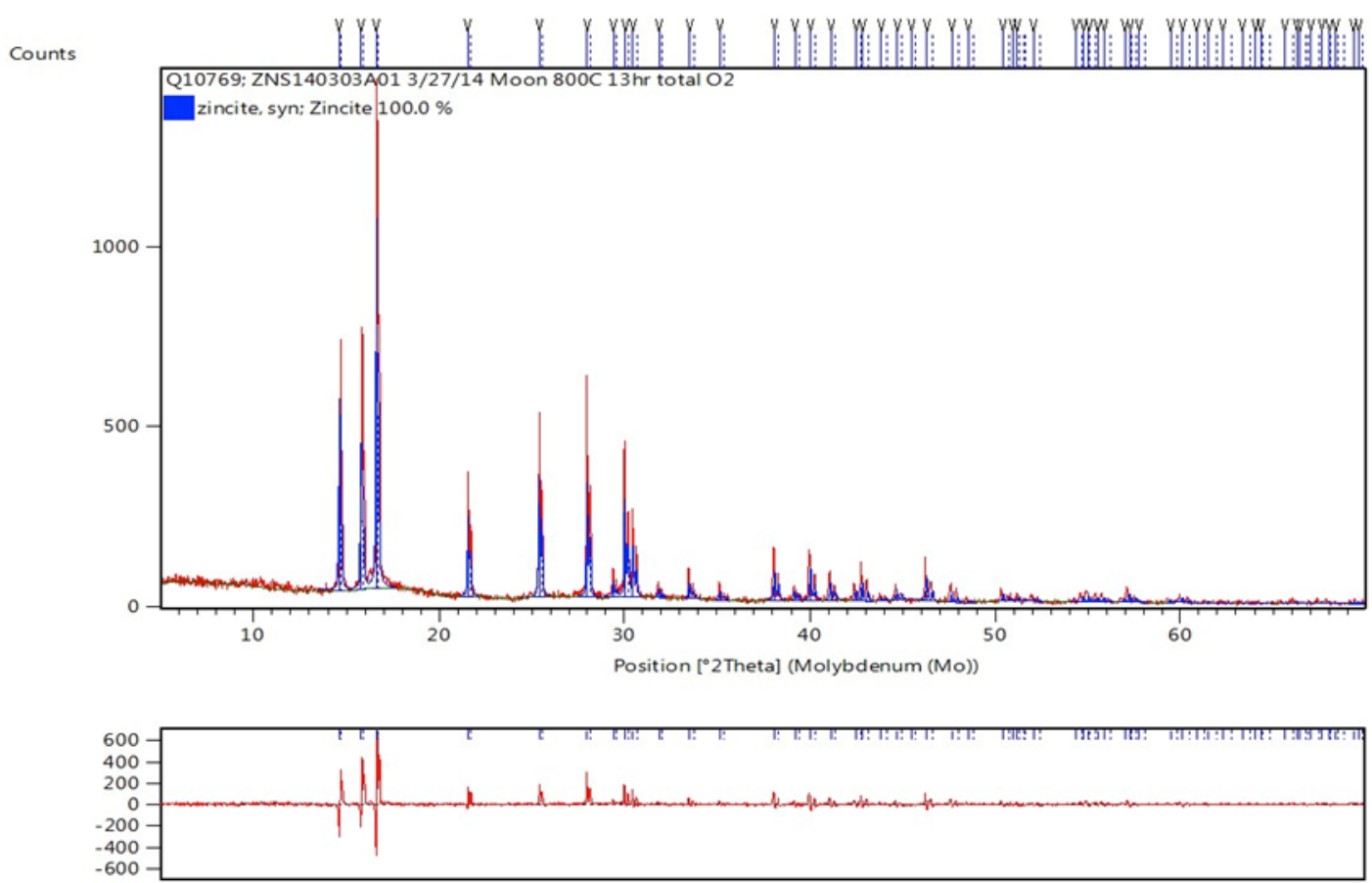

Figure 1.33. The $\mathrm{ZnS}$ was converted to $\mathrm{ZnO}$ with oxidation in $\mathrm{O}_{2}$ atmosphere at $800^{\circ} \mathrm{C}$ for $1 \mathrm{hour}$.

Subsequently, a set of $\mathrm{ZnO}$ coatings was prepared for conductivity measurements. Two types of $\mathrm{ZnO}$ powder were used, one exposed to laboratory air or one exposed to pure oxygen. Both witnessed heat treatment conditions of $600^{\circ} \mathrm{C}$ for 1 hour. The coatings were fabricated by pressing the powder onto an adhesive $\mathrm{Cu}$ substrate. The $\mathrm{Cu}$ substrate will be used as an electrode for the conductivity measurements. A stencil ( $5 \mathrm{~mm} \times 5 \mathrm{~mm} \times 12 \mu \mathrm{m})$ was placed on top of the $\mathrm{Cu}$ to create a coated area with specific dimensions.

Fabrication of another set of $\mathrm{ZnO}$ and $\mathrm{SnO}$ coatings for conductivity measurements was initiated. This approach used screen-printing as the coating technique, and the screens were selected with the appropriate design. These included platinum screening printing paste for the electrode coating and a low temperature curing screen-printing paste vehicle from DuPont. The goal was to find either a conductive or a non-insulating coating material that will enable either no or low temperature heat treatment of the sensor materials such that flexible, polymer substrates could be utilized.

\subsubsection{Oxidation of NF metal sulfide ( $\mathrm{SnS}, \mathrm{CoS}_{2}, \mathrm{Ni}_{3} \mathrm{~S}_{4}$, and $\left.\mathrm{CuS}\right)$ particles}

The nanofermentation process was used for binary metal oxide particle synthesis and coating development. Nanofermentation process is efficient in the development of metal sulfide particles, which can be oxidized to form metal oxide particles for advanced sensor applications. In the present study, we have investigated the oxidation efficiency of four different NF metal sulfide particles: SnS, $\mathrm{CoS}_{2}, \mathrm{Ni}_{3} \mathrm{~S}_{4}$, and $\mathrm{CuS}$. The nanoparticles were annealed in a furnace in a flowing $\mathrm{O}_{2}$ atmosphere at various temperatures in the range of $400-800^{\circ} \mathrm{C}$. The metal-oxide structure was analyzed by $\mathrm{x}$-ray diffraction technique. As shown in Figure 1.34, it was possible to obtain a well-defined oxide phase at an annealing temperature of $800^{\circ} \mathrm{C}$ for all the metal-sulfides except $\mathrm{CoS}_{2}$. For $\mathrm{CoS}_{2}$ particles, a $\mathrm{CoSO}_{4}$ phase was also observed, as listed in Table 1.9, indicating incomplete removal of sulfur after 60 min of annealing at $800^{\circ} \mathrm{C}$. At an annealing temperature of $600^{\circ} \mathrm{C}$, only $\mathrm{SnS}$ was completely converted into the corresponding metal oxide. The XRD results indicated that a pure $\mathrm{SnO}_{2}$ phase was formed even after annealing at a temperature of $400^{\circ} \mathrm{C}$. The initial investigations focused on $\mathrm{SnO}_{2}$ 
particles for coating development. The impact of particle size and distribution on the coating quality were evaluated, and the coatings characterized in terms of structural, optical, and electrical characteristics. The resistive sensors were developed to analyze the gas sensing characteristics of $\mathrm{SnO}_{2}$ particles as a function of temperature.

The nanofermentation $\mathrm{ZnS}$ particles were oxidized to induce $\mathrm{ZnO}$ phase at an annealing temperature of $800^{\circ} \mathrm{C}$. The oxidation efficiency was found to strongly depend on the initial $\mathrm{ZnS}$ amount during annealing. It was possible to induce a pure $\mathrm{ZnO}$ phase even at an annealing temperature of $600^{\circ} \mathrm{C}$ for $<1 \mathrm{~g} \mathrm{ZnS}$ sample. However, impure $\mathrm{ZnO}$ phase was obtained even after annealing at $800^{\circ} \mathrm{C}$ when using $>5 \mathrm{~g} \mathrm{ZnS} \mathrm{sample} \mathrm{as} \mathrm{shown} \mathrm{in} \mathrm{Fig.} \mathrm{1.35.} \mathrm{The} \mathrm{phase} \mathrm{purity} \mathrm{did} \mathrm{not} \mathrm{improve} \mathrm{even} \mathrm{after} \mathrm{second}$ annealing at $800^{\circ} \mathrm{C}$ for $60 \mathrm{~min}$. On inspection, the furnace tube was found to be heavily coated by sulfur when using larger amounts of sulfur and the sulfur partial pressure was responsible for inefficient conversion of $\mathrm{ZnS}$ phase into $\mathrm{ZnO}$ phase. The tube was thoroughly cleaned prior to third anneal. As shown in Figure 1.35, a well-defined zincite phase was obtained after furnace tube cleaning, indicating that the oxidation efficiency was limited by sulfur partial pressure in the furnace rather than the annealing temperature. As a result of these observations, x-ray diffraction measurements were regularly conducted to maintain batch-to-batch control.
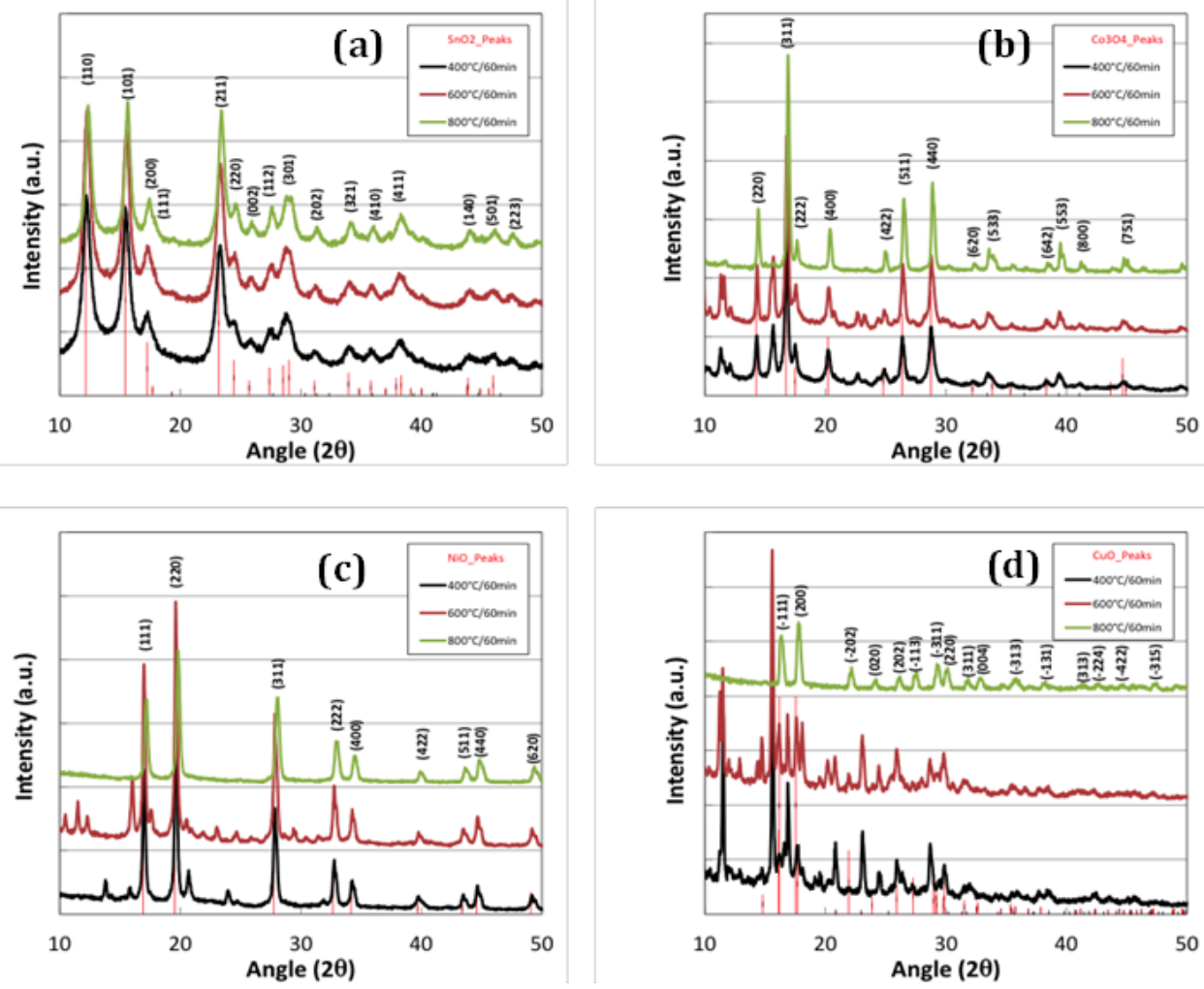

Figure 1.34. The $\mathrm{x}$-ray diffraction patterns showing the impact of $\mathrm{O}_{2}$ annealing on the metal oxide phase formation for (a) $\mathrm{SnS}$, (b) $\mathrm{CoS}_{2}$, (c) $\mathrm{Ni}_{3} \mathrm{~S}_{4}$, and (d) $\mathrm{CuS}$ particles. 
Table 1.9 The impact of $\mathrm{O}_{2}$ annealing on the metal oxide phase formation for (a) $\mathrm{SnS}$, (b) $\mathrm{CoS}_{2}$, (c) $\mathrm{Ni}_{3} \mathrm{~S}_{4}$, and (d) $\mathrm{CuS}$ particles.

\begin{tabular}{|c|c|c|c|c|c|}
\hline \multirow[b]{2}{*}{$\begin{array}{c}\text { Initial } \\
\text { Material }\end{array}$} & \multirow[b]{2}{*}{$\begin{array}{c}\text { Target } \\
\text { Composition }\end{array}$} & \multicolumn{3}{|c|}{ Annealing Temperature } & \\
\hline & & $400^{\circ} \mathrm{C}$ & $600^{\circ} \mathrm{C}$ & $800^{\circ} \mathrm{C}$ & \\
\hline $\begin{array}{l}\mathrm{SnS} \\
\mathrm{Ni}_{3} \mathrm{~S}_{4}\end{array}$ & $\begin{array}{l}\mathrm{SnO}_{2} \\
\mathrm{NiO}\end{array}$ & $\begin{array}{r}100 \% \\
80 \% \\
\text { Ni3\$2: } 5 \% \\
\text { NiS: } 15 \%\end{array}$ & $\begin{array}{r}100 \% \\
63 \% \\
\mathrm{Ni}(\mathrm{SO} 4): 37 \%\end{array}$ & $\begin{array}{r}100 \% \\
100 \%\end{array}$ & $--\frac{1}{3}$ \\
\hline Cus & $\mathrm{CuO}$ & $\begin{array}{r}8 \% \\
\mathrm{Cu}(\mathrm{SO} 4): 77 \% \\
\mathrm{Cu} 2 \mathrm{O}(\mathrm{SO} 4): \\
15 \%\end{array}$ & $\begin{array}{r}10 \% \\
\mathrm{Cu}(\mathrm{SO} 4): 48 \% \\
\mathrm{Cu} 2 \mathrm{O}(\mathrm{SO}) \mathrm{4}): \\
42 \%\end{array}$ & $100 \%$ & \\
\hline $\operatorname{CoS}_{2}$ & $\mathrm{CO}_{3} \mathrm{O}_{4}$ & $\begin{array}{l}44 \% \\
\mathrm{Co}(\mathrm{SO} 4) \\
{[\alpha-25 \%, \beta-32 \%]}\end{array}$ & $\begin{array}{l}41 \% \\
\operatorname{Co}(S O 4) \\
{[\alpha-36 \%, \beta-23 \%]}\end{array}$ & $\begin{array}{l}85 \% \\
\mathrm{Co}(\mathrm{SO} 4) \\
{[\alpha-15 \%,]}\end{array}$ & \\
\hline
\end{tabular}

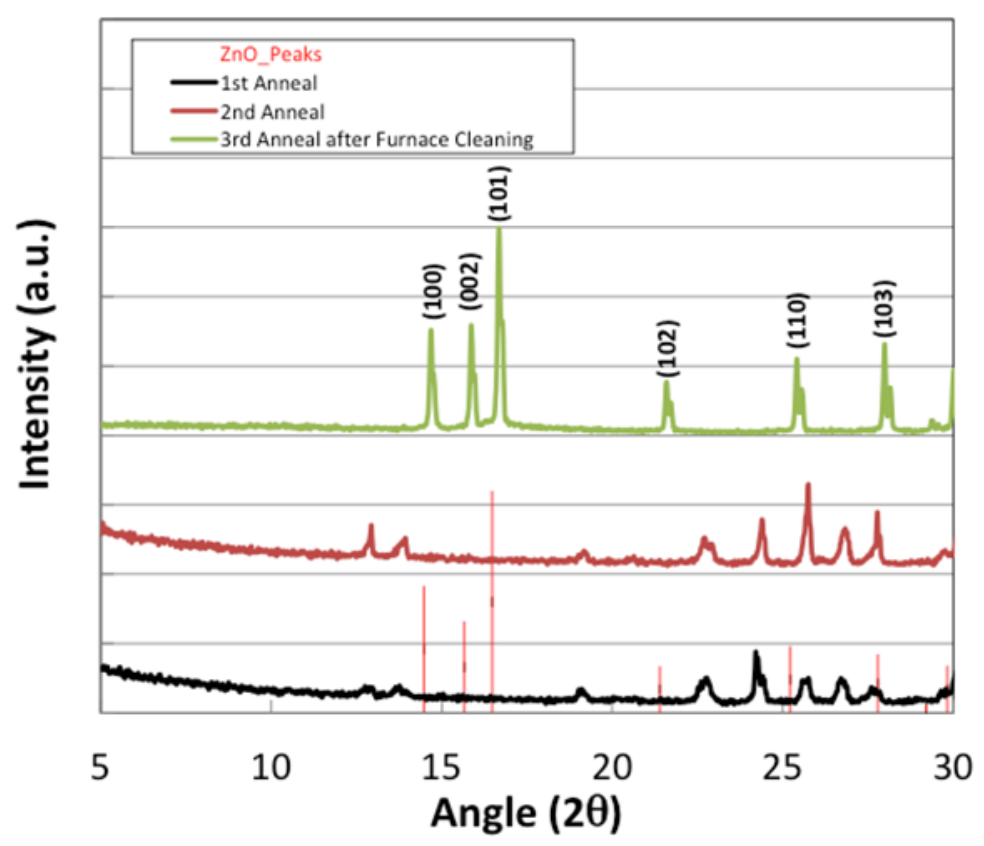

Figure 1.35. The impact of multiple anneals at $800^{\circ} \mathrm{C}$ on $\mathrm{ZnO}$ phase formation.

As a follow-on step, metal sulfide samples were produced ( $\mathrm{ZnS}, \mathrm{Ni}_{3} \mathrm{~S}_{4}, \mathrm{SnS}, \mathrm{CoS}_{2}, \mathrm{CdS}$, and $\mathrm{CuS}$ ) and were oxidized to produce $\mathrm{ZnO}, \mathrm{NiO}, \mathrm{SnO}_{2}, \mathrm{Co}_{3} \mathrm{O}_{4}$, and $\mathrm{CuO}$. A mixed sample strategy of three runs of 24 liter dual parallel reactor was subsequently followed. In order to initiate the 24 liter reaction, 12 liter of growth medium was added to two 13.25 liter glass carboys, equipped with ventilation ports to the headspace, which were autoclaved for 2 hours. The carboys were cooled to 70 
${ }^{\circ} \mathrm{C}$ in the autoclave and were further cooled with continuous purging using $\mathrm{N}_{2}$ gas through a $0.2-$ micron filter overnight and put into plastic containers which were connected to a $65{ }^{\circ} \mathrm{C}$ water bath. To produce $\mathrm{SnS}$, the solution was enriched for 24 hours using $10 \mathrm{mM}$ of glucose, $6 \mathrm{mM}$ thiosulfate as a sulfur source, and $2 \%$ mid-log growth phase stock solution. $\mathrm{SnCl}$ was dosed after 24 hours. The three runs showed high reproducibility of average crystallite size of 3.3-3.7 nm and of the yields ranged of 15.9-17.0g / 24 liters. Figure 1.36 shows the resultant samples from the three runs, while the analysis results are summarized in Table 1.10.

Table 1.10 SnS sample preparation (24 liter) of the three batches

\begin{tabular}{|c|c|c|c|c|}
\hline Run & $\begin{array}{c}\text { Condition (10 mM of } \\
\text { glucose, } 6 \mathrm{mM} \text { thiosulfate) }\end{array}$ & Sn & Yield & $\begin{array}{c}\text { Average } \\
\text { Crystallite } \\
\text { Size }\end{array}$ \\
\hline $1^{\text {st }}$, SNS140325A01 & 5 days in fresh medium & $4 \mathrm{mM}$ & $15.86 \mathrm{~g}$ & $3.3 \pm 0.2 \mathrm{~nm}$ \\
\hline $2^{\text {nd }}$, SNS140409A01 & 5 days in fresh medium & $4 \mathrm{mM}$ & $16.17 \mathrm{~g}$ & $3.5 \pm 0.4 \mathrm{~nm}$ \\
\hline $3^{\text {rd }}$, SNS140425A01 & 5 days in fresh medium & $4 \mathrm{mM}$ & $17.01 \mathrm{~g}$ & $3.7 \pm 0.2 \mathrm{~nm}$ \\
\hline
\end{tabular}

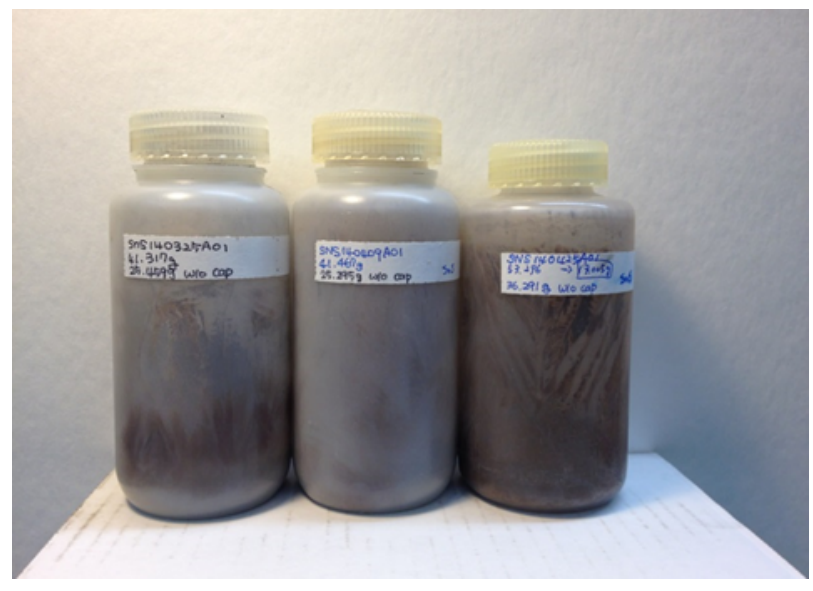

Figure 1.36. Samples produced from the low temperature materials synthesis project. The material was $>50 \mathrm{~g}$ of $\mathrm{SnS}$ and was oxidized to $\mathrm{n}$-type $\mathrm{SnO}_{2}$ and used for gas sensing.

The consecutive batch processing resulted in $\sim 200 \mathrm{~g}$ of $\mathrm{SnS}$, but we observed that the surface had an organic coating. We investigated the water quality, medium buffer, purging period, aging, and exposure to the air, and identified the main problem being the accumulation of carbon sources in the water. Using purified water solved this problem.

The nanofermented $\mathrm{SnS}$ nanoparticles were oxidized to produce $\mathrm{SnO}_{2}$ phase at an annealing temperature of $800^{\circ} \mathrm{C}$. The oxidation efficiency was found to strongly depend on the initial $\mathrm{SnS}$ amount during annealing. As was mentioned above for the case of $\mathrm{ZnO}$, it was possible to induce pure $\mathrm{ZnO}$ phase even at an annealing temperature of $600^{\circ} \mathrm{C}$ for $<1 \mathrm{~g} \mathrm{ZnS}$. In Table 1.11 we show the information for the $\mathrm{SnO}_{2}$ phases and the size of the nanocrystals. The resulting powders were analyzed using $\mathrm{x}$-ray diffraction to determine the phases formed during the heat treatment (see Figure 1.37). 
Table 1.11 Conversion of $\mathrm{SnS}$ nanoparticles to $\mathrm{SnO}_{2}$

\begin{tabular}{|c|c|c|c|c|c|c|c|}
\hline Sample & Phase & $\begin{array}{l}\text { Wt } \\
\%\end{array}$ & Space Group & $\mathrm{a}(\AA)$ & $\begin{array}{c}\mathrm{b}(\AA) \\
\beta\end{array}$ & $\begin{array}{c}\mathrm{c}(\AA) \\
\gamma\end{array}$ & $\begin{array}{l}\text { Size } \\
(\AA)\end{array}$ \\
\hline \multirow{2}{*}{$\begin{array}{l}\text { SnS } 130711 \mathrm{~A} 01 \\
800 \mathrm{C}, 5 \text { hours, } \\
500 \mathrm{ccm} \mathrm{O} \mathrm{O}_{2} \\
\mathrm{P} 10997\end{array}$} & \multirow{2}{*}{$\mathrm{SnO}_{2}$} & \multirow{2}{*}{100} & Tetragonal & 4.744 & 4.744 & 3.189 & \multirow{2}{*}{120} \\
\hline & & & $\mathrm{P} 42 / \mathrm{m} \mathrm{n} \mathrm{m} \mathrm{(136)}$ & 90 & 90 & 90 & \\
\hline \multirow{2}{*}{$\begin{array}{l}\text { SnS } 140409 \text { A01 } \\
800 \mathrm{C}, 5 \text { hours, } \\
500 \mathrm{ccm} \mathrm{O} \mathrm{O}_{2} \\
\mathrm{P} 10999\end{array}$} & \multirow{2}{*}{$\mathrm{SnO}_{2}$} & \multirow{2}{*}{100} & Tetragonal & 4.742 & 4.742 & 3.188 & \multirow{2}{*}{300} \\
\hline & & & $\mathrm{P} 42 / \mathrm{m} \mathrm{n} \mathrm{m} \mathrm{(136)}$ & 90 & 90 & 90 & \\
\hline \multirow{2}{*}{$\begin{array}{l}\text { SnS } 140325 \mathrm{~A} 01 \\
800 \mathrm{C} \text { for } 5 \text { hours, } \\
500 \mathrm{ccm} \mathrm{O}_{2} \\
\mathrm{P} 10998\end{array}$} & \multirow{2}{*}{$\mathrm{SnO}_{2}$} & \multirow{2}{*}{100} & Tetragonal & 4.737 & 4.737 & 3.185 & \multirow{2}{*}{460} \\
\hline & & & $\mathrm{P} 42 / \mathrm{m} \mathrm{n} \mathrm{m} \mathrm{(136)}$ & 90 & 90 & 90 & \\
\hline
\end{tabular}

The nanofermentation $\mathrm{Ni}_{3} \mathrm{~S}_{4}$ nanoparticles were oxidized to produce the $\mathrm{NiO}$ phase at an annealing temperature of $800^{\circ} \mathrm{C}$. The resulting powders were analyzed using $\mathrm{x}$-ray diffraction to determine the phases formed during the heat treatment (see Figure 1.38). The NiO nanoparticles were further processed to produce printable ink that was used to characterize electrical conductivity of $\mathrm{NiO} / \mathrm{Pt}$ resistors. The as-processed nanoparticles were found to be quite large, as shown in Figure 1.39, with average size exceeding $10 \mu \mathrm{m}$. The large size is possibly due to particle agglomeration, which calls for improved chemical processes for effective passivation of particle surface bonds.

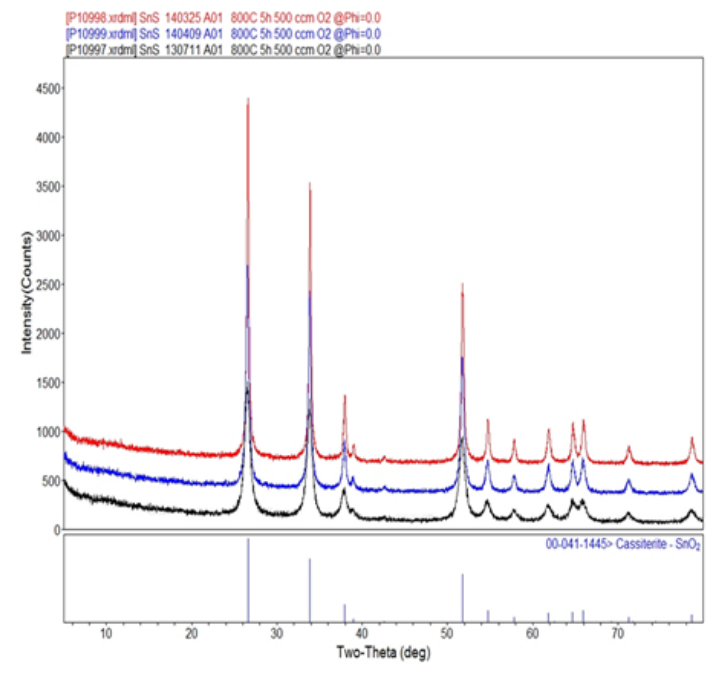

Figure 1.37. The $\mathrm{SnS}$ was converted to $\mathrm{SnO}_{2}$ with oxidation in $\mathrm{O}_{2}$ atmosphere at $800^{\circ} \mathrm{C}$ for 1 hour.

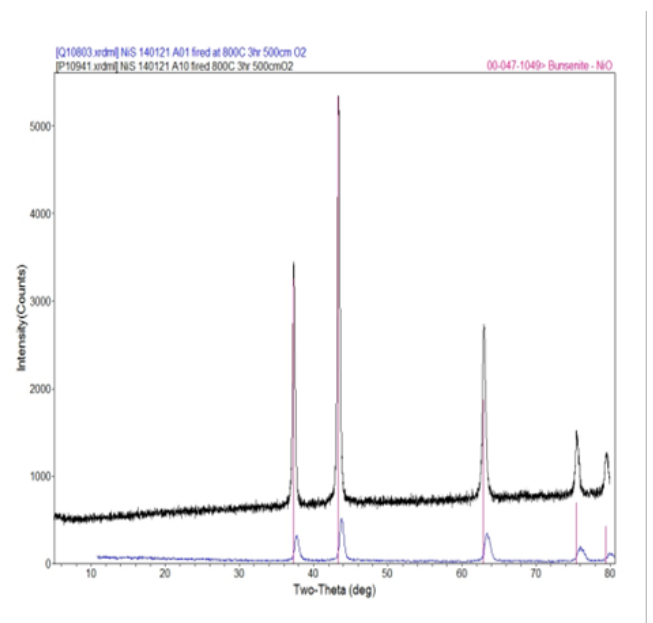

Figure 1.38. The Ni3S4 was converted to $\mathrm{NiO}$ with oxidation in $\mathrm{O} 2$ atmosphere at $800^{\circ} \mathrm{C}$ for 1 hour. A pure $\mathrm{NiO}$ phase was produced after annealing at $800^{\circ} \mathrm{C}$. 


\section{Particle Size Distribution}

After Milling for Ink Development

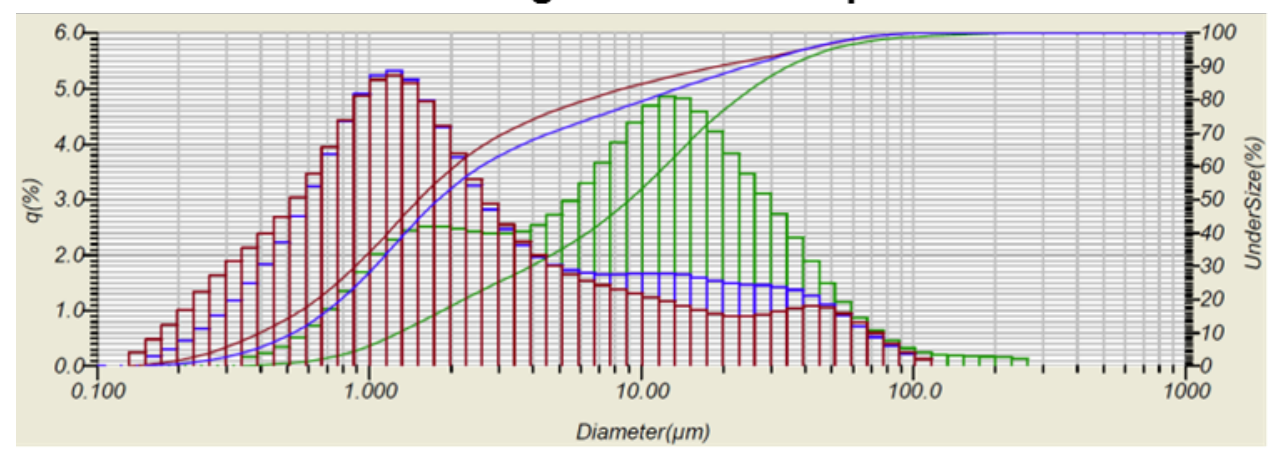

Figure 1.39. Particle size distribution of $\mathrm{NiO}$ nanoparticles that were processed to produce a printable ink.

\subsubsection{Sensor Test Apparatus}

An essential part of the sensor development was the design of the experimental environment where sensors can be tested. We teamed with the Center for Nanophase Materials Sciences and developed Environmental Chamber Vacuum- Referenced, Multimode Sensing system which allowed for cross referenced testing of thin film sensors in a controlled environment (see Figure 1.40). The pressure in the vacuum chamber was $10^{-6}$ Torr, the humidity $0-90 \%$, the oxygen concentration $0.01-20 \%$, the temperature $\mathrm{RT}<\mathrm{T}<85 \mathrm{C}$. The programmable multimode testing was with a Keithley 4200SC (PV, diode, transistor.), along with broad frequency impedance spectroscopy, transmittance, reflectance, photoluminescence, Raman (1064 nm) measurements. The system was also implemented for in situ measurements of changes in the thin film weight using a $5 \mathrm{MHz}$ Quartz crystal microbalance. Multimodality of sensors was also advanced with in situ statistical analysis (PCA, MCR).

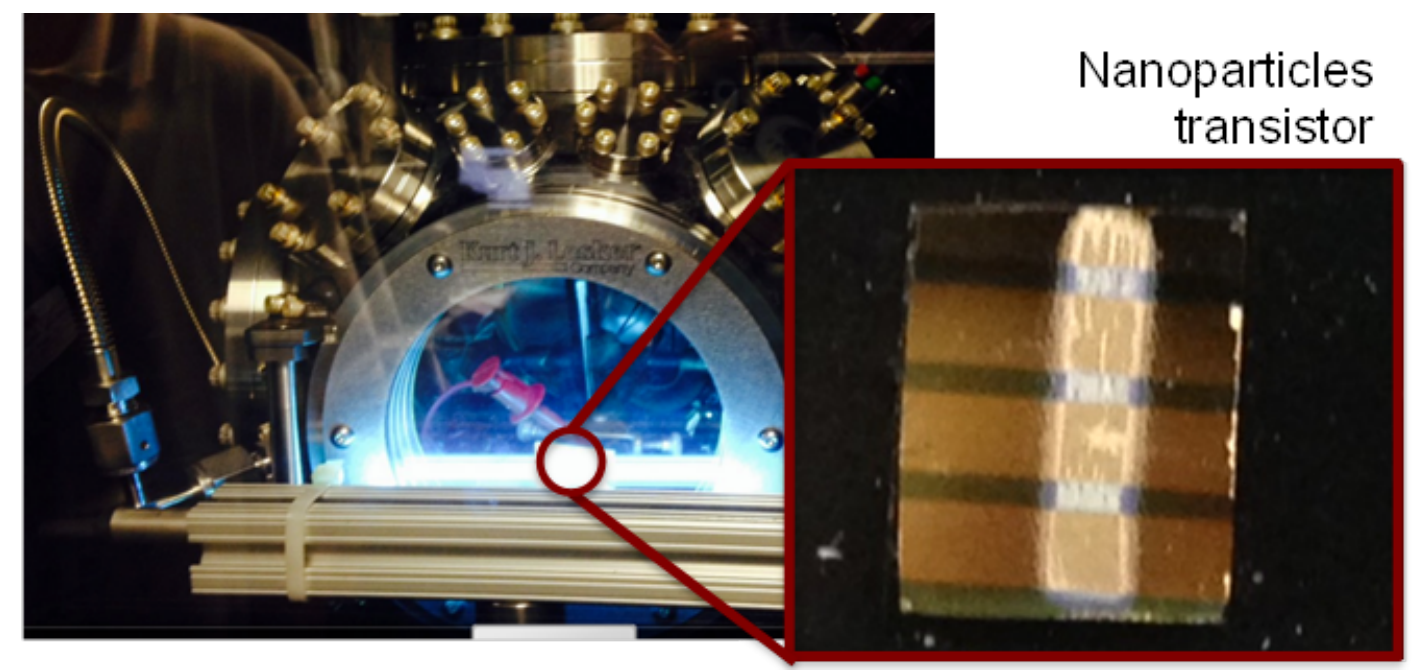

Figure 1.40. Background image of the Environmental Chamber. The inset shows the screen printed $\mathrm{ZnO}$ transistor on the surface of $\mathrm{Si} / \mathrm{SiO} 2$ wafer tested in the chamber. 


\subsubsection{Low Temperature Nickel Oxide Thin Films}

Metal oxide thin films offer a unique combination of structural, electrical, optical, magnetic, and chemical properties for advanced sensor applications. Low temperature processing of high quality metal oxide thin films was critical for integration into a flexible sensor platform. The investigation focused on the development of low temperature nickel oxide $(\mathrm{NiO})$ thin films exploiting $\mathrm{RF}$ sputtering and pulse thermal processing techniques. $\mathrm{NiO}$ is a wide bandgap $p$-type semiconducting material with suitable properties for gas sensor, battery, supercapacitor, organic light emitting diode (OLED), and solar cell applications. The electrochromic properties of $\mathrm{NiO}$ are being exploited for a wide range of applications, such as energy efficient windows and multi-color displays. The details of the $\mathrm{NiO}$ thin film deposition process and the impact of process temperature on the microstructural, optical, and electrical characteristics are highlighted in the following sections.

\subsubsection{Nickel Oxide Thin Film Deposition}

$\mathrm{NiO}$ thin films were deposited by a RF sputtering technique from a stoichiometric $\mathrm{NiO}$ target in an $\mathrm{Ar}+\mathrm{O}_{2}$ atmosphere. As shown in Figure 1.41(a), the $\mathrm{Ar} / \mathrm{O}_{2}$ ratio, chamber pressure, and RF sputtering power were kept constant at $80 \%, 9$ mTorr, and $350 \mathrm{~W}$, respectively, while the effects of process temperature on the thin film growth and properties were analyzed in the range of $25-400^{\circ} \mathrm{C}$. The $\mathrm{NiO}$ thin films were deposited on bare $\mathrm{Si}$ and fused quartz substrates. The deposition rate of $\mathrm{NiO}$ thin films, as shown in Figure 1.41(b), was found to decrease from $12.7 \AA / \mathrm{min}$ to $10.3 \AA / \mathrm{min}$ with an increase in process temperature from 25 to $400{ }^{\circ} \mathrm{C}$. The observed temperature dependence of the thin film deposition rate indicated that the grain growth was influenced by the process temperature.

\section{Process: RF Power Mode}

- Power: 350W

- Pressure: 9 mTorr (3-15 mTorr)

- $\mathrm{T}=25-400^{\circ} \mathrm{C}$

- $\mathrm{Ar} / \mathrm{O}_{2}$ ratio: $80 / 20$

(a)

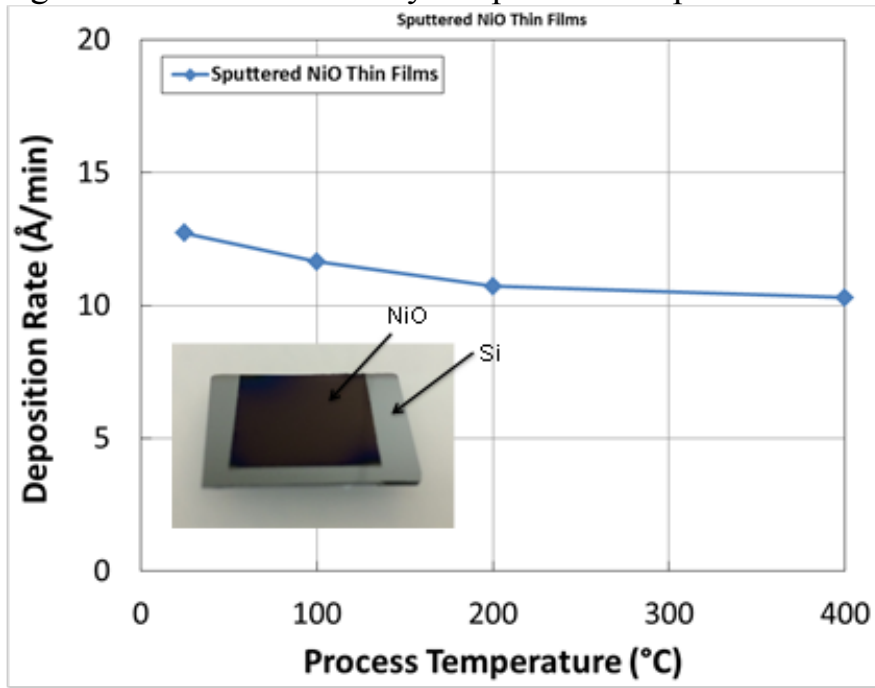

(b)

Figure 1.41. (a) Process conditions and (b) deposition rate of rf sputtered NiO thin films as a function of substrate temperature.

\subsubsection{Nickel Oxide Microstructure}

The crystallinity of $\mathrm{NiO}$ thin films was analyzed by x-ray diffraction (XRD) technique. Figure 1.42 shows the XRD patterns of the films deposited on bare Si and fused quartz substrates. All the films deposited in the temperature range of $25-400^{\circ} \mathrm{C}$ exhibited a polycrystalline cubic phase. The XRD peak intensity was found to increase with an increase in deposition temperature indicating an improvement in film crystallinity. The XRD patterns did not show any appreciable change in the FCC phase with process temperature indicating the formation of a stable phase. The $\mathrm{NiO}$ thin films 
showed a preferential growth along the [111] direction. A similar XRD pattern was obtained on films deposited on amorphous quartz substrates indicating that the film growth was not influenced by substrate microstructure. The morphology of the films was analyzed by scanning electron microcopy as shown in Figure 1.43.
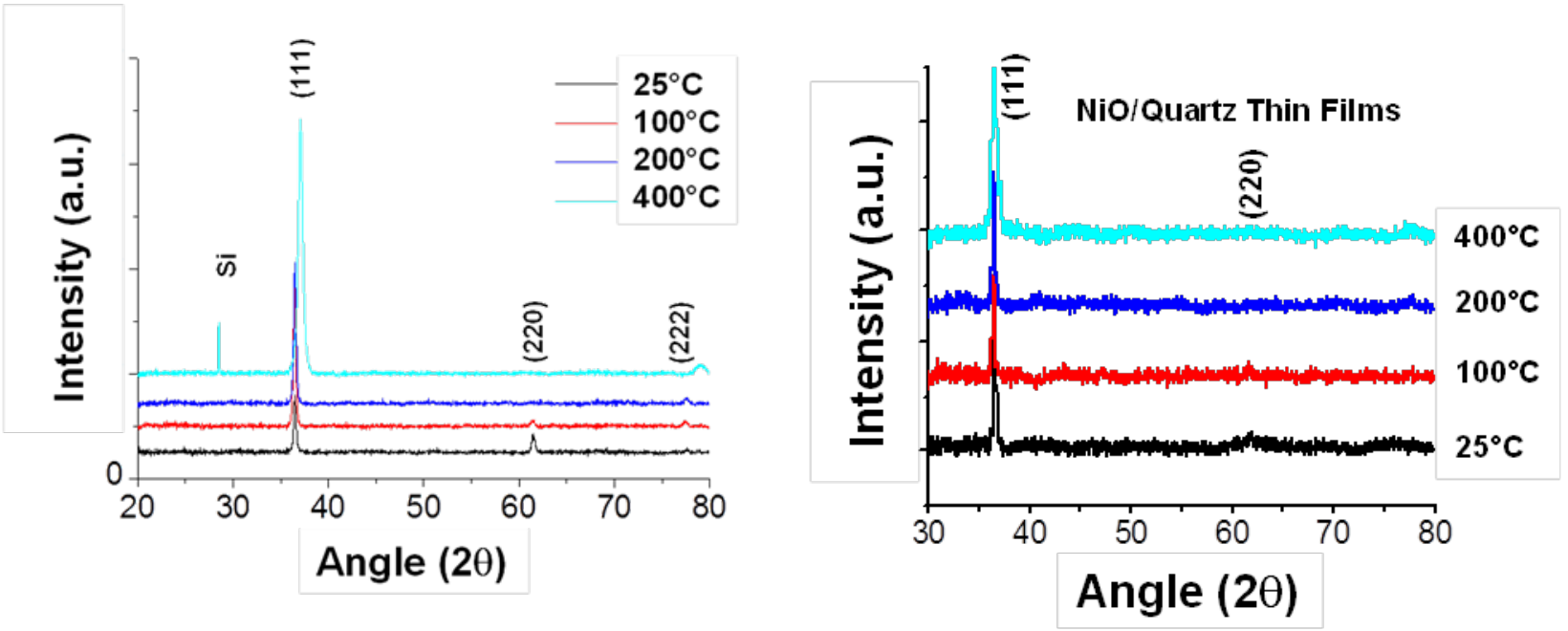

Figure 1.42. The x-ray diffraction patterns of NiO thin films deposited on (a) Si and (b) quartz substrates.
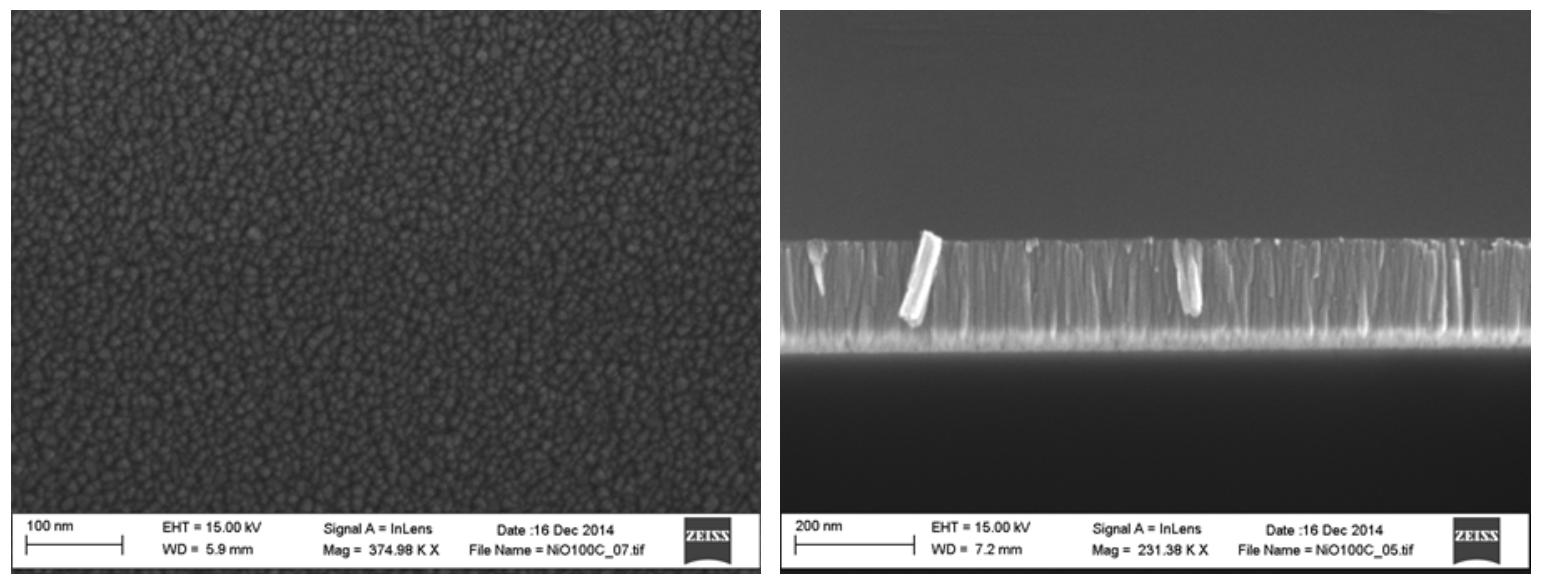

Figure 1.43. (a) Surface morphology and (b) SEM cross section of NiO thin films deposited on Si substrates at a temperature of $100^{\circ} \mathrm{C}$.

The surface and cross-sectional morphology of $\mathrm{NiO}$ films deposited at $100^{\circ} \mathrm{C}$ showed a dense microstructure with fine grains. The SEM cross section showed closely packed columnar grains which grew perpendicular to the film surface. The high crystallinity and dense microstructure of $\mathrm{NiO}$ thin films deposited at low process temperatures show promise for integration on flexible paper and plastic substrates.

\subsubsection{Nickel Oxide Optical Properties}

The optical properties of $\mathrm{NiO}$ thin films were established from the transmittance and reflectance measurements (Figure 1.44) conducted at room temperature using a UV-VIS-NIR spectrophotometer. The optical transmittance of the NiO thin films, as shown in Figure 1.44(a), indicated a wide bandgap in the process temperature range of $25-400{ }^{\circ} \mathrm{C}$ with absorption edge in the UV part of the spectrum. 
The optical transmittance of $\mathrm{NiO}$ thin films did not show any appreciable temperature dependence down to a process temperature of $100^{\circ} \mathrm{C}$ while it was found to decrease appreciably at $25^{\circ} \mathrm{C}$. To gain further insight into the optical response, the optical absorption coefficient, $\alpha$, was calculated from the optical transmittance, $T$, and reflectance, $R$, data using the relation: $\alpha=1 / t\left[\ln T /\left(1-R^{2}\right)\right]$, where $t$ is the film thickness.
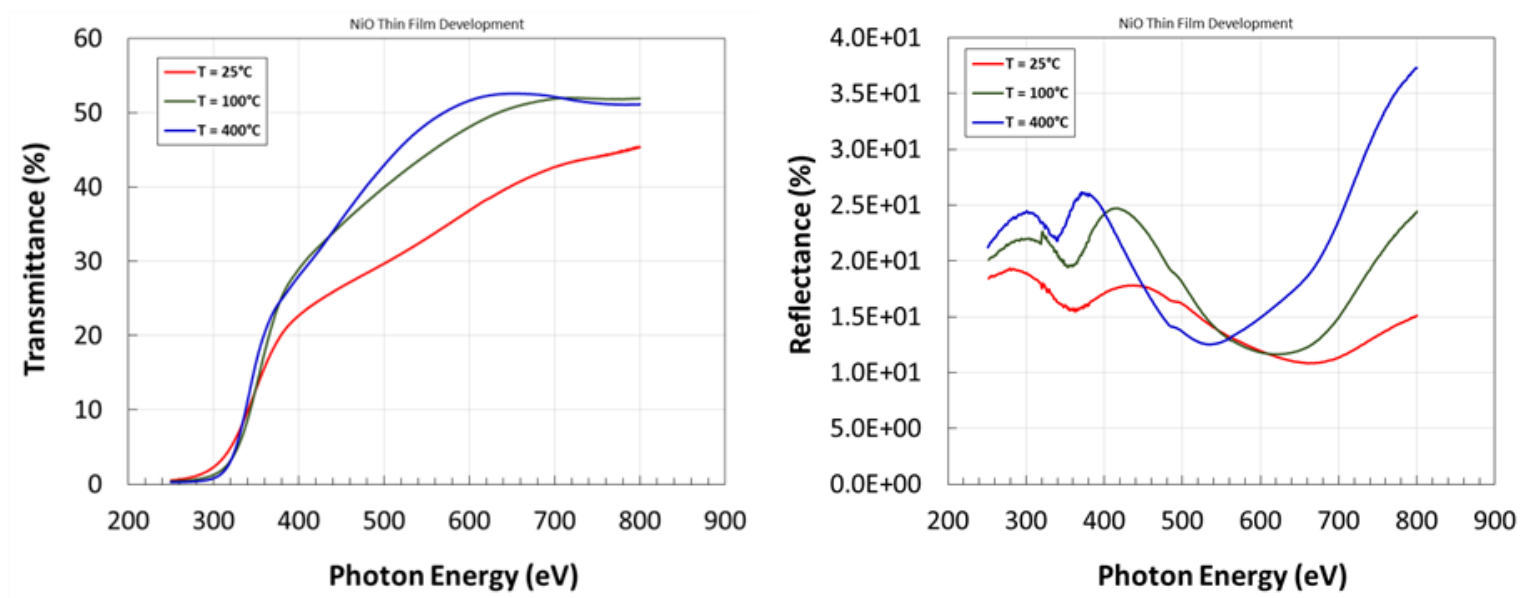

Figure 1.44. (a) Optical transmittance and (b) reflectance of $\mathrm{NiO}$ thin films as a function of process temperature.

For direct bandgap $\mathrm{NiO}$ thin films, the absorption coefficient dependence on the incident photon energy, $h v$, is given by the relation: $\alpha h v=A\left(h v-E_{g}\right)^{1 / 2}$, where $E g$ is the material bandgap. The energy of the optical bandgap of $\mathrm{NiO}$ thin films was established by extrapolating the linear part of the $(\alpha h v)^{2} v s h v$ plot to abscissa. The optical bandgap of the NiO thin films, as depicted in Figure 1.45, did not show a strong dependence on the process temperature and the value remained higher than 3.3 $\mathrm{eV}$ in the process temperature range of $25-400^{\circ} \mathrm{C}$. The observed wide bandgap value even at a process temperature of $25^{\circ} \mathrm{C}$ is in good agreement with the values reported for films processed at higher temperatures exceeding $200{ }^{\circ} \mathrm{C}$, indicating high optical density of the present films. The present $\mathrm{NiO}$ thin films were deposited at an oxygen partial pressure of $20 \%$. The high optical bandgap of $\mathrm{NiO}$ thin films deposited at $25^{\circ} \mathrm{C}$ indicates that the film stoichiometry and density were dictated by the oxygen partial pressure and the plasma density rather than the thermal state of the substrate. The observed optical properties of $\mathrm{NiO}$ thin films are consistent with the XRD and SEM analyses indicating the formation of a well-defined polycrystalline phase and a dense microstructure even at a low process temperature of $25^{\circ} \mathrm{C}$. 


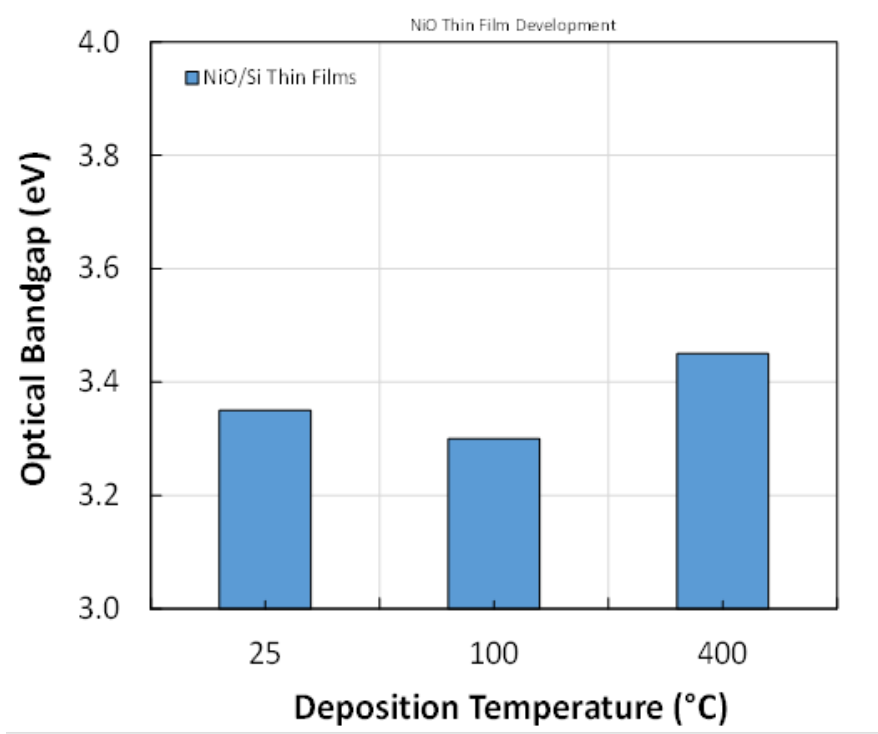

Figure 1.45. The optical bandgap of $\mathrm{NiO}$ thin films as a function of process temperature.

The $n$ - $k$ optical dispersion characteristics were established from the spectroscopic ellipsometry measurements as shown in Figure 1.45( $\mathrm{a}$ and $\mathrm{b}$ ). Spectroscopic ellipsometry data were obtained using a two-modulator generalized ellipsometer (2-MGE) at an angle of incidence of $65.01^{\circ}$. $\mathrm{NiO}$ thin films were modeled as a simple three layer structure (interface- $1 / \mathrm{NiO} /$ interface-2) to fit the TaucLorentz dispersion function. Interface-1 was modeled through Bruggeman effective medium approximation, consisting of $50 \%$ void and $50 \%$ of the $\mathrm{NiO}$ medium below. Interface- 2 was modeled as native oxide layer on the $\mathrm{Si}$ substrate. The optical constant of the $\mathrm{NiO}$ thin films were wellbehaved, as shown in Figure 1.46, and the energy dispersion matched well with the reported data on single crystal, thin films, and nanoparticles. The observed results are consistent with the XRD findings showing a well-define crystalline FCC phase in NiO thin films even at a low processing temperature of $25^{\circ} \mathrm{C}$.
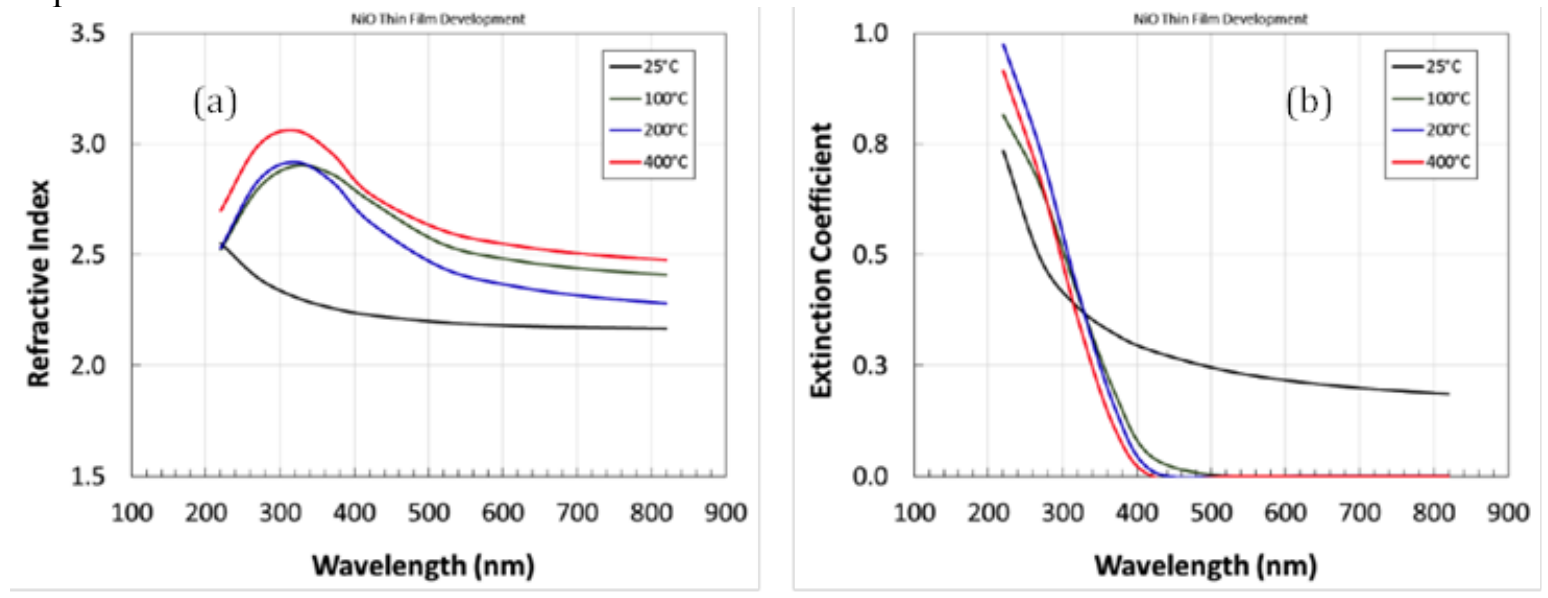

Figure 1.46. (a) Refractive index and (b) extinction coefficient of $\mathrm{NiO}$ thin films as a function of process temperature.

\subsubsection{Nickel Oxide Hall Measurements}

The electrical properties of the $\mathrm{NiO}$ thin films deposited on quartz substrates were evaluated by Hall effect measurements. As shown in Figure 1.47a, the carrier concentration values were found to be 
positive in the entire processing temperature range of $25-400{ }^{\circ} \mathrm{C}$ indicating $p$-type conduction. The mobility of the $\mathrm{NiO}$ films was measured to be $0.40 \mathrm{~cm}^{2} / \mathrm{Vs}$ at a process temperature of $400{ }^{\circ} \mathrm{C}$. As shown in Figure 1.47 (b), the mobility value decreased with a decrease in process temperature down to $100{ }^{\circ} \mathrm{C}$. However, it was found to be increase appreciably to a value of $0.86 \mathrm{~cm}^{2} / \mathrm{Vs}$ at a process temperature of $25^{\circ} \mathrm{C}$. The observed increase clearly indicates a change in the thin film microstructure that influences carrier scattering. The XRD patterns did not indicate any significant change in the film crystallinity as a function of process temperature. Detailed electrical measurements will provide further insight into the critical process parameters dictating the thin film growth and observed high mobility at a process temperature of $25^{\circ} \mathrm{C}$. The $p$-type $\mathrm{NiO}$ films showed a high carrier concentration of about $4.2 \times 10^{17} \mathrm{~cm}^{-3}$ at a process temperature of $25^{\circ} \mathrm{C}$. The carrier concentration remained in the range of $(4.2-22.4) \times 10^{17} \mathrm{~cm}^{-3}$ for films processed in the temperature range of $25-400{ }^{\circ} \mathrm{C}$ indicating that the film density was well maintained even at low process temperatures suitable for flexible substrate integration.
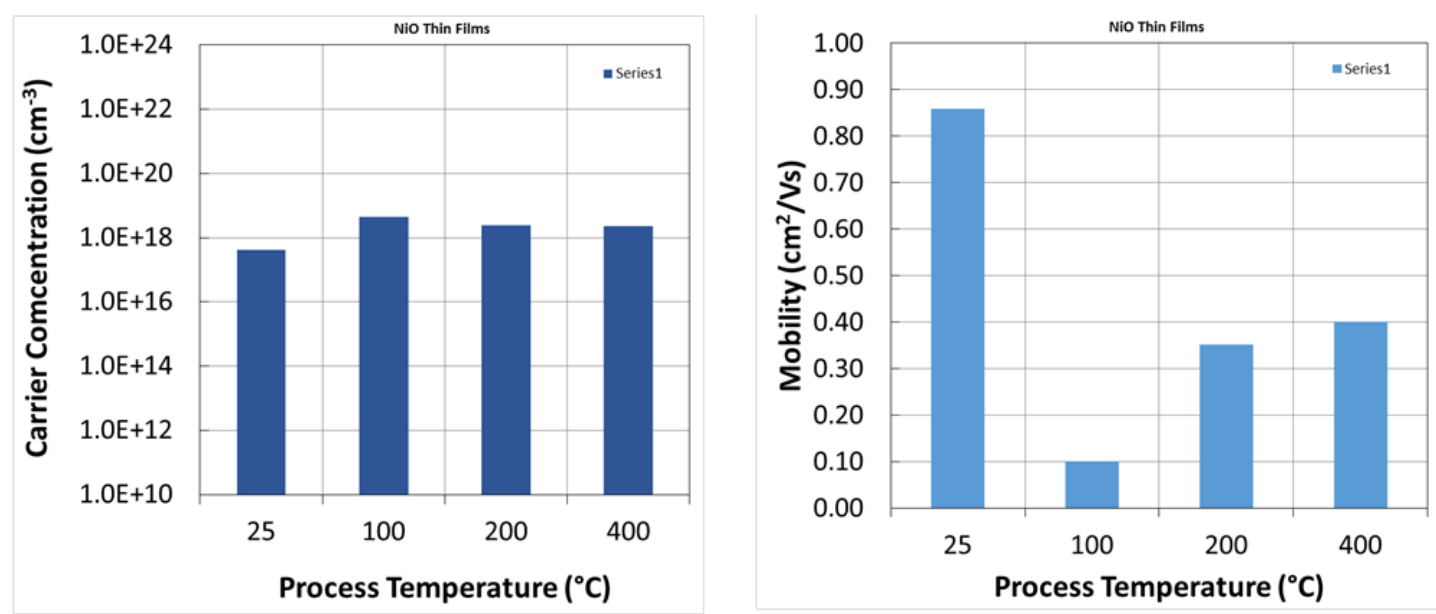

Figure 1.47. (a) Carrier concentration and (b) Hall mobility of $\mathrm{NiO}$ thin films as a function of process temperatures.

\subsubsection{NiO Low Temperature Photonic Curing}

The pulse thermal processing (PTP) technique was explored to influence $\mathrm{NiO}$ thin film properties at low thermal budgets suitable for integration on low temperature substrates such as plastic and paper. The photonic curing system is capable of delivering a high intensity, short duration $(<20 \mathrm{~ms})$ broadband light (200-1400nm) from a directed xenon flash lamp to generate very high processing temperatures required for thin film densification, recrystallization and annealing, but without damaging the underlying temperature-sensitive substrates or co-integrated electronics. The photonic curing system is capable of delivering maximum peak power in excess of $20 \mathrm{~kW} / \mathrm{cm}^{2}$ while the pulse width can be maintained as short as 30 microseconds. Table 1.12 lists the sample splits for the first lot of samples used in PTP investigation. The peak pulse power was varied in the range of 2.6-9.1 $\mathrm{kW} / \mathrm{cm}^{2}$ while keeping the pulse width and number of pulses constant at $500 \mu$ s and 25 , respectively. A test sample exposed to 100 pulses of $1 \mathrm{~ms}$ duration was also included in the first lot to analyze the impact of thermal budget on thin film microstructure. Figure 1.48 shows the impact of PTP curing on the crystallinity of $\mathrm{NiO}$ thin films deposited at process temperatures of 25 and $100{ }^{\circ} \mathrm{C}$. The crystallinity of the films deposited at $25^{\circ} \mathrm{C}$ did not show any significant impact of the photonic curing while the films deposited at $100{ }^{\circ} \mathrm{C}$ showed a small variation in the [220] peak intensity while the dominant [111] peak intensity did not change appreciably. The SEM, optical and electrical measurements need to be conducted in details to gain further insight into the impact of photonic 
curing on film microstructure, optical absorption characteristics, and electrical conductivity. Overall, the high quality of the low temperature deposited $\mathrm{NiO}$ thin films in combination with the low thermal budget photonic curing show promise for the integration of $\mathrm{NiO}$ sensor on a flexible sensor platform.

Table 1.12 Samples splits for low thermal budget photonic curing investigation

\begin{tabular}{|c|c|c|c|c|}
\hline S.No. & Sample ID & $\begin{array}{c}\text { Dep. Temp. } \\
\left({ }^{\circ} \mathrm{C}\right)\end{array}$ & $\begin{array}{c}\text { Peak Power } \\
\left(\mathrm{kW} / \mathrm{cm}^{2}\right)\end{array}$ & Number of Pulses \\
\hline 1 & NiO_RT & 25 & \multirow[t]{2}{*}{2.6} & \multirow[t]{2}{*}{25 pulses } \\
\hline 2 & NiO_100 & 100 & & \\
\hline 3 & NiO_RT & 25 & \multirow[t]{2}{*}{4.3} & \multirow[t]{2}{*}{25 pulses } \\
\hline 4 & NiO_100 & 100 & & \\
\hline 5 & NiO_RT & 25 & \multirow[t]{2}{*}{6.4} & \multirow[t]{2}{*}{25 pulses } \\
\hline 6 & NiO_100 & 100 & & \\
\hline 7 & NiO_RT & 25 & \multirow[t]{2}{*}{9.1} & \multirow[t]{2}{*}{25 pulses } \\
\hline 8 & NiO_100 & 100 & & \\
\hline 9 & NiO_RT & 25 & \multirow[t]{2}{*}{2.4} & \multirow[t]{2}{*}{100 pulses } \\
\hline 10 & NiO_100 & 100 & & \\
\hline 11 & RT_Ref & 25 & No PTP & Reference sample \\
\hline 12 & 100_Ref & 100 & No PTP & Reference sample \\
\hline
\end{tabular}

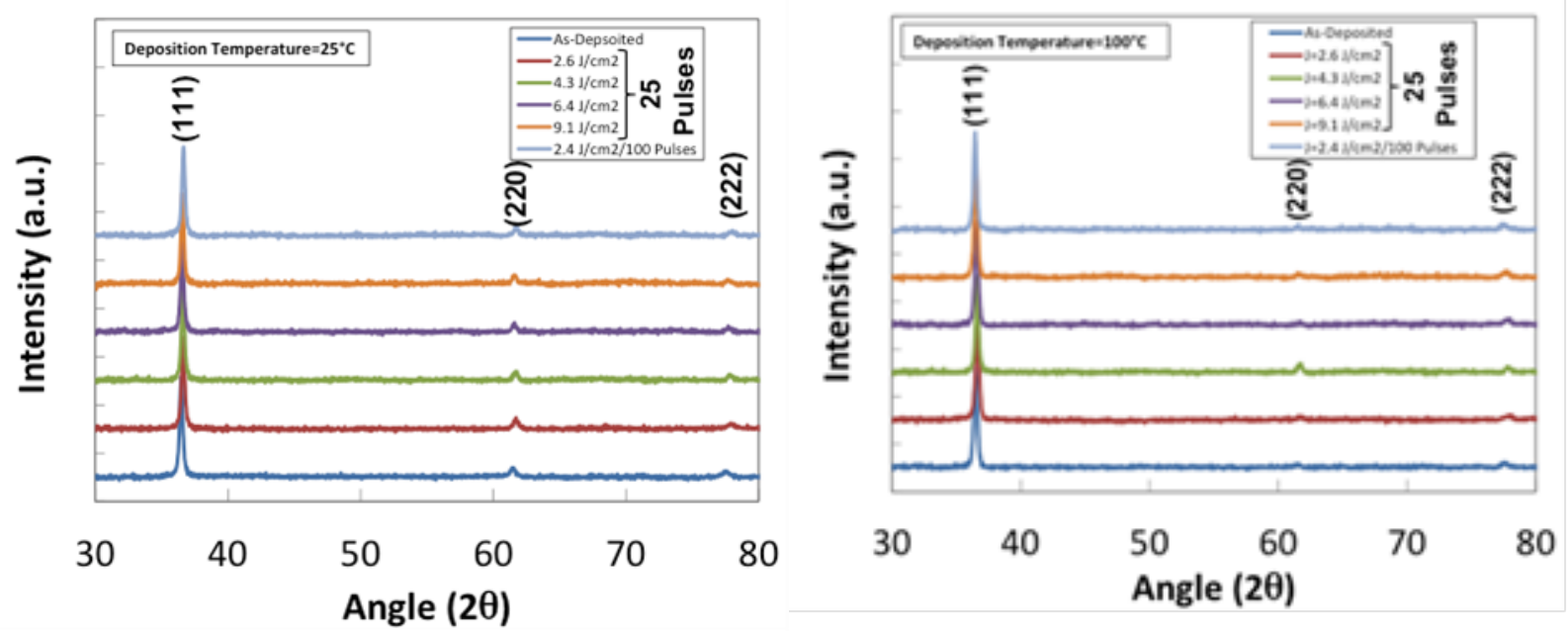

Figure 1.48. The impact of photonic curing on the structural characteristics of $\mathrm{NiO}$ thin films deposited at $25^{\circ} \mathrm{C}$ and $100{ }^{\circ} \mathrm{C}$. 


\subsection{THIN FILM DEPOSITION}

Thin films were deposited by ultrasonic spray deposition technique (see later in section below). Pulse thermal processing (PTP) techniques were used for low thermal budget annealing of thin films and the impact of PTP treatment was evaluated by performing thin film characterization in terms of the structural, optical, and electrical properties.

\subsubsection{Ultrasonic Spray Coating Technique}

The nanoparticle thin films were deposited by ultrasonic spray deposition technique using sono-spray system as shown in Figure 1.49. The robotic sono-spray system offers partial atmosphere and process temperature controls $\left(25-180^{\circ} \mathrm{C}\right)$ for high quality thin films development. The key features of the sono-spray systems are highlighted below:

- Processing nanoparticle dispersions into thin film

- Optimization of single/multi-layer coatings on quartz and glass slides

- Characterization of optical properties of thin film assemblies

- Optimization of temperature, dispersant, particle size, surface treatment conditions

\subsubsection{Optical Characterization}

Two different optical techniques were used to characterize $\mathrm{ZnS}$ and $\mathrm{CdS}$ nanoparticles fabricated using nanofermentation: 1) diffuse reflectance and 2) photoluminescence.

\subsubsection{Diffuse Reflectance}

Diffuse reflectance measurements were taken on samples of nanoparticles enclosed in a mini-cuvette $\sim 200$ microns thick. The measurement apparatus consisted of a Perkin-Elmer Lambda 900 spectrophotometer equipped with an integrating sphere, pictured in Figure 1.49. The resulting diffuse reflectance data, shown in Figure 1.50(a) can be interpreted using the Kubelka-Munk theory ${ }^{1}$, originally developed for the characterization of paper. This theory works best for optically thick materials, where very little of the light is transmitted through the sample and when the amount of reflected light is $>\sim 20 \%$. Using this theory, the ratio of the absorption coefficient $\mathrm{S}$ and the scattering coefficient $\mathrm{S}$ can be expressed in terms of the reflectance $\mathrm{R}$ as:

$$
\frac{\alpha}{S}=\frac{(1-R)^{2}}{2 R}
$$

The resulting values of the ratio $\alpha / \mathrm{S}$ are shown in Figure 1.50(b).

1 Kubelka and Munk, Zeit. Fuer Tekn. Physik, 12, 593 (1931) 

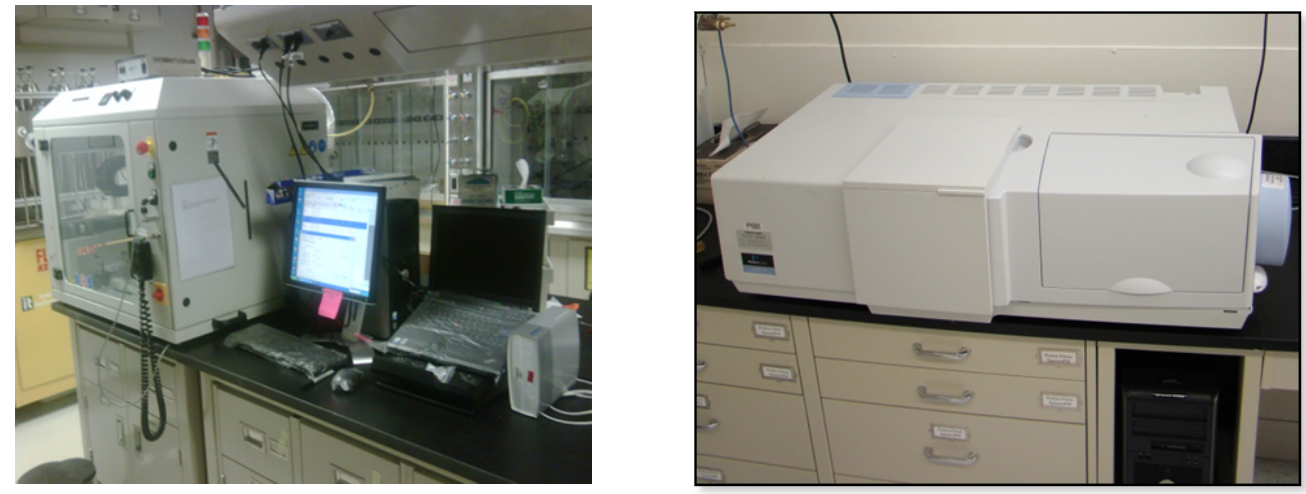

Figure 1.49. Sono-spray deposition system for nanoparticle thin film processing (L) and Perkin-EImer Lambda 900 spectrophotometer with integrating sphere attachment (R)
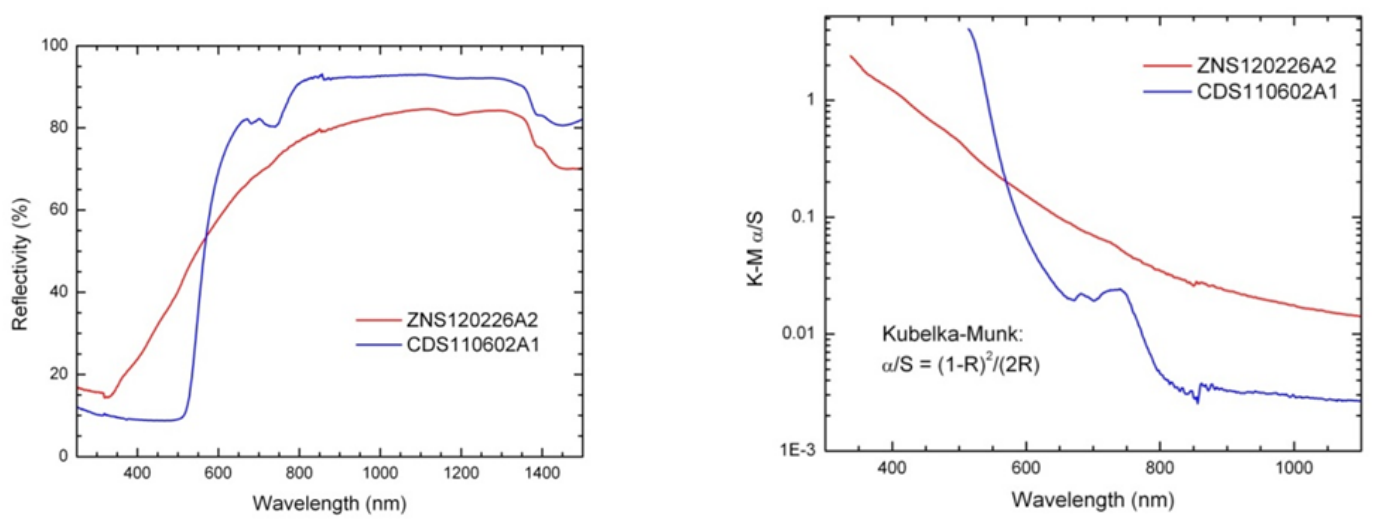

Figure 1.50. a) The measured diffuse reflectivity from samples of nanocrystalline $\mathrm{ZnS}$ and $\mathrm{CdS}$. b) the values of the Kubelka-Munk $\alpha / S$ coefficient, determined from the reflectivity measurements.

From the data shown in Figure 1.50(b), we can conclude that the onset of optical absorption in the CdS nanoparticles is quite sharp, and the band edge is $\sim 500 \mathrm{~nm}$. This is consistent with the observed band edge of thin-film CdS $(\sim 2.5 \mathrm{eV} \sim 500 \mathrm{~nm})^{2}$, commonly used as emitters for CdTe and CIGS solar cells. Moreover, the CdS spectrum in Figure 1.50(b) shows two absorption features near 670 and $720 \mathrm{~nm}$. The identity of these features is not known at this time.

While the onset of optical absorption for CdS is quite abrupt, the onset of optical absorption for $\mathrm{ZnS}$ is quite gradual. It is not known why this is the case, but may relate to the size distribution of the $\mathrm{ZnS}$ nanoparticles in the sample.

\subsubsection{Photoluminescence}

The photoluminescence system consists of several light sources and two detectors. The sample can

2 J. Li, J. Chen, and R. W. Collins, Appl. Phys. Lett. 97, 181909 (2010). 
be illuminated using 1) a pulsed nitrogen laser $(337 \mathrm{~nm}), 2)$ a multi-line argon-ion laser with 6 lines between 457 and $514 \mathrm{~nm}$, and a HeNe laser at $633 \mathrm{~nm}$. The light can be detected using a photomultiplier tube $(200-850 \mathrm{~nm})$ or a cooled Ge detector $(600-1700 \mathrm{~nm})$. The monochromator is an Oriel/Newport Cornerstone 260 with 3 gratings and a filter wheel. The sample can be placed in a closed cycle cryostat to obtain temperatures from $10 \mathrm{~K}$ to $400 \mathrm{~K}$. The detected light can be continuous wave $(\mathrm{CW})$ or chopped, where the chopped system incorporates a Stanford Research 830 lock-in amplifier.

Figure 1.51 shows photoluminescence spectra from the $\mathrm{CdS}$ and $\mathrm{ZnS}$ samples, also discussed above. The ZnS sample shows a large, broad PL peak near $520 \mathrm{~nm}$, which is not duplicated with the CdS sample. This broad PL peak in $\mathrm{ZnS}$ is similar to that observed in other nanocrystalline samples of $\mathrm{ZnS}^{3}$, where the peak and breadth of the observed PL is a function of the distribution of particle size in the sample. The spectra from both samples show several distinct lines, where the line width is determined by the instrumental line width ( $1.5 \mathrm{~nm}$ for the present configuration). The origin of these distinct lines is unknown at present.

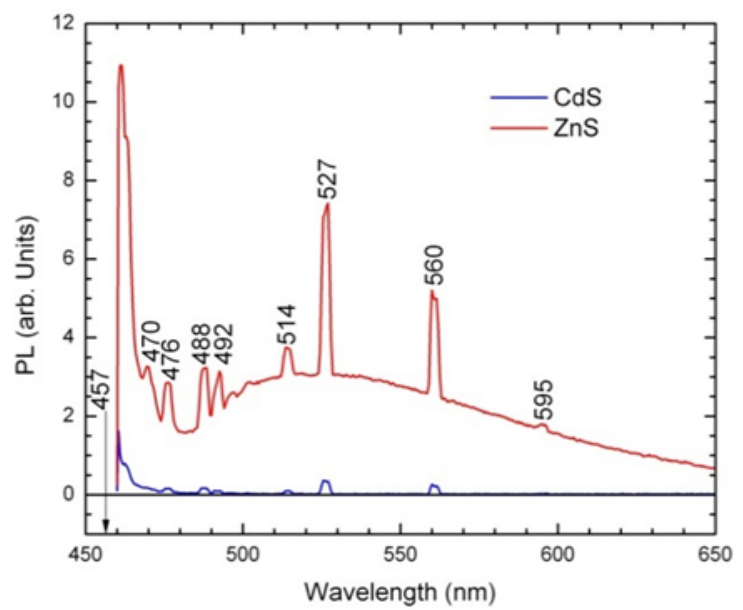

Figure 1.51. The photoluminescence spectra from the CdS and ZnS nanocrystalline samples.

\subsubsection{Sonospray Coatings}

The sonospray deposition system was shown to be advantageous over other deposition approaches, including jet printing, to generate high quality nanoparticle coatings. The Exactacoat setup was used for thin deposition on the surface of $2 \times 3$ inches glass slides. Prior to depositions, glass slides were cleaned in isopropanol and acetone for $15 \mathrm{~min}$ under mild sonication. The thermodynamics of $\mathrm{ZnS}$ PEI solution on PE and glass substrates was tested using dynamic sessile drop method for a series of solutions of water, PEI and ZnS in PEI. The results are summarized in Figure 1.52.

As shown in Figure 1.52(a), the introduction of PEI increases contact angle from 13 to 33 degrees for glass. Nanoparticles further increase the value of contact angle solution to 34 degrees. While further increase in $\mathrm{ZnS}$ nanoparticles concentration may further increase the contact angle, it is still smaller that contact angle of water-PEI-ZnS solutions on plastic. This suggests that the glass substrate is better for sonospray deposition of $\mathrm{ZnS}-\mathrm{PEI}$ solution.

3 C. Unni, D. Philip, and K. G. Gopchandran, Opt. Mat. 32, 169 (2009). 

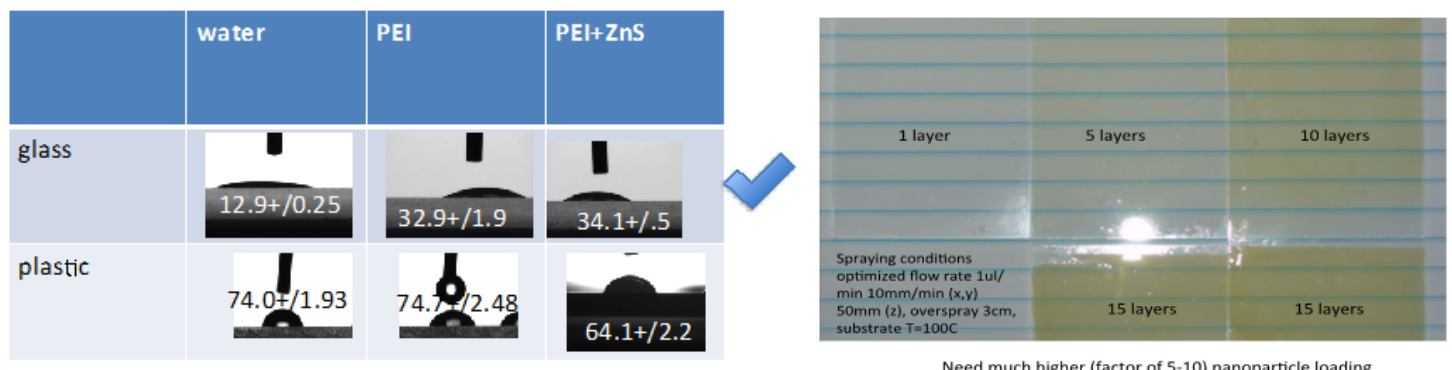

Need much higher (factor of 5-10) nanoparticle loading

Figure 1.52. The ZnS thin film coating development in terms of (a) solvent/substrate interaction and (b) nanoparticle loading effect analysis.

\subsubsection{Thin Film Characterization}

We tested nanoparticle deposition in various regimes of the flow rate, $\mathrm{X}-\mathrm{Y}$ raster speed and Z- high. The substrate temperature was varied from room temperature to $100 \mathrm{oC}$. The optimal deposition was obtained for substrates heated to $100 \mathrm{oC}$. It allowed complete removal of water after each pass. Multilayer depositions were tested as well. Figure 1.52(b) shows a series of depositions of 1, 5, 10 and 15 layers of PEI-ZnS solution using optimized deposition conditions: flow rate $1 \mu \mathrm{l} / \mathrm{min}$ $10 \mathrm{~mm} / \mathrm{min}(\mathrm{x}, \mathrm{y}) 50 \mathrm{~mm}(\mathrm{z})$, overspray $3 \mathrm{~cm}$, substrate $\mathrm{T}=100^{\circ} \mathrm{C}$. All films demonstrate good coating uniformity, which was tested using optical transmittance measurements in different areas of the film (Figure 1.52(b)).

After deposition the coatings were further dried in the air and tested using LED $450 \mathrm{~nm}$ transmittance/photoluminescence. The results and photograph of transmittance through 15 layers system are shown in Figure 1.53. The nanoparticle film uniformity was found to be independent of the number of coatings.

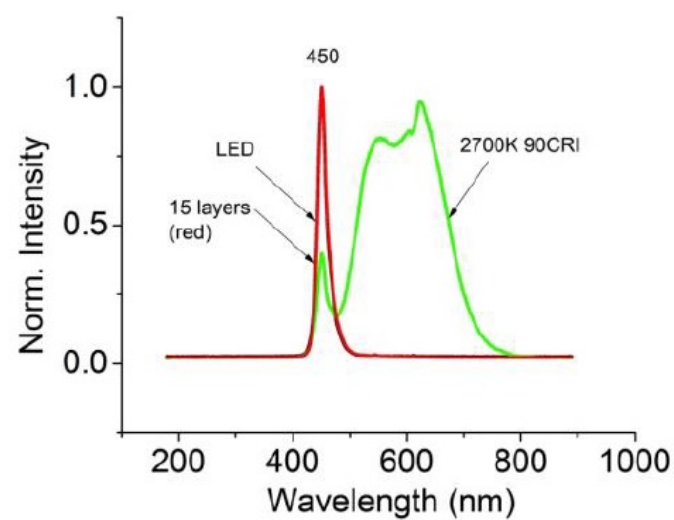

(a)

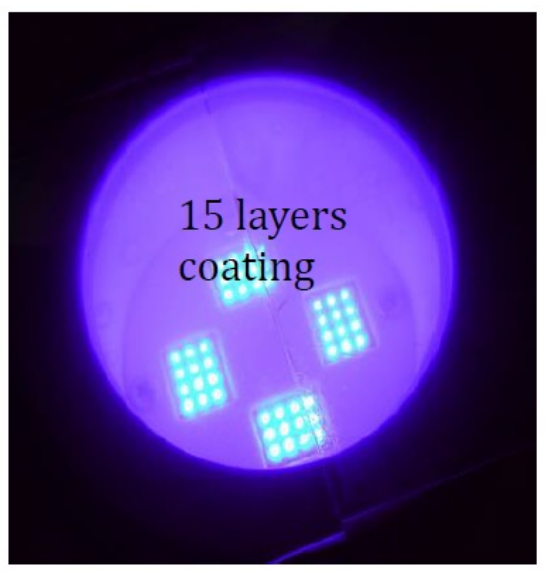

(b)

Figure 1.53. (a) Optical transmittance and (b) thin film uniformity as analyzed using 450nm LED. 
Preliminary experiments on PEI removal indicated that $200^{\circ} \mathrm{C}$ annealing was not sufficient for complete removal of organic binder. After 40 min annealing, the color of PEI-ZnS film turned brown and did not change after further annealing at the same temperature for 3 hours. Thermal gravimetric analysis of 600, 1200 and $1800 \mathrm{MW}$ PEI; as shown in Figure 1.54, suggests that a high temperature is required to remove PEI from the coating. The requirement to anneal nanoparticle coatings is temperatures $>600^{\circ} \mathrm{C}$ for complete PEI removal. Low temperature annealing $\left(200^{\circ} \mathrm{C}\right)$ is expected to leave as much as $98 \%$ and as low as $92 \%$ PEI on the surface. One has to consider the consequence of using high temperature annealing for binder removal as it may alter chemical composition of the nanoparticles.

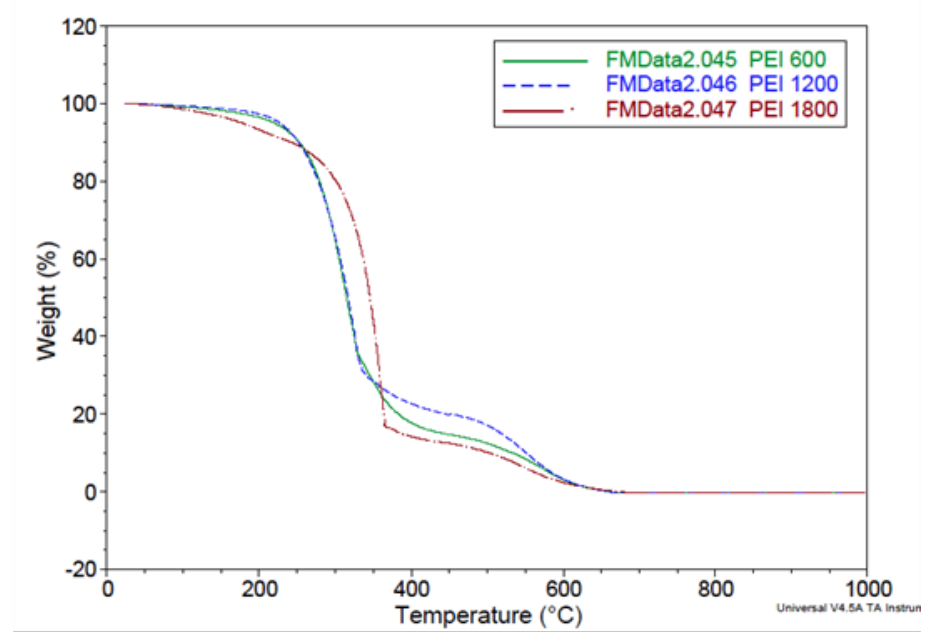

Figure 1.54. Thermal gravimetric analysis of PEI based ZnS nanoparticle inks.

The main conclusions are as follows:

- Small contact angles for (ZnS-PEI 600) glass surface were measured

- Good quality coatings (up to 15 layer coating was sono-sprayed) on glass were made

- Viscosity of water- PEI solutions is adequate for sonospray deposition of nanoparticles

- Annealing at $200^{\circ} \mathrm{C}$ in air does not remove PEI completely

- Temperatures higher than $600 \mathrm{C}$ in air are needed to remove PEI coating.

- Higher $(5 \mathrm{x}) \mathrm{ZnS}$ loadings are needed to create high optical density of phosphor.

In the next phase of the study, the films were annealed at various temperatures to remove organic components, density microstructure, and improve crystallinity. Figure 1.55 shows the optical images of the $\mathrm{ZnS}$ nanoparticle $(\sim 3.8 \mathrm{~nm})$ films before and after annealing at $800^{\circ} \mathrm{C}$. There was no visible difference in the surface microstructure. The film adhesion was a major challenge at this stage in the thin film development. The films were found to peel-off near the edges after annealing. The ink chemistry was being modified in close correlation with the film adhesion characteristics to address the issue. 


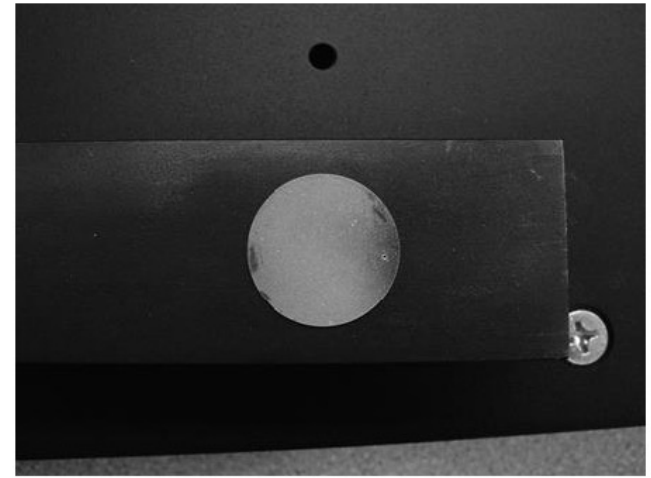

(a)

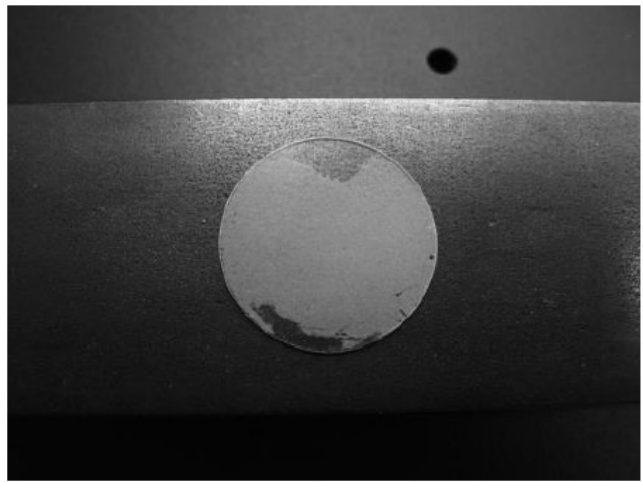

(b)

Figure 1.55. Optical images of the $\mathrm{ZnS}$ thin films deposited quartz substrates: (a) as-deposited, (b) annealed at $800^{\circ} \mathrm{C} / 30$ minutes.

A high resolution optical microscope was used to further analyze the surface morphology of the $\mathrm{ZnS}$ nanoparticle thin films. As shown in Figure 1.56, the films showed a significantly improved surface roughness profile and increased density after annealing. However, micro-cracks were also observed after annealing indicating a large change in film stress and density after annealing.

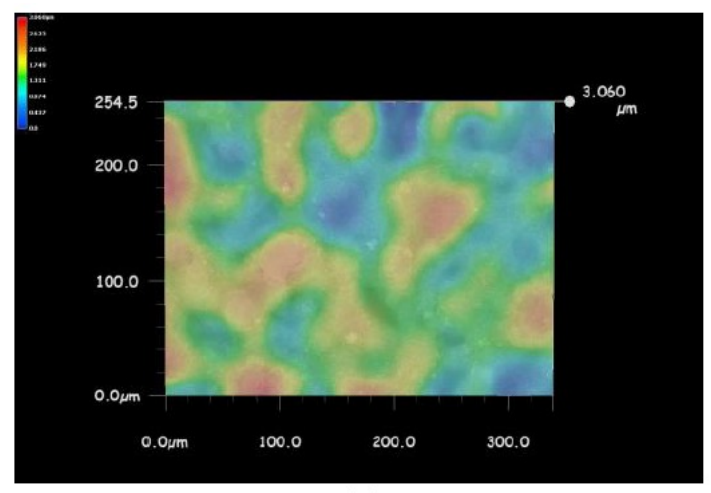

(a)

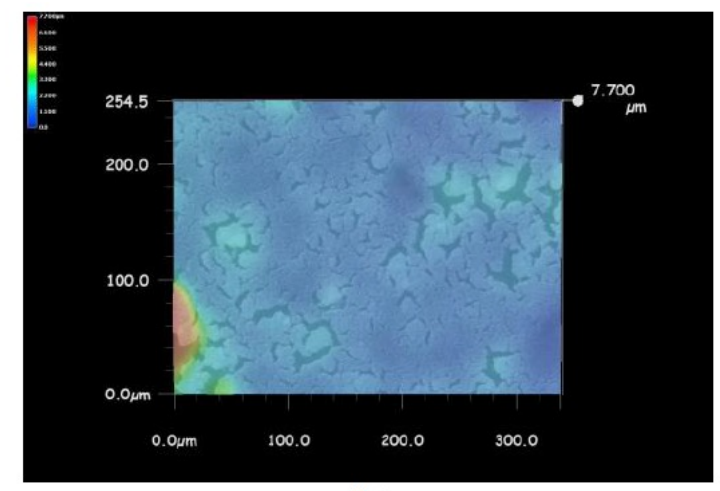

(b)

Figure 1.56. Surface morphology of $\mathrm{ZnS}$ nanoparticle thin films: (a) as-deposited, (b) annealed at $800^{\circ} \mathrm{C} / 30$ minutes.

Figure 1.57 shows the diffuse transmittance of the as-deposited and annealed $\mathrm{ZnS}$ thin films. The film transmittance was found to increase after annealing indicating an effective removal of the organic components. The optical absorption edge also shifted to higher energy levels indicating an improvement in $\mathrm{ZnS}$ crystallinity and microstructure. 


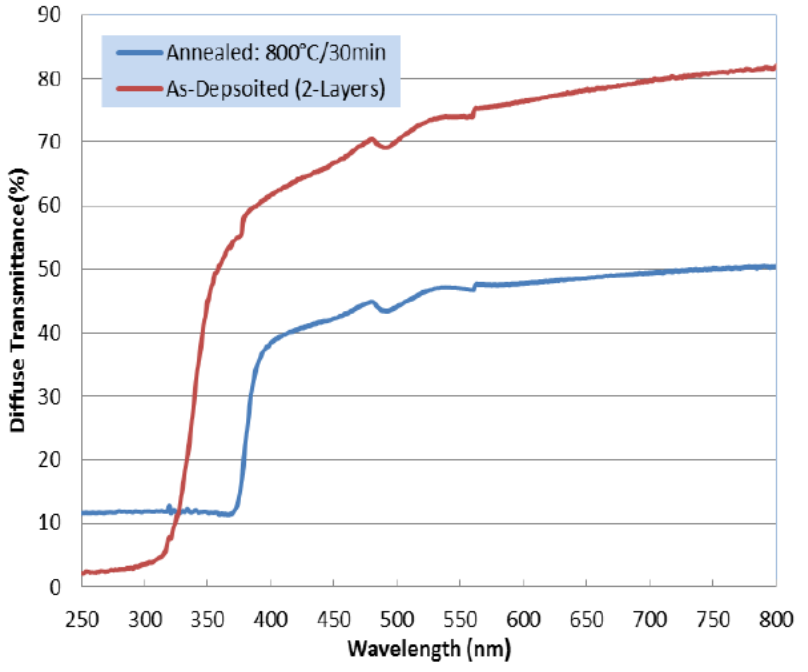

Figure 1.57. Diffused transmittance of $\mathrm{ZnS}$ nano-particle thin films deposited on quartz substrates.

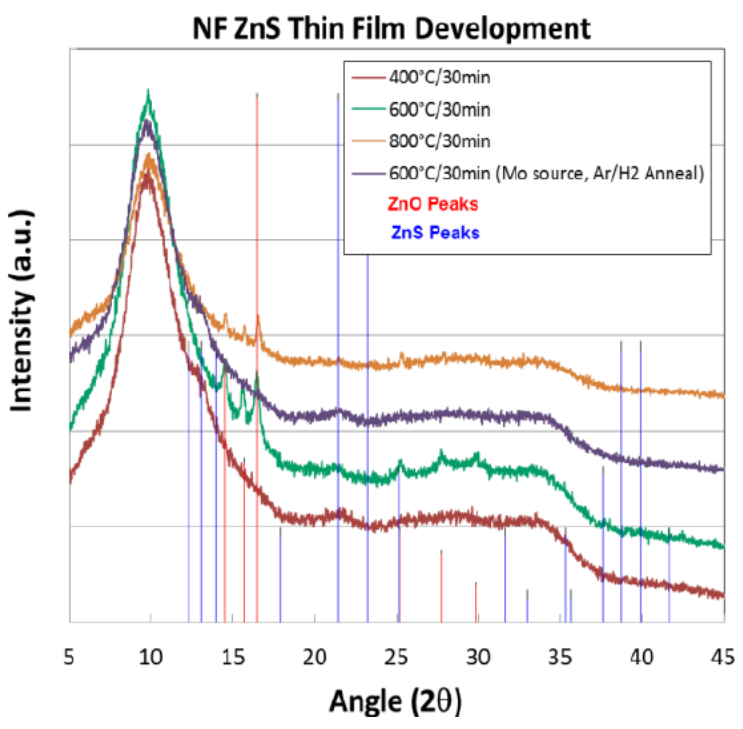

Figure 1.58. X-ray diffraction patterns of $\mathrm{ZnS}$ nanoparticle thin films as a function of annealing temperature

The crystalline nature of $\mathrm{ZnS}$ films was analyzed by $\mathrm{x}$-ray diffraction (XRD) technique as shown in Figure 1.58. The XRD measurements were conducted on $\mathrm{ZnS}$ nanoparticle $(\sim 3.8-4.7 \mathrm{~nm})$ thin films deposited on quartz substrates. The films were annealed in normal air and forming gas $\left(\mathrm{Ar} / \mathrm{H}_{2}(4 \%)\right)$ atmospheres. The annealing temperature had a strong influence on the $\mathrm{ZnO}$ phase formation. These results indicated that normal atmospheric air was enough to induce thin film oxidation even at a temperature of $400^{\circ} \mathrm{C}$. It is not clear if the ambient oxygen alone was the source of oxidation.

It was possible to prevent the oxidation of $\mathrm{ZnS}$ nanoparticle thin films in an $\mathrm{Ar} / \mathrm{H}_{2}(4 \%)$ atmosphere as shown in Figure 1.58. However, the films turned black even at a low $\mathrm{H}_{2}$ concentration of $4 \%$, indicating possible reduction of $\mathrm{ZnS}$ thin films.

Fourier transform infrared (FTIR) measurements were conducted to analyze the impact of annealing temperature on the impurity related bonds in $\mathrm{ZnS}$ thin films. The FTIR spectra of ZnS nanoparticle thin films is shown in Figure 1.59(a) while the organic activity in various $\mathrm{ZnS}$ samples processed by NanoFermentation (NF) approach is shown in Figure 1.59(b). The ZnS thin films showed a significant reduction in - $\mathrm{OH}, \mathrm{CH}_{3}, \mathrm{CH}_{2}$, and $\mathrm{COO}$ - bond concentration with increasing annealing temperature. Organic activity was very week after annealing at $800^{\circ} \mathrm{C}$. Overall, it was clear that annealing temperature has a strong influence on film crystallinity, microstructure, and optical properties. 

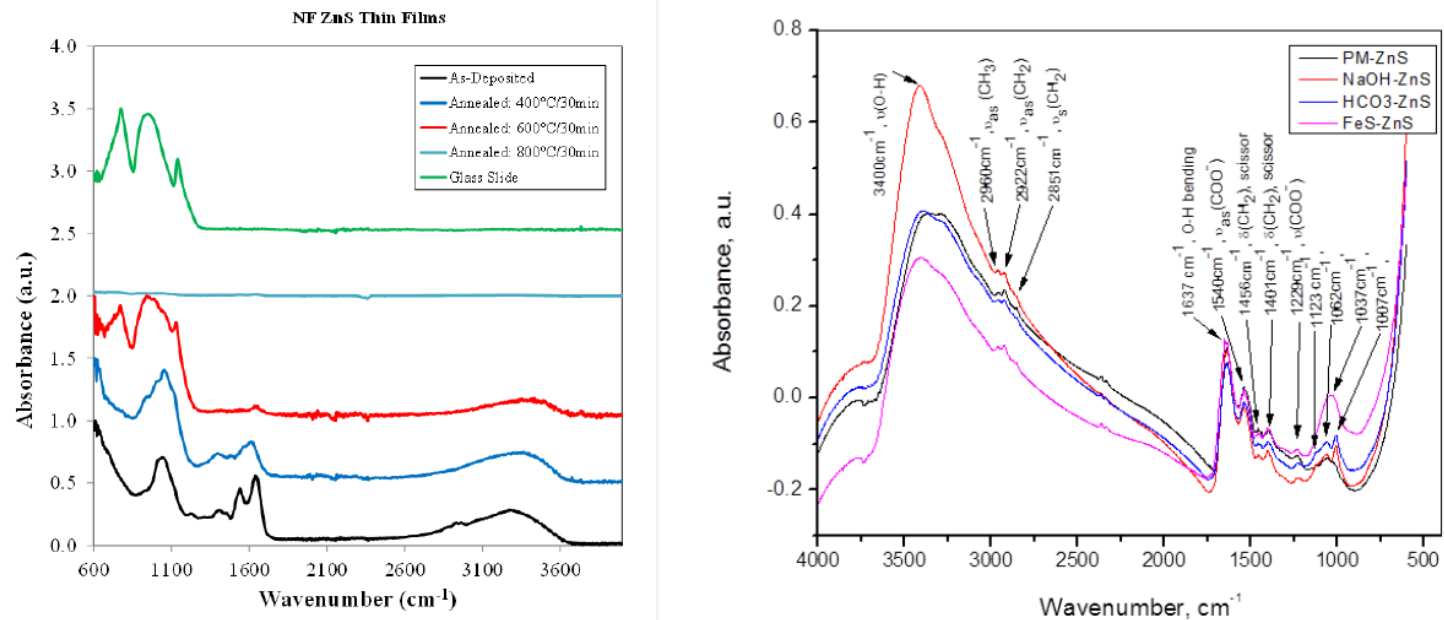

Figure 1.59. The FTIR spectra of $(\mathrm{L}) \mathrm{ZnS}$ thin films and $(\mathrm{R})$ various NF $\mathrm{ZnS}$ nanoparticle samples.

\subsubsection{Photoluminescence}

A HeCd laser, which emits 2 laser wavelengths - $325 \mathrm{~nm}$ and $442 \mathrm{~nm}(3.82$ and $2.81 \mathrm{eV})$ was used as an excitation source for the study of $\mathrm{ZnS}$ and $\mathrm{ZnO}$ materials (with band gaps of $\sim 3.54$ and $3.44 \mathrm{eV}$, respectively), since the $325 \mathrm{~nm}$ line has sufficient energy to promote electrons from the valence band to the conduction band. The previous cw excitation source was an argon ion laser, which emits 6 lines between 458 and $514 \mathrm{~nm}(2.71$ to $2.41 \mathrm{eV})$ all of which do not have sufficient photonic energy.

Several samples of nanoparticles of $\mathrm{ZnS}$ made by NanoFermentation were examined using photoluminescence, and the results are shown in Figure 1.60. The NanoFermentation process produced nanoparticles of various sizes, depending on the length of incubation in the solution with the bacteria. However, this process produced a distribution of particle sizes which was quite broad. Table 1.13 summarizes the growth conditions of the samples examined using photoluminescence, as well as identifying the plots shown in Figure 1.60. The blue data points in Figure 1.60 represent data from crystalline $\mathrm{ZnO}$, used as a reference. The scale of the $\mathrm{ZnO}$ PL spectrum is $\sim 50$ times that of the $\mathrm{PL}$ from the NanoFermentation samples. (That is, the $\mathrm{ZnO}$ signal is $\sim 50$ times stronger).

Table 1.13 Data for photoluminescence of nanoparticles in solution for Figure 1.60

\begin{tabular}{|l|l|l|l|}
\hline Graph color & Fabrication date & Peak Energy & $\begin{array}{l}\text { Est. average } \\
\text { particle size } \\
\text { (nm) }\end{array}$ \\
\hline Cyan & Sept 26 2012 & $2.89 \pm 0.02$ & $9.5 \pm 0.2$ \\
\hline Green & Aug. 23, 2012 & $2.86 \pm 0.02$ & $7 \pm 1$ \\
\hline Red & June 15, 2012 & $2.84 \pm 0.02$ & $2-3$ \\
\hline Magenta & June 27, 2012 & $2.83 \pm 0.02$ & $2-3$ \\
\hline $\begin{array}{l}\text { Blue (c- } \\
\text { ZnO) }\end{array}$ & --- & 3.28 & --- \\
\hline
\end{tabular}




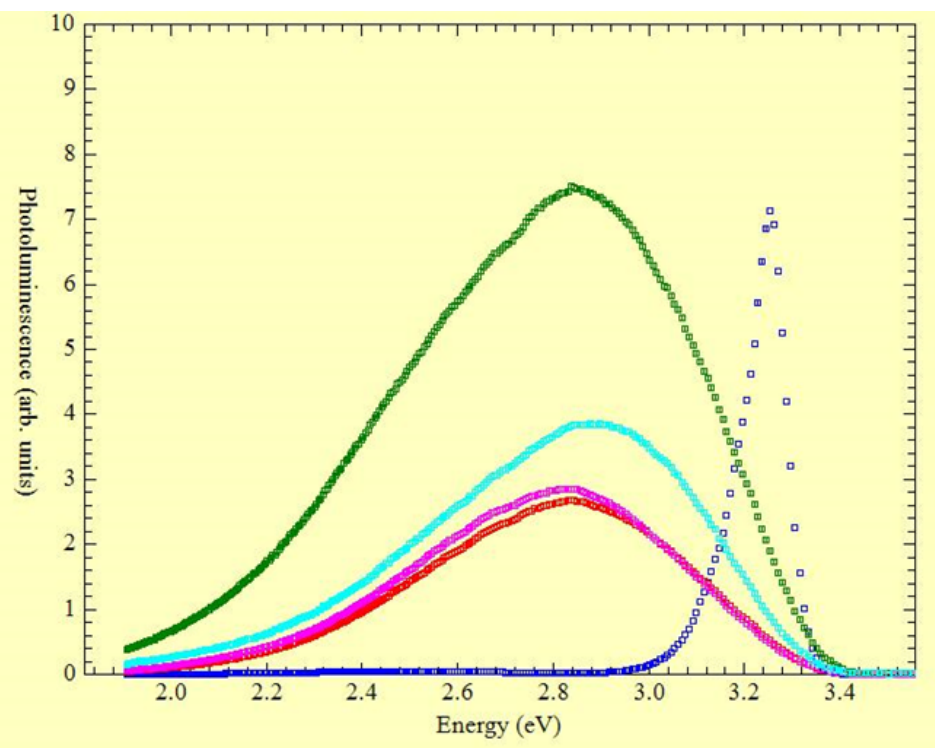

Figure 1.60. Photoluminescence of various sizes of nanoparticles in solution.

The PL from the $\mathrm{ZnO}$ sample showed the characteristic excitonic response just below the band edge. In addition, the $\mathrm{ZnO} P \mathrm{PL}$ shows a weak defect $\mathrm{PL} \sim 2.5 \mathrm{eV}$ (See Figure 1.61), related to defects in the crystalline material. While the shape of the PL spectra from the nanoparticle solutions is representative of the samples, the magnitude of the various spectra is not significant, since the data were taken at various sensitivities on the lock-in amplifier, and some of the samples (red and magenta) were significantly diluted. However, the PL shown in Figure 1.60 and Table 1.13 do show a very broad peak that does correlate with the estimated average particle size, with the higher peak PL energies corresponding to larger estimated average particle sizes. Moreover, the observed PL response is very broad.

While there was a correlation of peak PL energy with particle size, it was small and opposite to what is often observed with PL of nanoparticles (reduced particle size often results in an increase of PL energy). This, coupled with the observation that the PL spectra were very broad indicated that the PL measurement is integration over all particle sizes in the sample, and may not correlate well with the estimated average particle size.

Figure 1.61 shows the resulting PL spectra from a variety of consolidated films of $\mathrm{ZnS}$ nanoparticles, as well as a reference PL from a $\mathrm{ZnO}$ sample. The plot in Figure 1.61 is semi-logarithmic, and shows clearly that several samples show more than one peak. In particular, the defect-related peak in $\mathrm{ZnO}$, faintly observable in Figure 1.60, is obvious in Figure 1.61. 


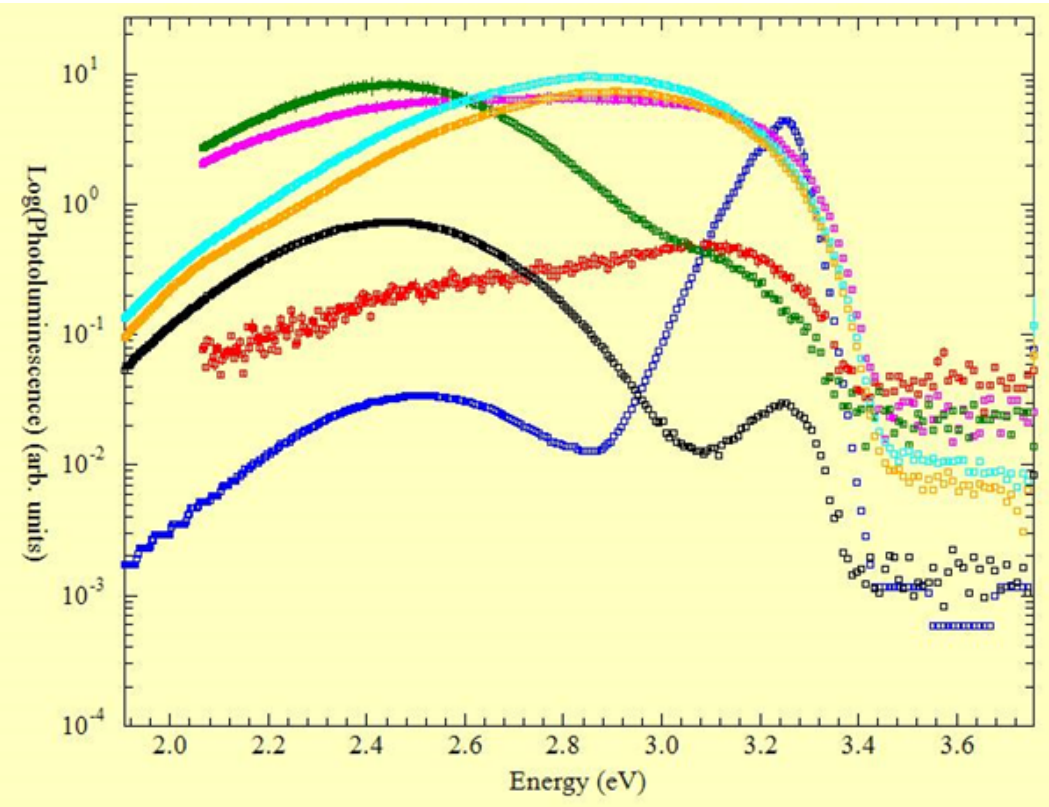

Figure 1.61. Photoluminescence of consolidated nanoparticle thin films.

The two sonosprayed samples starting with 15\% solution (Cyan and Orange) show very similar PL spectra that start just below the band gap of $\mathrm{ZnO}$ and are very broad. The single layer sample (Black) shows a broad PL peak near $2.5 \mathrm{eV}$, similar to that seen in the crystalline $\mathrm{ZnO}$, but with an additional peak near $3.28 \mathrm{eV}$, reminiscent of the excitonic peak of $\mathrm{ZnO}$.

Photoluminescence (PL) is clearly a very powerful tool for the examination of $\mathrm{ZnS}$ materials made from NanoFermentation nanoparticles. From solutions of NanoFermentation nanoparticles, we found that there was a correlation with the PL spectra and the estimated particle size, but the correlation was weak and contrary to the expected behavior; this is probably because our samples consisted of a wide distribution of particle sizes, as well as some other materials. Consolidated films of $\mathrm{ZnS}$ material show quite different PL spectra, depending on fabrication and post-fabrication annealing. When the samples were treated in air, there was evidence of $\mathrm{ZnO}$ being present, as well as other below-bandgap defects.

As a next step, $\mathrm{ZnS}$ particles were annealed at different temperatures in inert and $\mathrm{O}_{2}$ atmosphere to gain an understanding of the phase formation. The impact of the processing temperature on $\mathrm{ZnS}$ particle crystallinity was analyzed by x-ray diffraction (XRD) technique. The PL response of NF ZnS material was measured on discrete powder samples, and thin films deposited on quartz and alumina substrates. The photoemission performance of the nanoparticles was evaluated in terms of PL quantum efficiency. Table 1.14 lists the information on key samples that were used in the present study. 
Table 1.14 NF ZnS samples annealed at various conditions

\begin{tabular}{|c|c|c|c|c|}
\hline Label & Sample ID & Particle Size & Substrate & $\begin{array}{c}\text { Annealing Investigation } \\
\text { (Ar atmosphere) }\end{array}$ \\
\hline \multirow[t]{3}{*}{$120615 A 01$} & A & $3.1 \mathrm{~nm}$ & Quartz & \\
\hline & B & $3.1 \mathrm{~nm}$ & Quartz & $400^{\circ} \mathrm{C} / 30 \mathrm{~min}$ \\
\hline & $C$ & $3.1 \mathrm{~nm}$ & Quartz & $600^{\circ} / 30 \mathrm{~min}$ \\
\hline \multirow[t]{3}{*}{$120823 A 01$} & $\mathrm{~A}$ & $7.4 \mathrm{~nm}$ & Quartz & \\
\hline & B & $7.4 \mathrm{~nm}$ & Quartz & $400^{\circ} \mathrm{C} / 30 \mathrm{~min}$ \\
\hline & C & $7.4 \mathrm{~nm}$ & Quartz & $600^{\circ} / 30 \mathrm{~min}$ \\
\hline \multirow[t]{3}{*}{$120926 \mathrm{~A} 01$} & A & $9.5 \mathrm{~nm}$ & Quartz & \\
\hline & $B$ & $9.5 \mathrm{~nm}$ & Quartz & $400^{\circ} \mathrm{C} / 30 \mathrm{~min}$ \\
\hline & $C$ & $9.5 \mathrm{~nm}$ & Quartz & $600^{\circ} / 30 \mathrm{~min}$ \\
\hline \multirow[t]{3}{*}{ Aldrich $10 \%$ ZnS } & A & $10 \mu \mathrm{m}$ & Quartz & \\
\hline & $B$ & $10 \mu \mathrm{m}$ & Quartz & $400^{\circ} \mathrm{C} / 30 \mathrm{~min}$ \\
\hline & C & $10 \mu \mathrm{m}$ & Quartz & $600^{\circ} / 30 \mathrm{~min}$ \\
\hline Label & Sample ID & Particle Size & Substrate & Annealing Investigation \\
\hline \multirow[t]{5}{*}{ 120926A01 } & A & $9.5 \mathrm{~nm}$ & Alumina & Annealing \\
\hline & $B$ & $9.5 \mathrm{~nm}$ & Alumina & \multirow{4}{*}{$\begin{array}{l}\text { Films will be anneaed at } \\
400 \text { and } 600^{\circ} \mathrm{C} \text { in air and } \\
\text { Ar atmospheres }\end{array}$} \\
\hline & C & $9.5 \mathrm{~nm}$ & Alumina & \\
\hline & $D$ & $9.5 \mathrm{~nm}$ & Alumina & \\
\hline & $\mathrm{E}$ & $9.5 \mathrm{~nm}$ & Alumina & \\
\hline
\end{tabular}

Figure 1.62 shows the PL response of discrete $\mathrm{ZnS}$ particles $(9.5 \mathrm{~nm})$ annealed at different temperatures. A comparison with the PL response of as-processed particles and single crystal $\mathrm{ZnO}$ reference has also been made to evaluate the performance. The samples were excited with a $325 \mathrm{~nm}$ (He-Cd laser) optical signal for PL measurements, and the PL emission was detected with a photomultiplier tube. The photomultiplier tube (PMT) voltage was $401 \mathrm{~V}$, and not changed during the measurements. Data was taken from 330 to $850 \mathrm{~nm}$ in steps of $2 \mathrm{~nm}$, but cut off at $750 \mathrm{~nm}$ due to the insensitivity of the PMT above $750 \mathrm{~nm}$. The main points to note from Figure 1.62 are as follows:

- The $\mathrm{ZnO}$ reference sample shows near-band gap excitonic PL, a second, larger peak from a related defect, and a much stronger interband defect PL near $500 \mathrm{~nm}$.

- The ZnS samples showed different PL, depending on heat treatment. As synthesized showed some UV PL, starting at the $\mathrm{ZnO}$ band edge, decaying slowly into the band gap (lower energy/higher wavelength).

- Annealing at 200 and $400{ }^{\circ} \mathrm{C}$ changed the PL spectra, mainly by moving the peak energy to lower energy/higher wavelength. However, the $600^{\circ} \mathrm{C}$ anneal dramatically increased the PL; note that the PL signal (red) is 200 times that of the other ZnS samples, and 10 times that of the $\mathrm{ZnO}$. When the 325 laser was incident upon the $600^{\circ} \mathrm{C}$ annealed sample, the glow was really bright and appeared white.

- Detailed measurements were made to understand the origin of strong PL emission from the samples annealed at $600^{\circ} \mathrm{C}$ to further control the emission characteristics for optoelectronic applications. 
NF Nanoparticles $(9.5 \mathrm{~nm})$

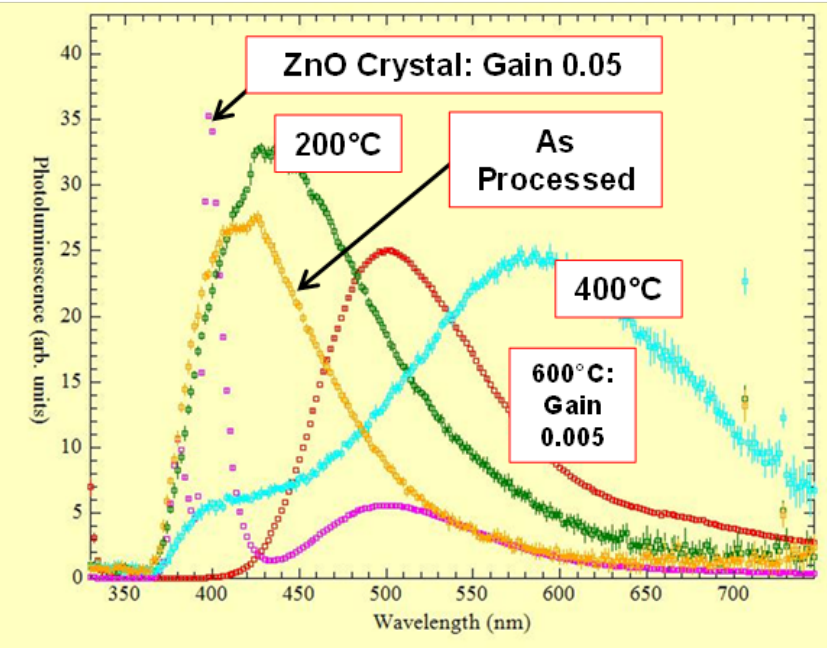

Figure 1.62. The PL response of as-deposited and annealed NF ZnS particles.

XRD measurements were also made to establish a correlation between the PL response and the phaseevolution. The NF ZnS sample was annealed in a box furnace in flowing $\mathrm{N}_{2}$ atmosphere. As shown in Figure 1.63, the $\mathrm{ZnS}$ sample changed to $\mathrm{ZnO}$ after annealing at $600^{\circ} \mathrm{C}$ possibly due to residual oxygen in the atmosphere. The nitrogen partial pressure was not sufficient to suppress oxidation. Based on the XRD results; further annealing studies were carried out in a tube furnace with controlled atmosphere. The impact of the annealing in inert gas and oxygen atmospheres on the NF ZnS properties was analyzed in detail as discussed in sections below, to understand and control the optical characteristics of NF ZnS nanoparticles.

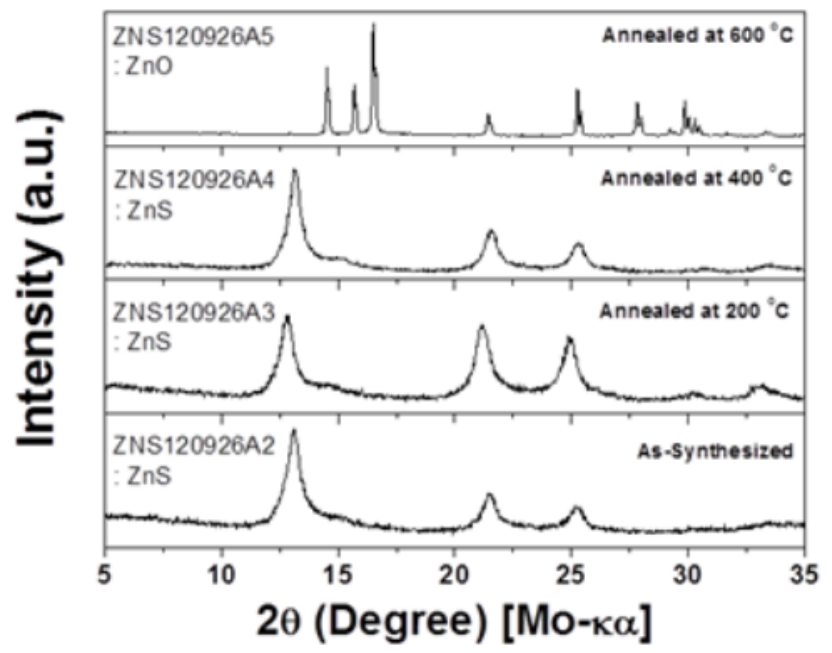

Figure 1.63. The XRD patterns of as-deposited and annealed NF ZnS particles (9.5nm).

\subsubsection{NF ZnS Thin Films}

An annealing study was conducted on NF ZnS (4.7nm) thin films to analyze the impact of temperature on the PL response and crystallinity. The films were annealed in a box furnace in flowing $\mathrm{N}_{2}$ atmosphere. The performance of the thin film samples was very similar to the annealed 
discrete particles. The XRD measurements, as shown in Figure 1.64, did not show the formation of $\mathrm{ZnO}$ phase up at $400^{\circ} \mathrm{C}$. However, annealing at $600^{\circ} \mathrm{C}$ resulted in a well-defined $\mathrm{ZnO}$ phase indicating that residual oxygen was enough to effectively oxidize the $\mathrm{ZnS}$ material. The as-deposited $\mathrm{ZnS}$ thin films showed a PL signal peaking at around 440nm. After annealing, the PL peak shifted to longer wavelengths. The XRD and PL results indicate that a control of annealing atmosphere is critical for $\mathrm{ZnS}$ material. At the same time the possibility of converting $\mathrm{ZnS}$ nanoparticle into $\mathrm{ZnO}$ nanoparticle opens up the possibility of targeting novel electronic applications exploiting its unique electronic properties.
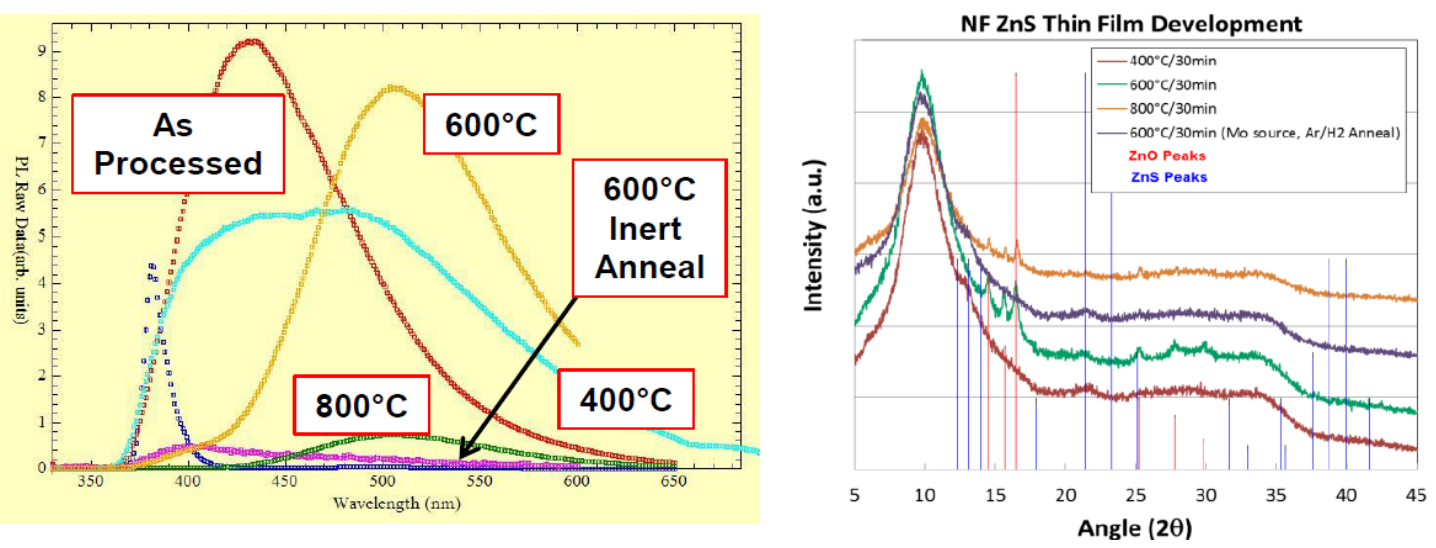

Figure 1.64. The impact of annealing on the (a) PL response and (b) crystallinity of NF ZnS thin films.

A number of $\mathrm{ZnS}$ thin film specimens were annealed in an inert atmosphere. The effects of the particle size on the microstructure and PL response were analyzed on the nanofermentation processed $(<10 \mathrm{~nm})$ and commercial $\mathrm{ZnS}$ particles $(\sim 10 \mu \mathrm{m})$. Figure 1.65 shows the PL response of the asprocessed NF ZnS thin films (9.5nm). The NF ZnS thin films showed a broad PL signal peaking at around $410 \mathrm{~nm}$ which matches well with the PL emission $(400-450 \mathrm{~nm})$ wavelengths typically reported for $\mathrm{ZnS}$ particles processed by chemical methods. The large commercial particles did not show any appreciable PL response that could be used as a reference for the present study.

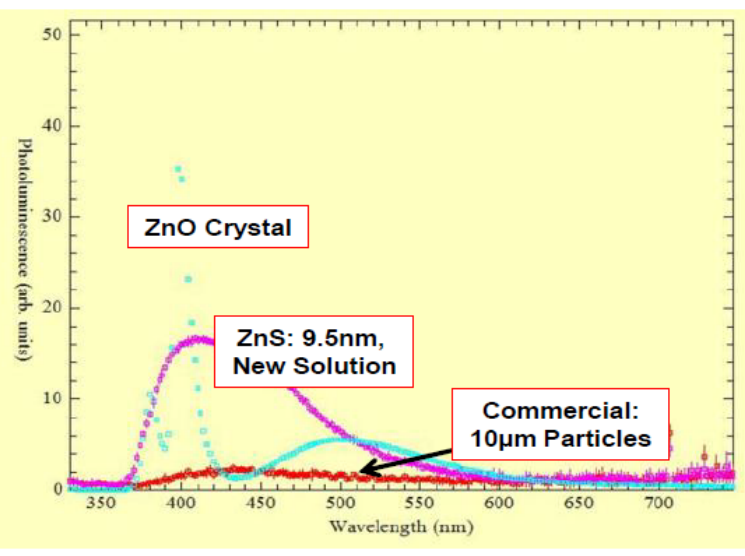

Figure 1.65. The typical PL response of asdeposited NF ZnS nanoparticles.

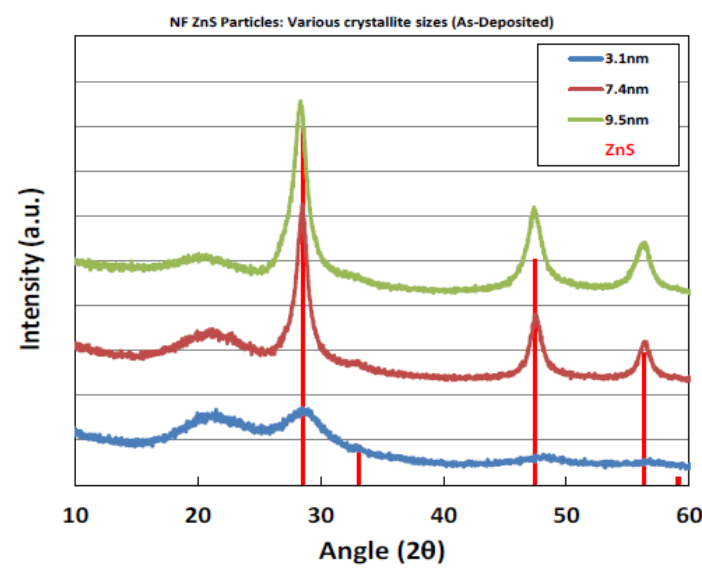

Figure 1.66. The XRD patterns of the asdeposited NF ZnS nanoparticle (3.1-9.5nm) thin films.

The NF ZnS thin films were annealed at various temperatures in a controlled inert and oxygen atmospheres to analyze the impact of annealing and particle size on the crystallinity and photoresponse characteristics. Figure 1.66 shows the XRD patterns of the as-processed NF ZnS 
nanoparticles (3.1-9.5 nm). The XRD patterns indicated the formation of a well-crystallized ZnS phase with no appreciable dependence on the particle size. The signal intensity was dictated by the material quantity exposed to the XRD beam rather than the material characteristics.

The NF ZnS thin films were also annealed in a pure oxygen atmosphere to analyze the impact of oxygen on the physical characteristics and crystallinity. As shown in Figure 1.67, the film color did not change appreciably after annealing in oxygen atmosphere. It may possibly be due to oxidation of the carbon and other impurities during annealing.

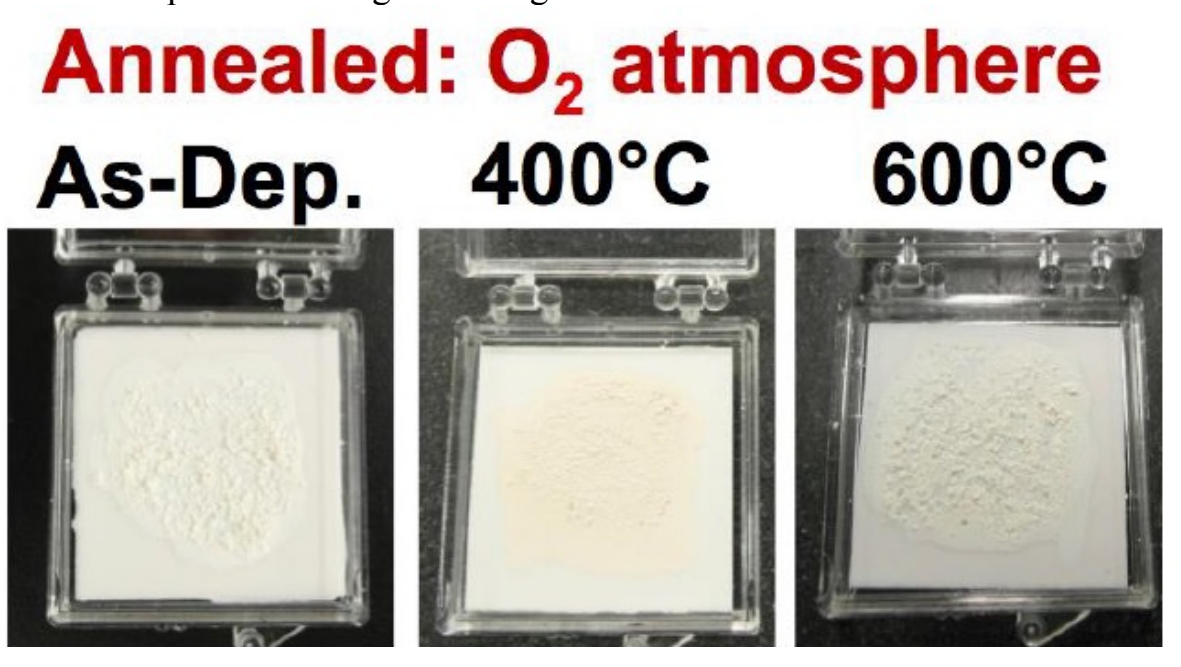

Figure 1.67. The NF ZnS thin films did not show any color change after annealing in an oxygen atmosphere as was observed after annealing in Ar atmosphere.

Figure 1.68 shows the XRD patterns of the films annealed in a pure oxygen atmosphere. The oxidation behavior of the NF ZnS thin film annealed in a $100 \%$ oxygen atmosphere appears to be similar to the films annealed in a box-furnace with residual oxygen. The $\mathrm{ZnS}$ phase is not significantly influenced by annealing at $400^{\circ} \mathrm{C}$. However, a highly crystalline $\mathrm{ZnO}$ phase is formed after annealing at $600^{\circ} \mathrm{C}$.

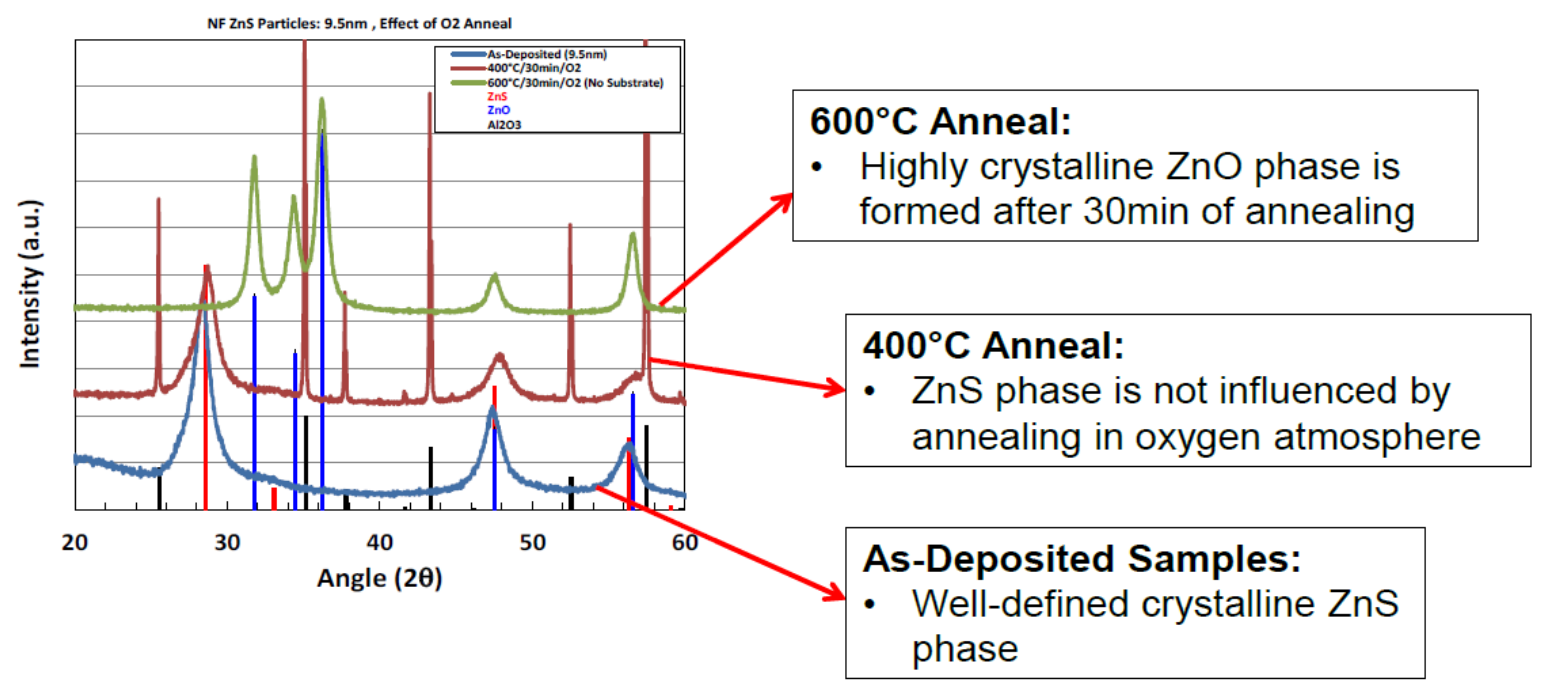

Figure 1.68. The impact of annealing in a pure oxygen atmosphere on the crystallinity and oxidation of NF ZnS thin films. 
The absolute QE of the sample is determined by comparing similar measurements of the laser beam with no sample in place with the spectral relative QE with the sample in place. Figure 1.69 shows the spectrum near $325 \mathrm{~nm}$ for the system with no sample (blue) and with the sample. The number of 325 $\mathrm{nm}$ photons is then proportional to the integration of the above equation for QE over the line width. We also show the spectrum of the laser line with the sample in place. The reflectivity of the sample can then be determined as the ratio of the integrals of the two lines, and is $7.2 \%$ for this sample.

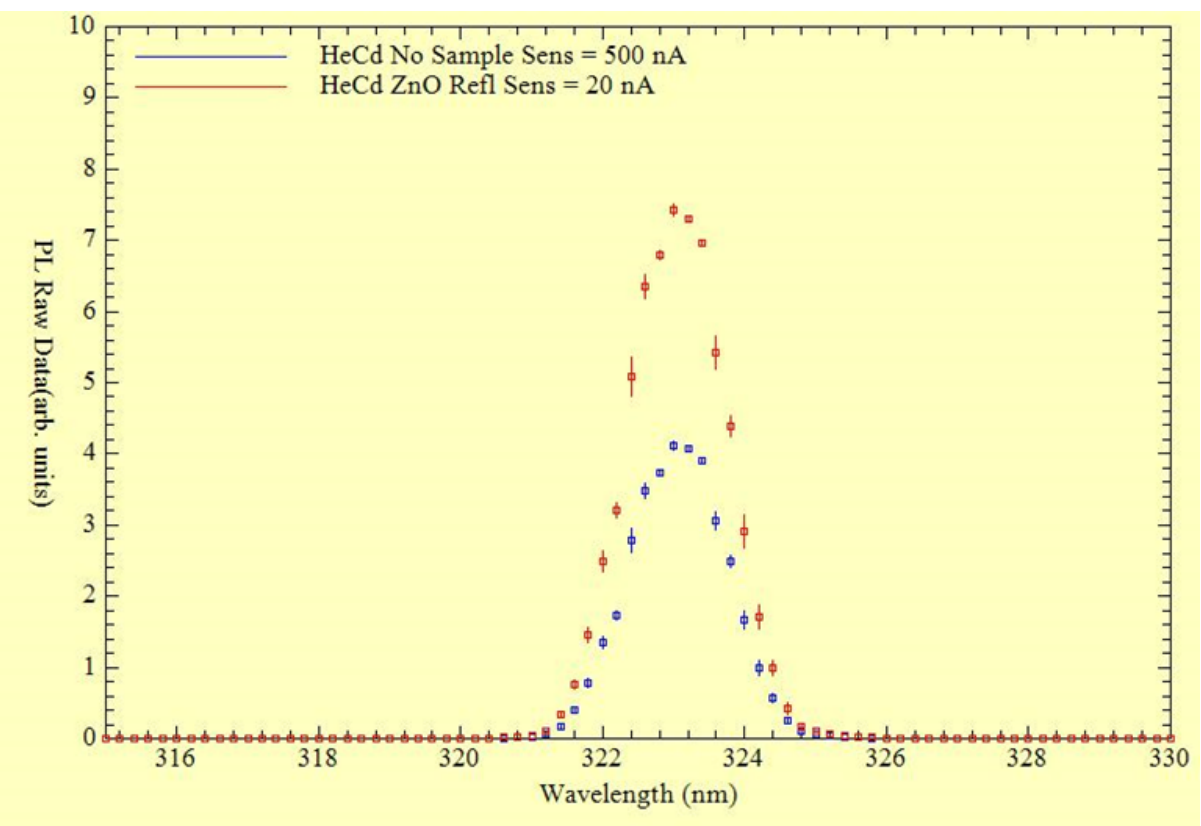

Figure 1.69. Photoluminescence spectrum with no sample (blue) and with a sample (red) in place.
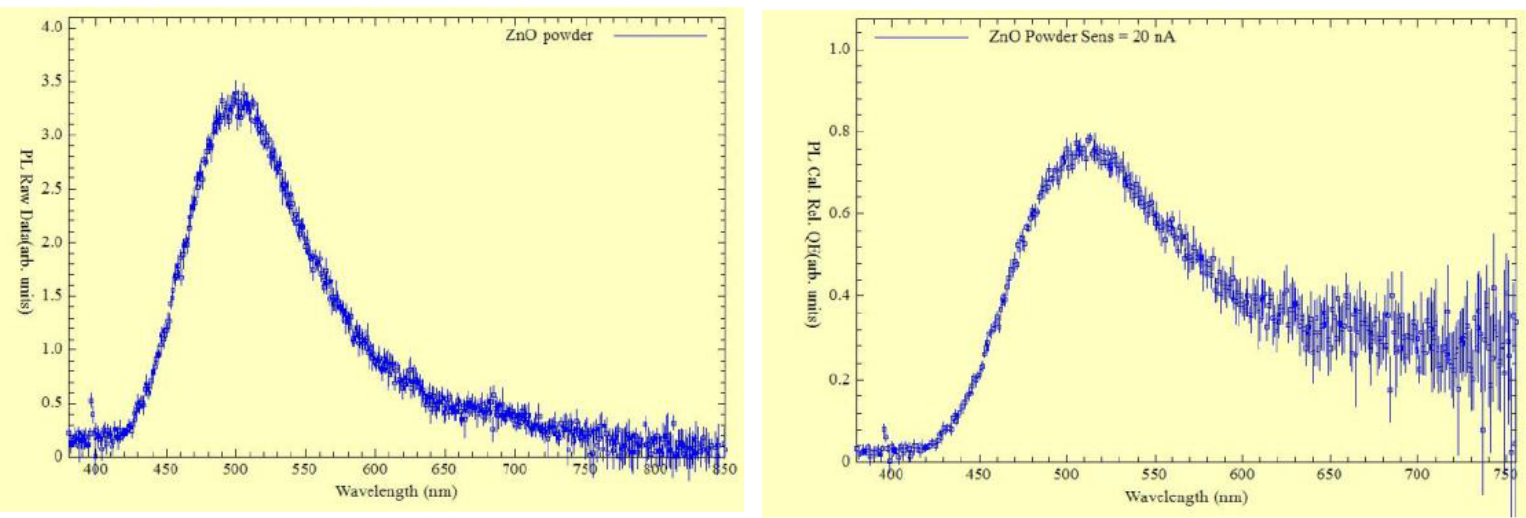

Figure 1.70. (a) - Raw photoluminescence data for ZnO sample, and (b) - Quantum efficiency data for ZnO sample.

Figure 1.70a shows the spectrum from the $\mathrm{ZnO}$ sample in raw data units and Figure 1.70b shows the same spectrum in relative QE units. The large errors in the data at long wavelengths are due primarily to the lower quantum efficiency of the PMT in this range, as well as the Wavelength multiplication factor in the equation above. By integrating the relative QE spectrum above from 400 to $750 \mathrm{~nm}$ and comparing with the integration over the $\mathrm{HeCd}$ spectral line (Fig. 1.69), we determine that the QE of the sample is $2.5 \%$. 


\subsubsection{Preparation of the ZnO NFNP target for low temperature senor deposition}

The deposition of NF $\mathrm{ZnO}$ was scalable with the pulse laser vaporization process, where the powder target was held in rotating holder in the vacuum chamber and laser pulses were used to remove material from the target and deposit it on preheated substrate. The deposition conditions were with a laser wavelength of $248 \mathrm{~nm}$ at $5 \mathrm{~Hz}$ with a total of 5000 pulses, and the target positioned at a $5 \mathrm{~cm}$ distance from the substrate. The nanofermented NP $\mathrm{ZnS}$ was converted to $\mathrm{ZnO}$ under heating at $1100 \mathrm{C}$ in air for 1 hour resulting in the formation of stoichiometric oxide. The target was prepared from prewashed $\mathrm{ZnO}$ compressed using 6 ton press (see Figure 1.71). An optical image of the target is shown in inset photograph of Figure 1.71.

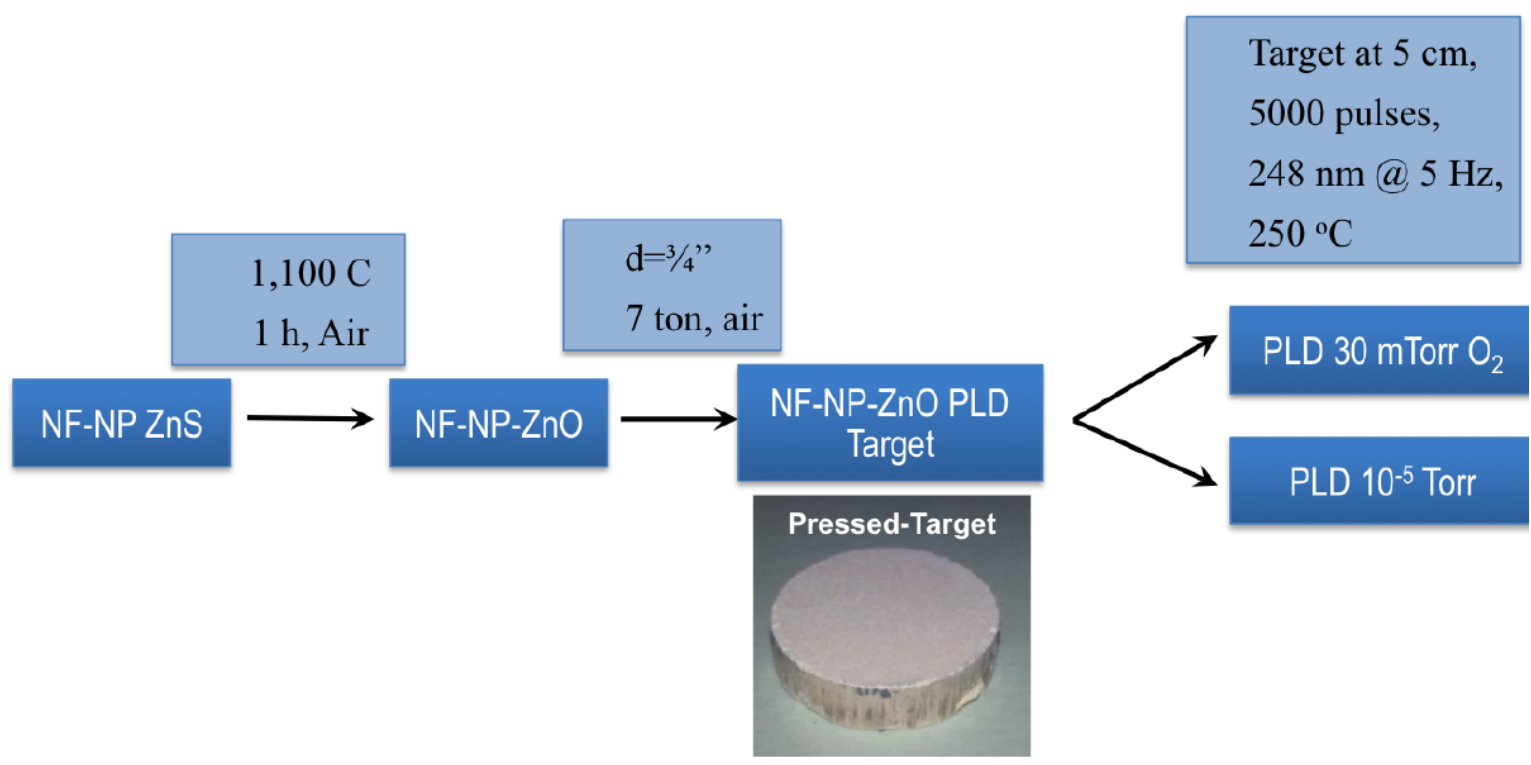

Figure 1.71. Flow chart of the sample preparation using a pressed target made from nanofermented NP.

The thin film of $\mathrm{ZnO}$ was deposited on the surface of pre-cleaned Si wafer with 150nm layer of thermal oxide to realize configuration of transistor with back gating. The films were deposited at two conditions (vacuum and 30mTorr of background oxygen. In both cases the substrate was preheated to 250 or $400 \mathrm{C}$ to assist with formation of continuous film of $\mathrm{ZnO}$. Figure 1.71 shows a flow chart of the sample preparation. Processing of NF-ZnO NP films is compatible with thin-film technologies (sputtering, PLD). This process is also compatible with low-cost, low-temperature processing thereby enabling flexible-electronics.

\subsection{THIN FILM ANNEALING AND CHARACTERIZATION - ZN GALLATE COATINGS}

The $\mathrm{Zn}$ Gallate coatings fabricated in the subtasks outlined above were annealed at various temperatures in normal air atmosphere. Figure 1.72 shows the $\mathrm{x}$-ray diffraction (XRD) patterns of the various films annealed at $950^{\circ} \mathrm{C}$. Both the $\mathrm{Zn}$ Gallate and substrate peaks are clearly visible in the XRD patterns. The Zn Gallate phase appears to be well-defined after annealing at $950^{\circ} \mathrm{C}$. The doping did not appear to have any appreciable impact on the XRD patterns. The lattice constant value was found to be in the range of $8.340-8.341 \AA$ for all the samples. 


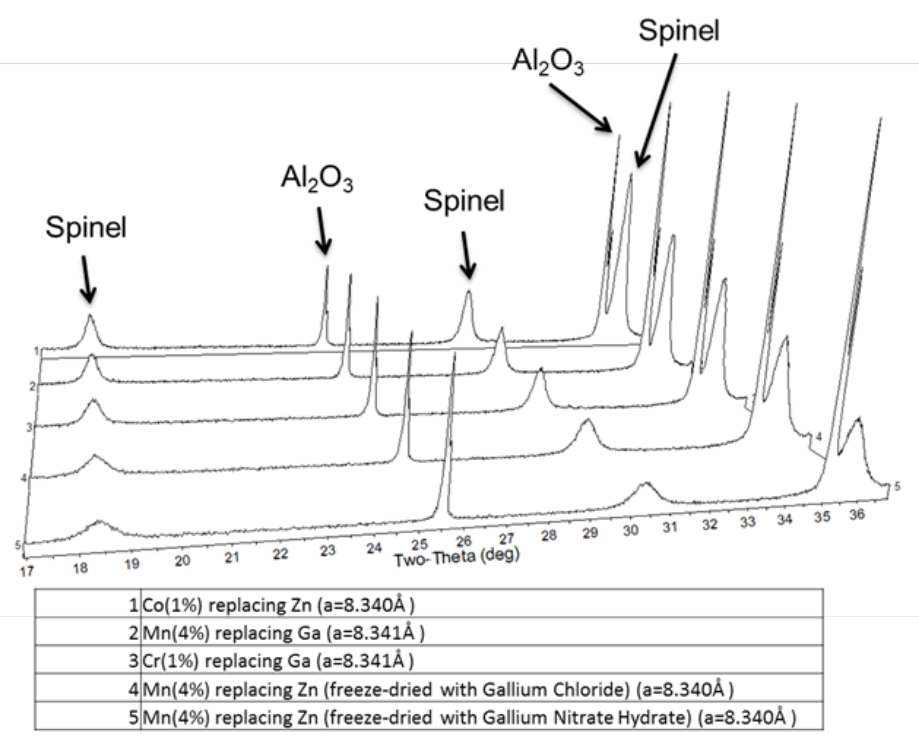

Figure 1.72. The X-ray diffraction patterns of the $\mathrm{ZnGa2O4} / \mathrm{Al} 2 \mathrm{O3}$ coatings annealed at $950^{\circ} \mathrm{C}$.
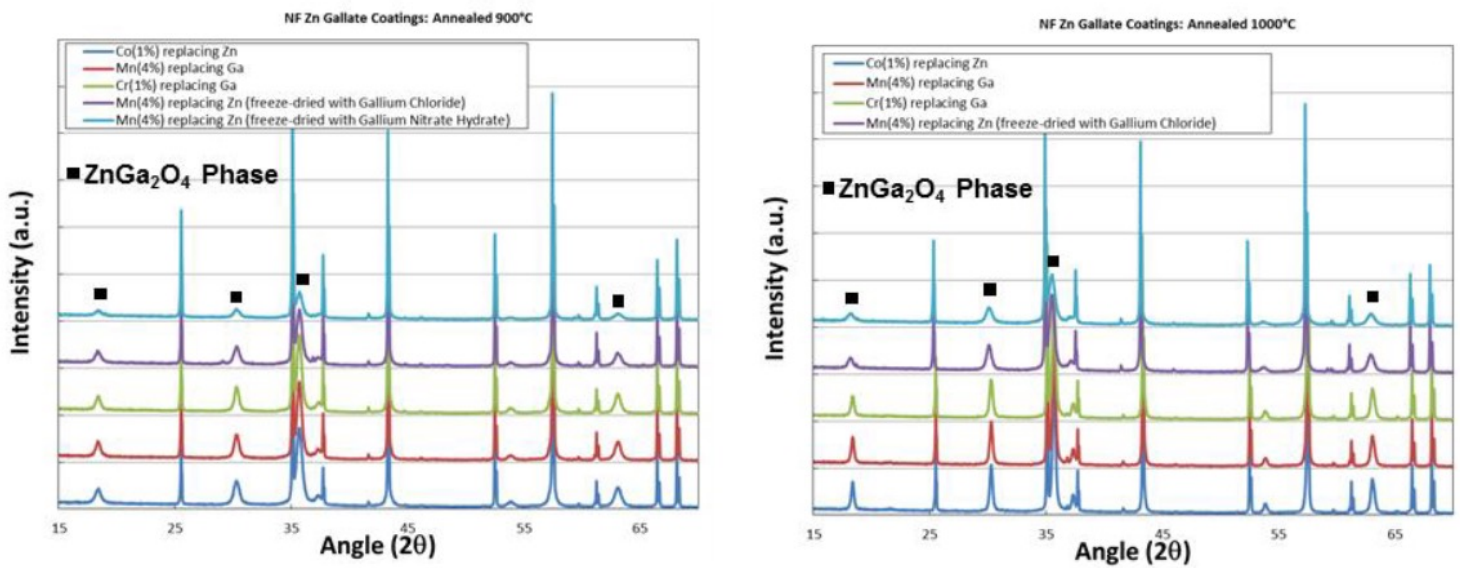

Figure 1.73. The $\mathrm{X}$-ray diffraction patterns of the $\mathrm{ZnGa} \mathrm{O}_{4} / \mathrm{Al}_{2} \mathrm{O}_{3}$ coatings annealed at various temperatures: (a) $900^{\circ} \mathrm{C}$, (b) $950^{\circ} \mathrm{C}$, and (c) $1000^{\circ} \mathrm{C}$. The unlabeled peaks are due to the substrate.

The XRD patterns of the coatings annealed at 900 and $1000^{\circ} \mathrm{C}$ are shown in Figure 1.73. The XRD patterns indicated the formation of a well-defined spinel phase at $900^{\circ} \mathrm{C}$. Annealing at a higher temperature of $1000^{\circ} \mathrm{C}$ resulted in sharper peaks for all the samples indicating an increase in the crystallinity of the Zn Gallate coatings. Further annealing study will be conducted in the expanded temperature range of $600-1100^{\circ} \mathrm{C}$ to gain a clear idea of the factors influencing the $\mathrm{Zn}$ Gallate phase formation, and the impact of crystallinity on the optical and electrical characteristics of the thick $\mathrm{Zn}$ Gallate coatings.

\subsubsection{Photoluminescence}


The photoluminescence characteristics of the $\mathrm{Zn}$ Gallate coatings annealed at $900^{\circ} \mathrm{C}$ are shown in Fig. 1.75. The PL measurements were conducted at room temperature. The PL was excited by a $325 \mathrm{~nm} \mathrm{He}-\mathrm{Cd}$ laser. The $\mathrm{ZnO}$ single crystal reference signal is also shown in Figure 1.74 for comparison. All the Zn Gallate coatings exhibited multiple peaks in the UV and visible part of the spectrum. The strongest PL response was observed for the $\mathrm{Cr}$ doped sample while the Mn doped samples showed the best response in the blue-green part of the spectrum, which is attractive for commercial applications.
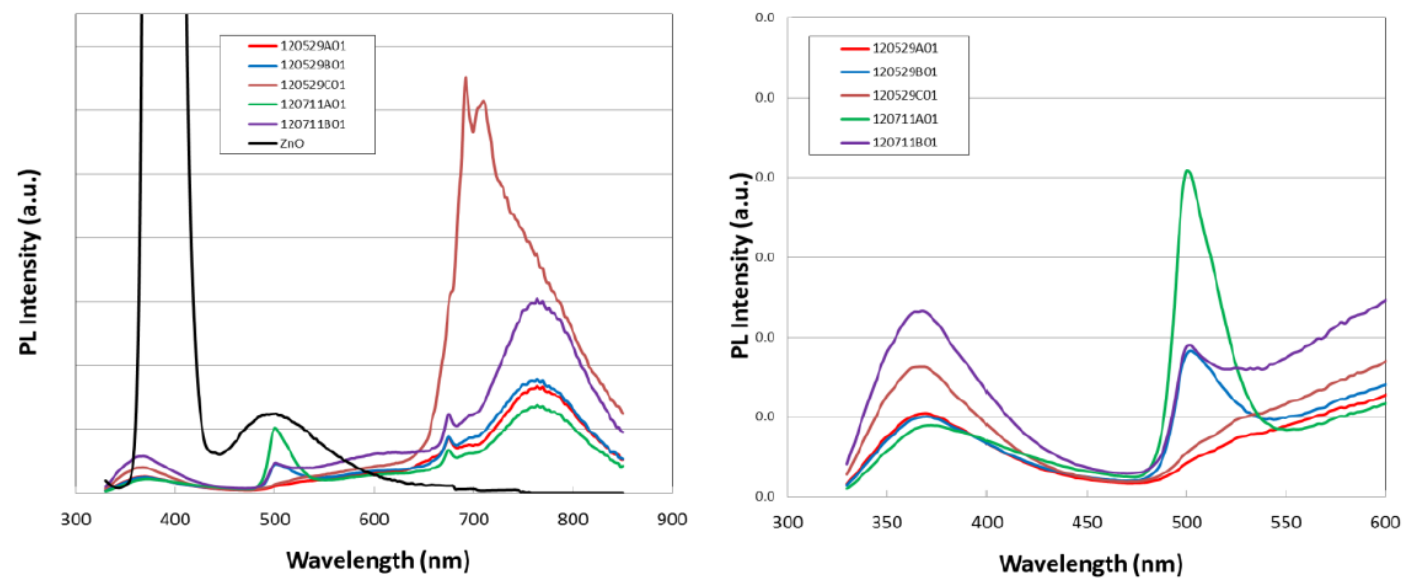

Figure 1.74. The PL response of various $\mathrm{ZnGa}_{2} \mathrm{O}_{4}$ coatings annealed at $900^{\circ} \mathrm{C}$ : (a) full spectrum (330850nm), (b) expanded view below $600 \mathrm{~nm}$. Sample details: $\bullet$ Co(1\%) replacing Zn, $\bullet$ Mn (4\%) replacing $\mathrm{Ga}, \bullet \mathrm{Cr}(1 \%)$ replacing $\mathrm{Ga}, \bullet \mathrm{Mn}(4 \%)$ replacing $\mathrm{Zn}$ (freeze dried with Gallium Chloride, $\bullet \mathrm{Mn}(4 \%)$ replacing Zn (freeze dried with Gallium Nitrate Hydrate)

As shown in Fig. 1.75, the PL intensity was found to increase with an increase in the annealing temperature in the range of $900-1000^{\circ} \mathrm{C}$. The observed results are consistent with the XRD patterns showing sharper peaks at higher annealing temperatures. The impacts of the annealing temperature and atmosphere will be analyzed in details to gain a clear understanding of the factors influencing the optical emission characteristics.

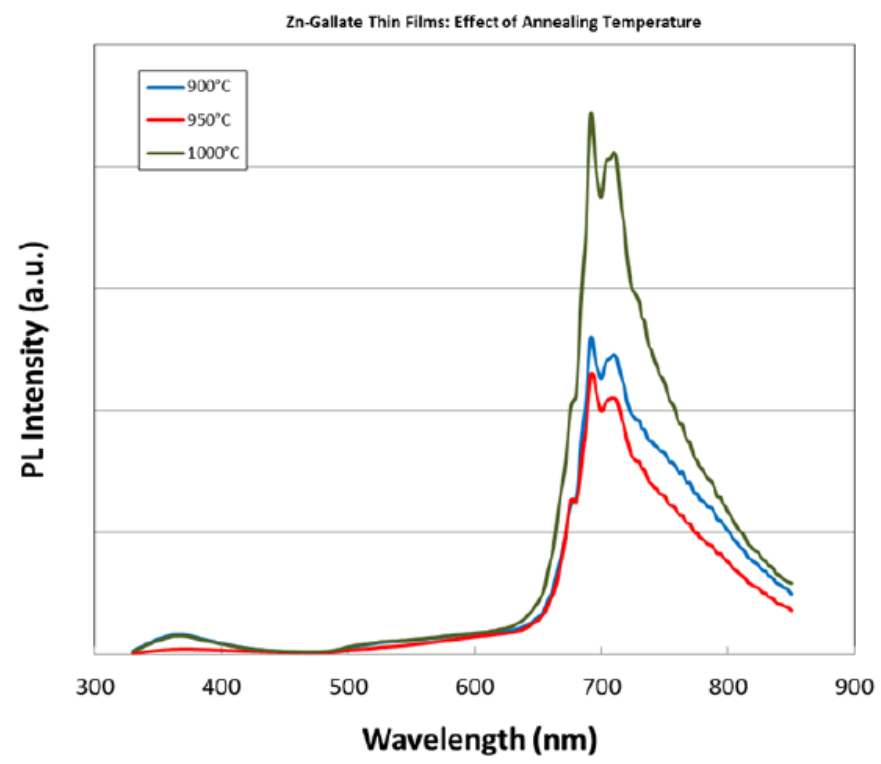

Figure 1.75. The effect of annealing temperature on the PL response of doped $\mathrm{ZnGa2O4}(1 \% \mathrm{Cr}$ 
replacing Ga) coatings.

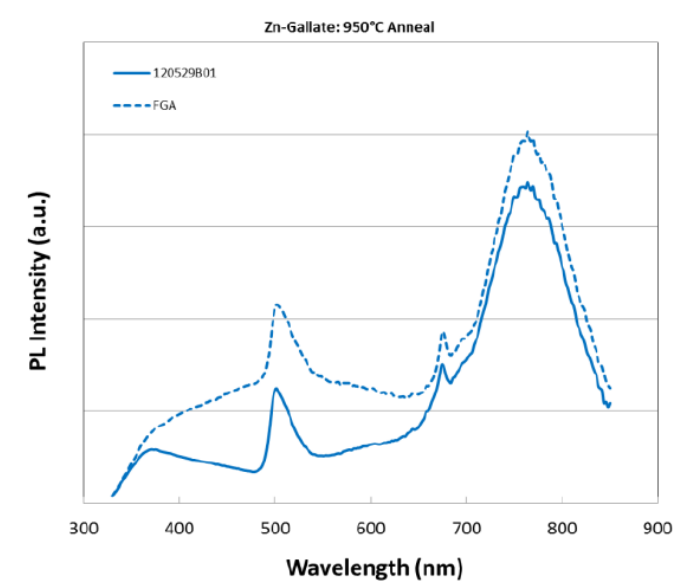

(a)

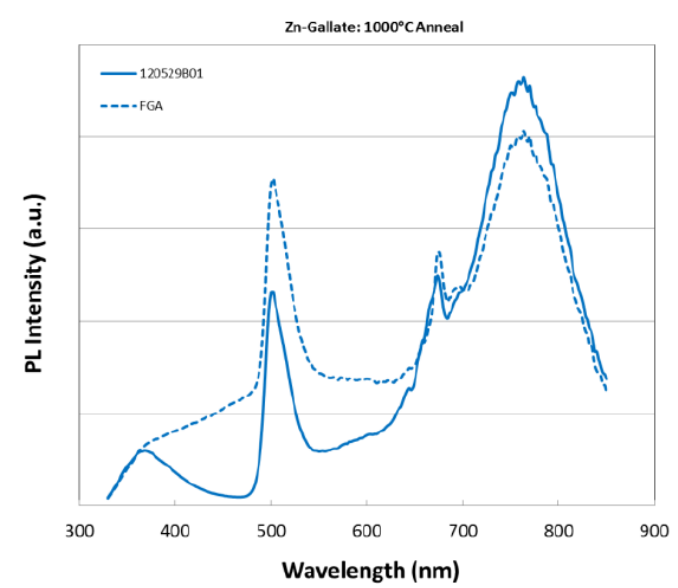

(b)

Figure 1.76. The effect of forming gas anneal on the $\mathrm{PL}$ response of doped $\mathrm{ZnGa}_{2} \mathrm{O}_{4}(\mathrm{Mn}(4 \%)$ replacing Ga) coatings annealed at (a) $950^{\circ} \mathrm{C}$ and (b) $1000^{\circ} \mathrm{C}$.

The effects of forming gas annealing (FGA) on the PL response of the Mn (4\%) doped Zn Gallate coatings were also analyzed as shown in Figure 1.76. The FGA was conducted at $450^{\circ} \mathrm{C}$ for 60 minutes in a flowing $\mathrm{Ar} / \mathrm{H}_{2}(4 \%)$ atmosphere. The FGA at a significantly lower temperature than the annealing temperature was effective in improving the PL response of the $\mathrm{Zn}$ Gallate coatings indicating that material defects are influencing the optical emission characteristics. A systematic FGA study was conducted to find the optimum processing window for defect passivation without deteriorating the material quality in a reducing hydrogen atmosphere.

The impact of doping on the PL emission characteristics of Zn Gallate films were investigated in detail as shown in Fig. 1.77, to develop a wide-band phosphor material. Cr doped $\mathrm{Zn}$ Gallate films were selected for the present study as they exhibited the strongest PL response among various doped films. The $\mathrm{Zn}$ Gallate material performance was being evaluated in terms of the ink stability, coating quality control, particle crystallinity, PL response of intrinsic/doped coatings, and electrical conductivity. The sample splits for the annealing investigation are listed in Table 1.15. The $\mathrm{Zn}$ Gallate coatings were processed in order to analyze the impact of post-deposition annealing temperature on the material structure and photoluminescence characteristics. The results of our investigation are presented below.

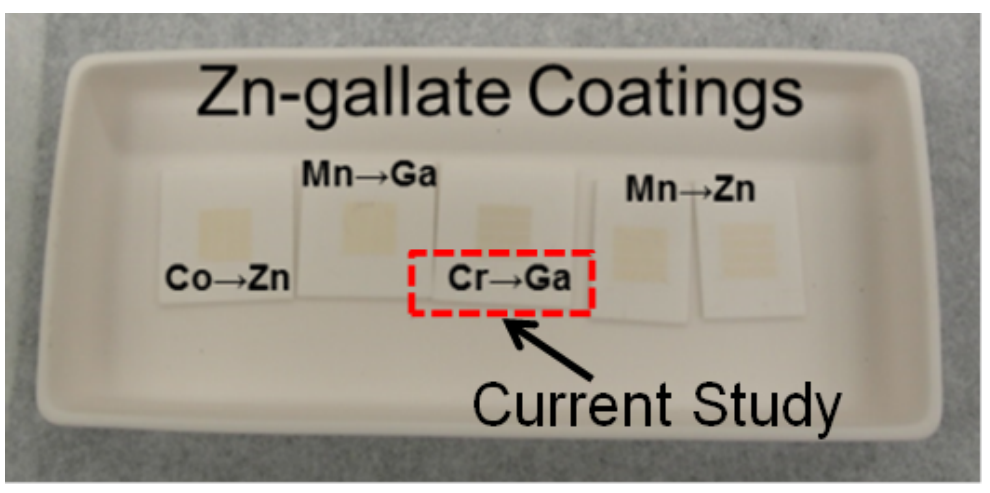


Figure 1.77. Various dopants under investigation for optical emission control for phosphor applications.

Table 1.15 Zinc Gallate sample splits for annealing investigation

\begin{tabular}{|l|l|l|l|}
\hline \multicolumn{1}{|c|}{$\begin{array}{c}\text { Sample ID } \\
\text { (Zn-Gallate, Cr replacing Ga (1\%) }\end{array}$} & Annealing & Atmosphere & $\begin{array}{c}\text { Forming Gas } \\
\text { Anneal }\end{array}$ \\
\hline ZCT100529C01_900CO & $900^{\circ} \mathrm{C} / 30 \mathrm{~min}$ & $\mathrm{O}_{2}$ & $450^{\circ} \mathrm{C} / 60 \mathrm{~min}$ \\
\hline ZCT100529C01_1000CO & $1000^{\circ} \mathrm{C} / 30 \mathrm{~min} \mathrm{O}_{2}$ & $450^{\circ} \mathrm{C} / 60 \mathrm{~min}$ \\
\hline ZCT100529C01_1100CO & $1100^{\circ} \mathrm{C} / 30 \mathrm{~min}$ & $\mathrm{O}_{2}$ & $450^{\circ} \mathrm{C} / 60 \mathrm{~min}$ \\
\hline ZCT100529C01_1100CA & $1100^{\circ} \mathrm{C} / 30 \mathrm{~min}$ & Air & $450^{\circ} \mathrm{C} / 60 \mathrm{~min}$ \\
\hline
\end{tabular}

\subsubsection{Crystallinity}

The $\mathrm{Cr}$ doped $\mathrm{Zn}$ Gallate films were annealed in the temperature range of $900-1100^{\circ} \mathrm{C}$ in air and pure oxygen atmospheres to analyze the impact of annealing atmosphere on the material structure. As shown in Fig. 1.78(a); the $\mathrm{Zn}$ Gallate spinel phase was well-formed at an annealing temperature of $900^{\circ} \mathrm{C}$. Only the stoichiometric $\mathrm{Zn}$ Gallate and substrate peaks were observed in the XRD patterns. The film crystallinity was found to improve with an increase in annealing temperature to $1000^{\circ} \mathrm{C}$. Annealing at a higher temperature of $1100^{\circ} \mathrm{C}$ resulted in substantially sharper peaks in the XRD pattern indicating significant improvement in film crystallinity and grain growth. Similar XRD patterns were observed for the films annealed in air and oxygen atmospheres; as shown in Fig. 1.78(b), indicating that the as processed nanoparticle films have sufficient oxygen for a stoichiometric phase formation. The x-ray photoelectron spectroscopy (XPS) measurements will be conducted to further analyze the elemental composition of the films and correlate it to the observed XRD results for a clear understanding of the phase formation kinetics.

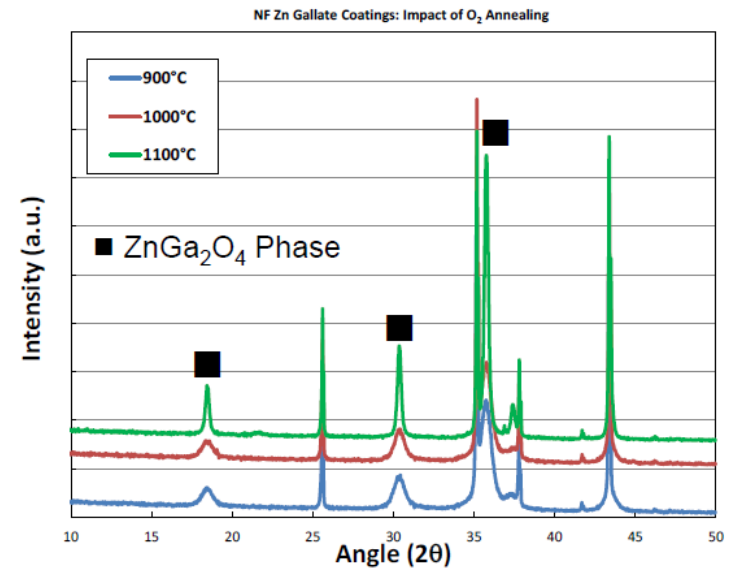

(a)

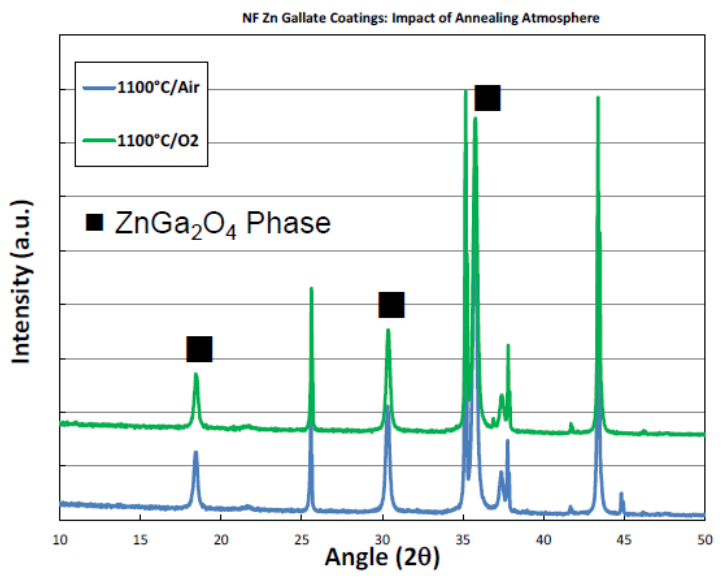

(b)

Figure 1.78. The $x$-ray diffraction patterns of the $\mathrm{ZnGa}_{2} \mathrm{O}_{4} / \mathrm{Al}_{2} \mathrm{O}_{3}$ coatings annealed at various temperatures: (a) $900-1100^{\circ}$ anneal in oxygen atmosphere, (b) $1100^{\circ} \mathrm{C}$ anneal in air and oxygen atmospheres.

\subsubsection{Photoluminescence of Annealed Coatings}

The photoluminescence characteristics of the annealed $\mathrm{Cr}$ doped $\mathrm{Zn}$ Gallate coatings are shown in Fig. 1.79. The films were annealed in the temperature range of $900-1100^{\circ} \mathrm{C}$ in a flowing oxygen 
atmosphere. The PL measurements were conducted at room temperature using a $325 \mathrm{~nm}$ He-Cd laser for excitation. The Cr doped Zn Gallate films exhibited strong PL response in the red part of the spectrum. The PL response was not influenced by the annealing atmosphere nor the annealing temperature in the range of $900-1100^{\circ} \mathrm{C}$, while the XRD patterns indicated a substantial improvement in film crystallinity. A correlation among the PL response, material crystallinity, and dopant concentration need to be established to further improve the photoemission characteristics; which will be the focus of our future investigation.

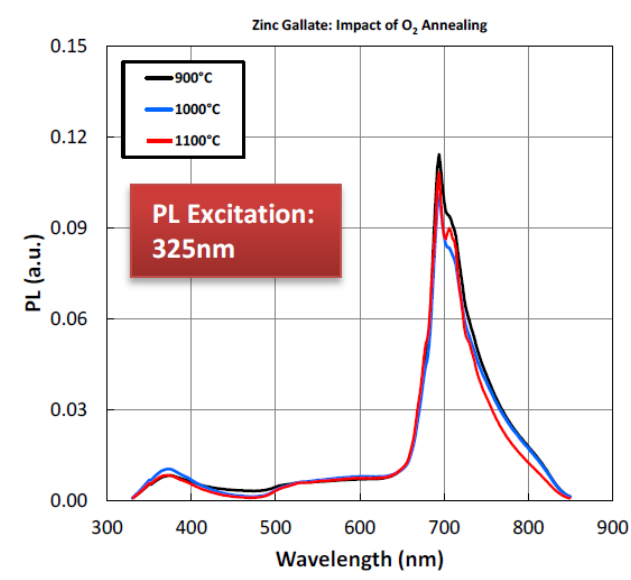

(a)

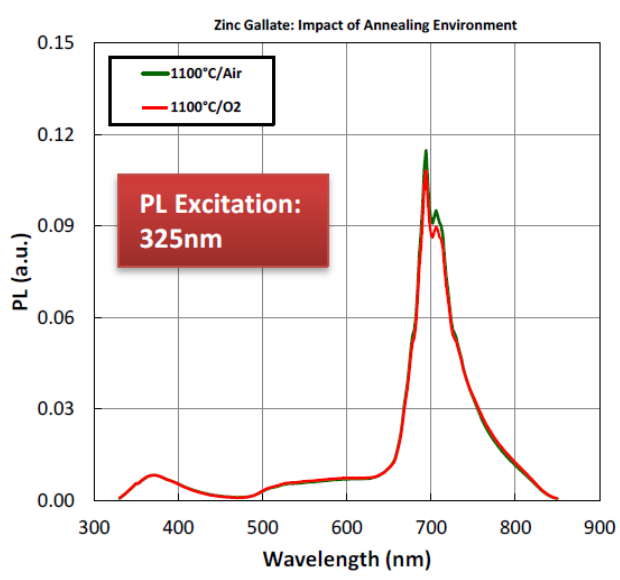

(b)

Figure 1.79. The PL response of the $\mathrm{ZnGa}_{2} \mathrm{O}_{4}$ coatings annealed at various temperatures: (a) $900-1100^{\circ}$ anneal in oxygen atmosphere, (b) $1100^{\circ} \mathrm{C}$ anneal in air and oxygen atmospheres.

The effects of forming gas annealing (FGA) on the PL response of the $\mathrm{Cr}$ doped $\mathrm{Zn}$ Gallate coatings were also analyzed as shown in Fig. 1.80. The FGA was conducted at $450^{\circ} \mathrm{C}$ for 60 minutes in a flowing $\mathrm{Ar} / \mathrm{H}_{2}(4 \%)$ atmosphere. The FGA did not result in any appreciable improvement of the PL response of the Cr doped $\mathrm{Zn}$ Gallate films. The PL response was found to be similar for both the air and oxygen atmosphere annealed films. The observed PL response suggests that the optical emission characteristics were not limited by defect related absorptions in the bulk of the material. We will continue our investigation of the defect structure and defect-passivation in various intrinsic and doped Zn Gallate films for the development of an efficient phosphor.

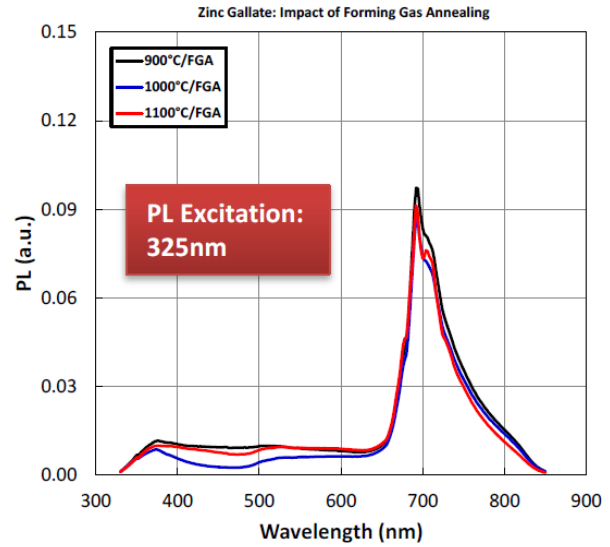

(a)

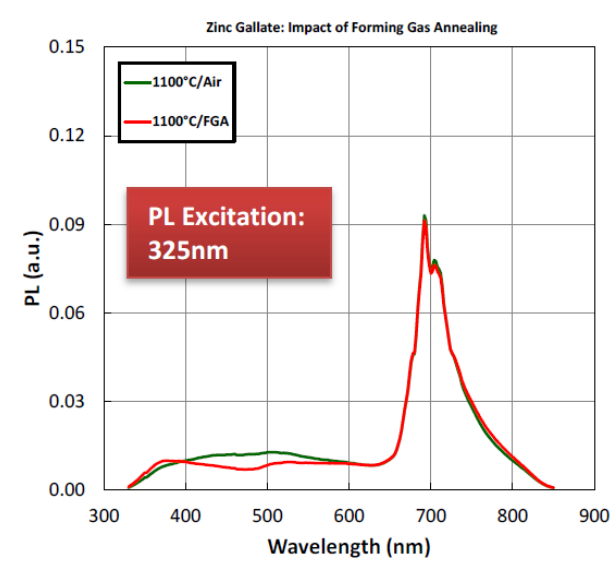

(b)

Figure 1.80. The impact of forming gas anneal on PL response of the $\mathrm{ZnGa}_{2} \mathrm{O}_{4}$ coatings processed at various temperatures: (a) $900-1100^{\circ}$ anneal in oxygen atmosphere, (b) $1100^{\circ} \mathrm{C}$ anneal in air and oxygen atmospheres. 


\subsubsection{PL Quantum Efficiency}

The PL quantum efficiency (PLQE) measurements were conducted both on Cr doped Zn Gallate films deposited on alumina and silica substrates and on Cr-doped Zn Gallate powders. The films were annealed at a temperature of $1000^{\circ} \mathrm{C}$. The $\mathrm{Cr}$ doped $\mathrm{Zn}$ Gallate films deposited on alumina substrates exhibited appreciable background signal resulting from the PL from the substrate. As a result, the evaluation of the material PLQE was determined using Cr-doped $\mathrm{Zn}$ Gallate samples formed on fused silica substrates. The resulting PLQE (600-850 nm) was $0.95 \pm 0.07 \%$ using PL excitation of $325 \mathrm{~nm}$. If Cr-doped Zn Gallate powder samples are examined, then a PLQE (600-850 $\mathrm{nm})$ of $1.9 \pm 0.2 \%$ was obtained.

The photoluminescence measurements continued with 4 different samples of zinc gallate powder as listed in Table 1.16. All the samples were annealed at a temperature of $1100^{\circ} \mathrm{C}$ for 30 minutes in a flowing oxygen atmosphere. The impact of nanofermentation process on the zinc gallate particles quality was analyzed in terms of the photo-response characteristics. The photo-response of the zinc gallate material was evaluated in terms of the photoluminescence (PL) characteristics. The PL

illumination wavelength was $325 \mathrm{~nm}$ and the optical configuration was without the integrating sphere.

Table 1.16 Zinc Gallate sample splits for PL investigation. All samples were annealed in $\mathrm{O}_{2}$ atmosphere

\begin{tabular}{|l|l|l|l|}
\hline \multicolumn{1}{|c|}{ Sample ID } & $\begin{array}{c}\text { Crystallite } \\
\text { Size }\end{array}$ & $\begin{array}{c}\text { Annealing } \\
\text { Temperature/Time }\end{array}$ & \multicolumn{1}{|c|}{ NF Process Details } \\
\hline ZGT130716 A01 & $3.2 \pm 0.1 \mathrm{~nm}$ & $1100^{\circ} \mathrm{C} / 30 \mathrm{~min}$ & $\begin{array}{l}\text { All (Precursor }+ \text { MOPS ) at } \\
\text { initial 2 weeks }\end{array}$ \\
\hline ZGT130716 B01 & $3.0 \pm 0.1 \mathrm{~nm}$ & $1100^{\circ} \mathrm{C} / 30 \mathrm{~min}$ & $\begin{array}{l}\text { (precursor }+ \text { MOPS) after } 1 \\
\text { day for 1 week }\end{array}$ \\
\hline ZGT130716 C01 & $3.0 \pm 0.1 \mathrm{~nm}$ & $1100^{\circ} \mathrm{C} / 30 \mathrm{~min}$ & $\begin{array}{l}\text { (precursor }+ \text { MOPS) after } 1 \\
\text { day for 2 week }\end{array}$ \\
\hline ZGT130716 D01 & $3.4 \pm 0.1 \mathrm{~nm}$ & $1100^{\circ} \mathrm{C} / 30 \mathrm{~min}$ & $\begin{array}{l}\text { (precursor }+ \text { NaOH) after } 1 \\
\text { day for 1 week }\end{array}$ \\
\hline
\end{tabular}

The plot of the photoluminescence is shown in Figure 1.81. As can be seen, all samples show a strong PL around $500 \mathrm{~nm}$, and another series of PL peaks in the $670 \mathrm{~nm}$ range. Note that the $670 \mathrm{~nm}$ $\mathrm{PL}$ is real and not due to second order diffraction of the $325 \mathrm{~nm}$ laser line, since a color filter is used to eliminate the second order light. Figure 5 documents the photoluminescence response for various annealing conditions of $\mathrm{Zn}$ gallate thin films. 


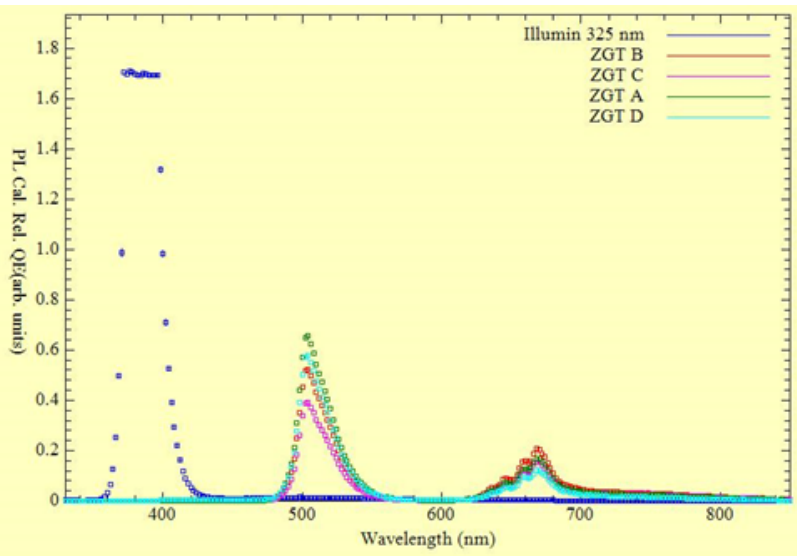

Figure 1.81. Photoluminescence from 4 different zinc gallate samples, and the photoluminescence from a standard ZnO sample (blue and off scale).

The PL system was then re-configured to obtain the quantum efficiency using the technique described in a previous report. Here, the $325 \mathrm{~nm}$ laser beam is used for illumination, but the sample powder is inserted into an integrating sphere so that all light emitted from the sample can be detected. As with all integrating sphere measurements, the signal was considerably less than the open-air experiments used for the data shown in Fig. 1.81. To improve the signal-to-noise, the slit on the monochromator was increased to $1 \mathrm{~mm}$. Figure 1.82 shows the light intensity collected without the sample and with the sample near the $325 \mathrm{~nm}$ laser line. By integrating the intensities, the reflectivity of the powder was determined to be $38.0 \pm 0.1 \%$.

Figure 1.83 shows the PL of zinc gallate in the integrating sphere. The signal strength of the data shown in Fig. 1.83 was considerably less than that of Fig 1.81 by a factor of $10^{4}$, resulting in the increased noise as shown in Fig. 1.83. By taking the ratio of the integrated intensity of the data in Fig. 1.83 compared to the intensity of the laser light (Fig. 1.82), we get the quantum efficiency (QE) of $1.02 \pm 0.01 \%$.

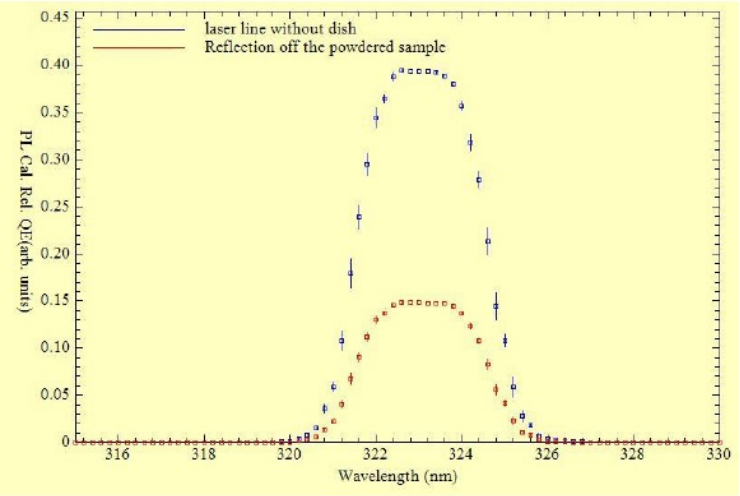

Figure 1.82. The impact of sample reflectivity on the collected PL excitation signal.

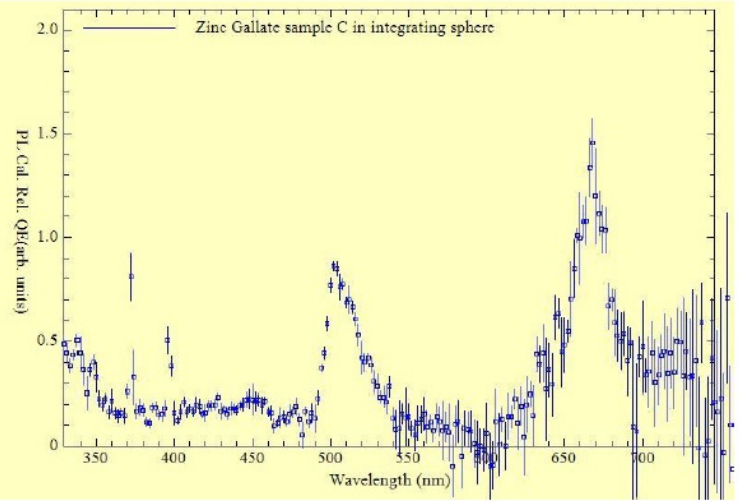

Figure 1.83. Photoluminescence of Zinc Gallate taken in the quantum efficiency configuration.

\subsubsection{Effect of Annealing on Zinc Gallate Crystallinity}

The samples listed in Table 1.17 were annealed at $1100^{\circ} \mathrm{C}$ for 30 minutes to enhance crystallinity and $\mathrm{PL}$ response characteristics. For the present batch of samples, the $\mathrm{Zn} / \mathrm{Mn} / \mathrm{Ga}$ ratio was varied to analyze the impact of divalent cation concentration on the microstructure and PL response. As shown 
in Figure 1.84, all the samples exhibited a well-defined crystallized $\mathrm{ZnGa}_{2} \mathrm{O}_{4}$ phase. The XRD peak intensity and sharpness were found to improve significantly as a result of annealing. The Ga-O and $\mathrm{GaO}(\mathrm{OH})$ phases were also observed for the present samples.

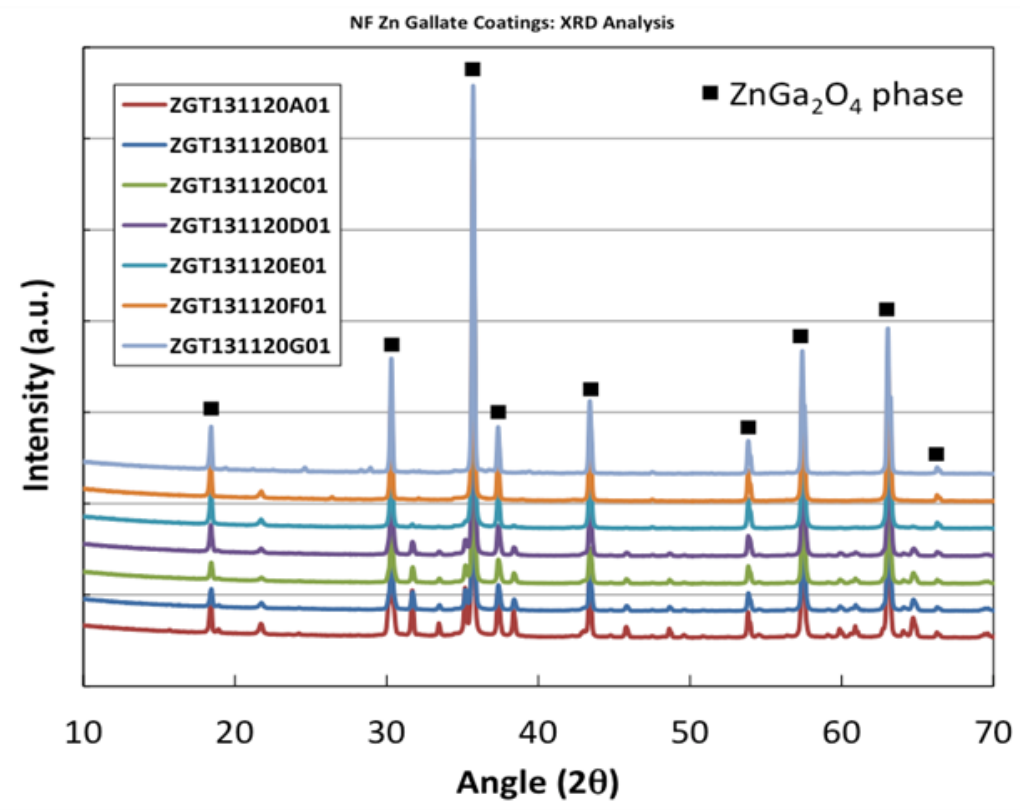

Figure 1.84. X-ray diffraction patterns of annealed zinc gallate particles with various $([\mathrm{Zn}]+[\mathrm{Mn}]) /[\mathrm{Ga}]$ ratios.

\subsubsection{Photoluminescence of Zinc Gallate Powder Made by Nanofermentation}

Photoluminescence (PL) studies were performed on several zinc gallate (ZGT) samples ZGT131120A-G, where the last letter indicates a variation in the composition of the samples. The primary compositional variation in samples $A-G$ is the zinc to gallium ratio $\left(\mathrm{Zn}_{1 \pm 0.3}: \mathrm{Ga}_{2}\right)$, where all samples are doped with $\sim 4 \% \mathrm{Mn}$ All samples had been annealed at $1100{ }^{\circ} \mathrm{C}$ for $30 \mathrm{~min}$. These are briefly described in Table 1.17 with a more complete description given below. For comparison purposes, the PL from a trichromatic phosphor (used in fluorescent lamps) was also examined. All samples were in the form of a powder.

Table 1.17 ZGT131120 samples examined using photoluminescence

\begin{tabular}{|l|l|l|}
\hline Sample & Stoichiometry & Ave. Size (nm) \\
\hline A & $\mathrm{Zn}_{0.7} \mathrm{Ga}_{2}$ & $2.5 \pm 0.1$ \\
\hline $\mathrm{B}$ & $\mathrm{Zn}_{0.8} \mathrm{Ga}_{2}$ & $2.8 \pm 0.1$ \\
\hline $\mathrm{C}$ & $\mathrm{Zn}_{0.9} \mathrm{Ga}_{2}$ & $3.0 \pm 0.2$ \\
\hline $\mathrm{D}$ & $\mathrm{Zn}_{1.0} \mathrm{Ga}_{2}$ & $3.0 \pm 0.1$ \\
\hline $\mathrm{E}$ & $\mathrm{Zn}_{1.1} \mathrm{Ga}_{2}$ & $3.6 \pm 0.2$ \\
\hline $\mathrm{F}$ & $\mathrm{Zn}_{1.2} \mathrm{Ga}_{2}$ & $3.5 \pm 0.1$ \\
\hline $\mathrm{G}$ & $\mathrm{Zn}_{1.3} \mathrm{Ga}_{2}$ & $3.8 \pm 0.2$ \\
\hline
\end{tabular}

The ZGT samples as presented contained very little material, so it was not possible to perform variable excitation samples using the Fluorimeter at CNMS. Therefore, we performed the PL measurements using the system in the CATS lab. Two excitation sources were used; a HeCd laser (325 nm) and a white light $\mathrm{HgXe}$ arc lamp. The $\mathrm{HgXe}$ arc lamp emits a broad spectrum of light with 
significant intensity in many of the standard $\mathrm{Hg}$ lines. We used standard narrow-pass interference filters (10 $\mathrm{nm}$ full width at half max band width, $\sim 15 \%$ maximum transmission) to select out the specific wavelengths used to illuminate the sample. The HeCd laser emits $\sim 20 \mathrm{~mW}$ of light energy, while the energy of the light from the filtered $\mathrm{HgXe}$ lamp was nearly 3 orders of magnitude less.

Four $\mathrm{Hg}$ lines were examined: 1) $254 \mathrm{~nm}(11 \mu \mathrm{W}), 2) 313 \mathrm{~nm}(56 \mu \mathrm{W}), 3) 365 \mathrm{~nm}(61 \mu \mathrm{W})$, and 4) $405 \mathrm{~nm}(18 \mu \mathrm{W})$.

The small amount of sample also precluded us from performing integrating sphere measurements to determine the quantum efficiency (QE) of the emitted PL. As a result, we compared the PL from the ZGT samples with the trichromatic phosphor, and report the QE relative to the trichromatic phosphor. No corrections were made for reflection losses.

Figure 1.85 shows the PL for the trichromatic sample using the 4 different illumination energies from the HgXe lamp, normalized for the number of illumination photons. The spectra were taken from $330-850 \mathrm{~nm}$, so the $254 \mathrm{~nm}$ and $313 \mathrm{~nm}$ data do not show the excitation source, but the $365 \mathrm{~nm}$ and $405 \mathrm{~nm}$ spectra do show a significant peak at the illumination wavelength (this is just the reflected light from the powder). The trichromatic phosphor was designed to operate using $254 \mathrm{~nm}$ excitation, and it contained $\mathrm{Y}_{2} \mathrm{O}_{3}:$ Eu, which emits at $611 \mathrm{~nm}$ and several other red wavelengths when illuminated with $254 \mathrm{~nm}$ light. These emission wavelengths were not present when the trichromatic was illuminated using longer wavelengths, since the $\mathrm{Y}_{2} \mathrm{O}_{3}$ :Eu component was not excited. The broad blue PL is strongly excited by both $313 \mathrm{~nm}$ and $365 \mathrm{~nm}$ light.

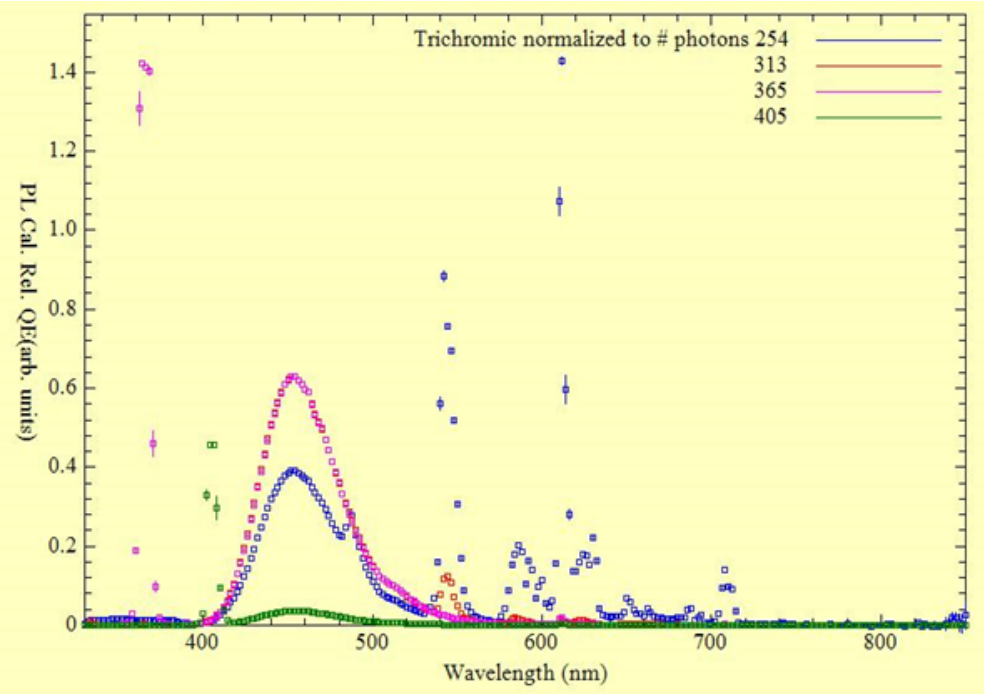

Figure 1.85. Photoluminescence spectra from the trichromatic phosphor using 4 different excitation wavelengths. 

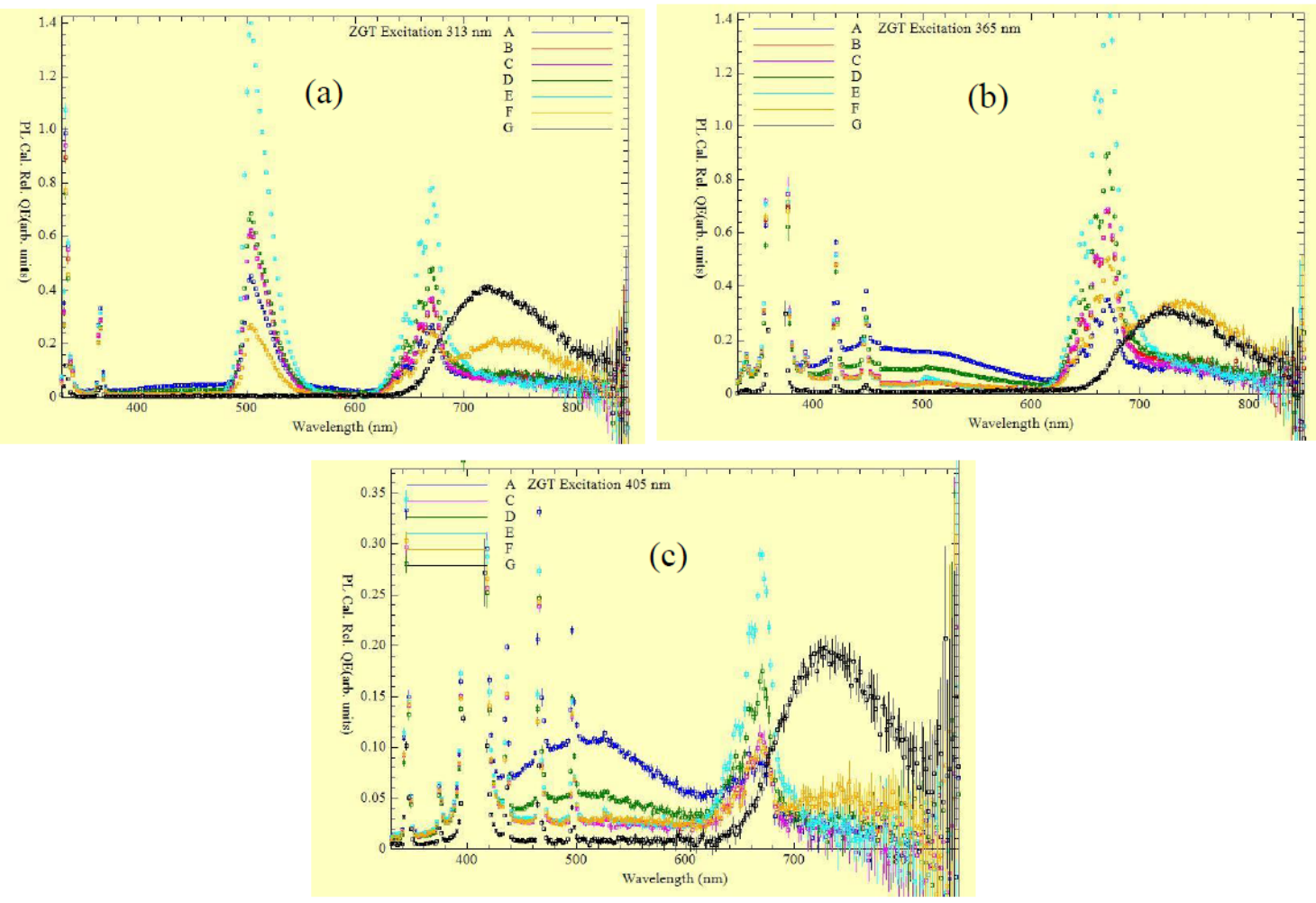

Figure 1.86. Photoluminescence from the various ZGT samples when illuminated using various wavelengths from the HgXe lamp. Illumination using the $254 \mathrm{~nm}$ wavelength showed no observable PL, so it is not shown.

No observable PL was observed from the ZGT samples when excited by $254 \mathrm{~nm}$ light, but significant PL was observed when excited using $313 \mathrm{~nm}, 365 \mathrm{~nm}$, and $405 \mathrm{~nm}$ light. This data is shown in Figure 1.86a. When illuminated using $313 \mathrm{~nm}$ excitation, all samples exhibited a strong peak near $338 \mathrm{~nm}$ (A-F; $334 \mathrm{~nm}$ for G) and $365 \mathrm{~nm}$ (A-F, weak for G). Strong broad transitions were observed near $505 \mathrm{~nm}$ and $670 \mathrm{~nm}$ arising from transitions in $\mathrm{Mn}^{+2}$ in samples A-F, while samples $\mathrm{G}$ and $\mathrm{F}$ exhibit a very broad transition at $730 \mathrm{~nm}$. Sample G did not show the broad transitions at $505 \mathrm{~nm}$ and $670 \mathrm{~nm}$.

If the samples were illuminated using $365 \mathrm{~nm}$ light (Figure 1.86b), the broad transition at $670 \mathrm{~nm}$ was still observable for samples A-F, but the $505 \mathrm{~nm}$ transition was absent. Sharp transitions were also observable at $420 \mathrm{~nm}$ and $448 \mathrm{~nm}$ (weak for sample G). Interestingly, samples A-F show a transition at higher energy (lower wavelength) at $338 \mathrm{~nm}$ for samples A-F, while this transition was weaker and at slightly lower energy for sample G. The broad transition at $730 \mathrm{~nm}$ was still observable for samples F and G. Samples A and D also showed a very broad PL centered at $450 \mathrm{~nm}$.

If the samples were illuminated with $405 \mathrm{~nm}$ light (see Figure 1.86c), the $670 \mathrm{~nm}$ transition was still observable for samples A-F, and the 730 transition is observable for sample G. Two higher energy transitions were observable at $344 \mathrm{~nm}$ and $374 \mathrm{~nm}$, while lower energy transitions were observable at 436, 466, and $496 \mathrm{~nm}$. A broad PL at $500 \mathrm{~nm}$ was observed in samples A and D, but weak or absent in the other samples.

Clearly, sample $\mathrm{E}$ with the zinc to gallium ratio of $\mathrm{Zn}_{1.1} \mathrm{Ga}_{2}$ showed the largest PL response for all excitation wavelengths. Moreover, only the PL using the $313 \mathrm{~nm}$ excitation exhibited the broad 505 emission. Therefore, we used this excitation and $325 \mathrm{~nm}$ excitation from the $\mathrm{HeCd}$ laser to determine 
the relative quantum efficiency of the Zinc gallate powder. As mentioned above, the amount of sample and the weakness of the observed PL using the HgXe illumination source precluded using the integrating sphere for quantum efficiency measurements. Therefore, a comparison was made with the trichromatic sample to get the relative quantum efficiency. These data are shown in Figure 1.87. The relative quantum efficiencies were $1.3 \%$ for $313 \mathrm{~nm}$ illumination and $1.1 \%$ for $325 \mathrm{~nm}$ illumination.
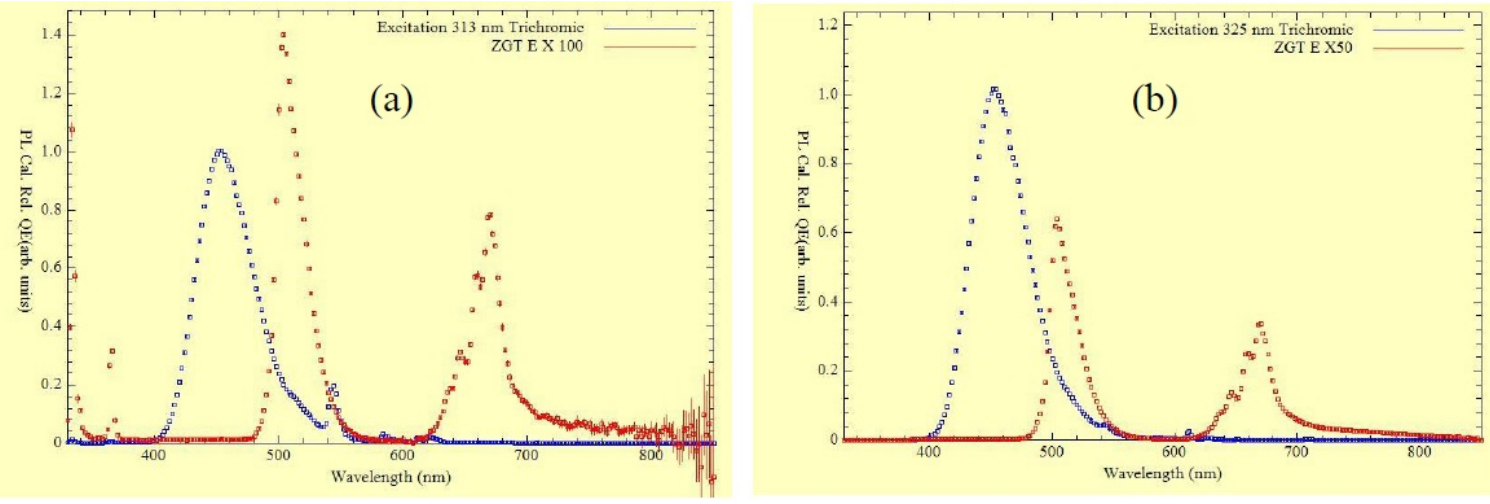

Figure 1.87. Comparison photoluminescence of the trichromatic phosphor and $\mathrm{ZGT131120E}\left(\mathrm{Zn}_{1.1} \mathrm{Ga}_{2}\right)$ using (a) $313 \mathrm{~nm}$ excitation from a HgXe lamp and (b) $325 \mathrm{~nm}$ excitation from the HeCd

Two primary broad transitions at $505 \mathrm{~nm}$ and $670 \mathrm{~nm}$ (probably due to $\mathrm{Mn}^{2+}$ ) were observed in zinc gallate powder made by nanofermentation. The $505 \mathrm{~nm}$ transition was only observed when the sample was illuminated by $313 \mathrm{~nm}$ light, and the intensity of both transitions depended on the sample composition, with the largest photoluminescence occurring for sample $\mathrm{E}\left(\mathrm{Zn}_{1.1} \mathrm{Ga}_{2}\right)$. We also found that some samples showed a very broad photoluminescence at $450-500 \mathrm{~nm}$, probably related to defects in the sample.

For these samples the maximum observed relative QE was 1.3\% for $313 \mathrm{~nm}$ illumination and 1.1\% for $325 \mathrm{~nm}$ illumination. Previous measurements of the relative QE versus excitation energy for ZGT: $\mathrm{Cr}^{3+}$ and AGT:Eu ${ }^{3+}$ showed that the peak excitation occurred for $288 \mathrm{~nm}$ illumination. As a result, it may be possible to get a slightly higher QE at lower wavelengths, but it is uncertain whether the increase would be significant.

The NF technique was developed to synthesize ZGT particles for optoelectronic applications. The wide bandgap of ZGT makes it attractive as host material for dopant induced optical emission control. We were successful in tuning the optical wavelength in the visible part of the spectrum (400-700 nm). However, the optical QE of the samples remained in the range of $\sim 2 \%$ for all the samples indicating inefficient activation of dopants or nanoparticle defect structure dictating the net optical output. The observed low PLQE in ZGT nanoparticles indicates that the more detailed investigation of the particle size effects, surface characteristics, and defect structure is critical to understand the factors dictating the optical and electrical characteristics. A PLQE $>20 \%$ is critical to define a path towards practical applications. The present QE results on ZGT nanoparticles indicate that we need to further evaluate the impact of novel NF process on the particle growth and properties. Binary nanoparticle materials offer a much simpler system than ZGT material to analyze the impact of NF process on the microstructural, optical, and electrical properties of the nanoparticles and thin film coatings.

\subsubsection{Zinc Gallate Annealing and Characterization}

Photoluminescence (PL) measurements were made from different samples of zinc gallate powder as discussed earlier. All the samples were annealed and the impact of nanofermentation process on the 
zinc gallate particles quality was analyzed in terms of the photo-response characteristics. The results of structure-optical properties of Mn doped zinc gallates are summarized in Figure 1.88. As produced zinc gallates are mostly composed of 5-10 nm nanocrystals (Figure 188a,c), showing weak photoluminescence (Figure 188e) that we attribute to the host matrix of zinc gallate (weak bands at $450 \mathrm{~nm}$ and $520 \mathrm{~nm}$ for $350 \mathrm{~nm}$ and $500 \mathrm{~nm}$ excitation respectively). Annealing at $1000^{\circ} \mathrm{C}$ leads to the formation of facetted, large crystals which show strong luminescence from the dopant $\mathrm{Mn}^{2+}$ due to the ${ }^{4} \mathrm{~T}-{ }^{6} \mathrm{~A} 3 \mathrm{~d}$ intrashell transition. [see: S.G. Kim et al., "Optical and Electrical Properties of $\mathrm{ZnGa}_{2} \mathrm{O}_{4} / \mathrm{Mn}^{2+}$ Powder Electroluminescent Device," Materials Letters, 58, 1354 (2004)].
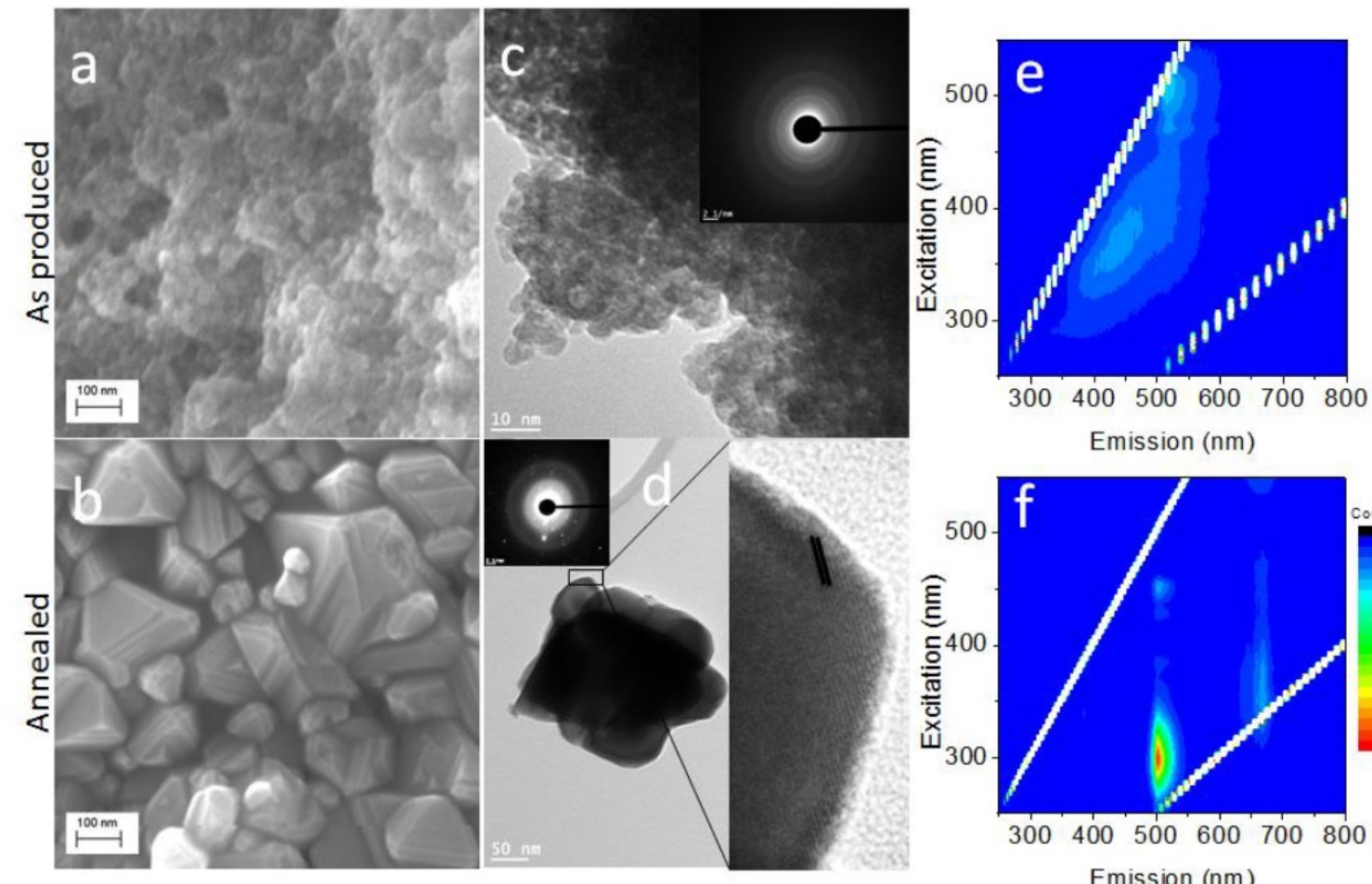

Figure 1.88. Scanning electron micrograph images of zinc gallate.

Scanning electron micrograph images of zinc gallate (a) as produced and (b) after annealing at $1000^{\circ} \mathrm{C}$. Transmission electron micrograph images of (c) as produced zinc gallate showing $(5-10 \mathrm{~nm})$ particles with clear signature of polycrystalline rings in the diffraction pattern (inset) and (d) annealing at $1000^{\circ} \mathrm{C}$ leads to the formation of $>20 \mathrm{~nm}$ crystals with low defects and some polycrystalline/high defect particles of much smaller size, inset of DP and right image showing (111) lattice fringe. Photoluminescence map of (e) as produced and (f) sample annealed at $1000^{\circ} \mathrm{C}$, the weak signature of $\sim 400$ and $520 \mathrm{~nm}$ are common features in both samples while the relative intensity of these peaks is higher for as produced zinc gallate. The annealing leads to the activation of the radiative route as evident by $503 \mathrm{~nm}$ emission (300 nm excitation), which is assigned to the ${ }^{4} \mathrm{~T}-{ }^{6} \mathrm{~A} 3 \mathrm{~d}$ intrashell transition of $\mathrm{Mn}^{2+}$.

\subsubsection{Resistivity measurements of $\mathrm{ZnO}$ films}

This is an important step for the scale up efforts required to produce achieve large amounts of high quality nanofermentation nanoparticles. There is great potential for using nanofermentation to produce nanofermentation nanoparticles for optoelectronics and sensor applications. In order to demonstrate this we have prepared and characterized several $\mathrm{ZnO}$ samples prepared by a PLD. Cross sectional SEM images of PLD-deposited $\mathrm{ZnO}$ films ion $\mathrm{SiO} 2 / \mathrm{Si}$ substrates are shown in Figure 3. We 
achieved the thickness of $\mathrm{ZnO}$ of about $300 \mathrm{~nm}$ and $100 \mathrm{~nm}$ for vacuum and $30 \mathrm{mTorr}_{2}$ deposition conditions Figure 1.89 (a), (b). The in-plane impedance spectra of these films are shown in Figure 1.90). It indicates a transition from pure resistive behavior for frequency range $<10 \mathrm{kHz}$ to capacitive one for higher frequencies for vacuum deposited $\mathrm{ZnO}$ film. Interestingly, the $30 \mathrm{mTorr} \mathrm{ZnO}$ film shows much higher impedance and low frequency resistance, and the transition to capacitive behavior occurs at much higher $(>100 \mathrm{kHz})$ frequency. Initially the samples we prepared using nanofermented $\mathrm{ZnO} \mathrm{NP}$ were characterized by measuring the electrical impedance as a function of frequency. We found that the resistivity of the samples can be reduced to a few $\mathrm{mOhm}-\mathrm{cm}$ therefore providing an excellent opportunity to develop low cost sensors using easily fabricated nanofermented thin film sensors.

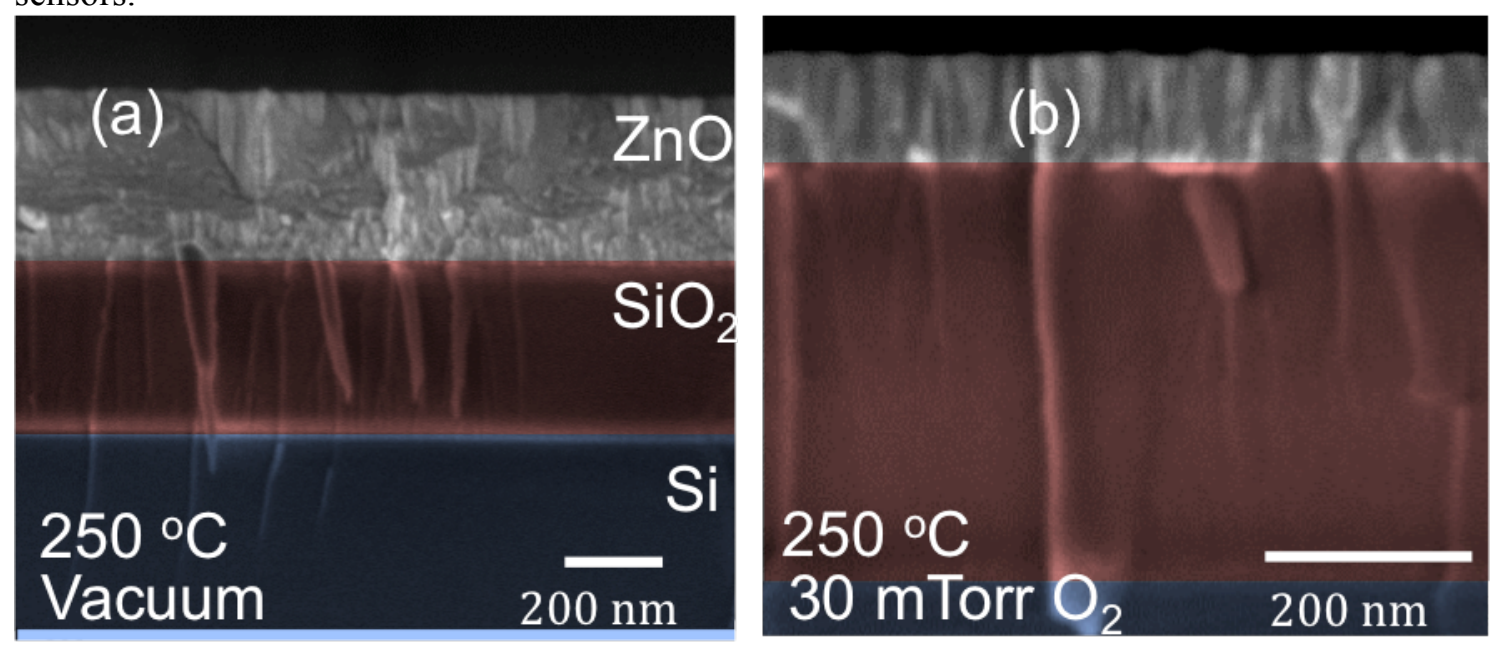

Figure 1.89. Cross sectional SEM images of PLD-deposited $\mathrm{ZnO}$ films ion $\mathrm{SiO}_{2} / \mathrm{Si}$ substrates.

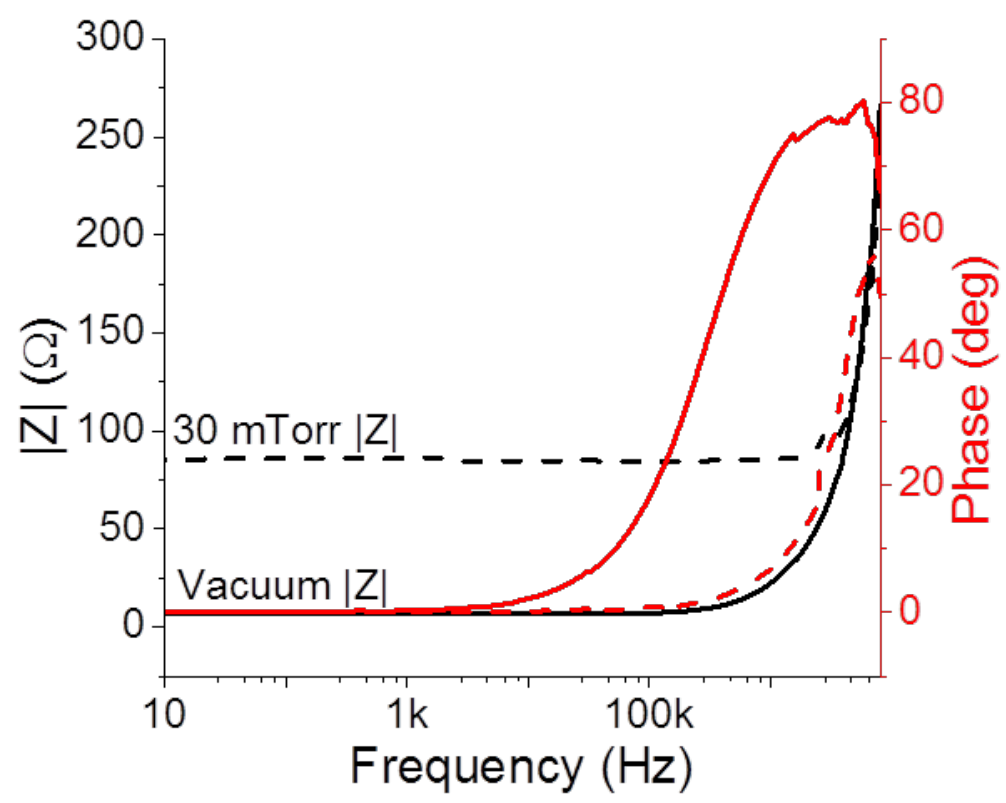

Figure 1.90. In-plane impedance spectra of $\mathrm{PLD}$-deposited $\mathrm{ZnO}$ films ion $\mathrm{SiO}_{2} / \mathrm{Si}$ substrates as a function of frequency.

The different parameters are summarized in Table 1.18 for the PLD-deposited $\mathrm{ZnO}$ films including the thickness, impedance, resistivity and mobility of the $\mathrm{ZnO}$ thin film prepared using pulse laser 
deposition. The nanofermented $\mathrm{ZnO}$ is referred to the film prepared from nanofermented nanoparticles. The samples labeled $\mathrm{ZnO}$ and $\mathrm{Al}-\mathrm{ZnO}$ were commercial samples of pure $\mathrm{ZnO}$ and $\mathrm{ZnO}$ doped with $1 \% \mathrm{Al}$. The lowest resistivity was achieved with intrinsically doped NF NP ZnO (S1) and 1\% Al:ZnO. The resistivity was calculated from the value of the low frequency impedance, thickness of the film and the distance between the electrodes. The value of the resistivity of NFNP film suggests that it can be used for sensing application as well as transparent conducting substrate in PV and LED applications.

Table 1.18 Electrical performance of NF ZnO films prepared using pulse laser deposition

\begin{tabular}{|c|c|c|c|c|c|c|c|c|}
\hline \multirow[b]{2}{*}{ Sample } & \multirow[b]{2}{*}{$\begin{array}{l}\text { Temp } \\
{ }^{\circ} \mathrm{C}\end{array}$} & \multirow[b]{2}{*}{$\mathrm{O}_{2}$ Torr } & \multicolumn{3}{|c|}{ Electrode Dimensions } & \multirow{2}{*}{$\begin{array}{l}\text { Impedanc } \\
\text { e } \Omega\end{array}$} & \multirow{2}{*}{$\begin{array}{l}\text { Resistivity } \\
\Omega \cdot \mathrm{cm}\end{array}$} & \multirow{2}{*}{$\begin{array}{l}\text { Mobility } \\
\mathrm{cm}^{2} / \text { Vs }\end{array}$} \\
\hline & & & $\begin{array}{l}\text { Gap } \\
\mu \mathrm{m}\end{array}$ & $\begin{array}{l}\text { Width } \\
\text { mm }\end{array}$ & $\begin{array}{l}\text { Thickness } \\
\text { nm }\end{array}$ & & & \\
\hline $\begin{array}{l}\text { NF-ZnO } \\
\text { S1 }\end{array}$ & 250 & $\sim 10^{-5}$ & 25 & 2 & 315 & 6.7 & $16.8 \times 10^{-3}$ & 1.04 \\
\hline $\begin{array}{l}\text { NF-ZnO } \\
\text { S2 }\end{array}$ & 250 & $30 \times 10^{-3}$ & 25 & 2 & 88 & 87 & $61.2 \times 10^{-3}$ & 1.3 \\
\hline $\begin{array}{l}\mathrm{Al}-\mathrm{ZnO} \\
\mathrm{S} 1\end{array}$ & 250 & $\sim 10^{-5}$ & 50 & 2 & 220 & 3.3 & $5.8 \times 10^{-3}$ & .003 \\
\hline $\begin{array}{l}\text { Al-ZnO } \\
\text { S4 }\end{array}$ & 250 & $30 \times 10^{-3}$ & 25 & 2 & 362 & 4.3 & $12.5 \times 10^{-3}$ & .009 \\
\hline $\mathrm{ZnO}$ & $400 \mathrm{C}$ & 0.5 & 25 & 2 & 300 & $11.2 \times 10^{6}$ & $26.7 \times 10^{4}$ & \\
\hline
\end{tabular}

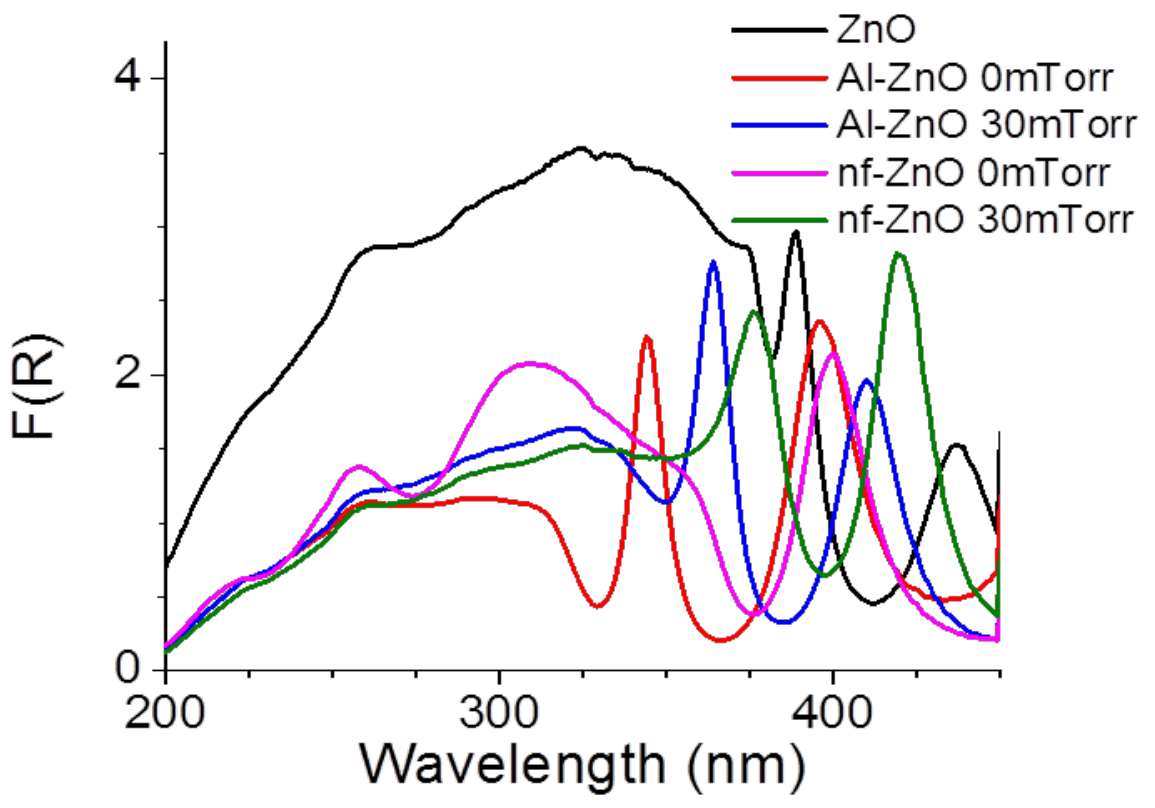

Figure 1.91. UV-Vis diffuse reflectance as a function of wavelength for PLD $\mathrm{ZnO}$ films, the reference and nanofermented deposited on $\mathrm{Si} / \mathrm{SiO}_{2}$ substrate.

Figure 1.91 shows the measured optical properties of PLD ZnO films, and the reference and nanofermented films deposited on $\mathrm{Si} / \mathrm{SiO}_{2}$. The films were measured in a diffuse reflectance configuration. While precise calculation of the optical band gap was obscured by the interference fringes, the estimated the band gap was obtained from the edge of unobscured absorbance. We found that $\mathrm{Al}$ and $\mathrm{NF} \mathrm{ZnO}$ films show upshift in the energy of the band gap, which was also confirmed by 
the band gap energies reported by others, e.g. $(\mathrm{ZnO})=3.31$, e.g. $(\mathrm{Al}: \mathrm{ZnO})=3.4 \mathrm{eV}$ ( see Table 1.19).

Table 1.19 Thin-film Energy Band Gaps

\begin{tabular}{|c|c|}
\hline System & Bandgap Energy (eV) \\
\hline $\mathrm{ZnO}$ & 3.31 \\
\hline $\mathrm{Al}-\mathrm{ZnO}$ & $\sim 3.40$ \\
\hline S-doped $\mathrm{ZnO}$ & $\sim 3.1$ \\
\hline $\mathrm{ZnS}$ & $3.74 \mathrm{eV}$ \\
\hline
\end{tabular}




\subsection{TASK 2: DRYING AND SINTERING OF SILVER INKS}

The main focus of this task was the evaluation of aqueous and solvent based conductive inks developed by NovaCentrix for electronic device applications. The goal was to deposit silver inks on plastic substrates by roll-to-roll compatible inkjet printing and screen-printing techniques. A goal was to use the PulseForge tool to combine the drying and sintering processes into a single pass for high throughput commercial applications - potentially reducing processing time by 2-3 orders of magnitude. The printed metal coating quality and electrical performance was to be evaluated for rollto-roll printed electronic applications.

\subsection{CONDUCTIVE METAL INKS}

The main goal is to identify a rapid, single-pass route to drying and sintering of printed silver inks on flexible plastic substrates using the NovaCentrix PF 3300 pulse thermal processing system. High speed, low cost printing and processing of metal-based inks on low temperature flexible substrates is essential for the advancement of innovative new products in photovoltaics, displays, RFID, sensors, batteries, capacitors, and smart packaging. Because the factors that influence drying and sintering of printed inks place limits on the speed and cost of ink processing and production, a solid understanding of these factors is required. At the same time, ORNL's unique pulse thermal processing technology was exploited to minimize the thermal budget for ink curing, which is a major limitation of the current furnace based annealing approach. Initially, we focused on the curing of silver inks. The main steps in our development and evaluation of conductive metal inks for flexible electronics are highlighted in Figure 2.1.

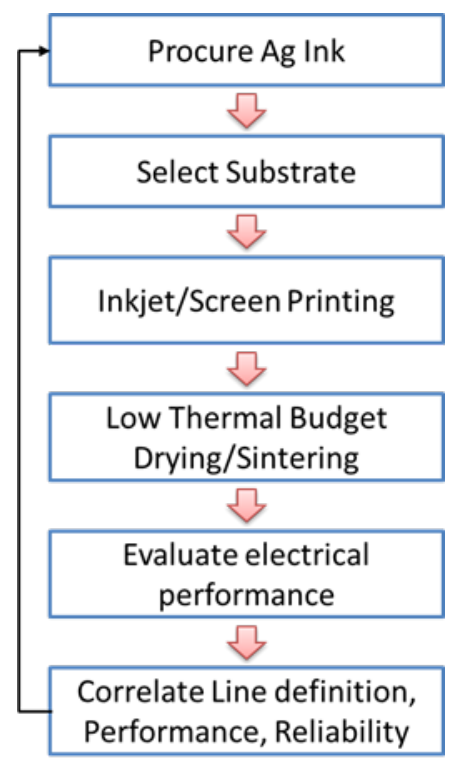

Figure 2.1. General steps in the development and evaluation of the conductive metal inks for flexible electronic applications.

The task encompasses the following focus areas:

- A focus on the evaluation of aqueous and solvent based commercial inks.

- Substrates included porous and non-porous flexible plastics with roll-to-roll potential.

- Printing techniques included inkjet printing and screen printing.

- Sintering techniques included furnace and pulsed thermal processing. 
The initial activities in this task related to the photonic curing of the Ag inks, and were as follows:

- Ink formulations and an experimental matrix were finalized in discussions with NovaCentrix,

- Pulse thermal processing conditions for initial investigation of the simultaneous drying and sintering were identified.

- $\quad$ Substrate materials $(125 \mu \mathrm{m}$ sheet thickness $)$ were identified and acquired.

- $\quad$ Non-porous substrates included PET, ABS, Polycarbonate (PC), Ultem and Kapton.

- $\quad$ Porous substrates used in this study were Novele ${ }^{\mathrm{TM}}$ IJ-220 coated PET.

-

Printable commercial silver inks were identified and acquired.

- Water-based Ag ink: Novacentrix JS-B25HV

- $\quad$ Alcohol-based Ag Ink: Cabot CCI-300

- $\quad$ Equipment to measure the electrical conductance of printed lines were assembled.

Initial efforts to print and furnace process water-based Novacentrix ink were conducted. The results suggest that:

- $\quad$ Substantial drying $(\sim 10-30 \%)$ of the printed lines occurred within minutes of printing. This observation impacted the choice of conditions for subsequent pulsed thermal processing. Coupling the Dimatix printer with the Pulse Forge would be desirable but is presently impractical.

- $\quad$ Lines printed on porous flexible substrates (Novele ${ }^{\mathrm{TM}}$ and paper) are conductive when furnace processed at $\sim 120-160^{\circ} \mathrm{C}$.

Lines printed on non-porous flexible substrates are not conductive when furnace processed at $<\sim 200^{\circ} \mathrm{C}$. This may require ink reformulation.

- $\quad$ Lines are conductive when printed on glass or alumina and processed at $\sim 450^{\circ} \mathrm{C}$.

\subsubsection{Inks on flexible substrates}

Commercial silver conductive inks, both aqueous-based and alcohol-based, were acquired. These inks were successfully printed on several flexible substrates including porous coated PET and nonporous polycarbonate, Ultem, and ABS. Initial attempts to understand the printing, drying and sintering of these inks revealed significant differences in performance. Figure 2.2 shows typical drying results for the alcohol-based silver ink printed on an Ultem substrate. The resistance for similarly printed and furnace dried water-based ink is nearly infinite (non-conductive) for all drying times and temperatures.

The original milestones defined for this project were to achieve a sheet resistance of $<5 \mathrm{~m} \Omega / \mathrm{Sq}$. However, after further consultation with our industry sponsor, NovaCentrix, it was determined that the original milestone was not sufficiently specific. Sheet resistance alone is not sufficient to define the performance of a thin film electrical circuit because the thickness of the printed line is not specified. In order to more accurately define the conductivity of a printed circuit, the industry standard measurements must include both sheet resistance and resistivity, which is typically defined in terms of multiplier of bulk silver. Therefore, the following milestones were proposed and approved for Task 2 during a DOE program review at ORNL on September 11, 2012.

- MS 2.1.2 (6/30/12) Define benchmark oven cure performance for ink-jet printing (Rs < $160 \mathrm{~m} \Omega / \mathrm{Sq}, \sim 3 \mathrm{x}$ bulk Ag) and begin PTP study for ink-jet printed inks

- $\quad$ MS 2.1.3 (9/30/12) Achieve Rs $<210 \mathrm{~m} \Omega / \mathrm{Sq}, \sim 4 \mathrm{x}$ bulk Ag* for ink jet printed materials processed by PTP, initiate study on screen printed materials

- $\quad$ MS 2.1.4 (12/31/12) Achieve Rs $<2.5 \mathrm{~m} \Omega / \mathrm{Sq}, \sim 3 \mathrm{x}$ bulk Ag* for screen printed materials processed by PTP 
- $\quad$ MS $2.2(12 / 31 / 13)$ Demonstrate PTP processed ink jet printed metal lines with Rs $<180$ $\mathrm{m} \Omega / \mathrm{Sq}, \sim 4 \mathrm{x}$ bulk Ag

- MS $2.3(12 / 31 / 14)$ Demonstrate PTP processed ink jet printed metal lines with Rs $<100$ $\mathrm{m} \Omega / \mathrm{Sq}, \sim 1.5 \mathrm{x}$ bulk Ag

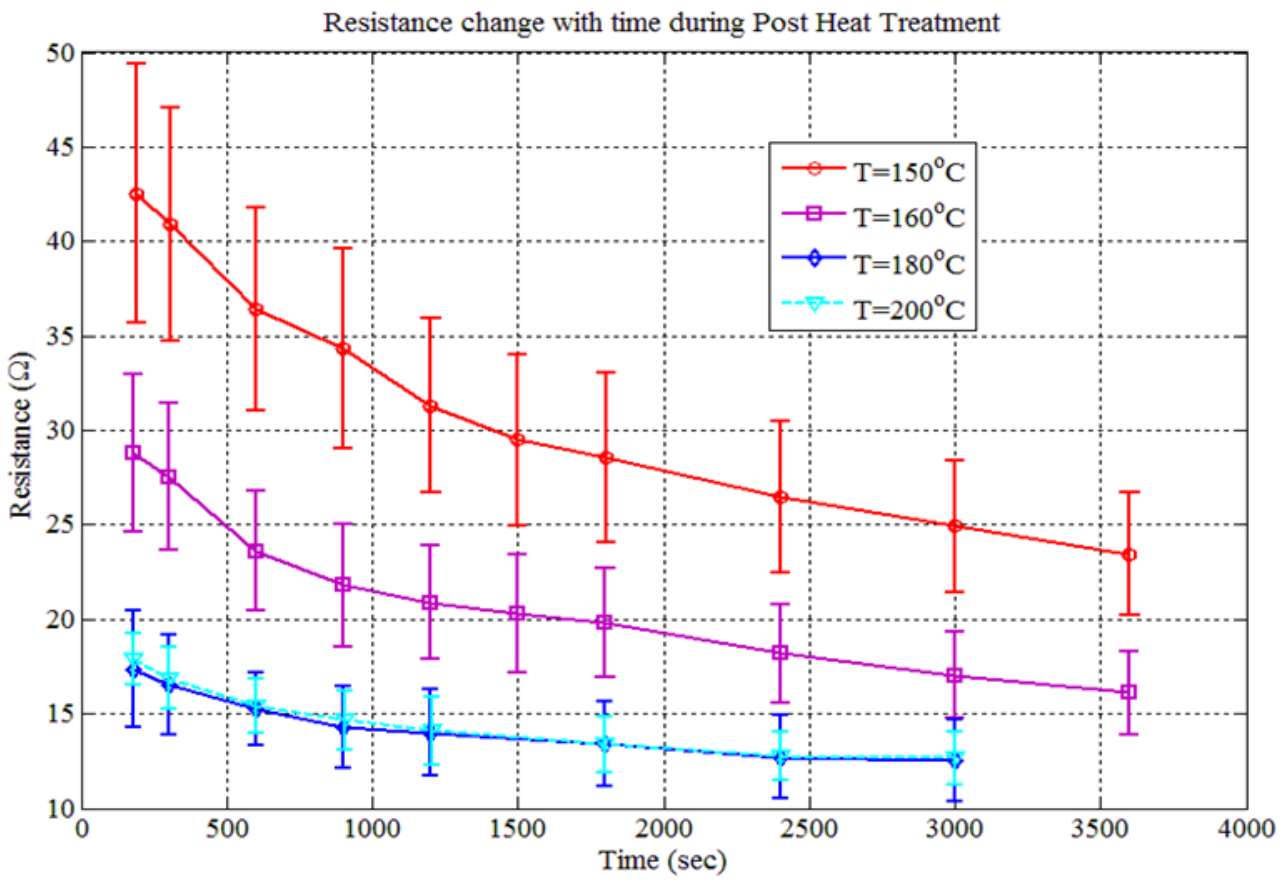

Figure 2.2. Drying results for alcohol-based silver ink on Ultem substrate.

\subsubsection{Sintering and electrical performance of ink jet printed materials}

Evaluation of silver conductive inks subsequently focused on the sintering and electrical performance of ink jet printed materials. Two commercial inks, one water-based and the other alcohol-based, were each inkjet printed on two flexible substrates, one porous (Novele ${ }^{\circledR}$ coated PET) and the other nonporous (Kapton ${ }^{\circledR}$ polyimide).

To establish a baseline for sintering, printed samples for the four combinations of ink and substrate were first furnace sintered in air for various times at a temperature that is compatible with the substrate $\left(150^{\circ} \mathrm{C}\right.$ for PET and $300^{\circ} \mathrm{C}$ for polyimide). The resulting resistivity $(\boldsymbol{\rho})$, sheet resistance $\left(R_{S}\right)$, and minimum furnace sinter time are shown for the four combinations in Table 2.1. Electrical performance was observed to be good, especially for the non-porous Kapton ${ }^{\circledR}$ substrate, but the minimum furnace sintering times are long (5-100 minutes). 
Table 2.1. Results for furnace sintering of inkjet printed silver ink.

\begin{tabular}{|c|c|c|c|c|c|}
\hline Substrate & Ink & $\begin{array}{c}\text { Thickness } \\
\text { ( } \mu \mathrm{m})\end{array}$ & $\begin{array}{l}\text { Furnace } \\
\rho / \rho_{\text {Ag bulk }}\end{array}$ & $\begin{array}{c}\text { Furnace } \\
\mathbf{R}_{\mathbf{s}}(\mathrm{m} \Omega / \square)\end{array}$ & $\begin{array}{c}\text { Furnace } \\
\text { Sinter } \\
\text { Time }\end{array}$ \\
\hline $\begin{array}{l}\text { Novele }^{(\mathbb{R})} \\
\text { (porous) }\end{array}$ & $\begin{array}{c}\text { Cabot } \\
\text { (alcohol) }\end{array}$ & 1.27 & 4.1 & 51 & $5 \mathrm{~min}$ \\
\hline $\begin{array}{l}\text { Novele }^{\circledR} \\
\text { (porous) }\end{array}$ & $\begin{array}{c}\text { Novacentrix } \\
\text { (water) }\end{array}$ & 1.99 & 5.2 & 42 & $10 \mathrm{~min}$ \\
\hline $\begin{array}{c}\text { Kapton }^{\circledR} \\
\text { (non-porous) }\end{array}$ & $\begin{array}{c}\text { Cabot } \\
\text { (alcohol) }\end{array}$ & 1.27 & 1.8 & 22 & $40 \mathrm{~min}$ \\
\hline $\begin{array}{c}\text { Kapton }^{\circledR} \\
\text { (non-porous) }\end{array}$ & $\begin{array}{c}\text { Novacentrix } \\
\text { (water) }\end{array}$ & 1.99 & 1.8 & 14 & $100 \mathrm{~min}$ \\
\hline
\end{tabular}

The electrical performance for PTP sintered samples was generally good except for the alcohol-based Cabot silver ink on Kapton ${ }^{\circledR}$ where ink detachment from the substrate was observed (Table 2.2). The times for PTP sintering (5 - 20 seconds) were, however, substantially shorter than those for furnace sintering (5 - 100 minutes). Short sintering times are clearly desirable for high-rate, low-cost manufacturing processing and were demonstrated here using PTP methods.

Companion samples were sintered in air using a PulseForge 3300 pulsed thermal processing (PTP) tool. Best PTP results for the four sample combinations are shown in Table 2.2.

Table 2.2. Results for PTP sintering of inkjet printed silver ink

\begin{tabular}{|c|c|c|c|c|c|}
\hline Substrate & Ink & $\begin{array}{c}\text { Thickness } \\
\text { ( } \mu \mathrm{m})\end{array}$ & $\begin{array}{c}\text { PTP } \\
\rho / \rho_{\text {Ag bulk }}\end{array}$ & $\begin{array}{c}\text { PTP } \\
\mathbf{R}_{\mathrm{s}}(\mathrm{m} \Omega / \square)\end{array}$ & $\begin{array}{c}\text { PTP } \\
\text { Sinter } \\
\text { Time }\end{array}$ \\
\hline $\begin{array}{l}\text { Novele }^{\circledR} \\
\text { (porous) }\end{array}$ & $\begin{array}{c}\text { Cabot } \\
\text { (alcohol) }\end{array}$ & 1.27 & 1.35 & 17 & $20 \mathrm{sec}$ \\
\hline $\begin{array}{l}\text { Novele }^{\circledR} \\
\text { (porous) }\end{array}$ & $\begin{array}{c}\text { Novacentrix } \\
\text { (water) }\end{array}$ & 1.99 & 3.5 & 25 & $10 \mathrm{sec}$ \\
\hline $\begin{array}{c}\text { Kapton }^{\circledR} \\
\text { (non-porous) }\end{array}$ & $\begin{array}{c}\text { Cabot } \\
\text { (alcohol) }\end{array}$ & 1.27 & detached & detached & detached \\
\hline $\begin{array}{c}\text { Kapton }^{\circledR} \\
\text { (non-porous) }\end{array}$ & $\begin{array}{c}\text { Novacentrix } \\
\text { (water) }\end{array}$ & 1.99 & 6.0 & 50 & $5 \mathrm{sec}$ \\
\hline
\end{tabular}

In the continuing evaluation of silver conductive inks, studies were conducted on screen-printing, furnace and PTP sintering, and the electrical performance of thick silver lines. A commercial, small flake $(\mathrm{D} 50=0.4 \mu \mathrm{m}, \mathrm{D} 90=0.8 \mu \mathrm{m})$, Novacentrix ink was screen-printed using multiple coatings on two flexible substrates, one porous (ArjoWiggins coated paper) and the other non-porous (Kapton ${ }^{\circledR}$ polyimide). Figure 2.3 shows examples of printing for each substrate, and Figure 2.4 show profilometry results after thermal processing. Multiple coatings lead to thicker but generally rougher lines. 

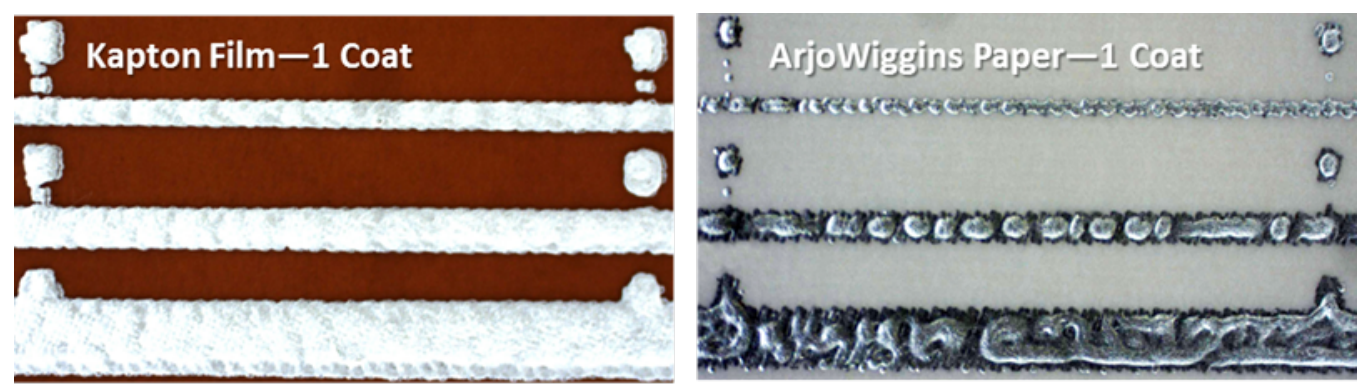

\section{Kapton Film -2 Coats}
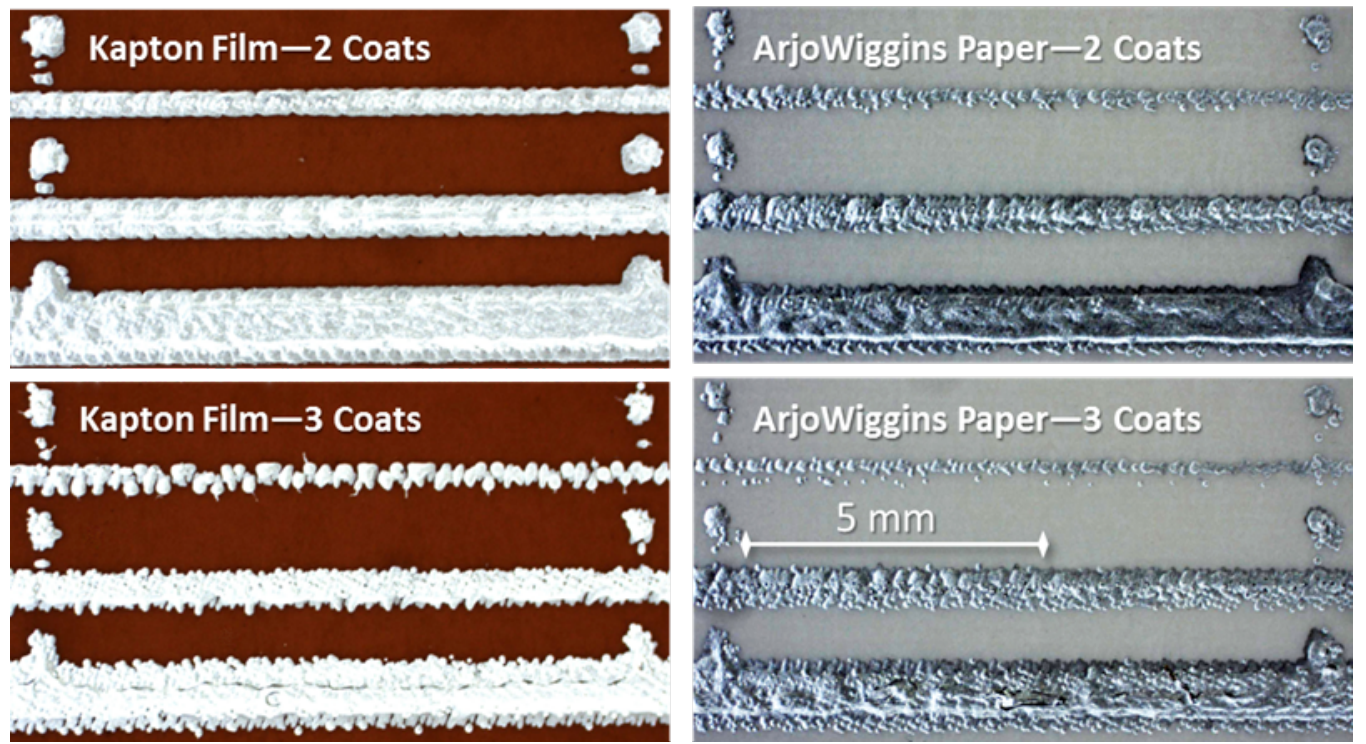

Figure 2.3. Optical micrographs showing typical multi-coat print quality for each substrate.
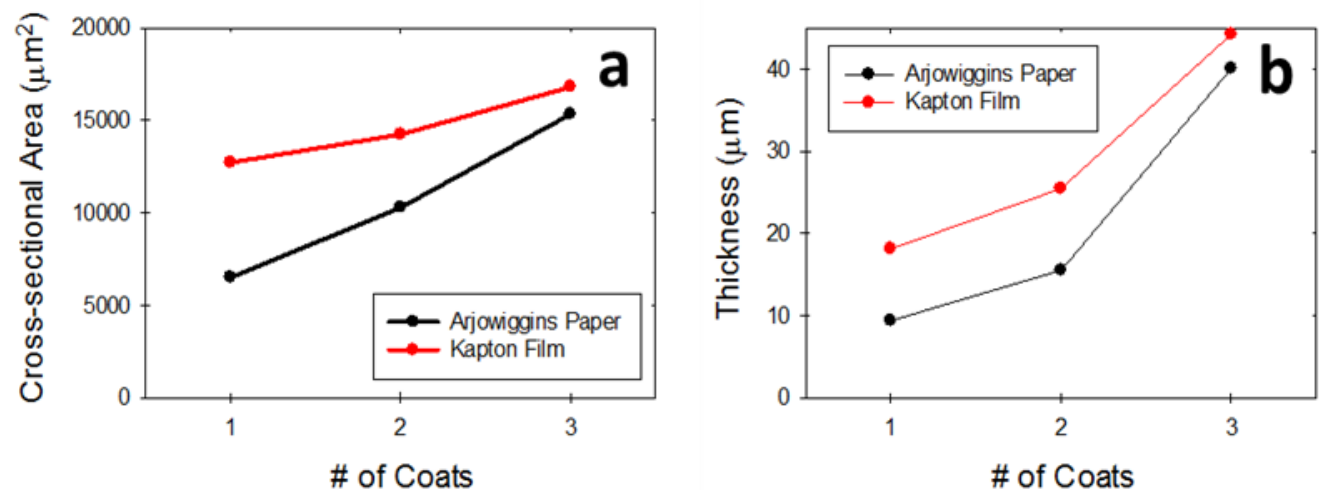

Figure 2.4. Stylus profilometer results showing a) cross-sectional area and b) thickness of sintered multicoat silver lines.

Sintering was initially conducted in air using a PulseForge 3300 pulsed thermal processing (PTP) tool. The best PTP results for the six sample combinations are shown in Table 2.3. Electrical performance after the 8.6 minute PTP processing was generally good, especially for the 2-coat silver on the non-porous Kapton ${ }^{\circledR}$ substrate. 
Table 2.3. Results for PTP and furnace sintering of multi-coat screen printed silver ink.

\begin{tabular}{|c|c|c|c|c|c|}
\hline Substrate & \# of Coats & $\begin{array}{l}\text { Thickness } \\
\qquad(\mu \mathrm{m})\end{array}$ & $\begin{array}{c}\text { Resistivity } \\
\boldsymbol{\rho} / \rho_{\mathrm{Ag} \text { bulk }}\end{array}$ & $\begin{array}{c}\text { Sheet } \\
\text { Resistance } \\
\mathbf{R}_{\mathrm{s}}(\mathrm{m} \Omega / \square)\end{array}$ & $\begin{array}{c}\text { Sinter } \\
\text { Time (min) }\end{array}$ \\
\hline $\begin{array}{l}\text { ArjoWiggins } \\
\text { (porous) }\end{array}$ & 1 & 9.3 & $\begin{array}{c}3.1 \\
(3.0)^{\dagger}\end{array}$ & $\begin{array}{c}5.3 \\
(5.1)^{\dagger}\end{array}$ & $\begin{array}{c}8.6 \\
(249)^{\dagger}\end{array}$ \\
\hline $\begin{array}{l}\text { ArjoWiggins } \\
\text { (porous) }\end{array}$ & 2 & 15.5 & $\begin{array}{c}3.6 \\
(3.1)^{\dagger}\end{array}$ & $\begin{array}{c}3.7 \\
(3.2)^{\dagger}\end{array}$ & $\begin{array}{c}8.6 \\
(249)^{\dagger}\end{array}$ \\
\hline $\begin{array}{l}\text { ArjoWiggins } \\
\text { (porous) }\end{array}$ & 3 & 40.0 & $\begin{array}{c}5.3 \\
(4.3)^{\dagger}\end{array}$ & $\begin{array}{c}2.1 \\
(1.7)^{\dagger}\end{array}$ & $\begin{array}{c}8.6 \\
(249)^{\dagger}\end{array}$ \\
\hline $\begin{array}{c}\text { Kapton }^{\circledR} \\
\text { (non-porous) }\end{array}$ & 1 & 18.1 & $\begin{array}{c}4.3 \\
(3.8)^{\dagger}\end{array}$ & $\begin{array}{c}3.8 \\
(3.3)^{\dagger}\end{array}$ & $\begin{array}{c}8.6 \\
(249)^{\dagger}\end{array}$ \\
\hline $\begin{array}{c}\text { Kapton }^{\circledR} \\
\text { (non-porous) }\end{array}$ & 2 & 25.4 & $\begin{array}{c}2.8 \\
(2.1)^{\dagger}\end{array}$ & $\begin{array}{c}1.7 \\
(1.3)^{\dagger}\end{array}$ & $\begin{array}{c}8.6 \\
(249)^{\dagger}\end{array}$ \\
\hline $\begin{array}{c}\text { Kapton }^{\circledR} \\
\text { (non-porous) }\end{array}$ & 3 & 44.2 & $\begin{array}{c}5.1 \\
(4.0)^{\dagger}\end{array}$ & $\begin{array}{c}1.8 \\
(1.4)^{\dagger}\end{array}$ & $\begin{array}{c}8.6 \\
(249)^{\dagger}\end{array}$ \\
\hline
\end{tabular}

$\dagger$ - PTP followed bv $240 \mathrm{~min} .200^{\circ} \mathrm{C}$ furnace anneal in air.

Following PTP processing, each sample was further furnace sintered in air at $200^{\circ} \mathrm{C}$ for $240 \mathrm{~min}$. For all samples, this additional 240 min furnace treatment improved the electrical performance (see Table 2.4 in parentheses).

The results shown in Table 2.3 demonstrated the capabilities of PTP and screen printing to achieve a sheet resistance of $1.7 \mathrm{~m} \Omega / \mathrm{sq}$ and a resistivity of $2.8 \mathrm{x}$ bulk silver for a $25 \mu \mathrm{m}$ thick silver film on Kapton $^{\circledR}$. The total PTP sintering time in this case was only 8.6 minutes. Additional furnace annealing in air at $200^{\circ} \mathrm{C}$ for 240 minutes led to further improvements in both sheet resistance and resistivity of nearly $25 \%$.

In further studies, two commercial Novacentrix inks, one large flake $(\mathrm{D} 50=2 \mu \mathrm{m} ; \mathrm{D} 90=4 \mu \mathrm{m})$ and the other small flake $(\mathrm{D} 50=0.4 \mu \mathrm{m}, \mathrm{D} 90=0.8 \mu \mathrm{m})$, were each screen printed on two flexible substrates, one porous (ArjoWiggins coated paper) and the other non-porous (Kapton ${ }^{\circledR}$ polyimide). Inks were printed using an AMI screen printer and a steel wire (0.0011" diameter) screen with a 230/inch mesh count and a 0.0014 " emulsion thickness. Lines of ink were printed in widths ranging from $25 \mu \mathrm{m}$ to $1 \mathrm{~mm}$. Figure 2.5 shows examples of printing for each ink/substrate combination. 

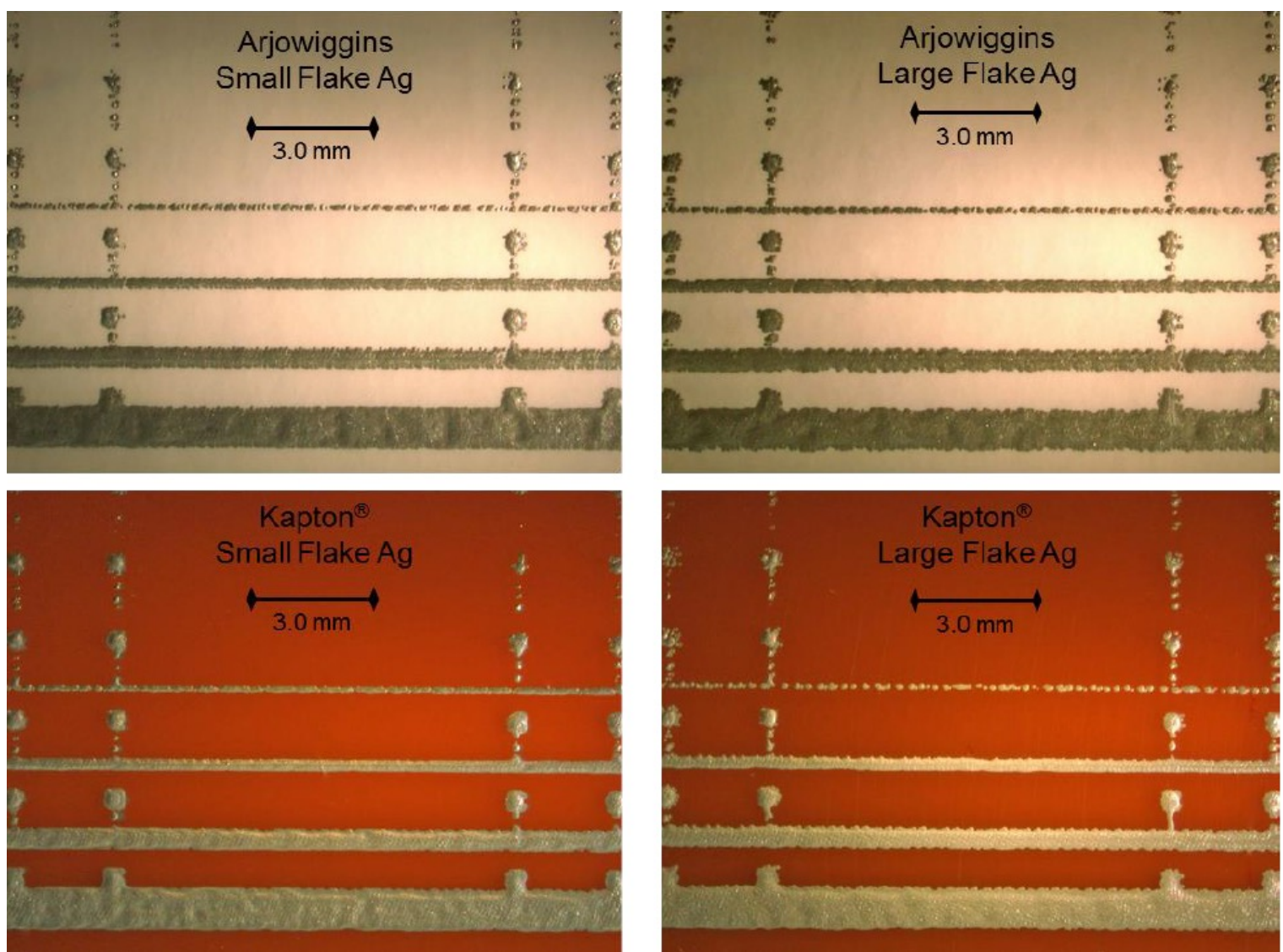

Figure 2.5. Optical micrographs showing typical print quality for each ink/substrate combination.

To establish a baseline for sintering, screen printed samples for the four combinations of ink and substrate were first furnace sintered in air for various times at a temperature that is compatible with both substrates $\left(200^{\circ} \mathrm{C}\right)$. The resulting resistivity $(\boldsymbol{\rho})$, sheet resistance $(\mathrm{Rs})$, and minimum furnace sinter time are shown for the four combinations in Table 2.4. Electrical performance was found to be good, especially for the non-porous Kapton ${ }^{\circledR}$ substrate, but the furnace sintering times were long (240 minutes).

Table 2.4. Results for furnace sintering of screen printed silver ink.

\begin{tabular}{|c|c|c|c|c|c|}
\hline Substrate & Ink & $\begin{array}{c}\text { Thickness } \\
\text { ( } \mu \mathrm{m})\end{array}$ & $\begin{array}{l}\text { Furnace } \\
\rho / \rho_{\text {Ag bulk }}\end{array}$ & $\begin{array}{c}\text { Furnace } \\
\mathbf{R}_{\mathbf{s}}(\mathrm{m} \Omega / \square)\end{array}$ & $\begin{array}{c}\text { Furnace } \\
\text { Sinter } \\
\text { Time }\end{array}$ \\
\hline $\begin{array}{l}\text { ArjoWiggins } \\
\text { (porous) }\end{array}$ & $\begin{array}{l}\text { Large Flake } \\
\text { Silver }\end{array}$ & 15.8 & 5.3 & 5.3 & $240 \mathrm{~min}$ \\
\hline $\begin{array}{l}\text { ArjoWiggins } \\
\text { (porous) }\end{array}$ & $\begin{array}{l}\text { Small Flake } \\
\text { Silver }\end{array}$ & 18.7 & 3.8 & 3.3 & $240 \mathrm{~min}$ \\
\hline $\begin{array}{c}\text { Kapton }^{\circledR} \\
\text { (non-porous) }\end{array}$ & $\begin{array}{l}\text { Large Flake } \\
\text { Silver }\end{array}$ & 18.0 & 4.0 & 3.5 & $240 \mathrm{~min}$ \\
\hline $\begin{array}{c}\text { Kapton }^{\circledR} \\
\text { (non-porous) }\end{array}$ & $\begin{array}{l}\text { Small Flake } \\
\text { Silver }\end{array}$ & 13.1 & 3.0 & 3.6 & $240 \mathrm{~min}$ \\
\hline
\end{tabular}

Companion samples were then sintered in air using a PulseForge 3300 pulsed thermal processing 
(PTP) tool. Best PTP results for the four sample combinations are shown in Table 2.5.

Table 2.5. Results for PTP sintering of screen printed silver ink

\begin{tabular}{|c|c|c|c|c|c|}
\hline Substrate & Ink & $\begin{array}{l}\text { Thickness } \\
\qquad(\mu \mathrm{m})\end{array}$ & $\begin{array}{l}\text { Furnace } \\
\rho / \rho_{\text {Ag bulk }}\end{array}$ & $\begin{array}{c}\text { PTP } \\
\mathbf{R}_{\mathrm{s}}(\mathbf{m} \Omega / \square)\end{array}$ & $\begin{array}{l}\text { PTP } \\
\text { Sinter } \\
\text { Time }\end{array}$ \\
\hline $\begin{array}{l}\text { ArjoWiggins } \\
\text { (porous) }\end{array}$ & $\begin{array}{l}\text { Large Flake } \\
\text { Silver }\end{array}$ & 15.8 & $\begin{array}{c}8.2 \\
\left(3.1^{\dagger}\right)\end{array}$ & $\begin{array}{c}8.2 \\
\left(3.1^{\dagger}\right)\end{array}$ & $\begin{array}{c}8.6 \min \\
\left(249 \min ^{\dagger}\right)\end{array}$ \\
\hline $\begin{array}{l}\text { ArjoWiggins } \\
\text { (porous) }\end{array}$ & $\begin{array}{l}\text { Small Flake } \\
\text { Silver }\end{array}$ & 18.7 & 13.7 & 11.7 & $8.6 \mathrm{~min}$ \\
\hline $\begin{array}{c}\text { Kapton }^{\circledR} \\
\text { (non-porous) }\end{array}$ & $\begin{array}{l}\text { Large Flake } \\
\text { Silver }\end{array}$ & 18.0 & $\begin{array}{c}5.6 \\
\left(3.6^{\dagger}\right)\end{array}$ & $\begin{array}{c}4.9 \\
\left(3.1^{\dagger}\right)\end{array}$ & $\begin{array}{c}8.6 \min \\
\left(249 \min ^{\dagger}\right)\end{array}$ \\
\hline $\begin{array}{c}\text { Kapton }^{\mathbb{B}} \\
\text { (non-porous) }\end{array}$ & $\begin{array}{l}\text { Small Flake } \\
\text { Silver }\end{array}$ & 13.1 & 3.1 & 3.7 & $8.6 \mathrm{~min}$ \\
\hline
\end{tabular}

$\dagger$ - PTP sintered followed by $240 \mathrm{~min}, 200^{\circ} \mathrm{C}$ furnace anneal.

The electrical performance for PTP sintered samples was also good except for the large flake silver ink on ArjoWiggins paper. This performance could likely have been improved with a subsequent 240 min, $200^{\circ} \mathrm{C}$ furnace anneal. Although the electrical performance of the PTP-only sintered samples are generally not as good as that for their furnace sintered companions, the times for PTP-only sintering ( 8.6 minutes) are substantially shorter than those for furnace sintering ( 240 minutes). The milestone selected as the goal for PTP samples was to achieve a sheet resistance $<2.5 \mathrm{~m} \Omega / \mathrm{Sq}$ at approximately $3 \mathrm{x}$ bulk Ag for screen printed materials. Although we were able to achieve a high quality for the printed material (3x bulk Ag conductivity), the goal of $<2.5 \mathrm{~m} \Omega / \mathrm{Sq}$ was not met during these studies. The thickness of the screen printed lines could be increased significantly in order to meet this metric by adjusting various print parameters.

\section{Kapton ${ }^{\circledR}$ plastic substrate ArjoWiggins paper substrate}

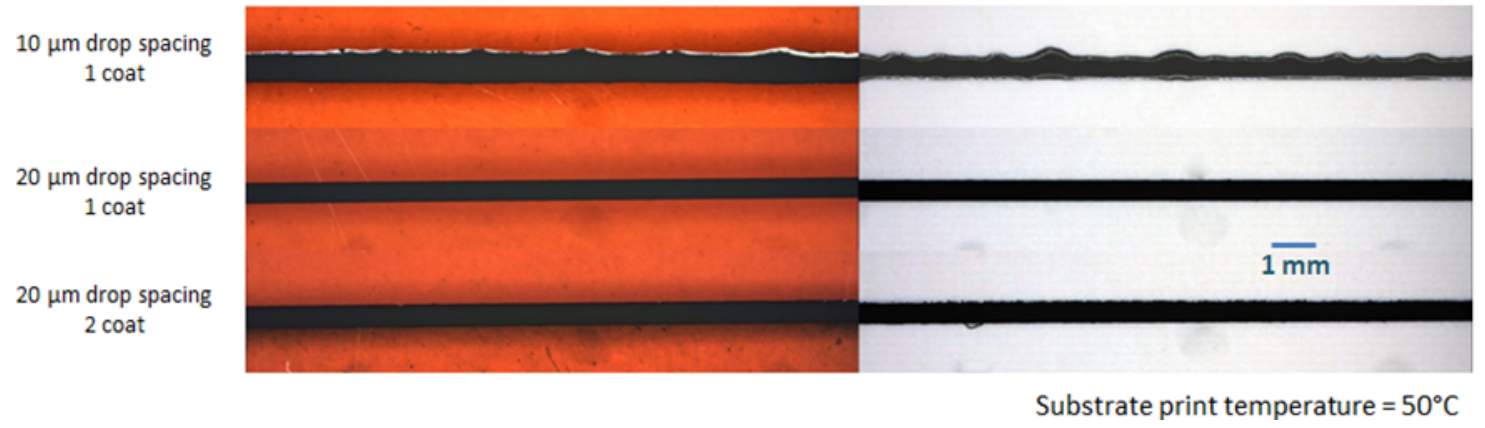

Figure 2.6. Optical micrographs showing typical print quality for each substrate.

To demonstrate the reduced resistance of increased line thickness, a commercial solvent based silver ink (Cabot) was printed using single and multiple coatings on two flexible substrates, one paper-based (ArjoWiggins coated paper) and the other polymer-based (Kapton ${ }^{\circledR}$ polyimide). Figure 2.6 shows examples of printing for each substrate. After printing, the substrates were furnace sintered at $200^{\circ} \mathrm{C}$ for 240 minutes. Figure 2.7 shows the results for the printed lines after printing and furnace sintering. In all cases the electrical resistance of the silver line printed on the paper-based substrate was less than the corresponding line on the polymer-based substrate. 


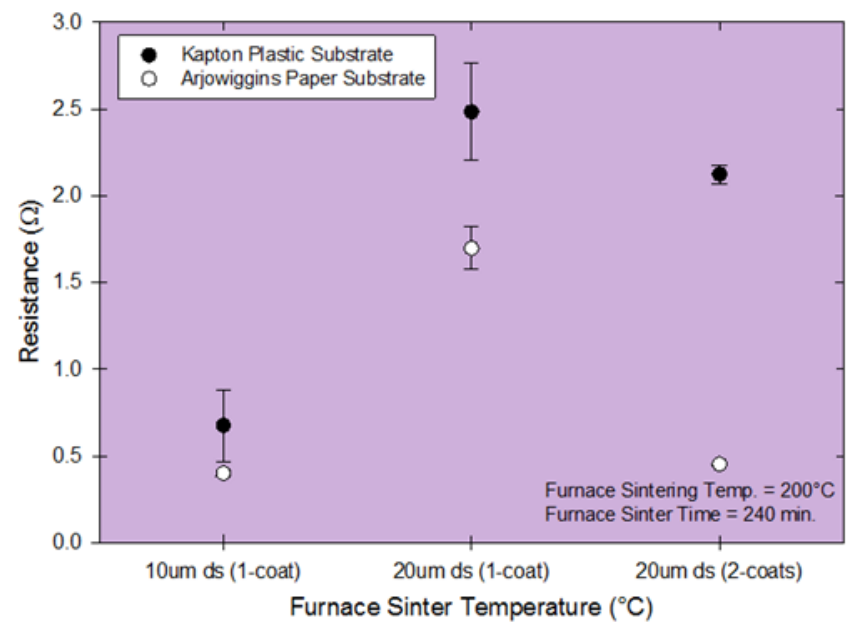

Figure 2.7. Electrical resistance for paper- and polymer-based substrates.

Table 2.6 shows a summary of the electrical results.

Table 2.6. Results for inkjet printing and furnace sintering on paper and polymer substrates

\begin{tabular}{|c|c|c|c|c|c|c|}
\hline Sample & $\mathrm{R}(\Omega)$ & $\mathrm{A}\left(\mathrm{cm}^{2}\right)$ & $\rho(\mu \Omega \cdot \mathrm{cm})$ & $\rho / \rho \mathrm{Ag}$ & $\mathrm{t}(\mu \mathrm{m})$ & $\mathrm{Rs}(\mathrm{m} \Omega / \mathrm{sq})$ \\
\hline Kapton $10 \mu \mathrm{m}$ ds 1-c \#3 & 0.6757 & $1.371 \times 10-5$ & 9.26 & 5.82 & 1.55 & 59.7 \\
\hline Kapton $20 \mu \mathrm{m}$ ds 1-c \#5 & 2.4840 & $4.18 \times 10-6$ & 10.4 & 6.54 & 0.735 & 141.5 \\
\hline Kapton $20 \mu \mathrm{m}$ ds 2-c \#7 & 2.1240 & $5.34 \times 10-6$ & 11.5 & 7.23 & 0.818 & 140.5 \\
\hline Arjo $10 \mu \mathrm{m}$ ds 1-c \#4 & 0.4000 & $1.371 \times 10-5$ & 5.48 & 3.45 & 1.55 & 35.4 \\
\hline Ario 20 $\mu \mathrm{m}$ ds 1-c \#6 & 1.6980 & $4.18 \times 10-6$ & 7.10 & 4.47 & 0.735 & 96.6 \\
\hline Ario 20 $\mu \mathrm{m}$ ds 2-c \#8 & 0.4522 & $5.34 \times 10-6$ & 2.41 & 1.52 & 0.818 & 29.5 \\
\hline
\end{tabular}

The next task in demonstrating printable metal inks was to develop a printable insulating coating. The chosen ink was a Sun Chemical polymer-based ink, which required UV light for both setting and curing. UV setting must occur within seconds of printing to prevent the ink from spreading on the substrate. This was accomplished with the light guide of a UV source attached to the inkjet print head. The light guide was offset a distance from the ink nozzles to avoid UV exposure of the ink at the nozzles.

Figure 2.8 shows an example of a test print pattern for UV setting of ink. The two $5 \times 5$ arrays of squares are offset a distance equal to the distance between the UV light guide and the print nozzles. As the print head travels for left to right, first a portion of the left array is printed and then a portion of the right array is printed. While the right array is printed, the UV light guide passes over the left array. If the UV intensity and print speed are correct, the final result is a UV-set left array and an unset right array. 

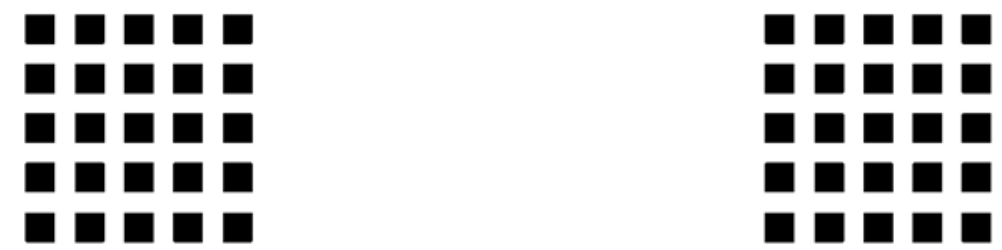

Figure 2.8. Two 5 x 5 arrays of printed squares.

The UV setting polymer was successfully printed and cured on both non-porous glass and flexible PET substrates. Controlling the intensity of the UV exposure varied the smoothness of the top surface of the cured polymer. Figure 2.9 shows the surface profile of six lines of polymer that were cured using different UV intensities. The surface smoothness increases monotonically with decreasing UV intensity.

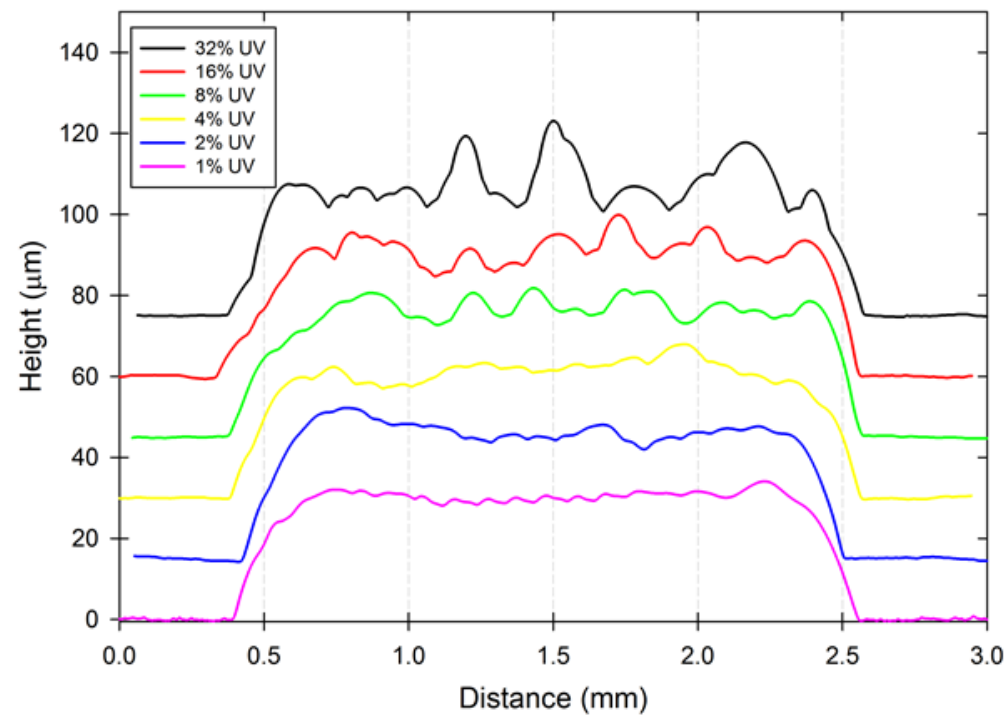

Figure 2.9. Surface profile of six lines cured using different UV light intensities.

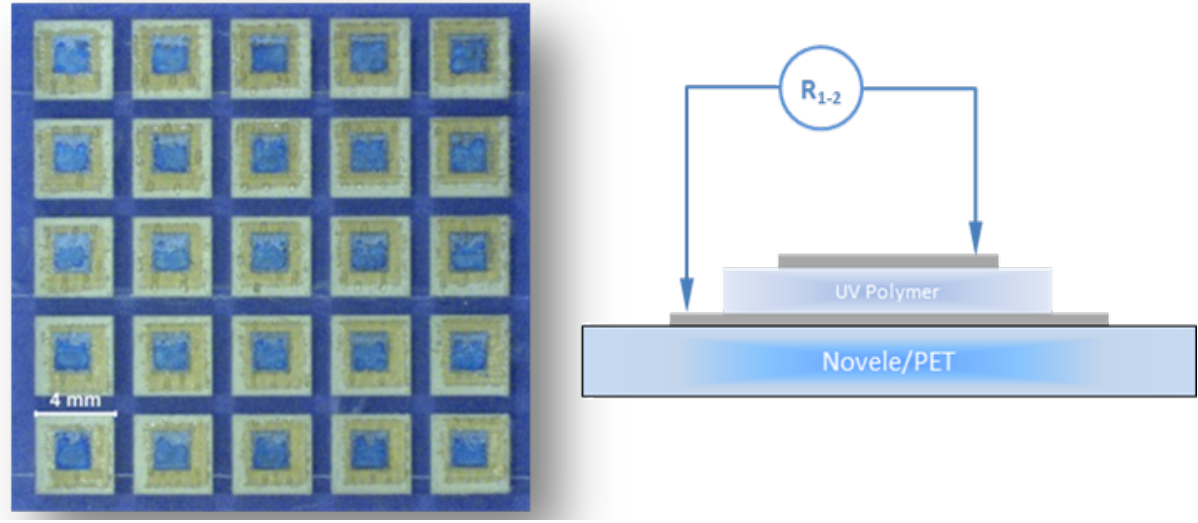

Figure 2.10. Example of a 5 x 5 array of 3-layer printed stacks.

A layer of UV polymer was printed between two layers of printed silver on a PET substrate to form a 3-layer printed stack (see Figure 2.10). A total of fifty such stacks were fabricated and electrically 
tested. Low resistance for some of the stacks was attributable to pinholes in the polymer layer or printer misalignment.

The structures discussed above consisted of alternating layers of printed silver and printed UV setting polymer. The UV setting intensity for these structures was chosen to be $32 \%$, and more than half of the printed structures exhibited low resistance (i.e., poor performance) that was attributed to either pinholes in the printed polymer or printer misalignment. To address the problem of pinholes, the intensity of the UV exposure was reduced from the $32 \%$ to $1 \%$ to allow the polymer ink more time to flow prior to being "set". Figure 2.11 shows a substantially smoother surface for the polymer cured at $2 \%$ than at $32 \%$. Twenty-five additional multi-layer structures were printed using $2 \%$ UV intensity. Only $20 \%$ of these structures showed low resistance. This result suggests that control of the UV setting intensity reduces pinholes in the polymer and improves the performance of the multilayer structure.

\section{$32 \%$ UV}

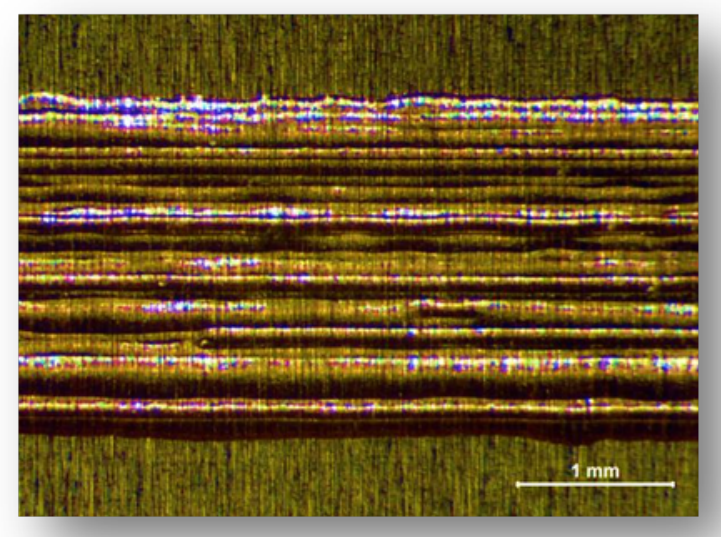

\section{$2 \%$ UV}

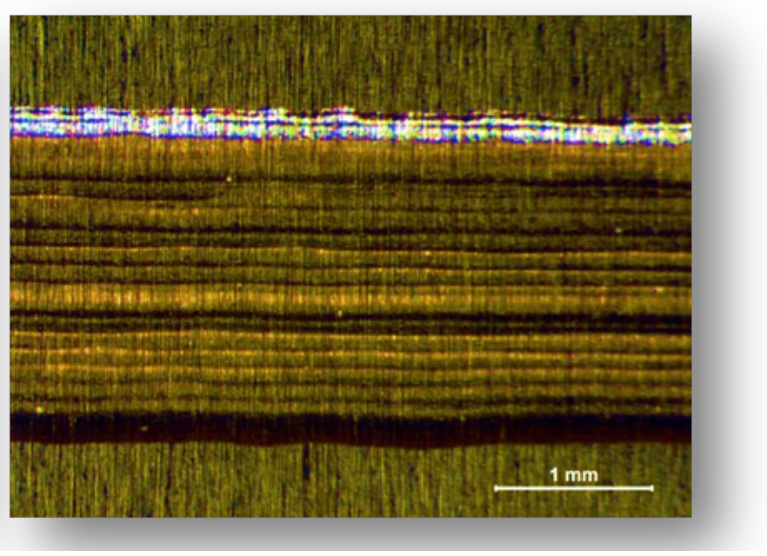

Figure 2.11. Images of a silver strain gauge printed directly on the FDM photopolymer substrate. The polymer was printed using $U V$ intensities $32 \%$ and $2 \%$.

A simple resistive strain gauge was designed for inkjet printing on Fused Deposition Modeling (FDM) substrates. A Stratasys Objet 30 Pro 3D printer was chosen to prepare the $\sim 25 \times 25 \mathrm{~mm}$ FDM substrates using a black rigid UV-curable photopolymer (VeroBlack Plus ${ }^{\mathrm{TM}}$ ). The Objet printing process includes a surface rolling/pressing step that tends to improve interlayer bonding, surface density, and surface smoothness. The smoother denser surface of the Objet substrate reduces some of the challenges associated with printing of conductors on FDM surfaces.

Figure 2.12 is an image of a typical silver strain gauge printed directly on the FDM photopolymer substrate. The higher magnification inset (upper right) shows the quality and fine features achievable for the $\sim 250 \mu \mathrm{m}$ wide printed silver lines. When printed directly on the photopolymer substrate, the strain gauge exhibited a resistance as low as $\sim 50 \Omega$. To successfully demonstrate an embedded printed strain gauge within an FDM structure, the printed strain gauge (above) needs to be overlaid with FDM material and tested. Testing would include circuit resistance, gauge factor, and tensile strength of the FDM structure. 


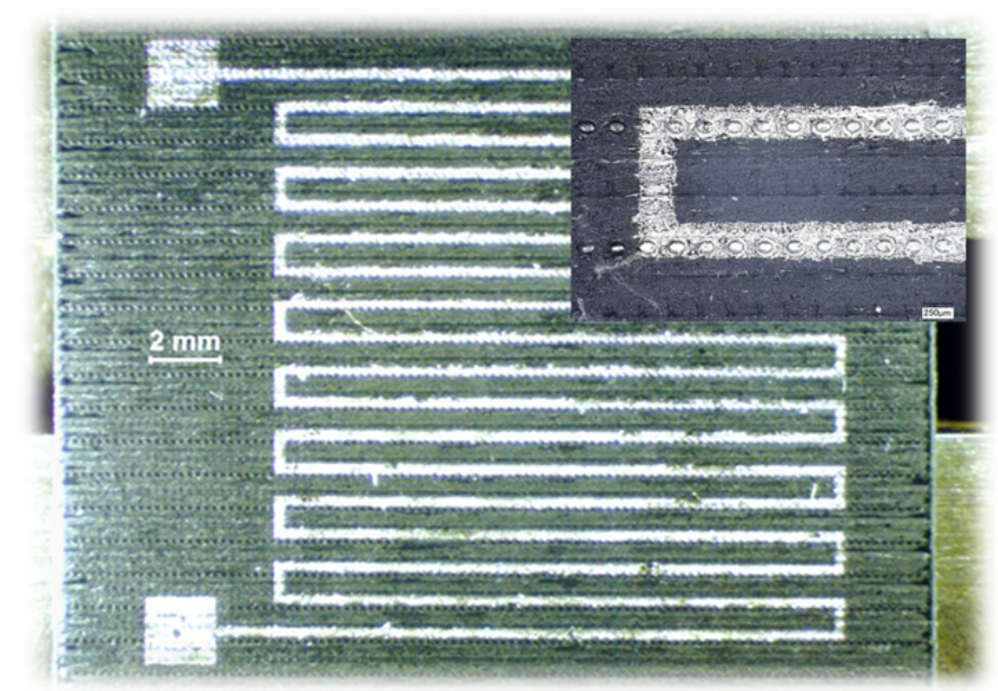

Figure 2.12. Silver resistive strain gauge printed on an FDM substrate. Inset shows higher magnification of the printed line.

The continuing work on this task next focused on curing the inkjet silver nanoparticles using photon pulses. The inks were from different sources (NovaCentrix and Cabot). Two different print thicknesses were used (i.e. $1.99 \mu \mathrm{m}$ and $1.27 \mu \mathrm{m}$ ) on two different substrates. The sintering time was varied from $5 \mathrm{~s}$ to $10 \mathrm{~s}$ to $20 \mathrm{~s}$. In Table 2.7 we summarize the details for the curing process and the measured resistance. For the polyimide substrate, we observed in one case detachment of the ink layer. In Figure 2.13 we plotted the measured resistance for the four samples described in Table 2.6 as a function of sintering time. In Figure 2.14 we show the effect of the pulse frequency on the measured resistance as a function of sintering time for the four samples described in Table 2.6.

Table 2.7. Pulsed photonic curing of inkjet silver nanoparticles

\begin{tabular}{|l|l|l|l|l|l|l|l|l|} 
Substrate & \multicolumn{1}{|c|}{ Ink } & $\begin{array}{c}\text { Thickness } \\
(\boldsymbol{\mu m})\end{array}$ & $\begin{array}{c}\text { Furnace } \\
\boldsymbol{\rho} / \rho_{\text {bulk }}\end{array}$ & $\begin{array}{c}\text { Furnace } \\
\mathbf{R}_{\mathbf{s}} \\
(\mathbf{m} \mathbf{\Omega} / \mathbf{s q})\end{array}$ & $\begin{array}{c}\text { Furnace } \\
\text { Sinter } \\
\text { Time }\end{array}$ & $\begin{array}{c}\text { PPC } \\
\boldsymbol{\rho} / \rho_{\text {bulk }}\end{array}$ & $\begin{array}{c}\text { PPC } \\
\mathbf{R}_{\mathbf{s}} \\
(\mathbf{m} \mathbf{\Omega} / \mathbf{s q})\end{array}$ & $\begin{array}{c}\text { PPC } \\
\text { Sinter } \\
\text { Time }\end{array}$ \\
\hline $\begin{array}{l}\text { Oxide } \\
\text { coated PET }\end{array}$ & Novacentrix & 1.99 & 5.2 & 42 & $10 \mathrm{~min}$ & 3.5 & 25 & $10 \mathrm{sec}$ \\
\hline $\begin{array}{l}\text { Oxide } \\
\text { coated PET }\end{array}$ & Cabot & 1.27 & 4.1 & 51 & $5 \mathrm{~min}$ & 1.35 & 17 & $20 \mathrm{sec}$ \\
\hline Polyimide & Novacentrix & 1.99 & 1.8 & 14 & $100 \mathrm{~min}$ & 6.0 & 50 & $5 \mathrm{sec}$ \\
\hline Polyimide & Cabot & 1.27 & 1.8 & 22 & $40 \mathrm{~min}$ & detached & detached & detached \\
\hline
\end{tabular}



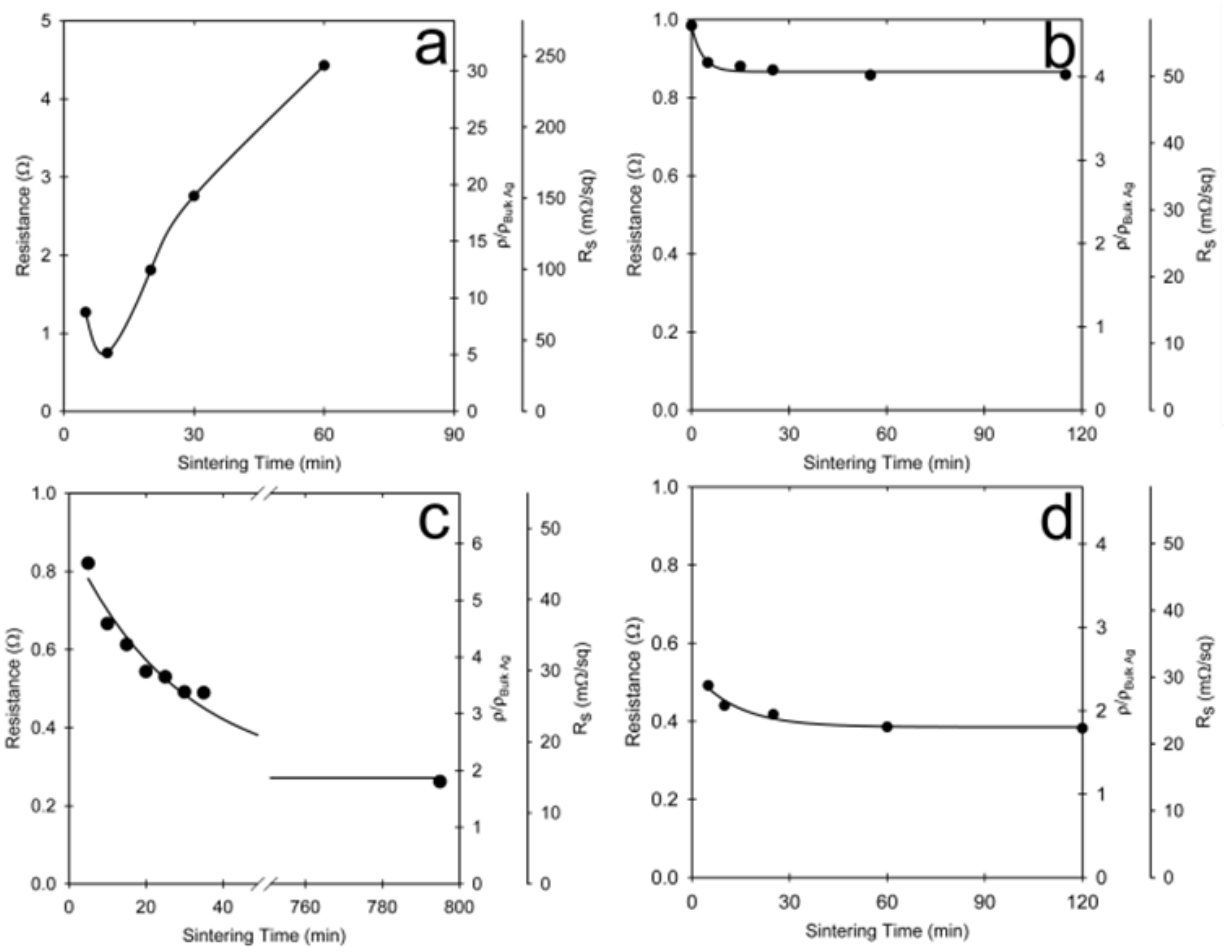

Figure 2.13. Resistance as a function of sintering time for the cured inks.
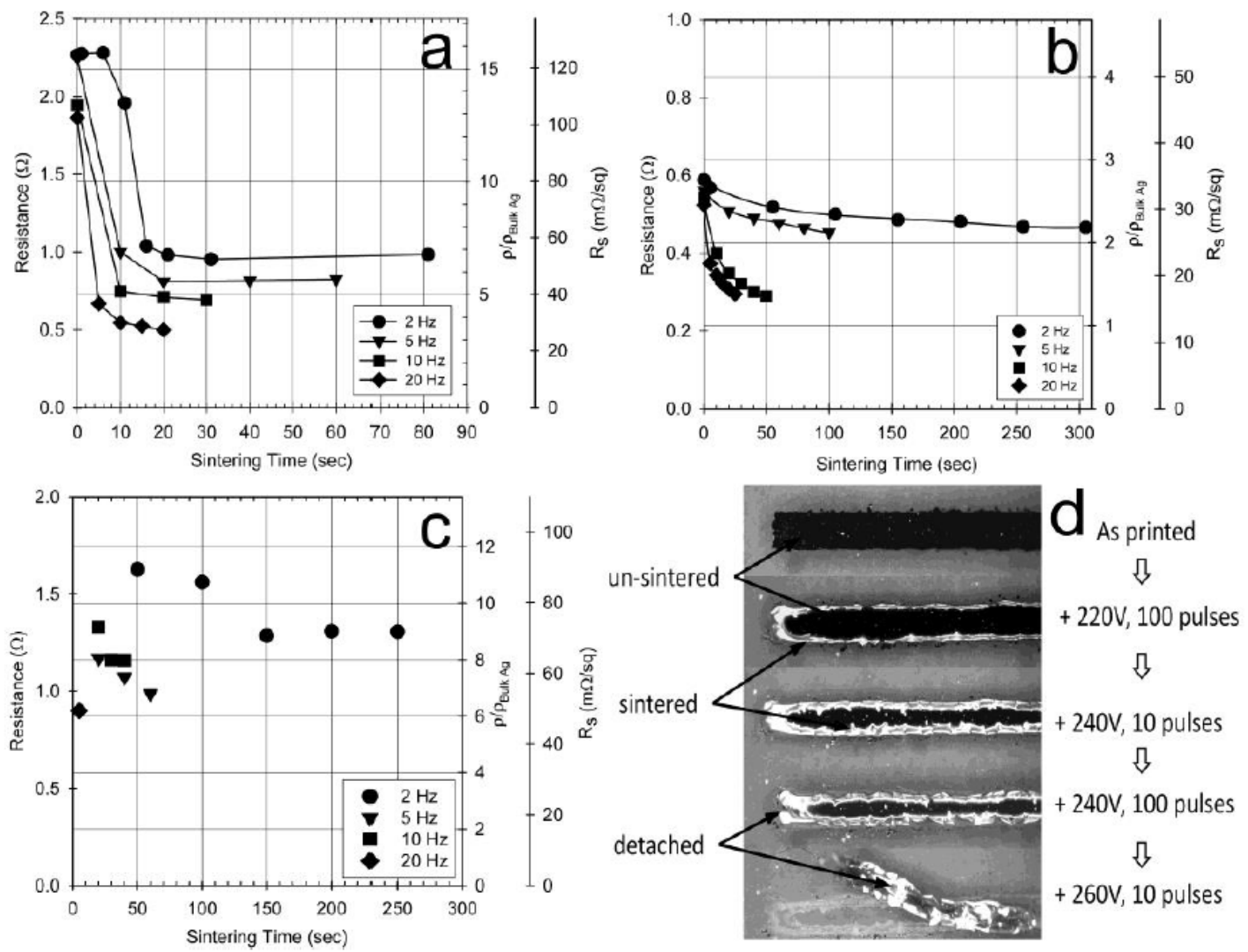

Figure 2.14. Resistance as a function of sintering time and pulse frequency rate for the cured inks. 
In further studies we used inks to silver print FDM substrates with 1-mm thick FDM material. The FDM frame and printed substrate were used to fabricate an embedded strain gauge, and to successfully embed a printed strain gauge within a FDM structure using a frame that was fixed to the print bed of the Stratasys Objet 30 Pro 3D printer. Figure 2.15 shows a photograph of a prototype embedded strain gauge with the inkjet printed silver conducting line embedded between two 1-mm thick FDM layers.

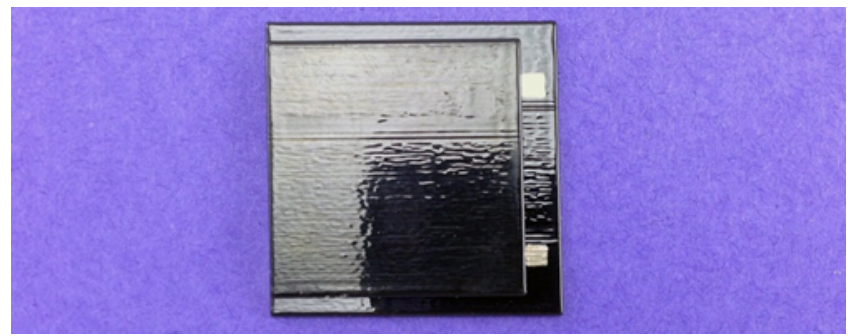

Figure 2.15. A prototype embedded strain gauge. Inkjet printed silver between two 1-mm thick FDM layers.

The process of embedding circuitry in the printed structure involved three distinct steps. In the first step, a Dimatix printer was used to print the conductive traces on the substrate. The second step involved embedding the traces while printing sockets for components using the Stratasys Objet 30 Pro 3D printer. In the third and final step, the components were inserted and overprinted using the Stratasys Objet 30 Pro 3D printer. Figure 2.16 shows schematically the three-step process.

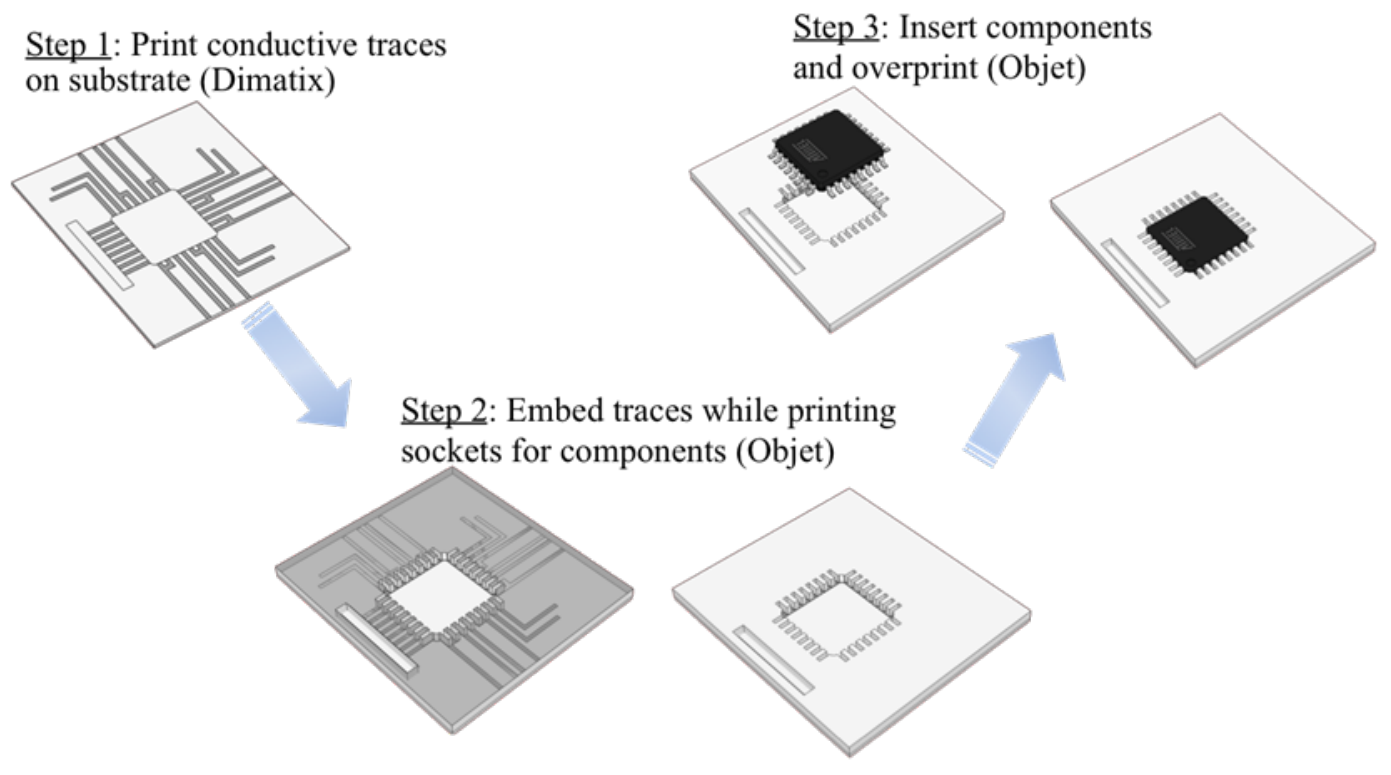

Figure 2.16. The three step process used to embed circuitry in printed structures.

One of the significant efforts in this project was to embed complex circuit geometries that would provide additional functionality at a reduced cost (see Figure 2.17). For example, implementing Arduino complex logic circuitry and incorporating a surface-mount microchip with various surface mount components such as LEDs, capacitors, resistors, memory and display would provide unprecedented functionality and control using roll-to-roll printable electronics. 


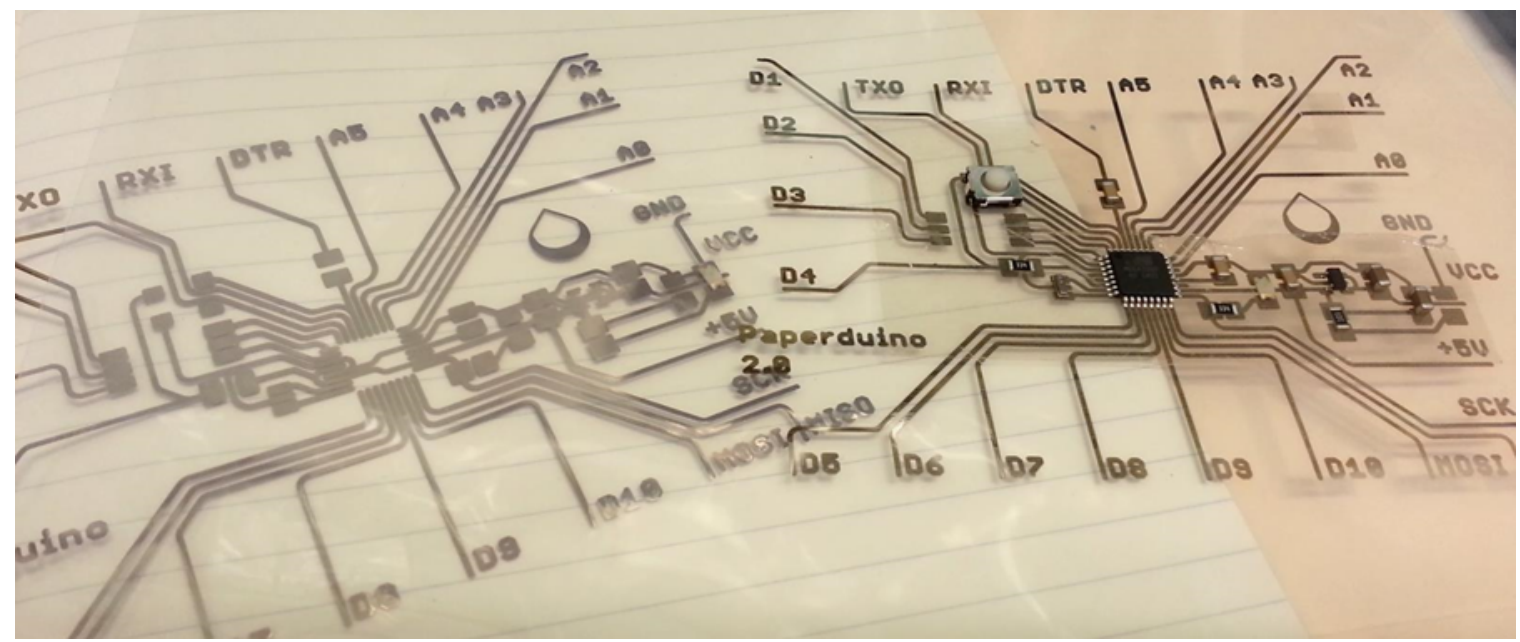

Figure 2.17. Examples of embedded complex circuit geometries in printed structures.

\subsection{INKJET PRINTED METAL ELECTRODES}

The combination of inkjet printing and low thermal budget photonic curing technology was explored for high performance metal contact development. The photonic curing system, as shown in Figure 2.18 (a), is capable of delivering a high intensity, short duration $(<20 \mathrm{~ms})$ broadband light pulse (200$1400 \mathrm{~nm}$ ) from a directed xenon flash lamp, to generate the very high processing temperatures required for thin film densification, recrystallization and annealing, but without damaging the underlying temperature-sensitive substrates or co-integrated electronics. The photonic curing system is capable of delivering maximum peak power in excess of $20 \mathrm{~kW} / \mathrm{cm}^{2}$ while the pulse width can be maintained as short as 30 microseconds.

\section{Unique Pulse Thermal Processing}

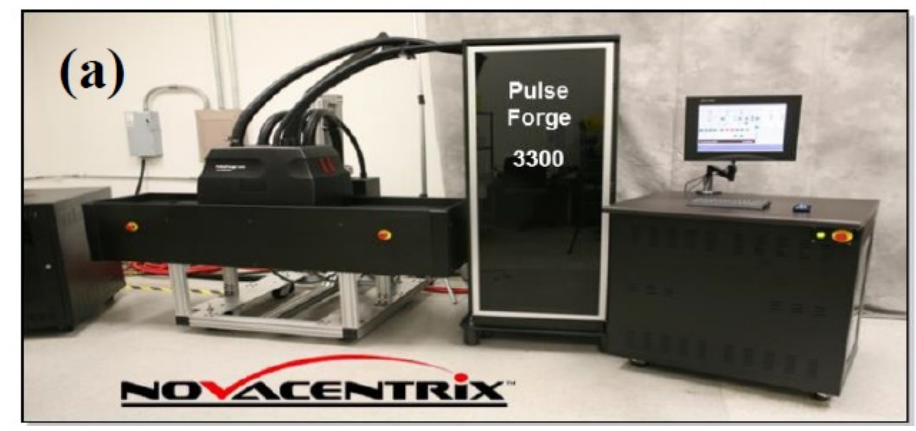

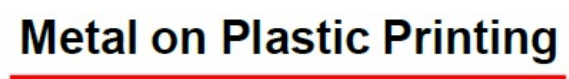

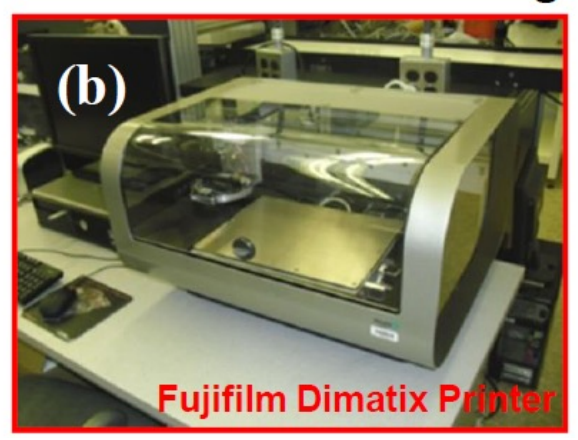

Figure 2.18. (a) PulseForge 3300 Photonic curing system and (b) DMP-2831 materials printer used for the development of silver electrode patterns on flexible plastic substrates.

The inkjet printer, shown in Figure 2.18(b), allows the deposition of fluidic materials on flexible substrates, utilizing a disposable piezo inkjet cartridge. The printer can create and define patterns over an area of about $200 \times 300 \mathrm{~mm}$ and handle substrates up to $25 \mathrm{~mm}$ thick with an adjustable $\mathrm{Z}$ height. The temperature of the vacuum platen, which secures the substrate in place, can be adjusted up to $60^{\circ} \mathrm{C}$. A waveform editor and a drop-watch camera system allows manipulation of the electronic pulses to the piezo jetting device for optimization of the drop characteristics as it is ejected from the nozzle. This system enables easy printing of structures and samples for process verification and prototype creation. The most unique feature of this table top printing system is the printhead 
itself. To minimize waste of expensive fluids, each cartridge reservoir has a capacity of $1.5 \mathrm{ml}$. Cartridges can easily be replaced to facilitate printing of a series of fluids. Each single-use cartridge has 16 nozzles linearly spaced at 254 microns with typical drop sizes of 1 and 10 picoliters. In the present work, $10 \mathrm{pL}$ ink-cartridges were used for the printing of silver electrode patterns.

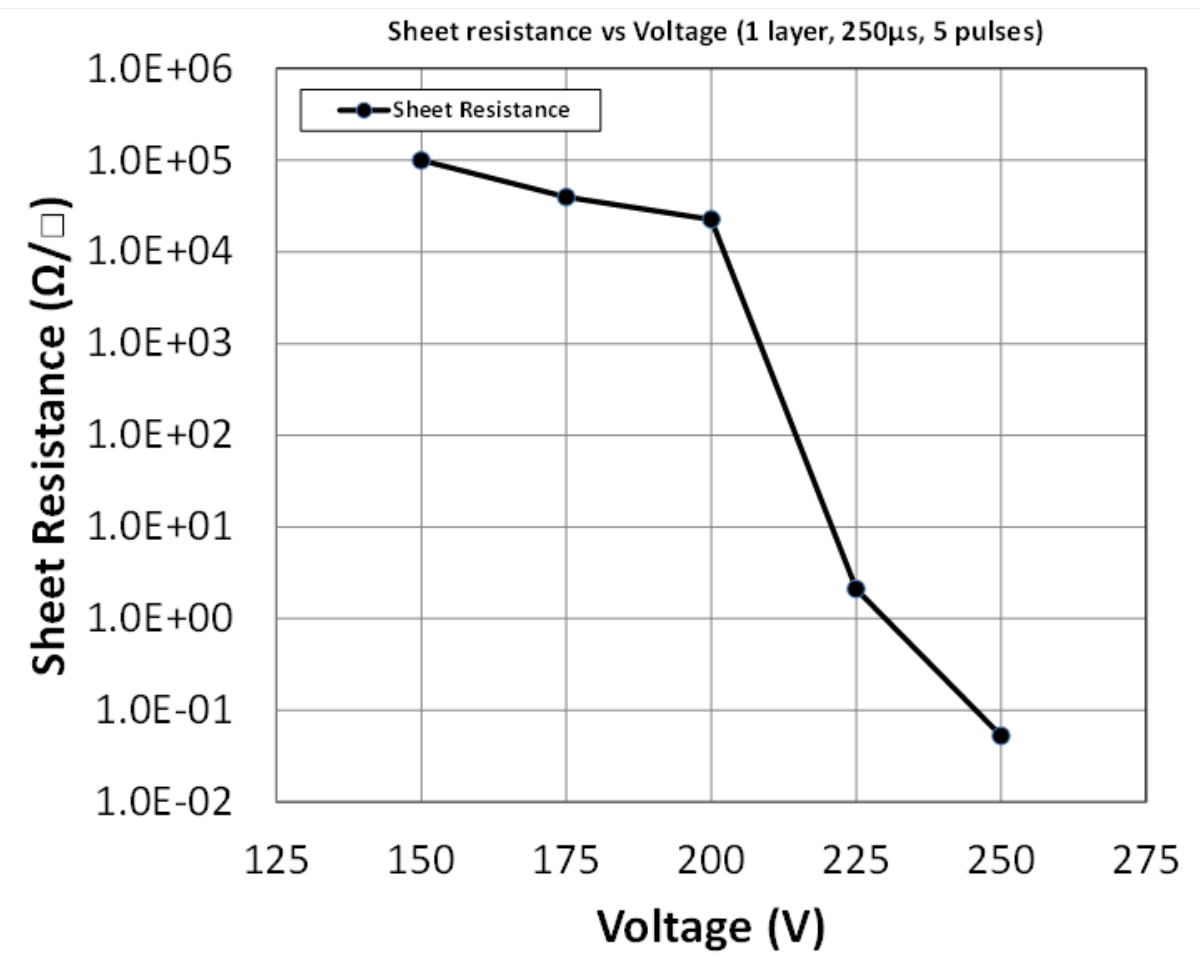

Figure 2.19. The impact of photonic curing on the electrical conductivity of printed silver electrodes.

The impact of photonic curing on the sheet resistance of a printed silver resistor is shown in Figure 2.19. The Ag patterns were printed on PET-based Novele substrates, which are suitable for low-cost and low-temperature applications. The photonic curing space was defined by the thermal-damage threshold of the flexible substrates. The impact of the $250 \mu$ s wide photonic pulses on the sheet resistance of the Ag line was analyzed, and the results shown in Figure 2.20, over the applied voltage range of 150-250V. The sheet resistance remained high over the voltage range of 150-200V, while an increase in the applied voltage to $225 \mathrm{~V}$ resulted in a significant reduction in sheet resistance to a value of $2.1 \Omega / S q$, which is suitable for interconnect applications. As the applied voltage was further increased to $250 \mathrm{~V}$, the sheet resistance value decreased by an order of magnitude to about $5.3 \times 10^{-2}$ $\Omega / S q$. 


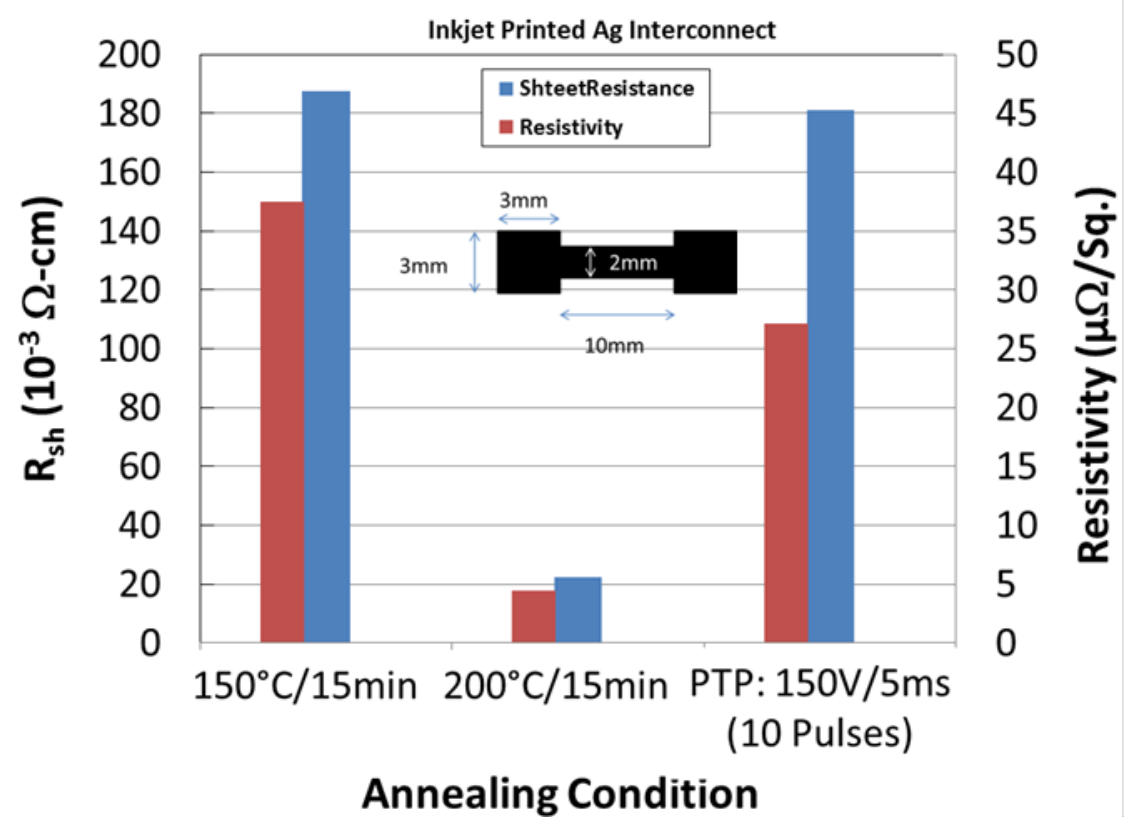

Figure 2.20. Impacts of photonic curing and conventional hot-plate sintering methods on the electrical properties of inkjet printed Ag electrode patterns.

The efficiency of the photonic curing process was evaluated by establishing a comparison with the convention hot-plate sintering method. Figure 2.20 shows the effects of photonic curing and hot-plate sintering on the electrical properties of a printed silver resistor. It was possible to achieve a sheet resistance value below $100 \mathrm{~m} \Omega / \mathrm{Sq}$ by photonic curing at a pulse-width of $5 \mathrm{~ms}$. The electrical properties were comparable to those achieved by conventional sintering at $150^{\circ} \mathrm{C}$, and the observed electrical performance of printed silver patterns is suitable for flexible electronic applications. The photonic curing process was optimized for further reductions in the silver metal-line resistance, while the multi-layer printing option was also available to further improve the electrical performance of the interconnects in all-printed electronic applications.

The inkjet printing technique offers simple and inexpensive processing of interdigitated electrodes (IDEs) for advanced sensor and electronic-circuit integration on flexible substrates. The IDE geometry can be tailored to achieve the desired signal response from the integrated sensor or other electronic components and devices. The digital printing method, using a CAD file, eliminates the costly mask redesign step to accommodate the modified designs. Additionally, the printing process eliminates the deposition, photolithography, and etching steps that are typically required in a cleanroom environment to define similar electrode configurations.

Figure 2.21 shows well-defined IDE patterns printed on PET substrates. The optimum line-definition was achieved using a drop-spacing of $20 \mu \mathrm{m}$. The electrical waveform and nozzle voltages were adjusted to achieve a consistent drop velocity of about $7.5 \mathrm{~m} / \mathrm{s}$. The platen temperature was maintained at $50^{\circ} \mathrm{C}$ during printing. The print quality and electrical performance of IDE patterns were evaluated in terms of the inter-electrode capacitance. A capacitance value of $17.5 \mathrm{pF}$ was measured for the integrated capacitor design on low dielectric constant PET substrates indicating well-controlled finger spacing and metal line-width. The observed results show promise for the development of flexible electronic circuits employing inkjet printed interconnects, electrode patterns, and passive electronic components. 


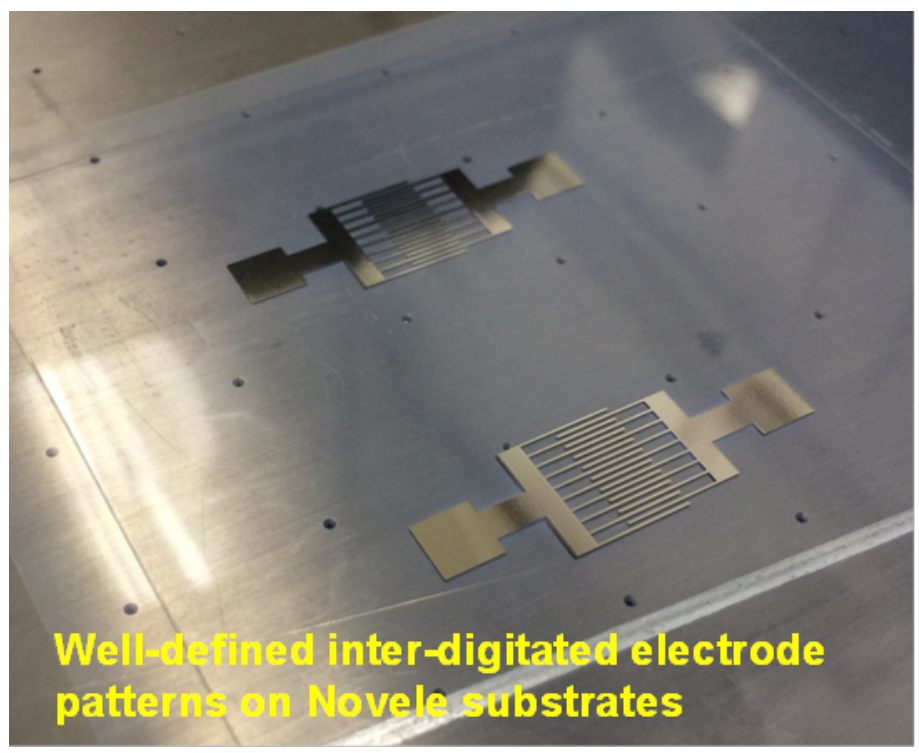

Figure 2.21. Inkjet printed interdigitated electrode patterns on flexible PET substrates.

\subsection{INKJET PRINTED METAL PATTERNS}

The low thermal budget of the photonic curing technology was explored for high performance metal contact development. Sheet resistance values below $100 \mathrm{~m} \Omega / S q$ were successfully achieved on flexible substrates using photonic curing. The electrical properties are comparable to those achieved by conventional sintering at $150^{\circ} \mathrm{C}$. The observed electrical performance of printed silver patterns is suitable for flexible electronic applications. However, the flexibility and mechanical reliability of printed metals are critical considerations for advanced device development and manufacturing technology integration.
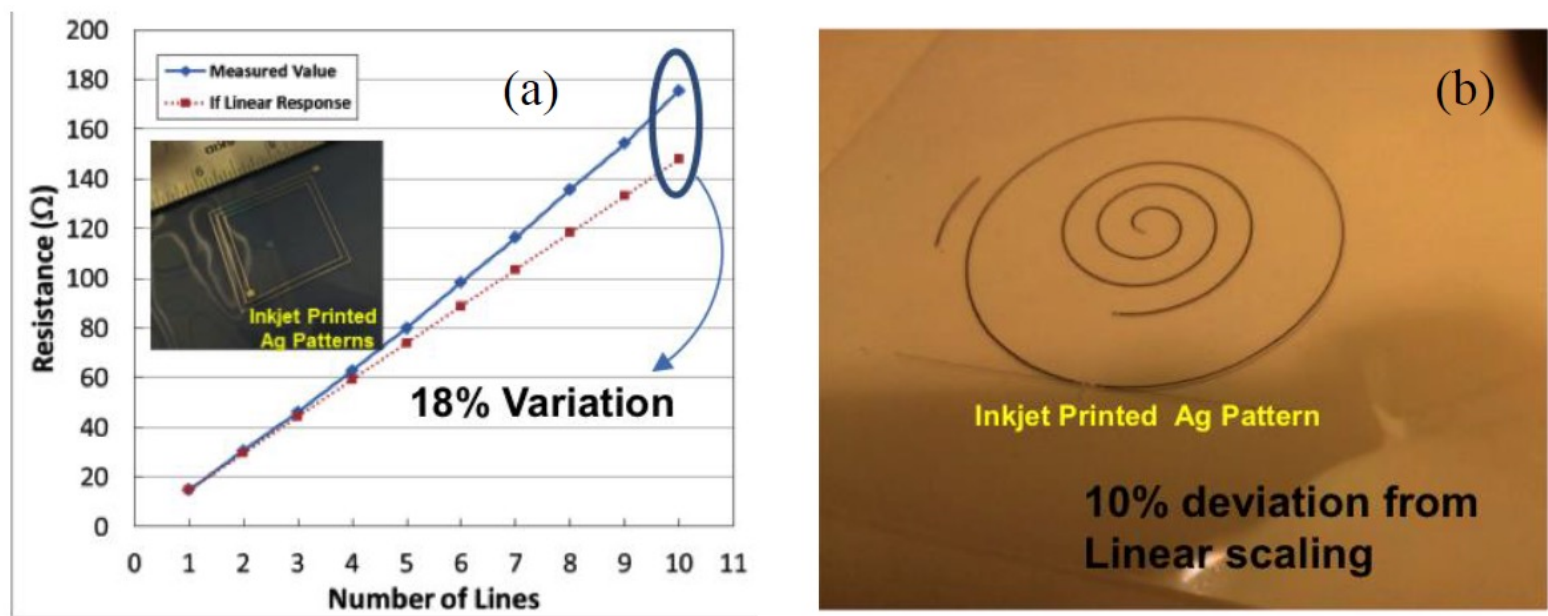

Figure 2.22. Printed Ag test structures to evaluate resistance scaling: (a) Rectangular and (b) Spiral.

Figure 2.22 shows an attempt made to analyze the impact of line definition on the scalability of line resistance. For $90^{\circ}$ transitions in the printed pattern, a deviation of $18 \%$ from the expected linear response was measured for a test pattern with ten turns as shown in Figure 2.22(a). A spiral of the same length as the rectangular pattern with ten $90^{\circ}$ turns was also printed, as shown in Figure 2.22(b), 
to analyze the impact of line definition. The spiral pattern showed a significant improvement in the resistance scaling indicating that curved transitions lead to a better line definition. The observed results suggest that the spiral pattern design should also be considered when printing metal interconnects and electrode patterns.

\subsection{PRINTED POLYMER SUBSTRATES FOR STRAIN GAUGES}

Six polymer substrates were prepared by additive printing techniques, to conduct mechanical testing (Figure 2.23). These structures were printed using the Stratasys Objet24 3D printer using the polymer Vero White Plus FullCure 835 UV polymer.

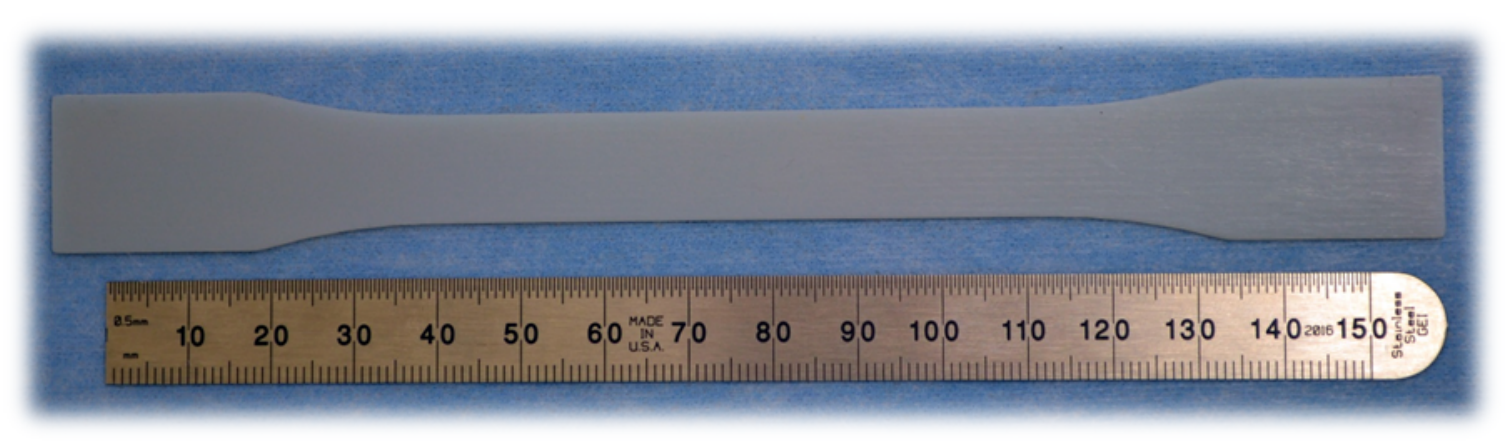

Figure 2.23. Additive printed polymer substrates for strain testing.

The substrates were polished with several grit polish levels to obtain better secondary print surface before proceeding to encapsulate the gauge patterns (Figure 2.24). However, we found that this tends to promote print instabilities, especially for coarser final polishing.

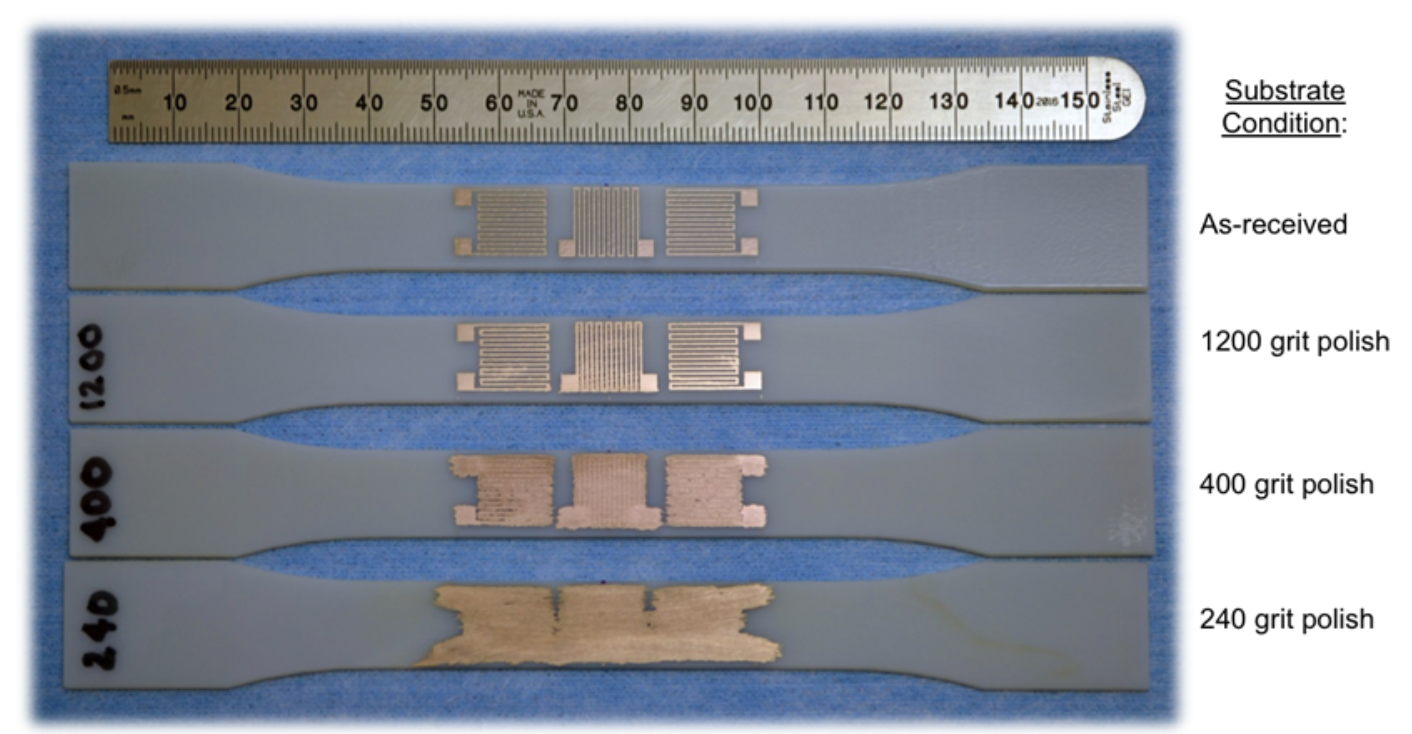

Figure 2.24. Polishing of the printed structures with several grit polish levels.

The printed lines were continuous but appeared to exhibit wrinkles, which might affect the performance of the strain gauge. A close up of the wrinkled structures is shown in Figure 2.25(a) and 2.25(b). Further analysis revealed that the wrinkles are folds several micrometer in height (Figure $2.25(\mathrm{c})$ ), and wrinkles of this size and density could potentially distort the behavior of a gauge where unfolding rather than straining occurs upon elongation. 

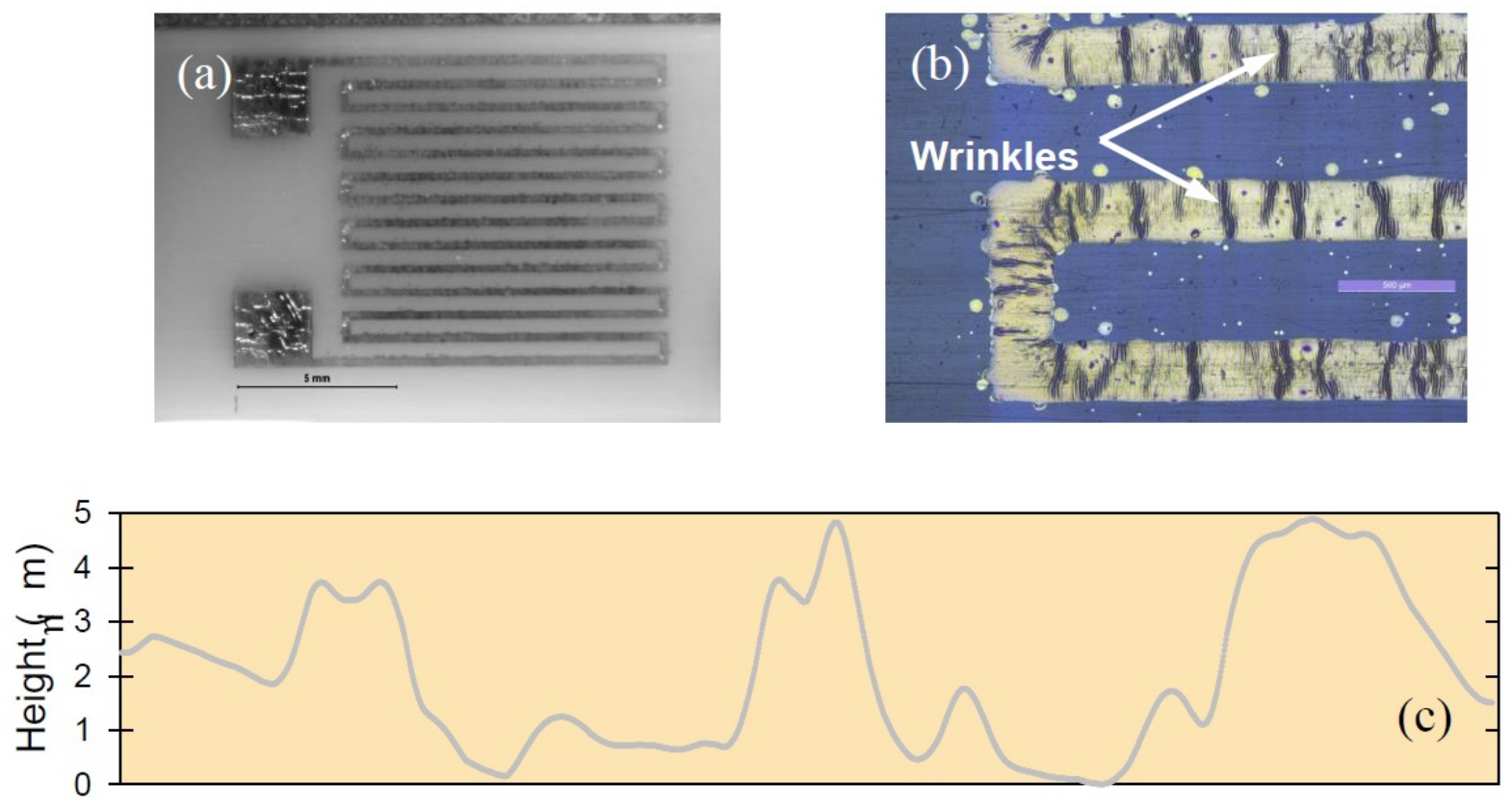

Figure 2.25. A close up of the wrinkled structure of the polishing of printed structures. 


\section{TASK 3: LOW COST PRINTED SENSORS}

The objective of this task was to demonstrate low cost printed sensor development by non-vacuum deposition techniques employing low thermal budget photonic curing. This was to involve cointegration of metal and/or semiconductor layers to establish a diverse sensor technology platform. The initial application for the low cost sensor technology was to be a thin film temperature sensor for energy efficient buildings, defense applications, and manufacturing diagnostics. The sensor was to be passive thin film piezoelectric design, and was to communicate with local receivers using radio frequency surface acoustic wave technology (RF-SAW), with the goal of sensing temperature changes based on physical changes in the conductive lines. The research performed in this project was to characterize in-house fabrication methodologies available for direct-write sensors and radio frequency $(\mathrm{RF})$ antennas that can be combined to produce a fully passive sensor that is readily reproducible by ink jet, screen, aerosol jet or other direct-write techniques.

\subsection{COMPLETE SAW DEVICE STRUCTURE}

A schematic of the complete device structure with typical dimensions of various components for a high frequency design is shown in Figure 3.1. A simple SAW device was designed and fabricated as a first step. A delay line structure was fabricated to demonstrate SAW operation and a simple transducer with reflector structure to demonstrate sensor operation. The first design template contained an array of RF-SAW structures of various feature sizes ranging from 9.8 to 500 microns. The change in reflector time delay due to external stimuli, such as temperature change, is tracked. Having two tracks allows one track to be used for temperature compensation while the other track may be used to sense another parameter. By fabricating this array of features, we were able to characterize the line width, line spacing (pitch) and coating thickness, and achieve reproducibly. Once the fabrication limitations were understood, various substrate materials and inks were explored to optimize device performance and minimize feature sizes with a line width goal of 0.8 microns or smaller.

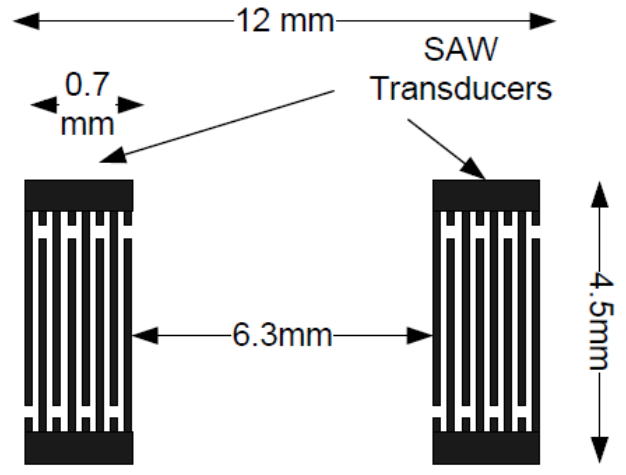

(a)

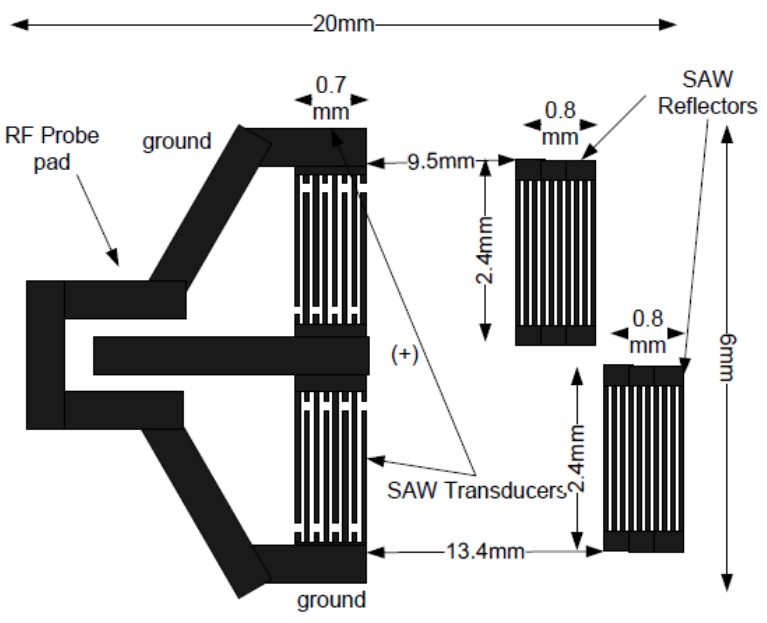

(b)

Figure 3.1. (a) SAW Delay line structure with two transducers (the electrode widths $=19.8$ um for 40 MHz operation on LiNbO3), (b) A simple single frequency sensor with two tracks, each having a reflector which will produce a reflection at a different time delay. 


\subsubsection{Thin Film Development}

A simple delay line structure can be realized on suitable materials such as $\mathrm{YZ}-\mathrm{LiNbO}_{3}, \mathrm{PZT}$, and $\mathrm{ZnO}$. The SAW response depends on the substrate and metal electrode quality. The SAW coupling, velocity and reflectivity of the electrodes are the parameters that were considered for the sensor design. The focus was on the development of low temperature $\mathrm{ZnO}$ thin films as the first step. The films were deposited by a sputtering technique on Si and plastic substrates. The thin film properties and SAW device characteristics were correlated with modeling to establish the impact of dispersion characteristics for high performance device development. The metal thin film development focused on $\mathrm{Ag}$ and $\mathrm{Al}$ thin films, which were processed by e-beam evaporation and inkjet printing techniques.

\subsubsection{Metal Electrodes}

The e-beam evaporation process was optimized for low temperature deposition of metal thin films on flexible substrates. We targeted a process temperature of less than $150^{\circ} \mathrm{C}$ for the various metal films which was suitable for plastic substrates of interest. Figure 3.2 shows the Ag thin film patterns deposited on various plastic substrates under room temperature conditions. A sheet resistance of $0.096 \mathrm{Ohms} / \mathrm{Sq}$. was measured on 300-nm-thick Ag films, which was suitable for diverse interconnect and electrode designs. The measured resistivity of the films was about $2.88 \times 10^{-6} \mathrm{Ohm}-$ $\mathrm{cm}$ indicating that the room-temperature metal deposition process was suitable for plastic integrated device development.

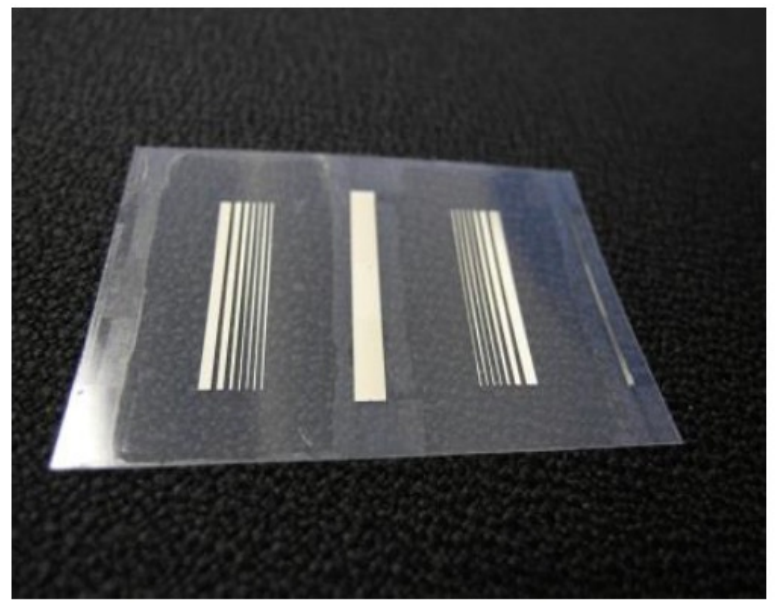

(a)

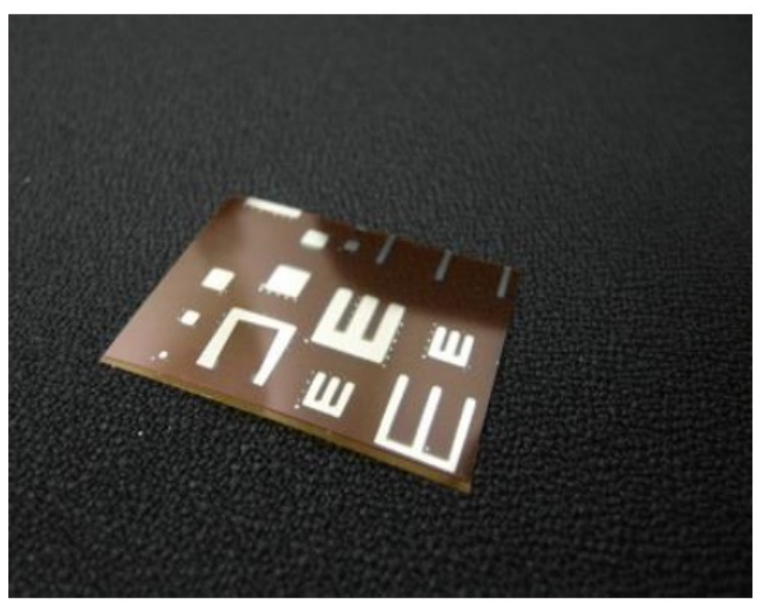

(b)

Figure 3.2. Room temperature Ag thin film patterns deposited on plastic substrates by e-beam evaporation technique: (a) Novelle ${ }^{\circledR}$ PET, (b) PET.

\subsection{SENSOR DESIGN}

The efforts in this phase of the Project involved the design, development, fabrication and performance validation/testing of passive wireless sensors that may be mass-produced using techniques developed within the Project. Such sensor development activities revolved around radio frequency surface acoustic wave (RF-SAW) techniques, which hold promise for fabrication using direct-write technologies. 
The ORNL RF-SAW efforts focused on modifying and scaling RF-SAW designs that were provided by Prof. Donald Malocha (University of Central Florida) and his post-doctoral assistants.

Investigations centered on a reference design set for operation in the $915 \mathrm{MHz}$ (ISM band) frequency range and then scaling the design to the $250 \mathrm{MHz}$ and $49 \mathrm{MHz}$ frequency range. The implications for direct write sensor printing were driven by the fact that the RF-SAW's operating frequency is inversely proportional to the line widths of the interdigitated "fingers" of the SAW device (Figure 3.3). While ORNL has the capability of producing a wide range of line widths, thereby providing the RF-SAWs with a wide range of operating frequencies, the project efforts were concentrated on using the 50 micrometer conductive ink printing capability - meaning that the RF-SAW designs operated at a lower frequency range than what they were designed for.

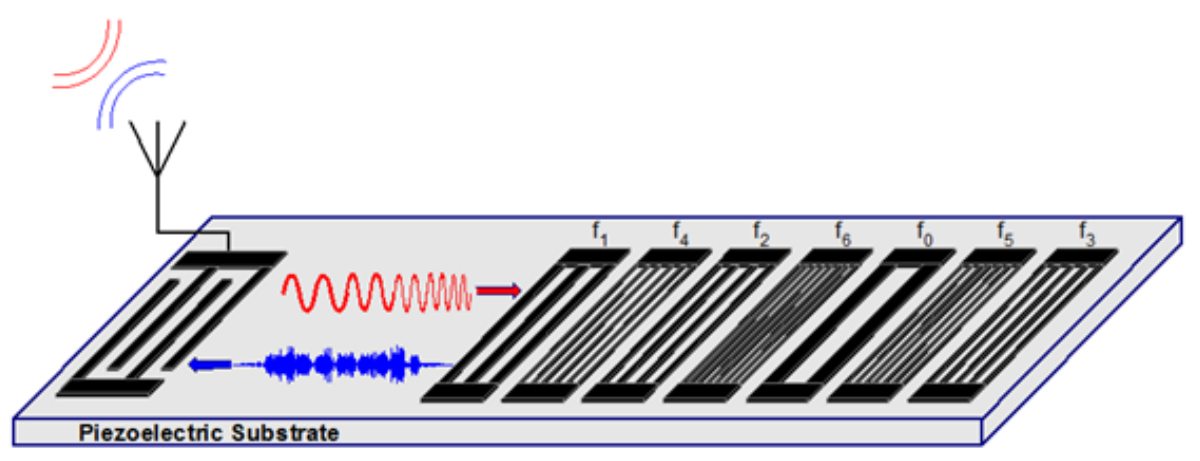

Figure 3.3. Representative 7-sensor element RF-SAW structure.

The broadband RF test and measurement equipment necessary to verify performance of the devices and components were assembled at ORNL, and a set of RF-SAW "reference" devices, designed to operate in the $915 \mathrm{MHz}$ frequency range, were designed and fabricated. An annotated photomicrograph of one of the devices is shown in Figure 3.4. The "coded reflectors" are using an orthogonal frequency coding technique, which allows for easy signal discrimination in the interrogated signal from which the sensor reading is extracted.

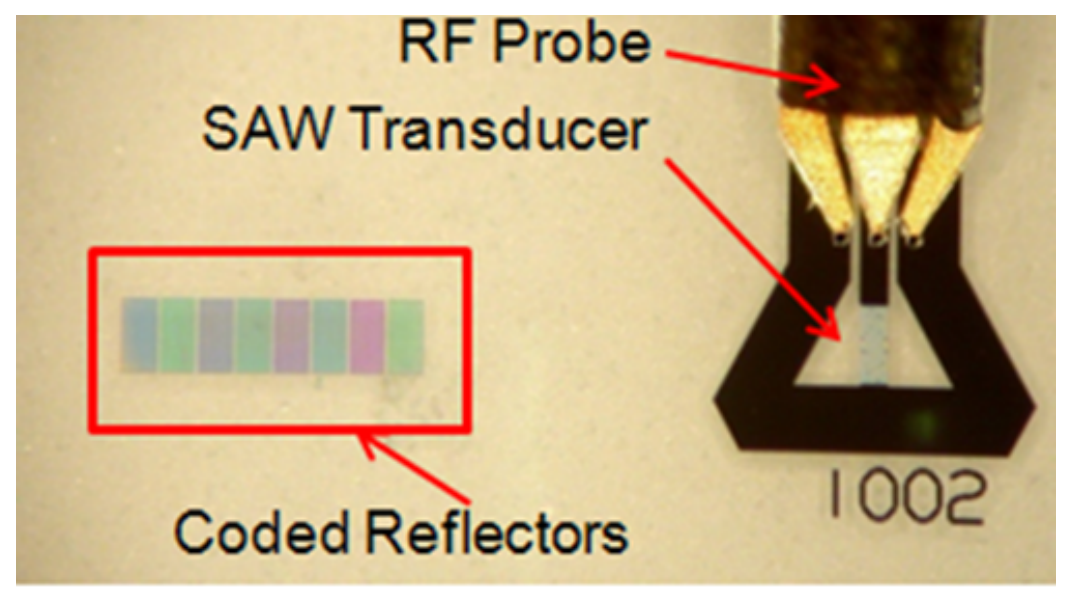

Figure 3.4. Photomicrograph of "reference" RF-SAW device.

Operation of the RF-SAW with sensors, either on-board (Figure 3.5) or off-board (Figure 3.6) relies on an interrogating RF signal (narrowband, pulsed, centered on the designed operating frequency) being received by the RF-SAW. The RF-SAW then generates a return signal that the test and measurement system receives and decodes. 
"Interrogation" of the $915 \mathrm{MHz}$ RF-SAW returned a frequency spectrum that was broad. FFT transformation of this spectrum revealed the temporal nature of the signal, from which the specific responses from the coded reflectors were observable, as shown in Figure 3.7.

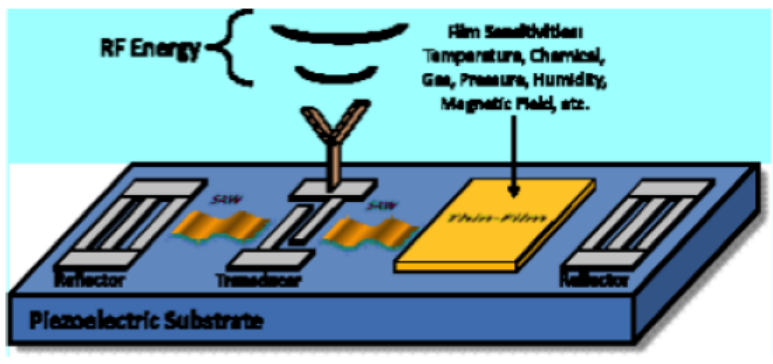

Figure 3.5. Design with on-board sensors.

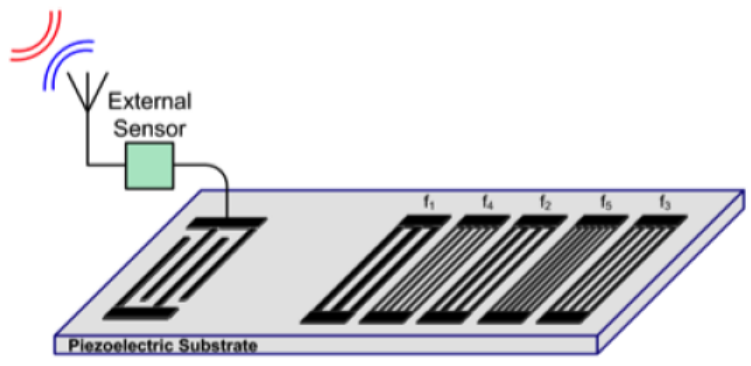

Figure 3.6. Design with off-board sensor.
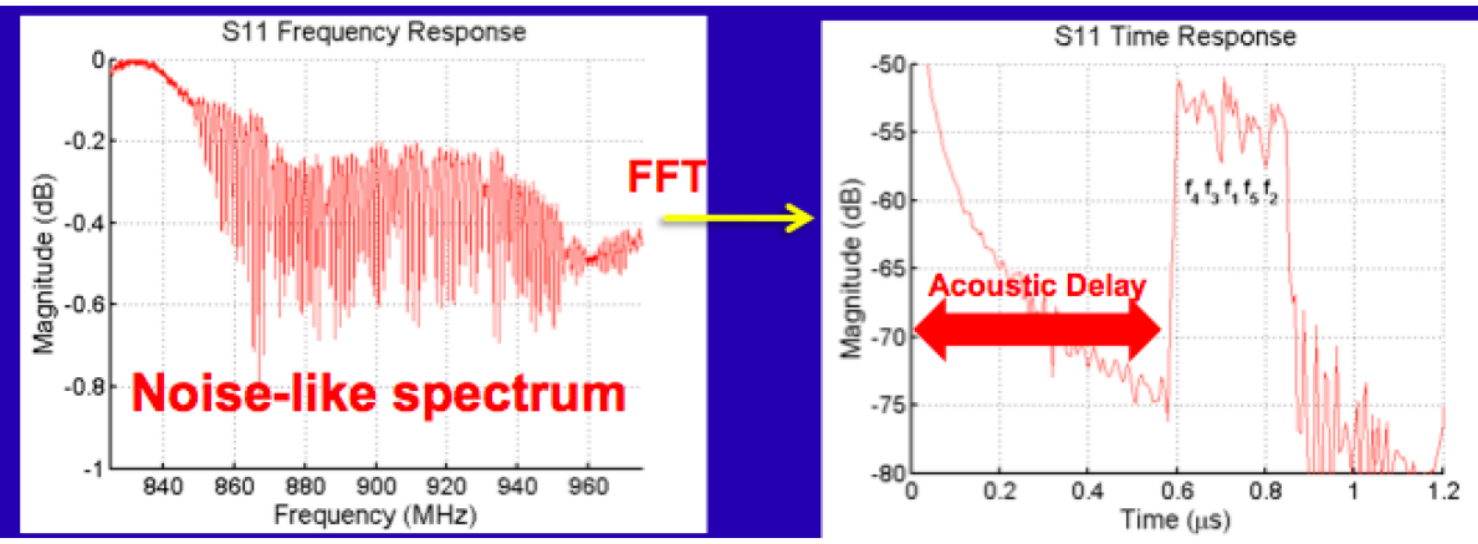

Figure 3.7. Broadband frequency and temporal response of the tested RF-SAW.

Deciphering the sensor signals occurs in either the frequency- or temporal-domain. Simulations were generated for the predicted response. A representative simulation and measured RF-SAW set of signals is shown in Figure 3.8.

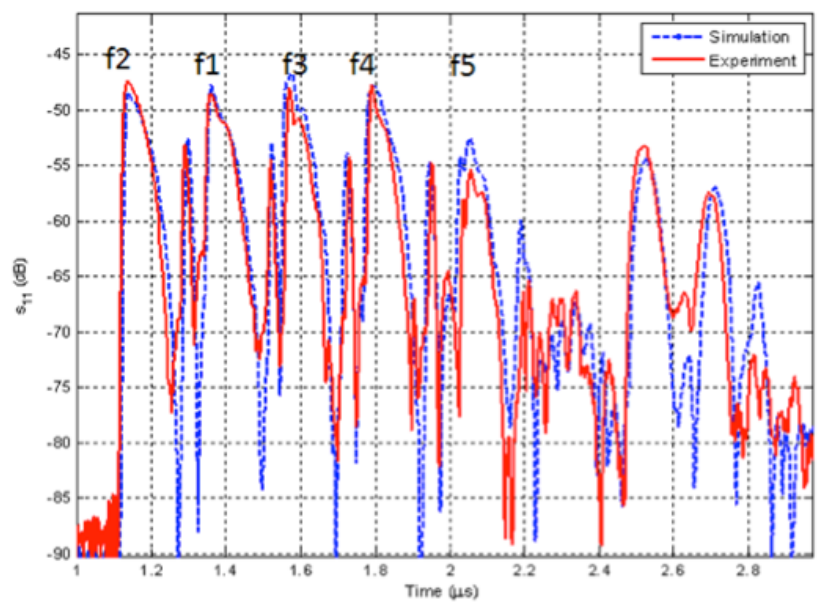

Figure 3.8. RF-SAW "readings" for configuration with 5 elements. 
In collaboration with Prof. Malocha, we investigated the use of Langatate (LGT) as a substrate for high temperature RF-SAW operation (sensing and communications). Preliminary results revealed that a design capable of sustained operation at $1400^{\circ} \mathrm{C}$ is possible. A photograph of a fabricated device, with a photomicrograph insert of the actual SAW structure, is shown in Figure 3.9. This device was tuned for $915 \mathrm{MHz}$ operation, but can be scaled to the 50 micrometer line width of the aerosol jet printers used in the project. A photograph of an RF-SAW on LGT after it has been subjected to $1400^{\circ} \mathrm{C}$ for 2 hours is shown in Figure 3.10.

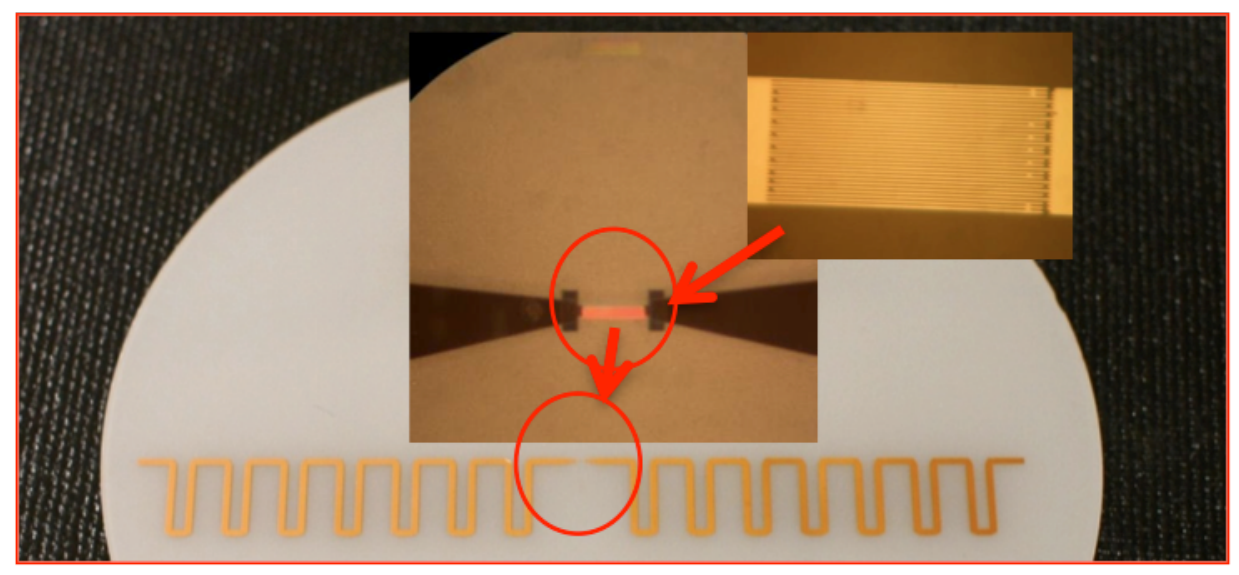

Figure 3.9. Annotated photomicrograph of an RF-SAW device capable of operation at high temperature.

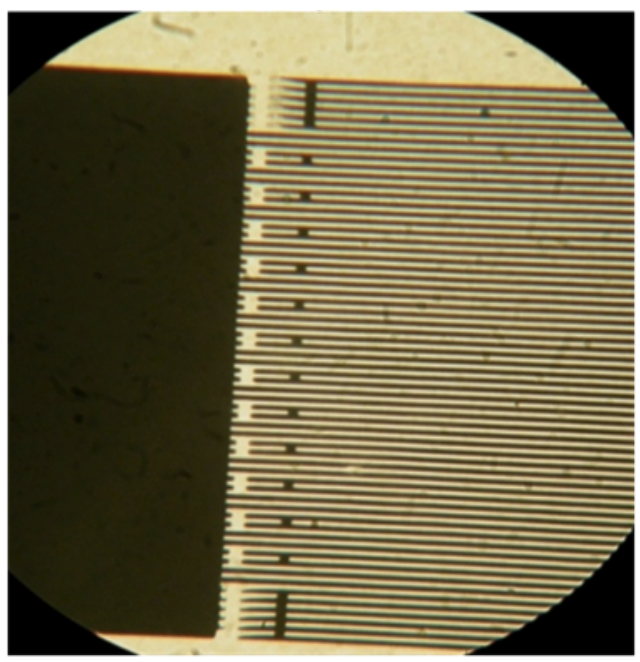

Figure 3.10. RF-SAW on LGT after 2 hours at $1400^{\circ} \mathrm{C}$.

The key findings from this study are as follows:

- The film thickness is best if it is approximately $1-2 \%$ of the operating wavelength. There is significant degradation in performance if the film thickness exceeds $4 \%$ of the operating wavelength.

- When using aluminum electrodes, the denser the metal, the thinner the metal thickness for optimal performance. For example, in the device shown in Figure 3.9 with Al electrodes, the optimal line thickness is in the $800 \mathrm{~nm}$ to $1500 \mathrm{~nm}$ range, but should absolutely not be more than 3 um thick.

- Examining layouts with differing positions for RF probe test points led to a reference dipole 
antenna structure suitable for testing (and calibrating) the RF test and measurement system. This dipole layout had a minimum layout line size of $9.8 \mathrm{um}$, and fits within a 5 " x 5" window. The reference dipole was not suitable for the 50 micrometer line width fabrication. Scaling of the system to this line width, with the accompanying decrease in RF operating frequency, is underway.

- Optimization of the RF-SAW in terms of overall performance was multivariate, with the most important parameters being antenna gain, fractional antenna bandwidth, and operating frequency. A representative tradeoff diagram is presented as Figure 3.11.

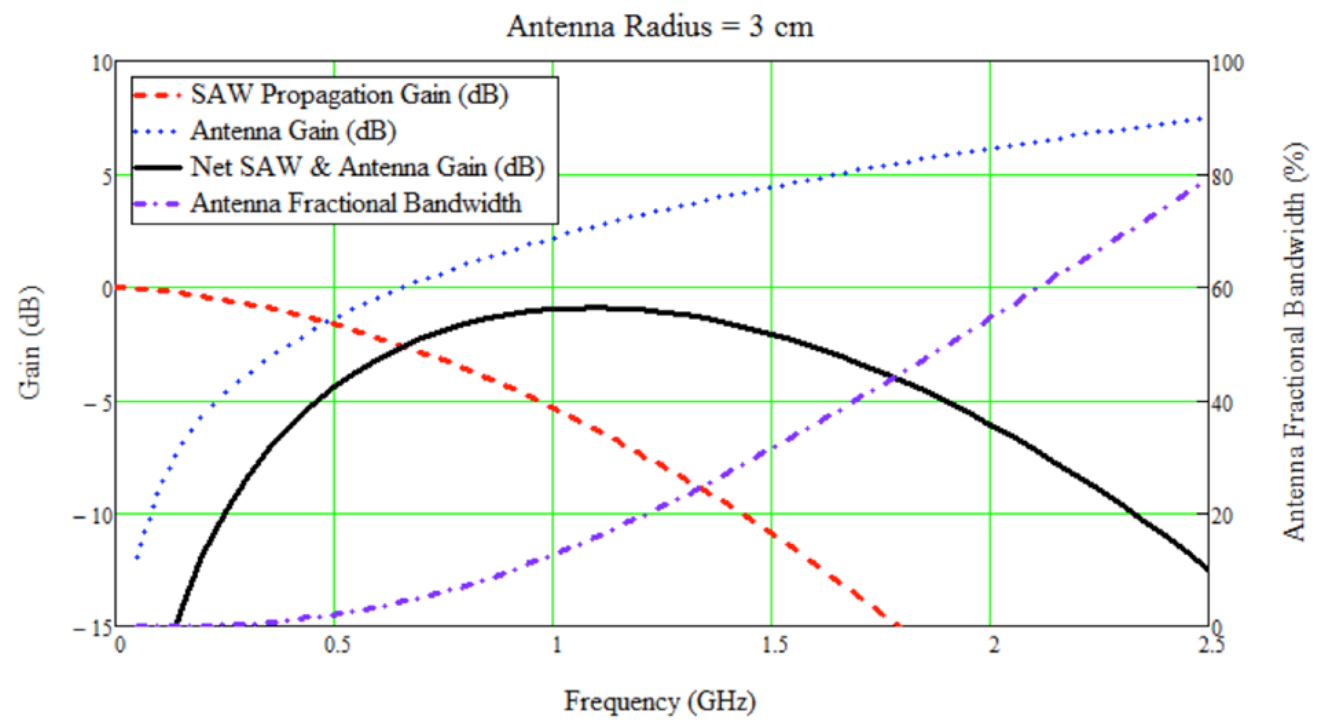

Figure 3.11. Optimization of an RF-SAW.

Given the line width limitations in the direct write devices, we have turned attention to harmonic operation. While this is suboptimal for realistic operation (antenna gains, etc.) it allows wider line width device fabrication while operating in higher frequency ISM bands.

\subsection{LOW TEMPERATURE ZnO THIN FILMS}

The low temperature semiconductor thin film development was focused on sputtered $\mathrm{ZnO}$ thin films. The pulsed-dc sputtering process was optimized to process high quality thin films at low temperatures suitable for integration on flexible plastic substrates. The sputtering process conditions for the investigation were as follows: $\mathrm{RF}$ Power $=400 \mathrm{~W}$, Pressure $=5 \mathrm{mTorr}$, Process Temperature $=25-200^{\circ} \mathrm{C}$, $\mathrm{Ar} / \mathrm{O}_{2}$ flow ratio $=0.25$, Pulsing Frequency $=300 \mathrm{kHz}$, Duty-cycle $=0.76$.

Figure 3.12 shows the properties of 78 -nm-thick films deposited at a temperature of $200^{\circ} \mathrm{C}$. The XRD results indicate the formation of predominantly c-axis oriented $\mathrm{ZnO}$ films, which are desired for high performance device development. A refractive index value of 2.066 was measured at $600 \mathrm{~nm}$ by ellipsometry technique, indicating the formation of a dense $\mathrm{ZnO}$ microstructure at a low temperature of $200^{\circ} \mathrm{C}$. 


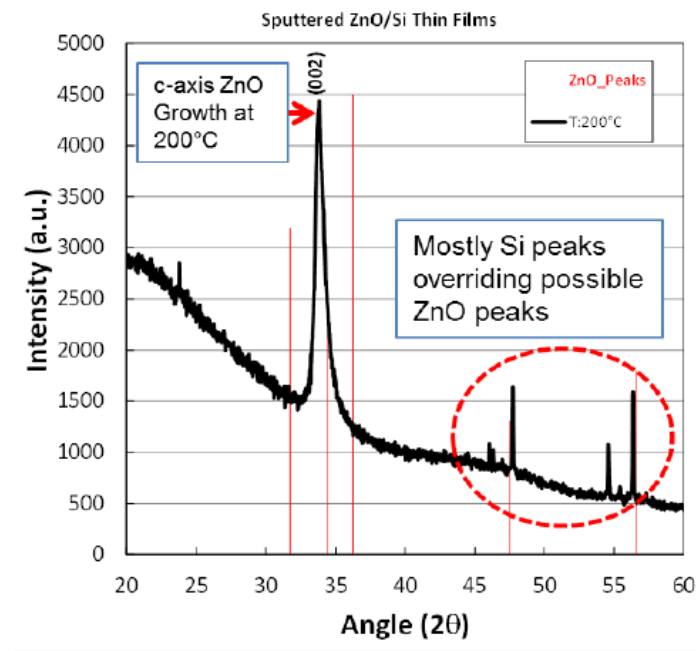

(a)

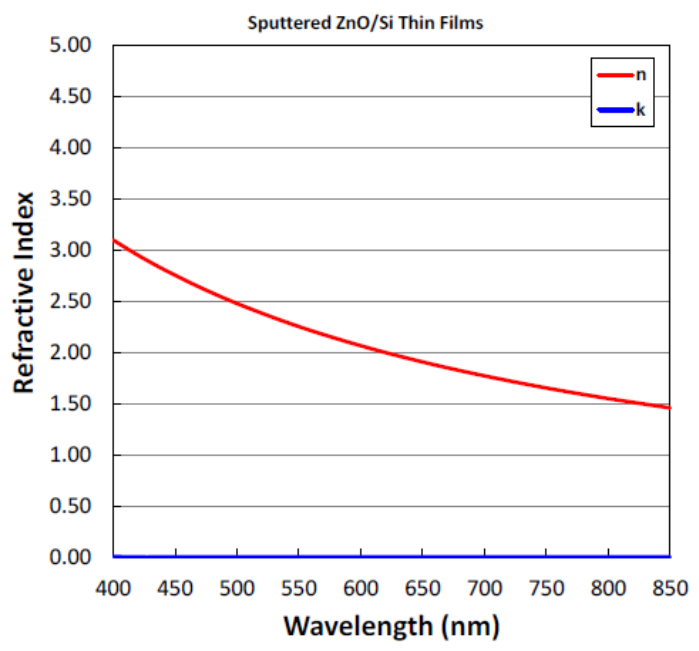

(b)

Figure 3.12. Properties of sputtered $\mathrm{ZnO}$ thin film processed at $200^{\circ} \mathrm{C}$ : (a) crystallinity, (b) optical n-k dispersion.

Figure 3.13(a) shows that the $\mathrm{ZnO}$ deposition rate was well maintained at around $0.76 \AA / \mathrm{s}$ in the temperature range of $100-200^{\circ} \mathrm{C}$. It decreased to about $0.60 \AA / \mathrm{s}$ at a deposition temperature of $25^{\circ} \mathrm{C}$. As shown in Figure 3.13(b), the pulsed-dc sputtering technique was effective in inducing c-axis orientation in the $\mathrm{ZnO}$ thin films even at a low temperature of $200^{\circ} \mathrm{C}$. The c-axis orientation was well-maintained even at a deposition temperature of $25^{\circ} \mathrm{C}$ which is promising for device development on flexible substrates.

\begin{tabular}{|l|c|}
\hline $\begin{array}{c}\text { Temperature } \\
\left({ }^{\circ} \mathrm{C}\right)\end{array}$ & $\begin{array}{c}\text { Deposition Rate } \\
(\AA \mathbf{A} / \mathbf{s})\end{array}$ \\
\hline 25 & 0.60 \\
\hline 100 & 0.76 \\
\hline 200 & 0.76 \\
\hline
\end{tabular}

(a)

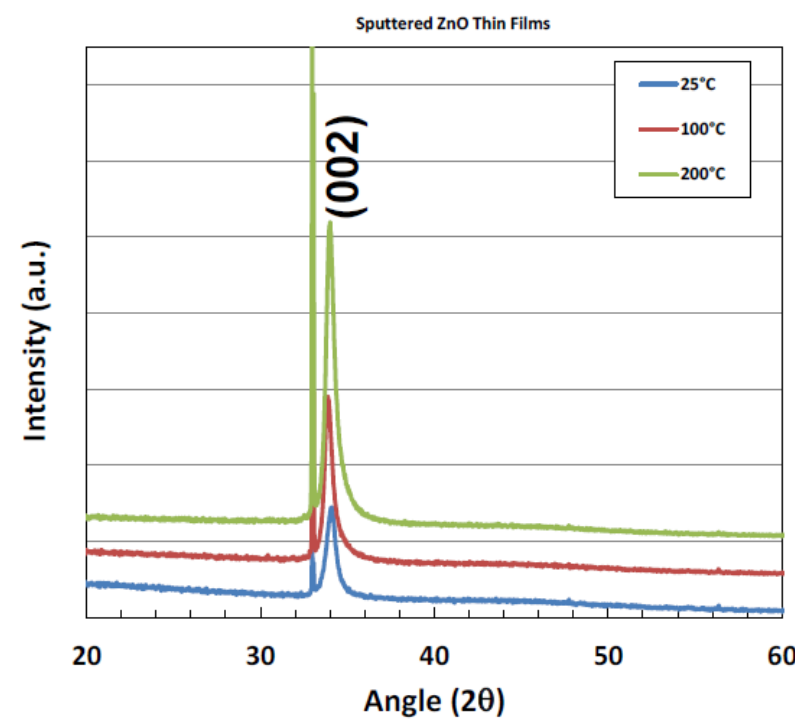

(b)

Figure 3.13. The effect of process temperature on the (a) deposition rate, and (b) crystallinity of $\mathrm{ZnO}$ thin films.

Figure 3.14(a) shows the optical transmittance of the films as a function of process temperature in the range of $25-200^{\circ} \mathrm{C}$. The $\mathrm{ZnO}$ thin films were deposited on fused quartz substrates for the optical 
transmission measurements. The optical transmittance of the film was greater than $80 \%$ in the wavelength range of $400-700 \mathrm{~nm}$ even at a deposition temperature of $25^{\circ} \mathrm{C}$. The optical quality of the films was analyzed by spectroscopic ellipsometry. Figure 3.14(b) shows the n-k dispersion characteristics of the $\mathrm{ZnO}$ thin films deposited on Si substrates. A decrease in process temperature to $25^{\circ} \mathrm{C}$ did not influence the $\mathrm{n}-\mathrm{k}$ dispersion characteristics appreciably indicating that the optical density of the films was well-maintained even down to a process temperature of $25^{\circ} \mathrm{C}$. Overall, the pulsed-dc sputtering technique shows promise for the low temperature processing of the semiconducting $\mathrm{ZnO}$ thin films at plastic compatible process temperatures. The observed results suggest that a combination of the low temperature $\mathrm{ZnO}$ thin films and low-thermal budget pulse thermal processing technique is worth exploring for highly functional device development on flexible substrates.

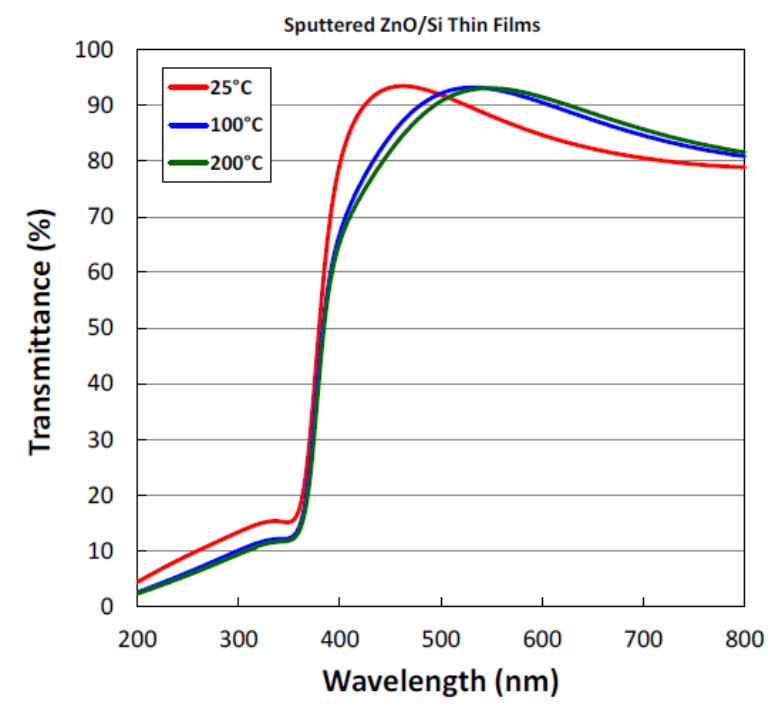

(a)

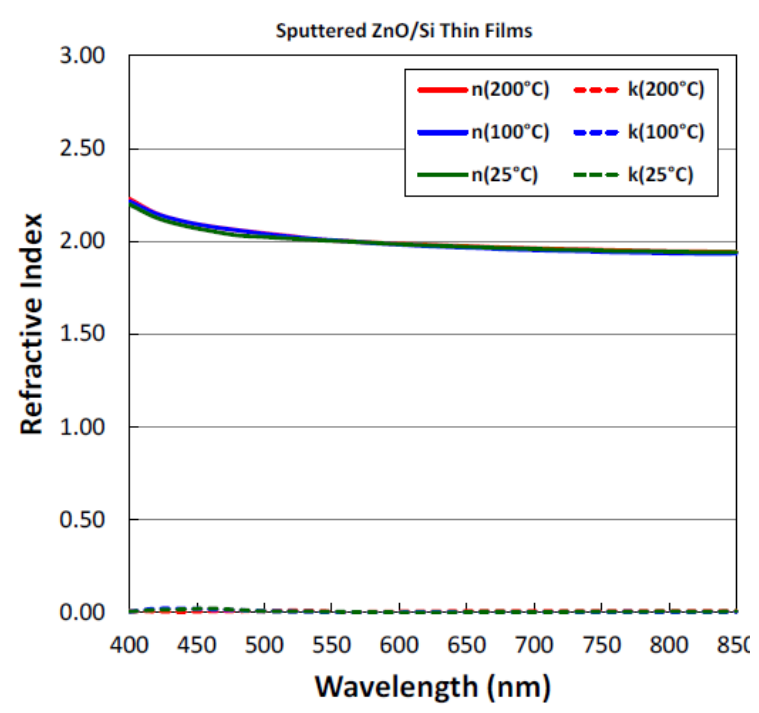

(b)

Figure 3.14. The impact of process temperature on the (a) optical transmittance, and (b) n-k dispersion characteristics.

Table 3.1. Various metal oxide thin film configurations under investigation for the flexible electronic applications

\begin{tabular}{|c|c|}
\hline Metal Oxide Film Configurations & Characterization \\
\hline $\begin{array}{ll}\cdot & I T O / P I \\
\cdot & \mathrm{ZnO} / \mathrm{PI} \\
\cdot & \mathrm{TTO} / \mathrm{ZnO} / \mathrm{PI} \\
\cdot & \mathrm{ITO} / \mathrm{ZnO} / \mathrm{ITO} / \mathrm{PI} \\
\cdot & (\mathrm{ITO} \text { or } \mathrm{ZnO}) / \mathrm{SiO}_{2} / \mathrm{Si}\end{array}$ & $\begin{array}{l}\text { - Microstructural } \\
\text { - Optical } \\
\text { - Electrical } \\
\text { - UV Response }\end{array}$ \\
\hline
\end{tabular}




\subsection{TRANSPARENT CONDUCTING OXIDE THIN FILMS}

The focus for the next stage of the project was on the development of high quality transparent conducting oxide (TCO) thin films on plastic substrates. The details of various metal oxide thin film configurations under investigation are listed in Table 3.1. The initial investigation focused on room temperature processed $\mathrm{ZnO}$ and ITO thin films. The pulse thermal processing (PTP) technique was used for the low temperature annealing of the TCO thin films for plastic integration. The results of our initial investigation of the PTP technique are also discussed in the following sections.

\subsubsection{Room Temperature ITO Deposition}

Indium Tin Oxide (ITO) is a transparent conducting oxide suitable for electrical interconnects in diverse electronic devices. We attempted to process the ITO thin films on plastic substrate for lowcost sensor development. The ITO thin films were also deposited by pulsed dc sputtering technique to process high quality films at process temperatures below $100^{\circ} \mathrm{C}$. Table 3.2 lists the typical sputtering conditions, and the corresponding ITO thin film properties. A low resistivity of the order of $1.26 \times 10^{-3}$ was achieved for films deposited on quartz substrates at room temperature without any post-deposition thermal treatment. The observed results show the effectiveness of the pulsed dc sputtering mode in depositing high quality thin films at low process temperatures $\left(<100^{\circ} \mathrm{C}\right)$.

Table 3.2. Room temperature deposition of ITO thin films by pulsed de sputtering technique

\begin{tabular}{|r|r|r|r|}
\hline Power $(\mathrm{W})$ & Pressure (mTorr) & Dep-Rate $(\AA / \mathrm{s})$ & Resistivity $(\Omega$-cm) \\
\hline 150 & 10 & 0.50 & $2.94 \mathrm{E}-03$ \\
\hline 150 & 5 & 0.63 & $1.26 \mathrm{E}-03$ \\
\hline 200 & 5 & 1.03 & $1.86 \mathrm{E}-03$ \\
\hline
\end{tabular}

The ITO thin films were also deposited on plastic substrates to analyze the impact of substrate on thin film quality. The films deposited on plastic substrates were also highly transparent as shown in Fig. 3.15(a). The ITO thin film transmittance was comparable on plastic and quartz substrates as shown in Fig. 3.15(b), indicating similar film quality. The intrinsic ITO thin film transmittance was greater than $75 \%$ in the visible part of the spectrum as confirmed by UV-VIS-IR spectrophotometer.

ITO/PI: RT Deposition

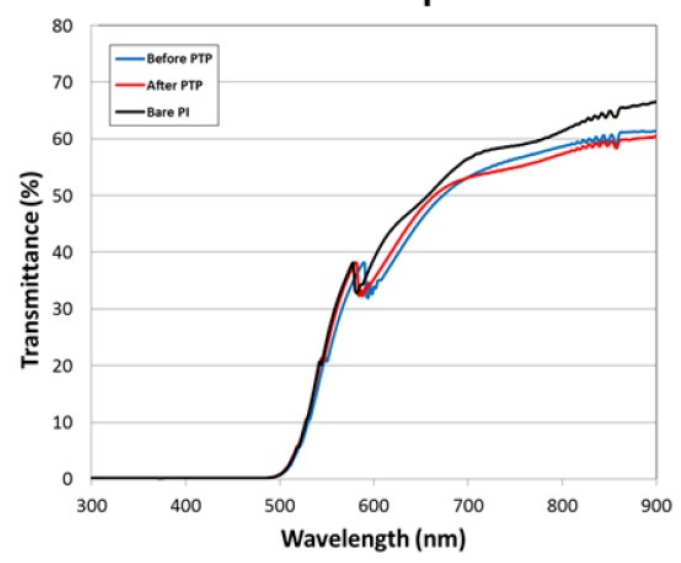

(a)
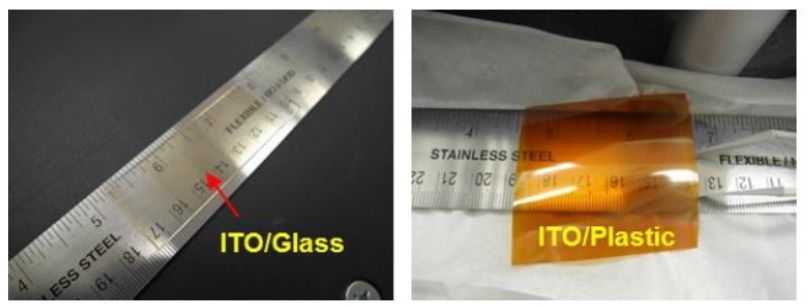

(b)

Figure 3.15. The optical transmittance of the room temperature deposited ITO thin films: (a) transmittance of ITP/PI thin films, (b) prototypes on quartz and plastic substrates. 
The impact of the low thermal budget pulse thermal processing (PTP) on the electrical conductivity of the ITO/Plastic thin films was also analyzed. The PTP processing was carried out in the PulseForge 3300 system using $220 \mathrm{~V} / 5 \mathrm{~ms}$ pulses. Figure 3.16 shows the impact of 10 pulses on the sheet resistance $\left(\mathrm{R}_{\mathrm{sh}}\right)$ of the room temperature deposited ITO films. The $\mathrm{R}_{\text {sh }}$ values decreased substantially from 129 to $50 \Omega / / \mathrm{Sq}$. after PTP processing which is promising for processing high quality metaloxide thin films on plastic substrates for advanced sensor development. Figure 3.16 also shows a resistivity below $10^{-3} \mathrm{Ohm}-\mathrm{cm}$.

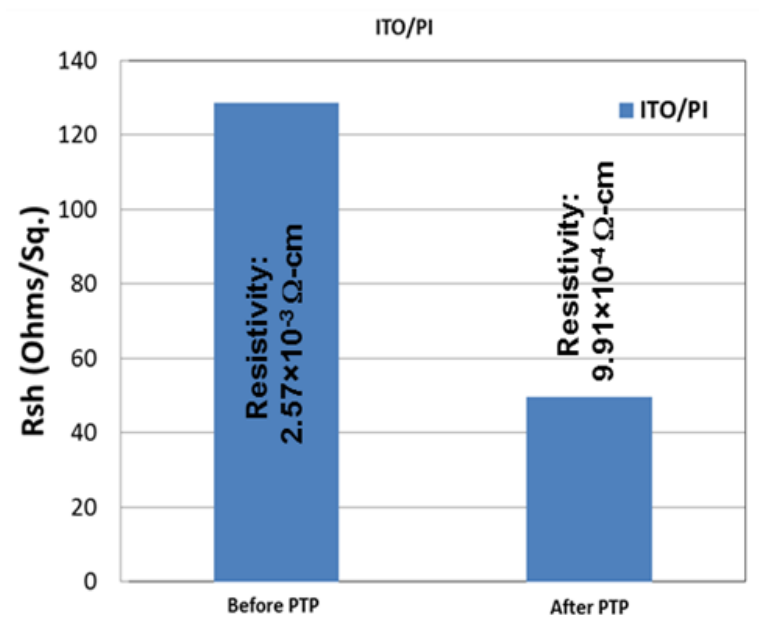

Figure 3.16. The impact of low thermal budget pulse thermal processing on the sheet resistance of ITO thin films sputtered at room temperature.

The impact of PTP processing on the electrical conductivity of room temperature deposited ITO thin films was further studied by depositing ITO films on $\mathrm{SiO}_{2} / \mathrm{Si}$ substrates by the pulsed-dc sputtering technique. The results are shown in Fig. 3.17. The as-deposited ITO films showed a low sheetresistance value of about $75 \Omega / \mathrm{Sq}$ showing the effectiveness of the pulsed dc sputtering mode in depositing high quality thin films at low process temperatures $\left(<100^{\circ} \mathrm{C}\right)$.

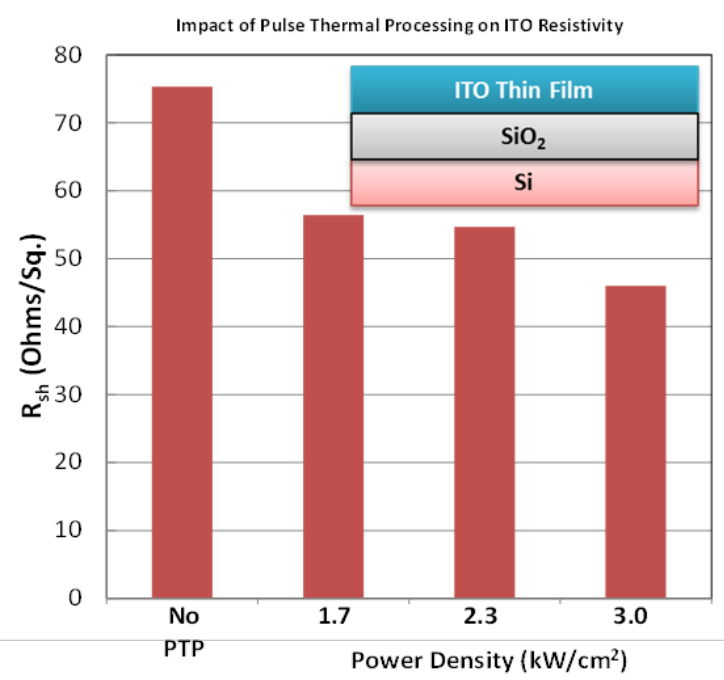

Figure 3.17. The impact of pulse thermal processing on the electrical performance of room temperature processed ITO thin films. 
The ITO thin films deposited on plastic substrates showed high optical transparency $(>75 \%$ in visible part of the spectrum - Figure 3.15). The PTP processing of the room temperature deposited ITO thin films was carried out in the PulseForge 3300 system at various power density levels as shown in Fig. 3.17. The sheet resistance value was found to decrease rapidly with an increase in the pulse power density level, and a value below $50 \Omega / \mathrm{Sq}$ was obtained at a power density level of $3.0 \mathrm{~kW} / \mathrm{cm}^{2}$. These results are promising for low temperature contact development for flexible electronic applications.

\subsubsection{Room Temperature ZnO Thin Films}

The room temperature sputtering process was extended to plastic substrates for high performance flexible sensor development. This development provides an alternate path for low-cost, high performance sensor development. Figure 3.18(a) shows the prototype device with $\mathrm{ZnO}$ thin film deposited on the inter-digitated electrode (IDE) pattern on a plastic substrate. The $\mathrm{ZnO}$ thin films were deposited by pulsed dc sputtering at room temperature. As shown in Fig. 3.18(b), the pulsed dc sputtering process was effective in depositing crystalline c-axis oriented films on plastic substrates. The observed results were promising for the integration of multifunctional sensors on plastic substrates.

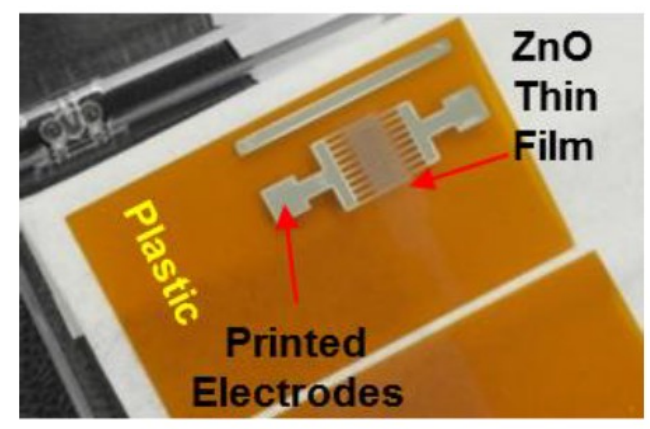

(a)

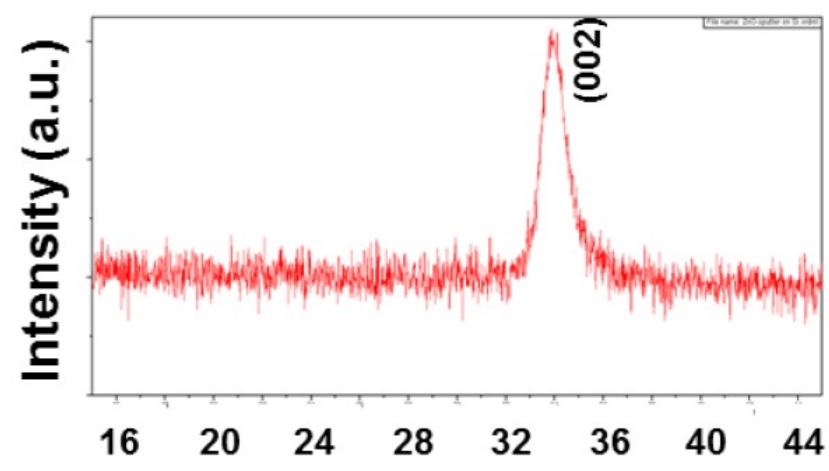

(b)

Figure 3.18. Room temperature deposited $\mathrm{ZnO}$ thin films: (a) thin film on inkjet printed Ag electrode pattern, (b) x-ray diffraction pattern showing c-axis orientation.

The impact of the PTP processing on the crystallinity of the $\mathrm{ZnO}$ thin films was also investigated. Figure 3.19 shows the impact of the PTP processing on the crystallinity of the $\mathrm{ZnO}$ thin films deposited on flexible polyimide (PI) substrates deposited at room temperature. The pulsed dc sputtering process was effective in depositing crystalline c-axis oriented films on plastic substrates. The PTP treatment at a power-density level of $3.0 \mathrm{~kW} / \mathrm{cm}^{2}$ was effective in enhancing the crystallinity of the $\mathrm{ZnO}$ thin films, which is promising for low temperature device integration. Detailed PTP investigation was carried out on $\mathrm{ZnO}$ thin films and devices for low-cost, high performance sensor development.

The impact of the PTP processing on the electrical conductivity of the $\mathrm{ZnO}$ thin films was also analyzed as shown in Fig. 3.20. The current-voltage (I-V) measurements were conducted on ITO/ZnO/PI structures processed at room temperature. The PTP treatment was carried out in the pulse power density range of $1.7-3.0 \mathrm{~kW} / \mathrm{cm}^{2}$ (220-275V applied voltage). The impact of the PTP fluence on the I-V characteristics is clearly evident in Fig. 3.20. The electrical conductivity of the $\mathrm{ZnO}$ thin films was found to increase with an increase in the pulse power density indicating an 
improvement in the material quality, which is consistent with the XRD results showing an improvement in film crystallinity after PTP processing. Detailed PTP investigation were carried out to analyze its impacts on the crystallinity, electrical conductivity, and photo-response characteristics of $\mathrm{ZnO}$ thin films for flexible sensor development.

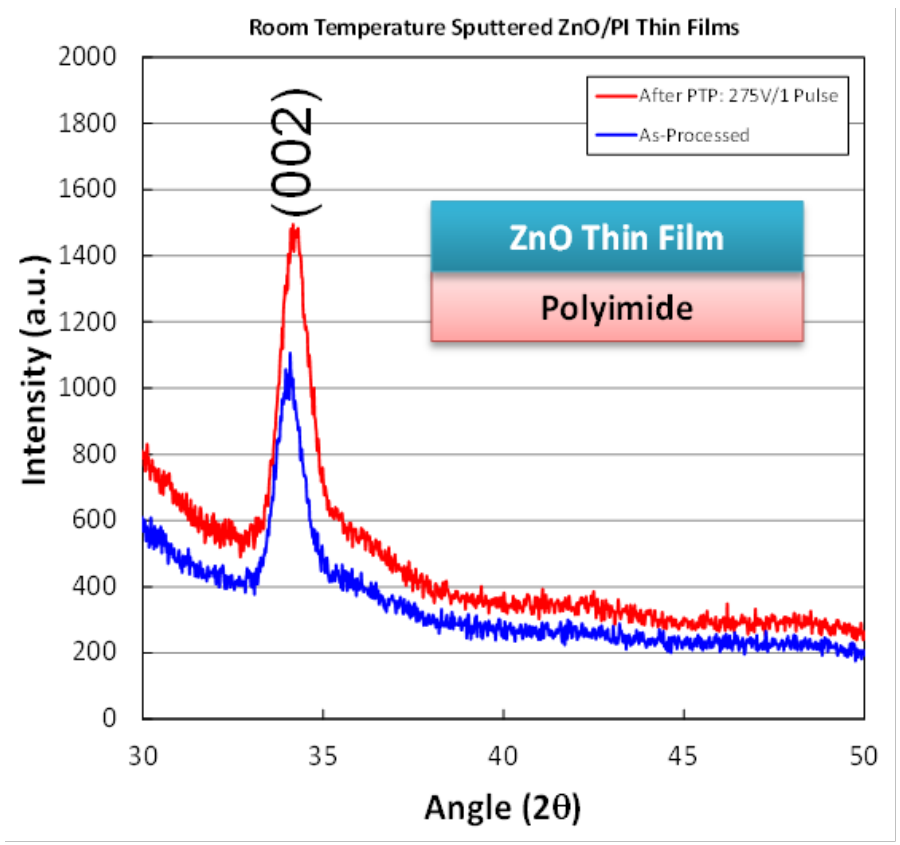

Figure 3.19. The impact of pulse thermal processing on the crystallinity of room temperature processed ZnO thin films.

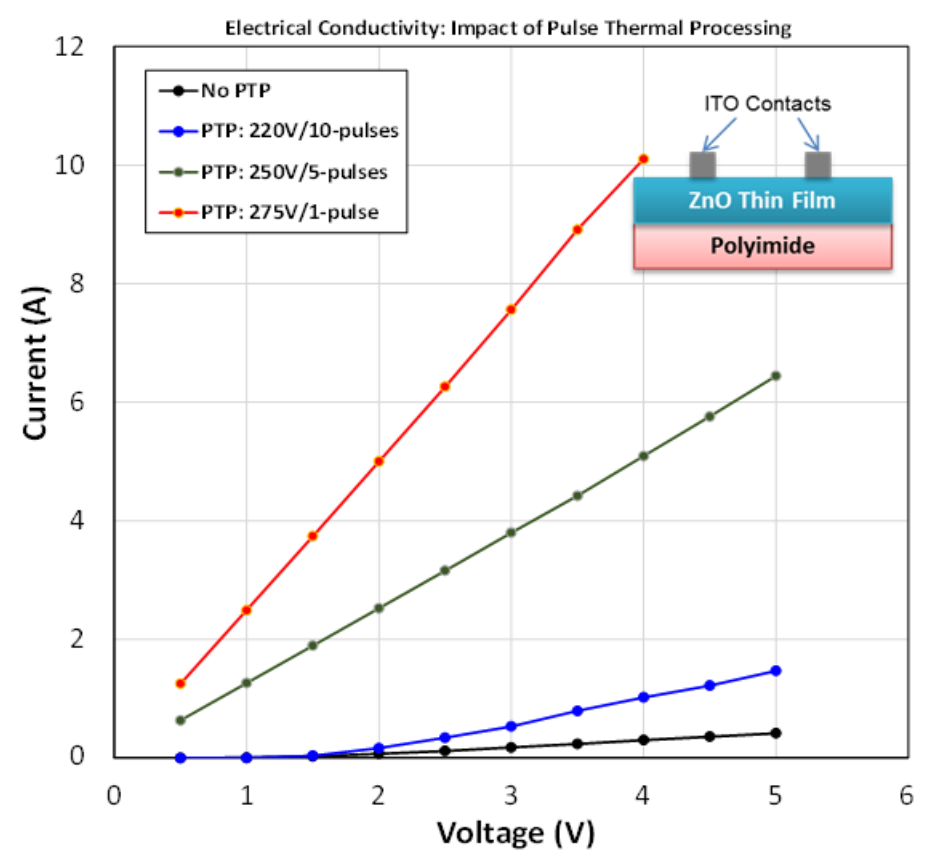

Figure 3.20. The impact of pulse thermal processing on the current-voltage (I-V) characteristics of room temperature processed $\mathrm{ZnO}$ thin films. 

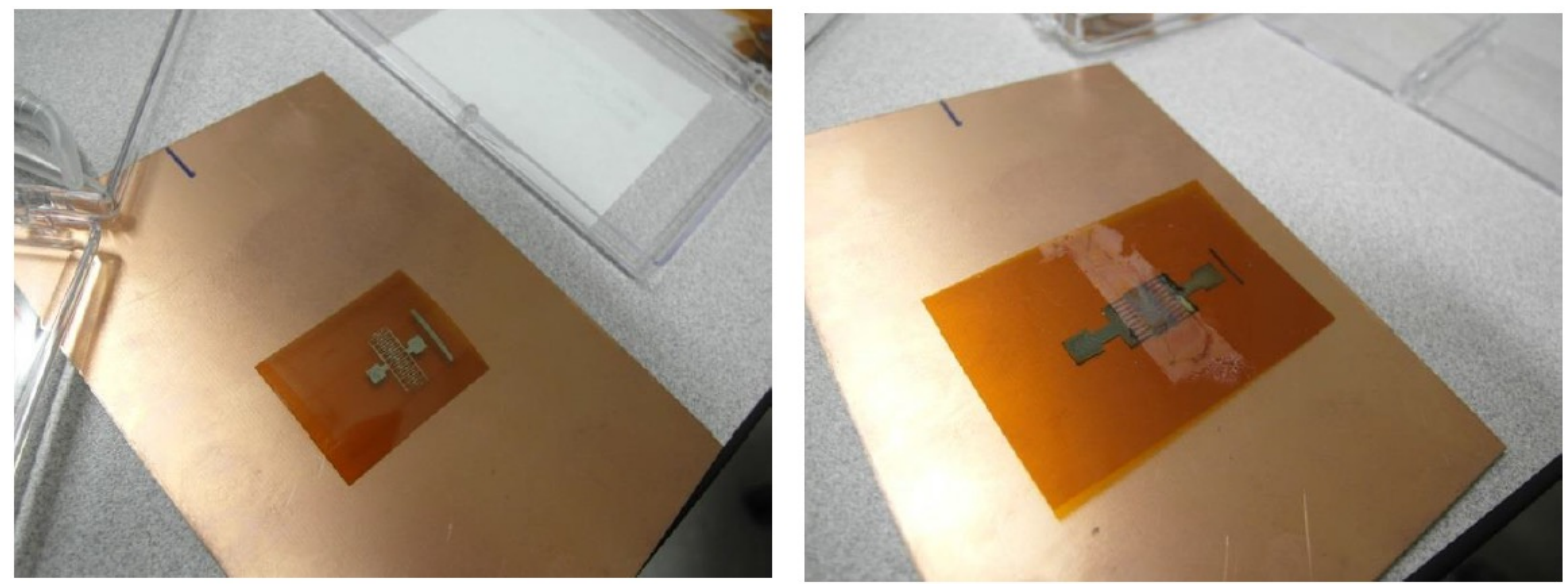

Figure 3.21. UV sensor with interdigitated Ag electrode pattern: (a) electrodes on top, (b) electrodes underneath $\mathrm{ZnO}$ film.

The UV response of the low temperature processed $\mathrm{ZnO}$ thin films on plastic substrates was evaluated using a $365 \mathrm{~nm}$ excitation. The $\mathrm{ZnO}$ films were deposited at room temperature on polyimide substrates by pulsed dc sputtering. The film thickness was maintained at $200 \mathrm{~nm}$. The interdigitated silver electrodes were deposited on various films to evaluate the electrical and photo-response characteristics. Figure 3.21(a) shows a $\mathrm{ZnO}$ sample with inkjet printed Ag electrodes on the top surface of the film. The inkjet printed Ag electrodes had a low sheet resistance of $0.05 \Omega / \mathrm{Sq}$ on polyimide substrates. However, the Ag electrodes on top of the $\mathrm{ZnO}$ films showed high resistivity not suitable for electrical measurements. The observed high resistivity indicated a reaction between the $\mathrm{Ag}$ ink and $\mathrm{ZnO}$ film occurred during the curing process.
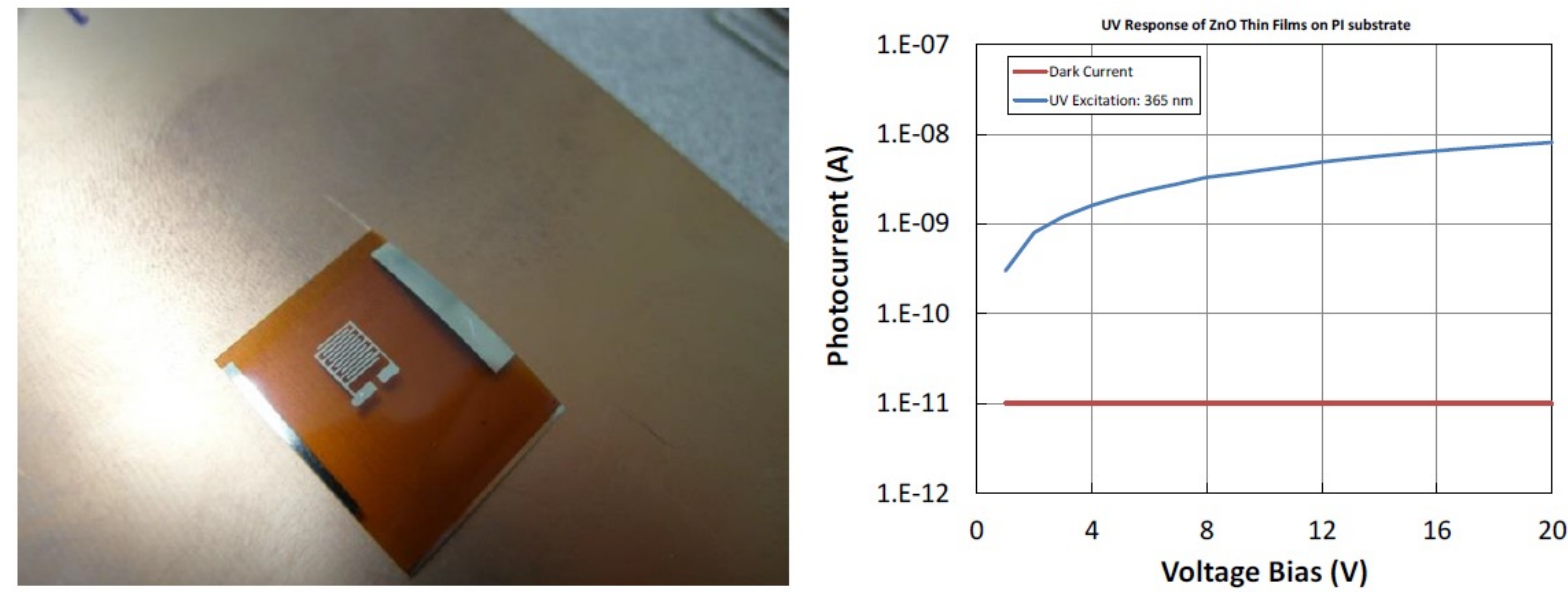

Figure 3.22. (a) ZnO UV sensor with IDE pattern, (b) Photo-response under 365nm illumination.

We attempted the deposition of $\mathrm{ZnO}$ thin film on an interdigitated Ag electrode pattern, as shown in Fig. 3.21(b), which was cured prior to $\mathrm{ZnO}$ deposition. The Ag electrode resistivity did not show any effect of $\mathrm{ZnO}$ deposition in this configuration, indicating the $\mathrm{Ag}$ ink and $\mathrm{ZnO}$ material did react in the top electrode configuration. The UV response of the room temperature deposited $\mathrm{ZnO}$ films was further evaluated in top electrode configuration as shown in Fig. 3.22(a). The top Ag electrodes were deposited by an e-beam evaporation technique at room temperature using a shadow mask. The electrode definition was well maintained on the $\mathrm{ZnO}$ film deposited on a flexible substrate. The UV photo-response of the $\mathrm{ZnO}$ films was evaluated using a $365 \mathrm{~nm} \mathrm{UV}$ excitation. The UV power 
density was $68 \mu \mathrm{W} / \mathrm{cm}^{2}$. As shown in Fig. 3.22(b), the $\mathrm{ZnO}$ films showed a very low dark current in the applied voltage bias range of $0-20 \mathrm{~V}$. It was not possible to measure the absolute dark-current due to the detection limit of the Keithley 2400 SourceMeter. The films showed high photoconductivity under UV excitation. The photocurrent increased by more than 2 orders at a bias voltage of $5 \mathrm{~V}$. The observed results are promising and a show path towards low temperature integration of sensor elements on flexible substrates.

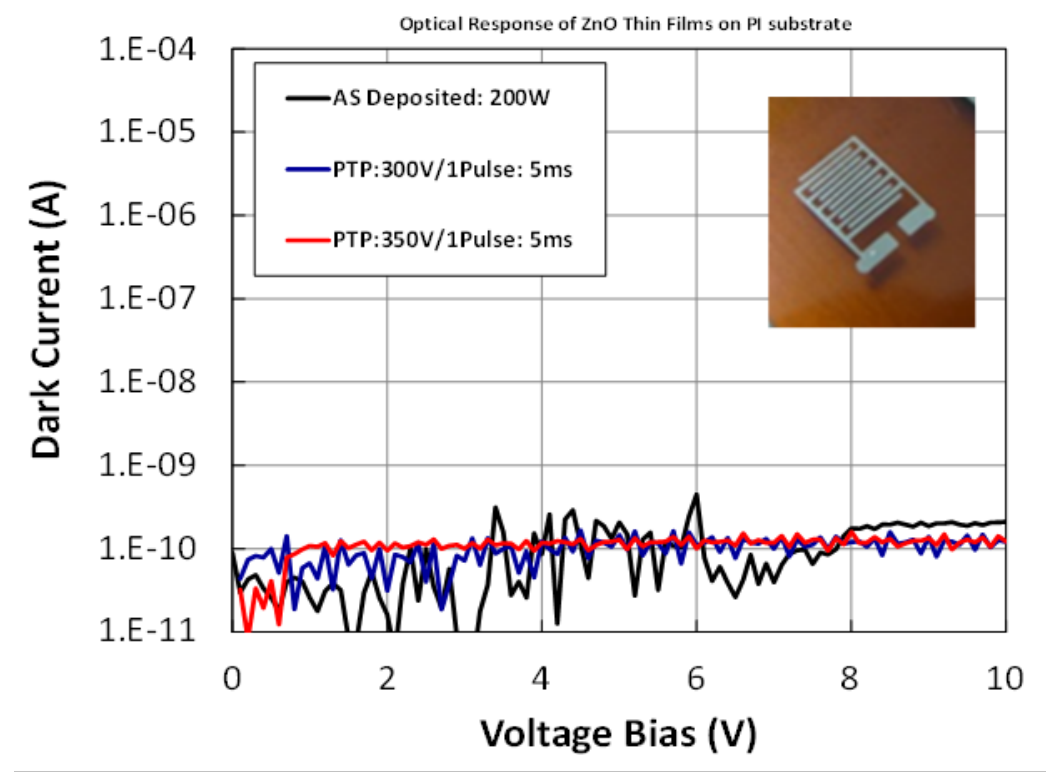

Figure 3.23. The dark-current characteristics of as-deposited and PTP processed ZnO thin films. The films were deposited on polyimide substrates at room temperature.

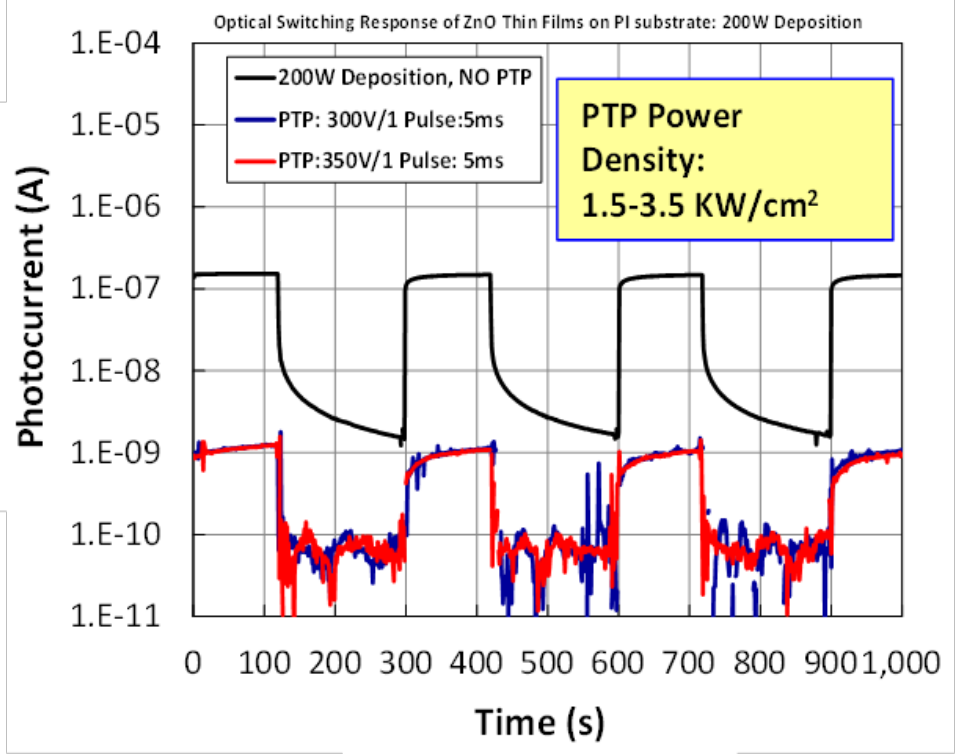

Figure 3.24. The photo response characteristics of as-deposited and PTP processed $\mathrm{ZnO}$ thin films. The films were deposited on polyimide substrates at room temperature.

The next step was to establish the photo- response of the $\mathrm{ZnO}$ thin film based UV sensor as a function 
of PTP processing conditions. Detailed PTP investigation were carried out to analyze its impact on the structural, electrical, and UV photo-response characteristics. ZnO thin films were deposited on polyimide substrates by pulsed dc sputtering at room temperature for flexible UV sensor development. The impact of the pulse thermal processing (PTP) on the dark current and photoresponse of 200-nm-thick films was also analyzed. Figure 3.23 shows the dark-current characteristics of as-deposited and PTP processed $\mathrm{ZnO}$ thin films. The room temperature deposited $\mathrm{ZnO}$ films exhibited low dark-current consistent with the x-ray diffraction results showing high crystallinity.

Figure 3.24 shows the pulsed photo-response of as deposited, and PTP processed $\mathrm{ZnO}$ thin films. The as-deposited films showed more than an order shift in the ON and OFF state current indicating good film quality. The rise time of the photocurrent was found to be much shorter than the decay time under UV excitation. The PTP processing resulted in significant improvement in the switching characteristics as shown in Figure 3.24. Both the rising and trailing edges of the pulsed current showed sharp transitions after PTP processing indicating further improvement in the film's density and crystallinity at a plastic compatible thermal budget. The observed results are promising for the low temperature integration of thin film sensors on flexible substrates using a combination of low temperature deposition processes and low thermal budget pulse thermal processing technique.

\subsubsection{ZnO Particle Coatings}

One effective way to deposit high quality material on low temperature substrates is to decouple the annealing step from the deposition step. High quality $\mathrm{ZnO}$ particles were created by high temperature oxidation of the NF ZnS particles in an oxygen atmosphere. As shown in Fig. 3.25(a), it was possible to achieve a well-crystallized polycrystalline $\mathrm{ZnO}$ phase by oxidation of NF-ZnS particles. The $\mathrm{ZnO}$ coatings were screen-printed on the plastic substrate to analyze their electrical conductivity characteristics.
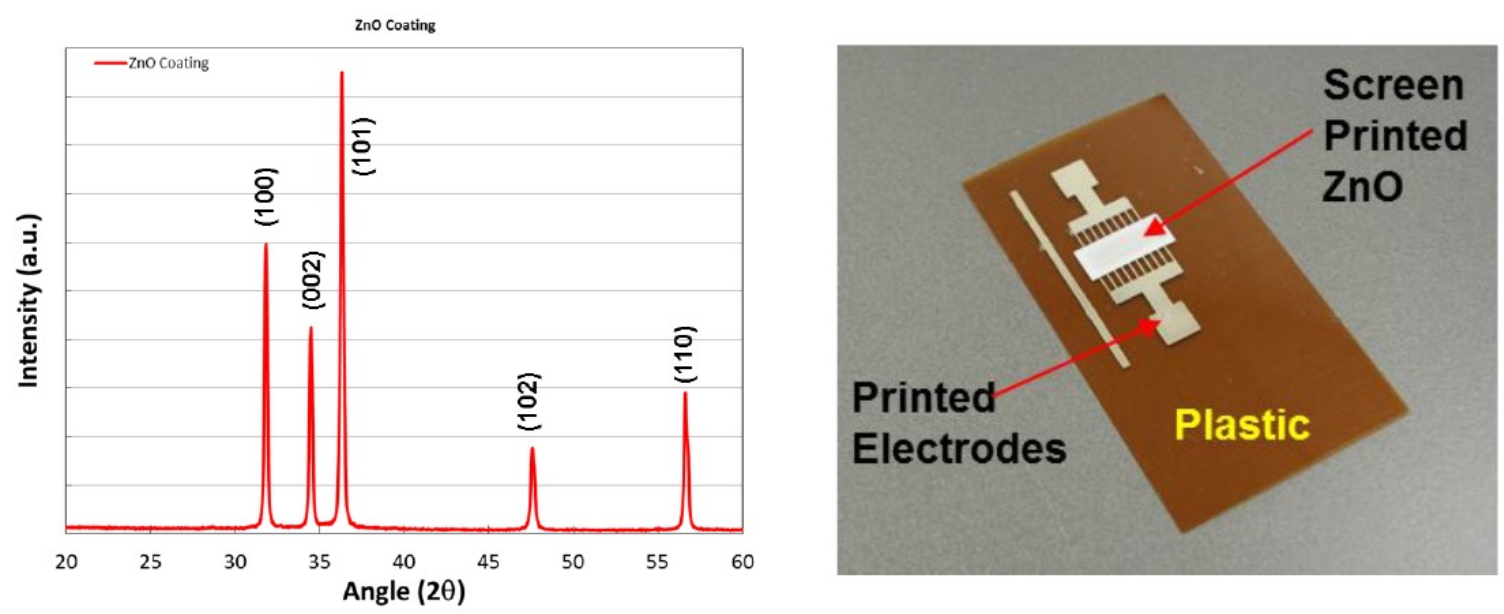

Figure 3.25. Screen-printed $\mathrm{ZnO}$ coatings: (L) x-ray diffraction patterns, (R) coating on inkjet-printed electrode pattern on plastic substrate.

In order to achieve large scale and low cost NF-nanoparticles for functional electro-optical devices the nanoparticles need to be stabilized in order to have the desired properties. As produced NFnanoparticles aggregate (Figure 3.26a) and their solutions are not stable, optically highly scattering. The films are discontinuous and the observed photoluminescence appears to emerge from high energy states (surface defects). When we modified the nanoparticles (Figure 3.27b) with inorganic ligands we found that the solutions were stable, there no measurable light scattering and the optical properties of inorganic-ligated nanoparticles show well-defined band gap and photoluminescence. In addition, 
the NF-nanoparticles tend to self-organize in one-two and three-layer assemblies and show smaller cluster size. In Figure 3.28 we show TEM images of the unmodified NF-nanoparticles cluster (Figure 3.28a) and stabilized NF-nanoparticles clusters Figure 3.28b).

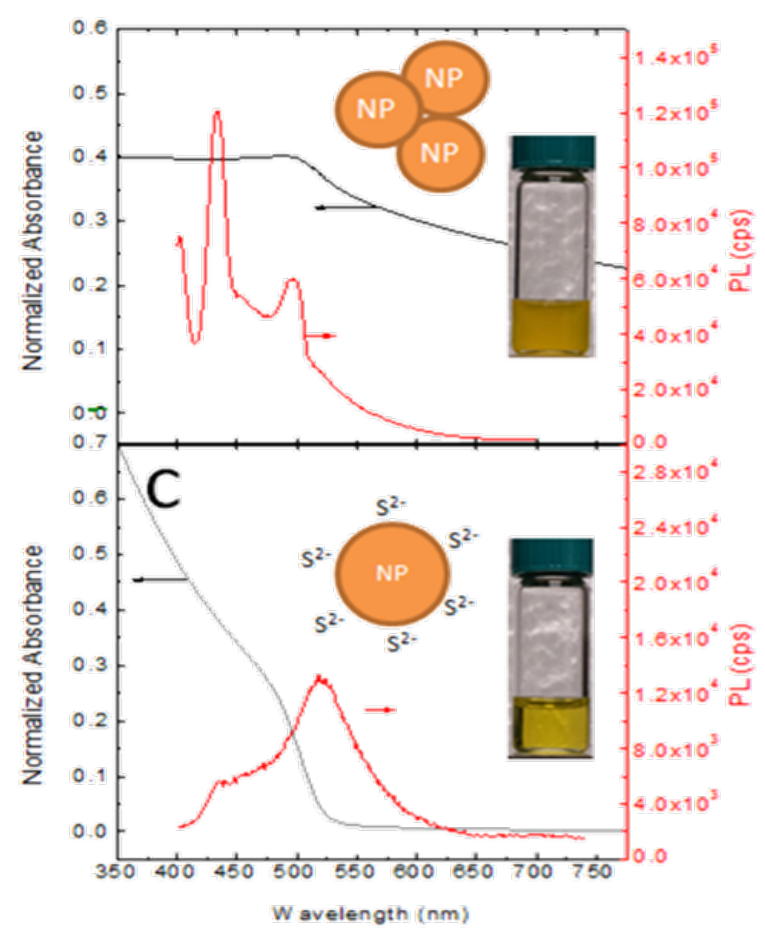

Figure 3.26. (a) Normalized absorbance of as produced NF-nanoparticles. The nanoparticles tend to aggregate, have solutions that are not stable, they are optically scattering and the photo-luminescence comes from high energy states (surface defects). (b) For the stabilized NF- nanoparticles there is no observable light scattering.
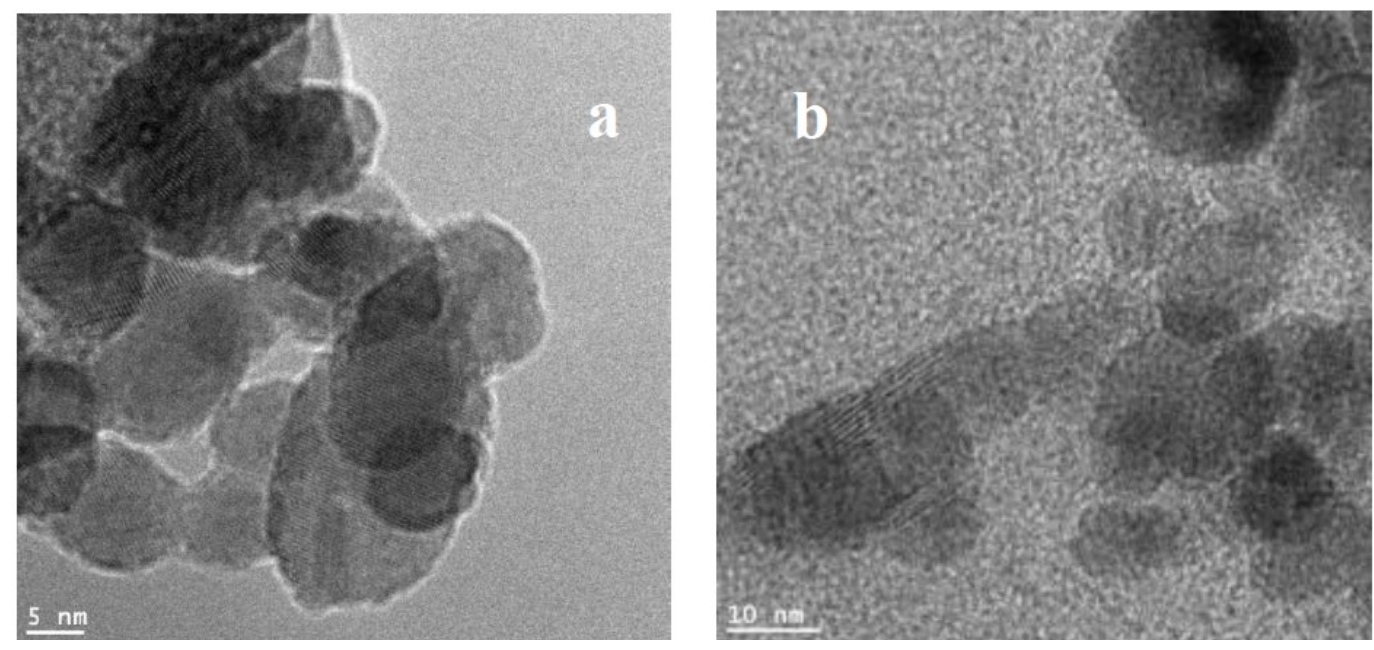

Figure 3.27. TEM images of (a) unmodified and (b) ligand stabilized NF-nanoparticles clusters. 


\subsection{LOW COST METAL OXIDE COATINGS}

As a next step, nanofermented nanoparticles $\left(\mathrm{ZnO}, \mathrm{NiO}, \mathrm{SnO}_{2}\right)$ thin films were deposited on stabilized polyimide substrates to measure the response of the film to humidity and temperature, with impedance measured as a function of frequency for different temperatures. The current as a function of applied voltage was also measured for several samples, as this information is crucial in the efforts to develop low cost sensors.

\subsubsection{ZnO coatings}

The $\mathrm{ZnO}$ coatings were developed on alumina substrates by screen-printing technique. Pt electrodes were also defined by the screen-printing technique. As shown in Figure 3.28, the Pt contact resistance was well-below $1 \Omega$ indicating its suitability for electrical measurements.

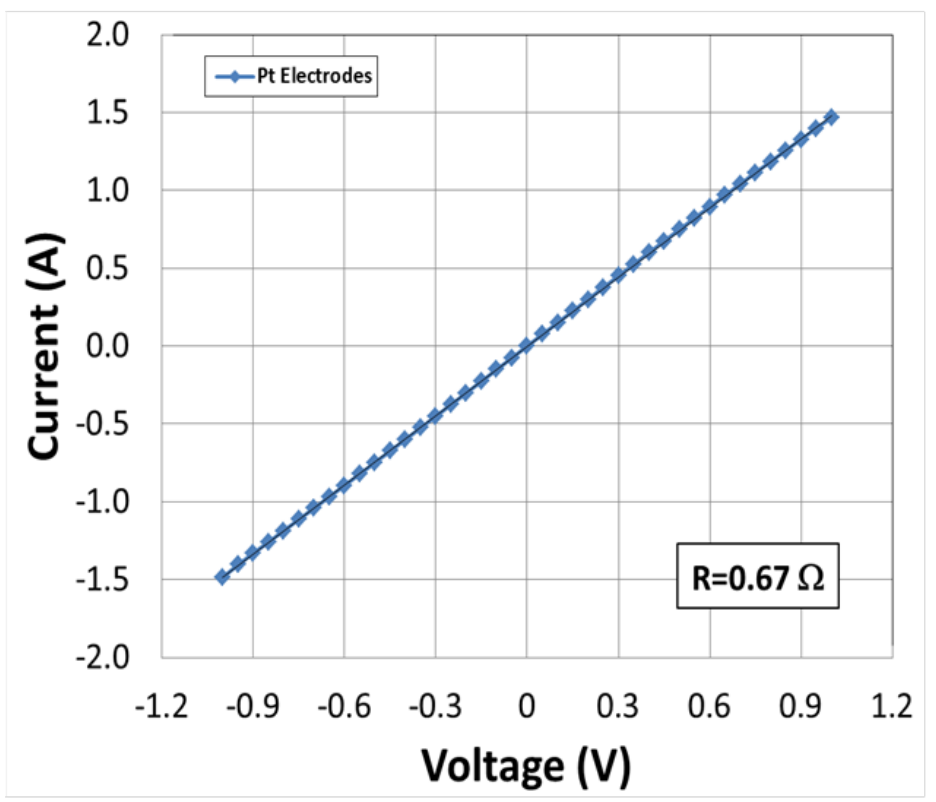

Figure 3.28. Current-voltage (I-V) response of Pt electrodes deposited on alumina substrates.

In order to optimize the line width, we screen printed $\mathrm{ZnO}$ on a polyimide substrate (Figure 3.29). We used this information to screen print $\mathrm{ZnO}$ lines between two Pt pads. This platform formed the basis for a low cost chemical sensor. In Figure 3.30(a) we show an optical image of the printed device. In Figure 3.30(b) we show the surface and depth profile of $\mathrm{ZnO} / \mathrm{Pt} / \mathrm{Al}_{2} \mathrm{O}_{3}$ structures as analyzed by optical microscopy.

The grain growth in $\mathrm{ZnO}$ coatings was analyzed by scanning electron microscope (SEM) as shown in Figure 3.31. Large grain growth was observed in NF particle based $\mathrm{ZnO}$ coatings sintered at 1100 ${ }^{\circ} \mathrm{C}$. The SEM analysis indicated excessive stress in the $\mathrm{ZnO}$ coatings resulting in island formation and discontinuity in parts of the film. The SEM analysis was used to further optimize the chemical composition of the $\mathrm{ZnO}$ inks to improve the coating quality. 


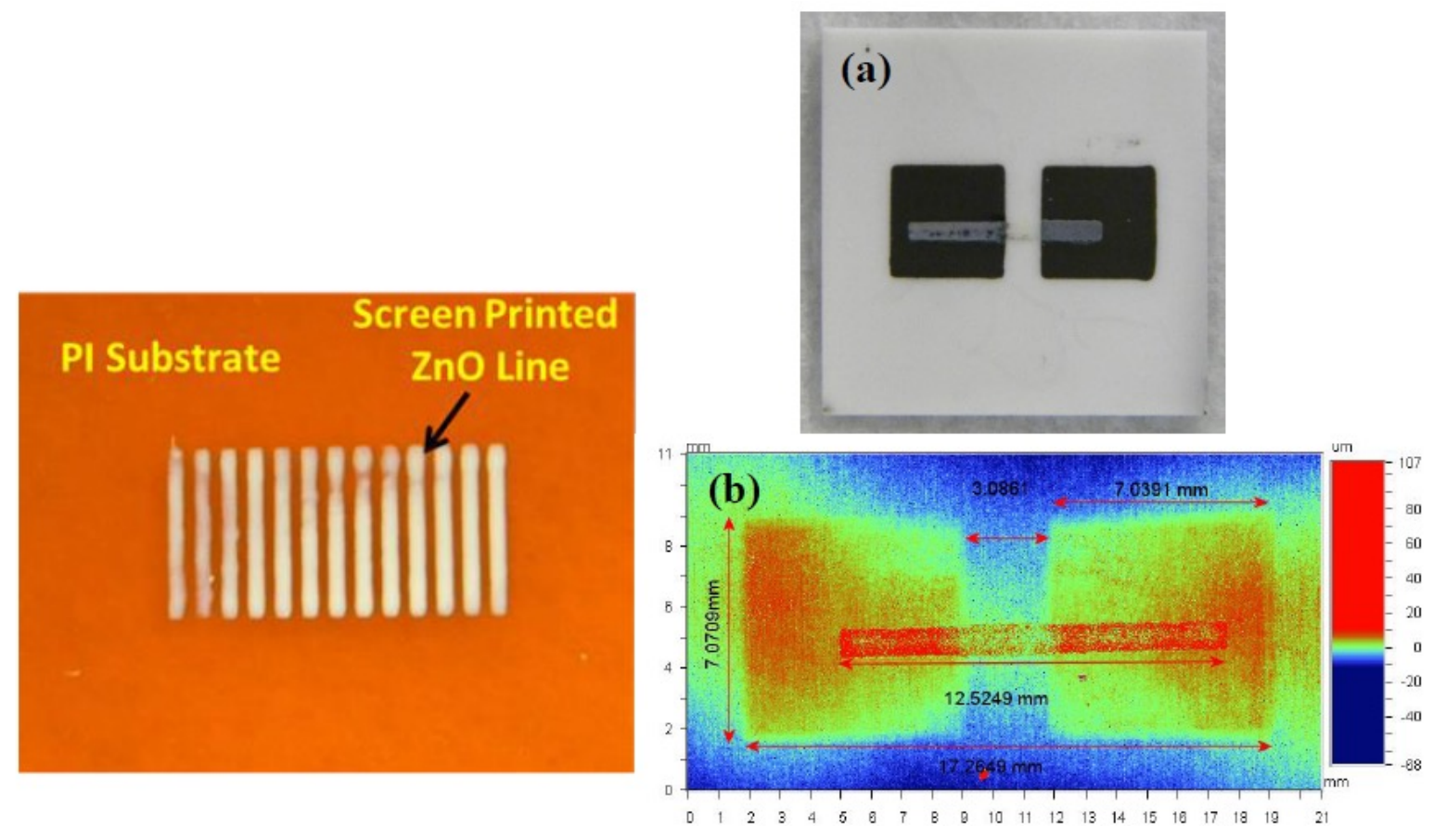

Figure 3.29. Screen printed $\mathrm{ZnO}$ lines.

Figure 3.30. Surface and depth profile of $\mathrm{ZnO} / \mathrm{Pt} / \mathrm{Al2O}$ structures as analyzed by optical microscopy.

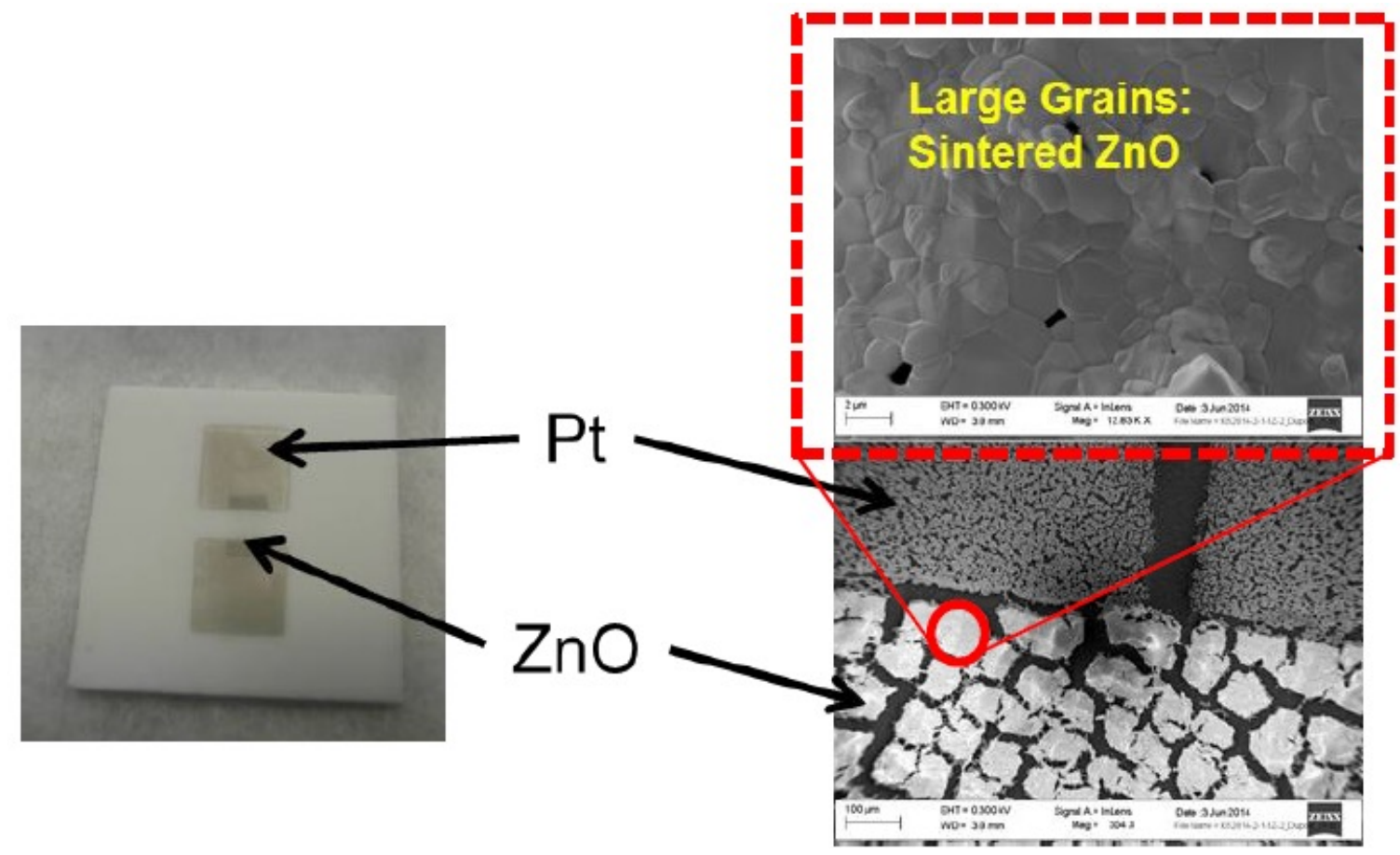

Figure 3.31. A scanning electron micrograph showing large grain growth in NF particle based ZnO coatings.

The electrical conductivity of the $\mathrm{ZnO}$ coatings was evaluated from the current-voltage (I-V) characteristics, as shown Figure 3.32, measured under room temperature conditions. A reference 
$\mathrm{ZnO}$ sample was also prepared using commercial $\mathrm{ZnO}$ particles with an average size of about $10 \mu \mathrm{m}$. The $\mathrm{ZnO}$ coatings sintered below $1100^{\circ} \mathrm{C}$ showed very low conductivity indicating poor grain connectivity and growth. The coatings sintered at $1100^{\circ} \mathrm{C}$ showed a significant improvement in electrical conductivity, however the conductivity-level was about two-orders of magnitude lower than the reference sample. The observed electrical conductivity characteristics indicated the presence of a large concentration of defects in the $\mathrm{ZnO}$ coating even after sintering at high temperatures.

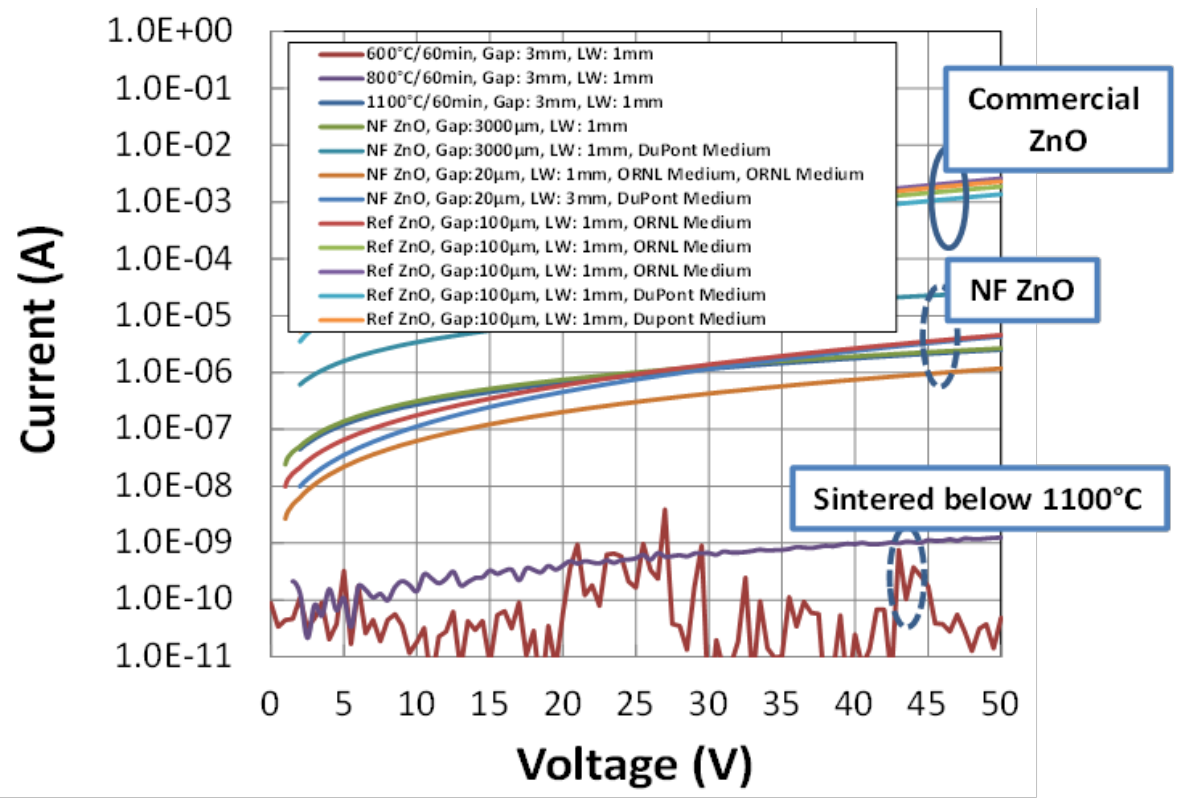

Figure 3.32. Current-voltage (I-V) characteristics of $\mathrm{ZnO}$ coatings sintered at various temperatures.

Forming gas annealing (FGA) was conducted to improve the electrical performance of the $\mathrm{ZnO}$ coatings. As shown in Figure 3.33(a), the electrical conductivity of the $\mathrm{ZnO}$ coatings improved by three-orders of magnitude after FGA, indicating effective passivation of the $\mathrm{ZnO}$ microstructure. A resistivity value of $0.45 \Omega-\mathrm{cm}$ was calculated for these samples, which was suitable for analyzing the gas-sensitivity of the $\mathrm{ZnO}$ coatings. Based on these results, the path towards low-temperature coating development was still not clear, and further reductions in particle size below $200 \mathrm{~nm}$ was critical in reducing the processing temperature for low temperature electronic applications.
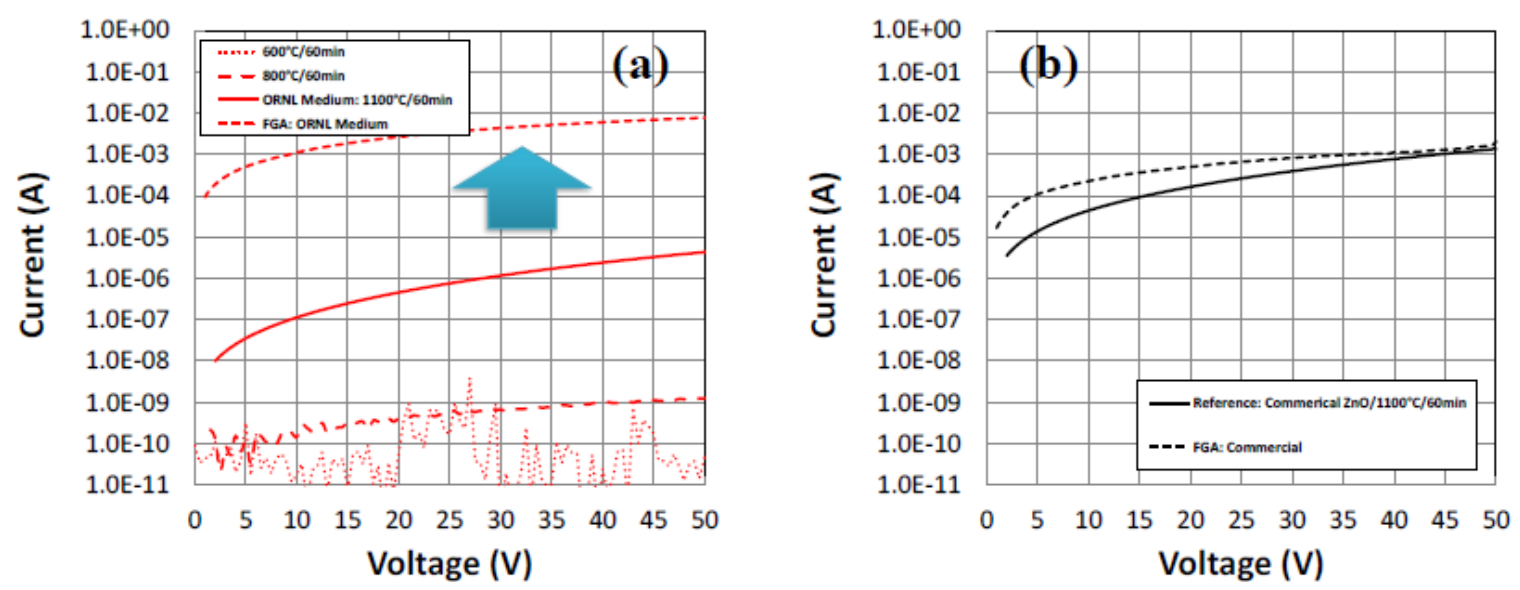

Figure 3.33. Impact of the forming gas annealing on the $I-V$ characteristics of (a) $\mathrm{NF} Z \mathrm{ZO}$ particle coatings and (b) commercial $\mathrm{ZnO}$ particle coatings. 


\subsection{2 $\mathrm{SnO}_{2}$ Coatings}

The $\mathrm{SnO}_{2}$ coatings were also developed for advanced sensor applications. A well-defined crystalline $\mathrm{SnO}_{2}$ phase was observed after annealing at $400^{\circ} \mathrm{C}$. The $\mathrm{SnO}_{2}$ resistor configuration for the electrical measurements is shown in Figure 3.34. This device was annealed at $800^{\circ} \mathrm{C}$ and then at $1000^{\circ} \mathrm{C}$. However, after annealing at $1000^{\circ} \mathrm{C}$ the $\mathrm{Pt}$ pads on the $\mathrm{SnO}_{2}$ layer showed signs of delamination from the substrate, which prevented us from taking the measurements after annealing at $1000^{\circ} \mathrm{C}$ (Figure 3.35). The typical $\mathrm{I}-\mathrm{V}$ characteristic of a coating sintered at $800^{\circ} \mathrm{C}$ is shown in Figure 3.36. The initial results indicate the formation of a highly resistive coating. A further annealing study as a function of ink formulation and NF process conditions was carried out to optimize the coating quality and improve the electrical characteristics.

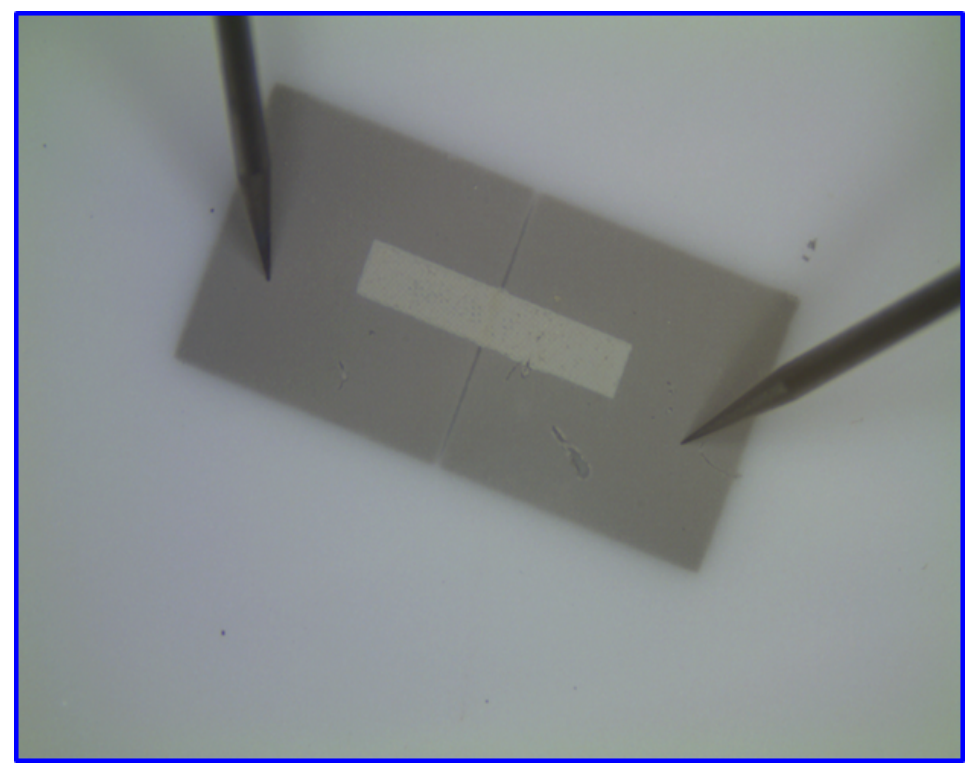

Figure 3.34. $\mathrm{SnO}_{2}$ screen printed between two Pt pads.
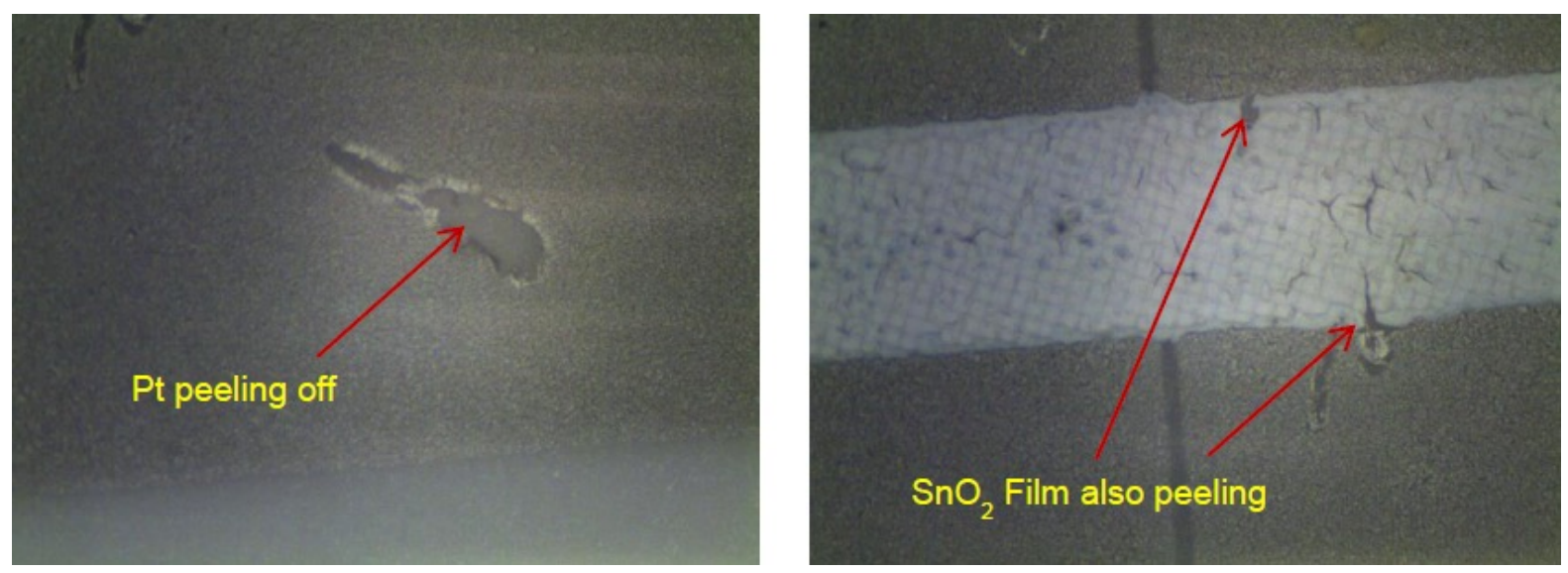

Figure 3.35. Pealing signs of the Pt layer (left) and the $\mathrm{SnO}_{2}$ layer (right) after annealing at $1000^{\circ} \mathrm{C}$. 


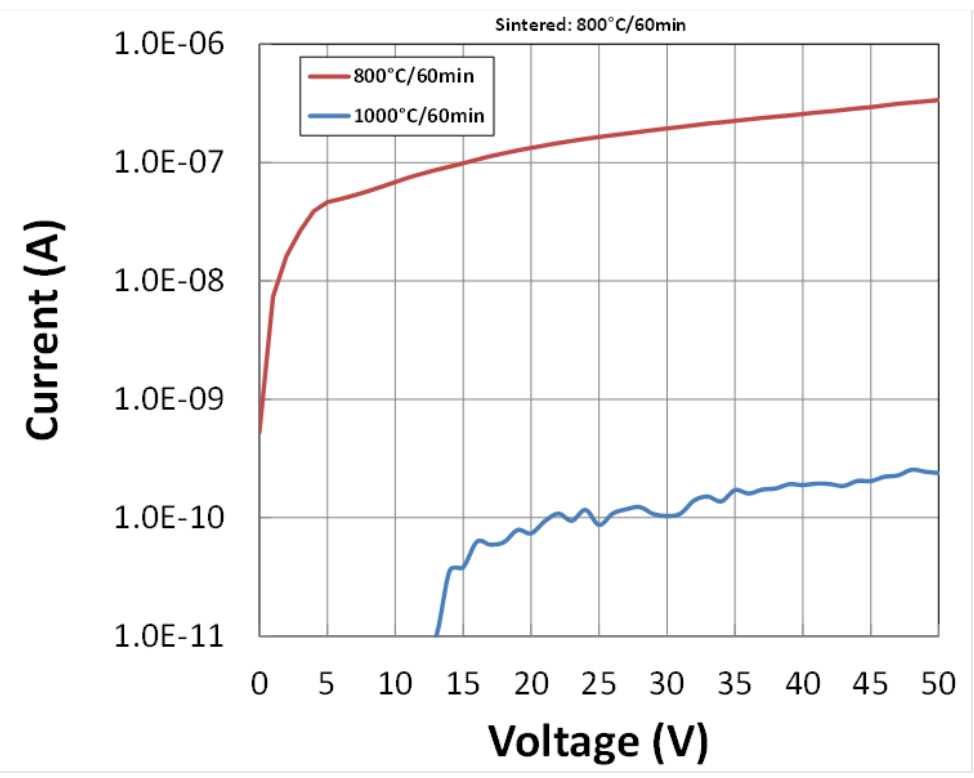

Figure 3.36. Current as a function of applied voltage for two annealing temperatures: $800^{\circ} \mathrm{C}$ and $1,100^{\circ} \mathrm{C}$. Because the film developed discontinuities after annealing at $1000^{\circ} \mathrm{C}$ the conductivity could not be measured.

\subsubsection{NiO Coatings}

The $\mathrm{NiO}$ coatings have also been developed by screen-printing technique on $\mathrm{Pt} / \mathrm{Al}_{2} \mathrm{O}_{3}$ structures using NF particle based inks. The as-processed NF particles were found to be quite large, as shown in Figure 2.14, with average size exceeding $10 \mu \mathrm{m}$. The large size is possibly due to particle agglomeration, which calls for improved chemical processes for effective passivation of particle surface bonds. It was possible to reduce the particle size to the level of $1 \mu \mathrm{m}$, which is still too large to exploit particle size effects for low temperature electronic applications.

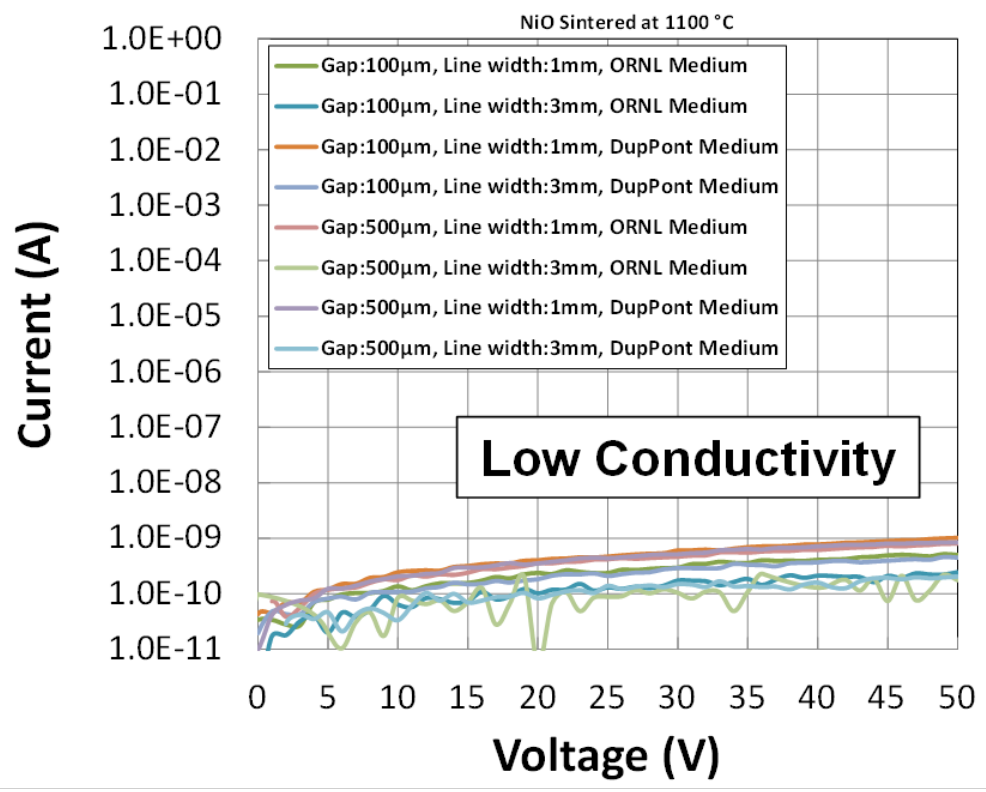

Figure 3.37. Current-voltage (I-V) characteristics of $\mathrm{NiO}$ coatings sintered at $1100^{\circ} \mathrm{C}$. 
The electrical conductivity of the NiO coatings was found to be very low as shown in Figure 3.37. The effects of the NF process and the defect passivation annealing were investigated in detail to gain an insight into the factors controlling the electrical conductivity of $\mathrm{NiO}$ coating. Commercial $\mathrm{NiO}$ particle coatings were developed to establish a reference, and to analyze the key factors influencing the microstructure and electrical characteristics.

\subsubsection{ZnO Sensors on Alumina Substrates}

These metal-oxide-metal structures were employed, using inkjet printing, to demonstrate interdigitized electrode printing, at $100 \mu \mathrm{m}$ line width, on thin film metal-oxide at a low process temperature $\left(<200^{\circ} \mathrm{C}\right)$. Figure 3.38 shows an optical image of a prototype sensor fabricated in this manner, and these structures were used for sensor characterization.

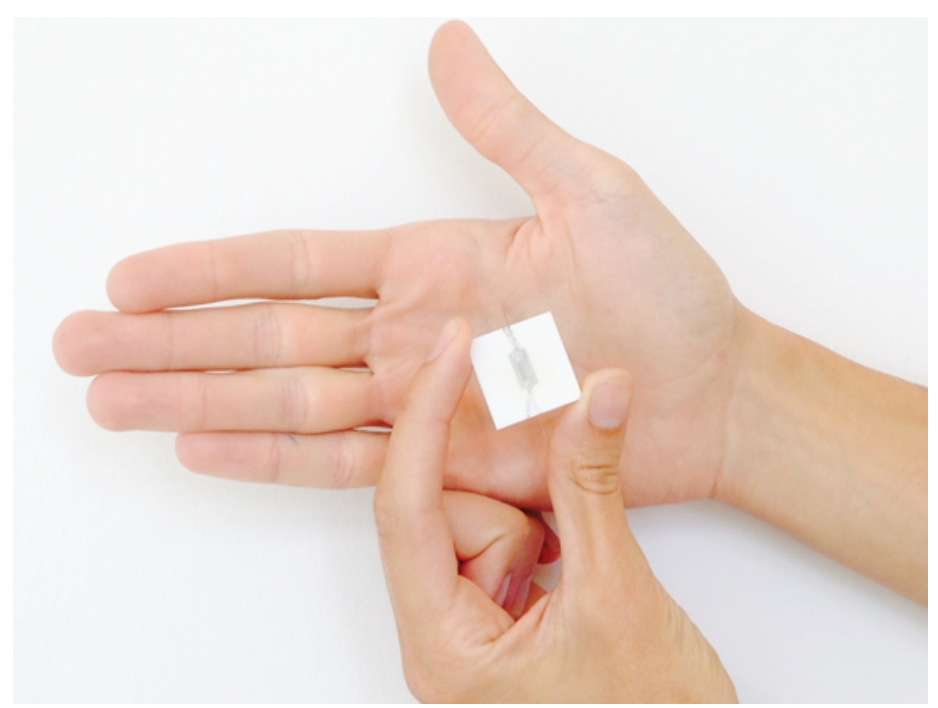

Figure 3.38. $\mathrm{ZnO}$ sensor made from $1 \mathrm{~mm}$ wide strip of screen printed $\mathrm{ZnO}$ on Alumina substrate. The electrodes were screen printed Pt $100 \mu \mathrm{m}$.

The sensors we have developed operate at $0.5 \mathrm{~V}$ compared to $5 \mathrm{~V}$ of conventional sensors. It can be activated using UV light compared to conventional sensors that require $400^{\circ} \mathrm{C}$ for activation. Use of UV light is more energy efficient compared to using a high temperature system (see Figure 3.39(a)). The UV activation process improves sensor response by a factor of five, and the sensor response is directly proportional to the exposure time (amount of material). For the baseline sensor, the impedance was reduced by $55 \mathrm{kOhm}$ under UV illumination, leading to a permanent change, and a $2 \mathrm{x}$ improvement in sensor impedance response to a single pulse. The sensor kinetics were $212 \mathrm{sec}$ with $\mathrm{UV}$ activation and $23 \mathrm{sec}$ partial recovery time $\left(\mathrm{CO}_{2} / \mathrm{H}_{2} \mathrm{O}\right)$. 

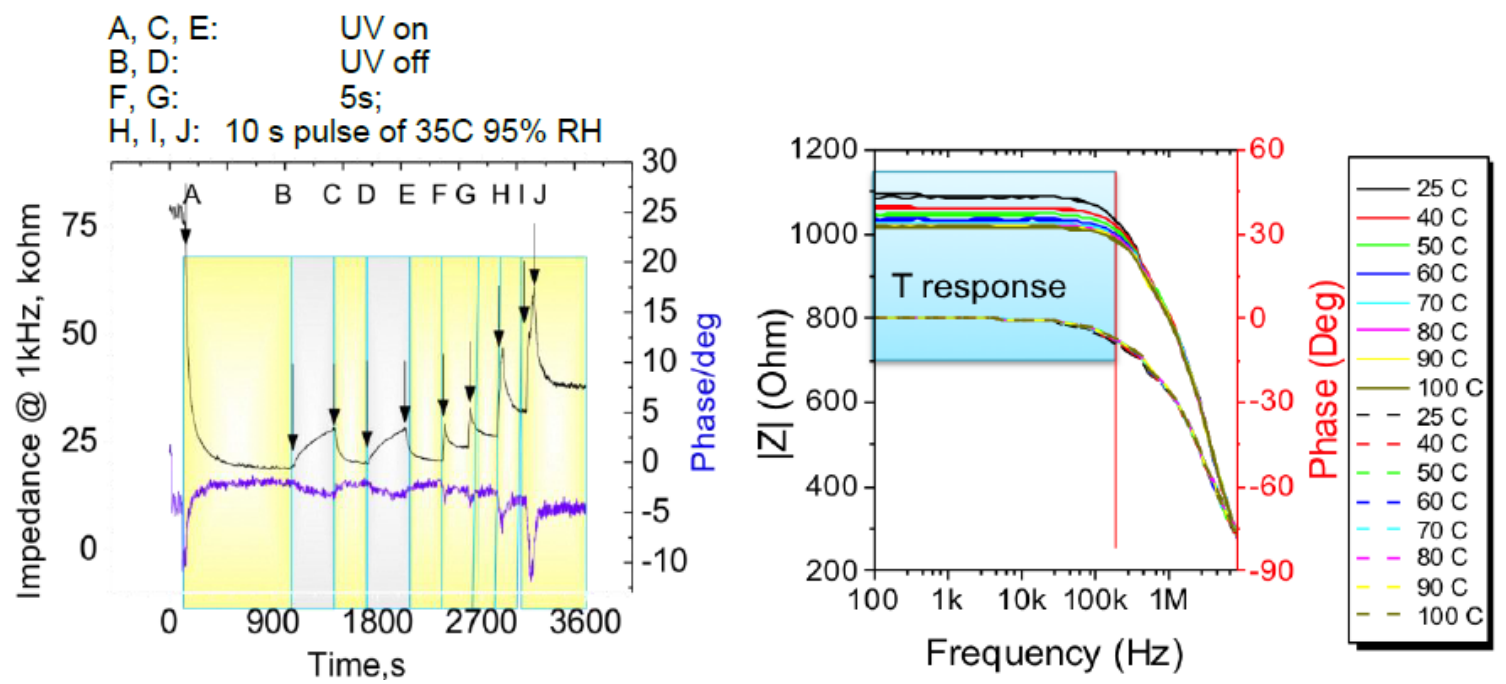

Figure 3.39. Response of $\mathrm{ZnO}$ thin film sensor to relative humidity under $\mathrm{UV}$ activation (a) as a function of UV illumination and (b) as a function of sensor temperature. The humidity response was measured at $35 \mathrm{C}$ and $95 \% \mathrm{RH}$.

The complex impedance decreases as the temperature increases at frequencies $<200 \mathrm{kHz}$ (see Figure 3.39 (b)). The temperature response is resistive up to about $200 \mathrm{kHz}$ and becomes capacitive above $200 \mathrm{kHz}$ on high purity, stabilized $\mathrm{ZnO}$ surfaces. On non-stabilized surfaces the response is capacitive. The response of the $\mathrm{ZnO}$ thin film sensor to temperature was also measured over the range of $25^{\circ} \mathrm{C}$ to $125^{\circ} \mathrm{C}$, and we found the response to be linear (Figure 3.40) as a function of temperature

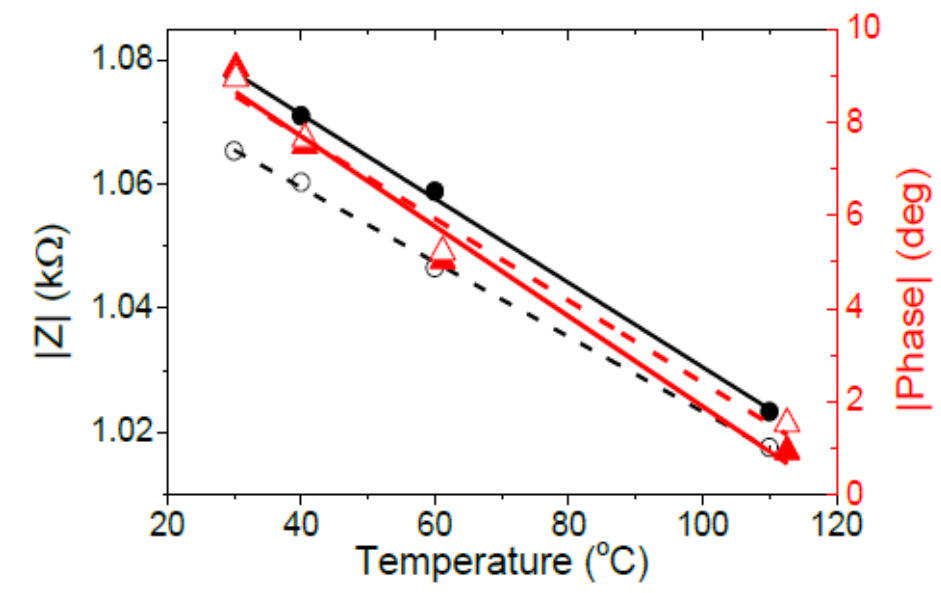

- $\mathrm{RT}|\mathrm{Z}|$ Slope $=-0.67968$

O UV IZI Slone $=-0.60317$

$\Delta$ RT Phase Slope $=-0.01197$

$\triangle$ UV Phase Slone $=-0.01091$

Figure 3.40. Response of $\mathrm{ZnO}$ thin film sensor as a function of temperature.

\subsection{INTEGRATED SENSOR SUITE}

As a final project task, a multi-sensor analysis suite was constructed that was used for the evaluation and testing of the low cost printed sensors that were fabricated using the nanofermented 
nanoparticles. A user interface using LabView was designed and tested. This suite provided us with the ability to simultaneous detect several parameters. The software was modified to accommodate multiple sensors for testing as well as a low cost processor platform. Figure 3.41(a) shows a screen capture of the LabView based multi-channel software. Figure 3.41(b) shows a low cost processor prototype platform for mobile sensing which allows up to 5 sensors inputs, and was developed for implementation and testing of the nanofermented nanoparticles sensors. The readout of these sensors was through a Bluetooth connection.
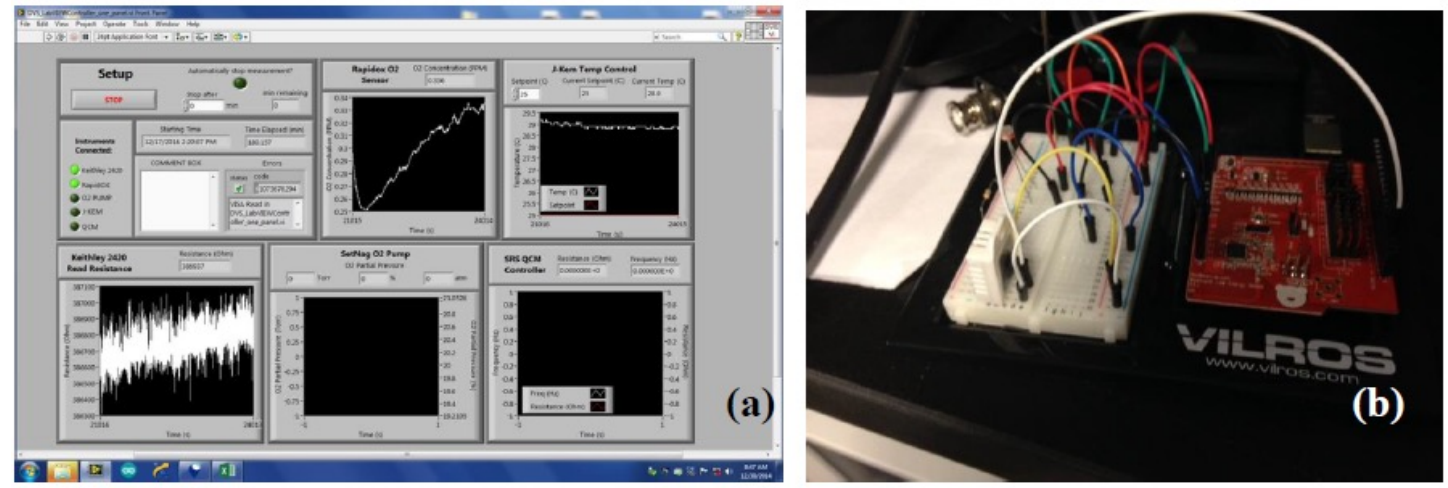

Figure 3.41. (a) Screen capture of the Labview based multi-channel software designed for tests of sensors manufactured from nanofermented nanoparticles, and (b) low cost processor platform for mobile sensing which allows to power up to 5 sensors is being developed for the implementation of the NF sensors.

We explored the effect of substrate temperature on the electrical properties of the $\mathrm{ZnO}$ films. The reference and NF ZnO films displayed similar trends. Namely, the low frequency impedance decreased significantly, by a factor of 4.5 and 6.9 for ref- $\mathrm{ZnO}$ and $\mathrm{NF} \mathrm{ZnO}$ respectively, for samples deposited at elevated temperatures. The frequency dependence of the electrical properties for these samples was also measured. The measurements in Figure 3.42 are presented in the form of Nyquist plot of imaginary and real impedance of $\mathrm{ZnO}$ thin film sensors prepared using pulse laser deposition. The green and blue colored curves are data for reference $\mathrm{ZnO}$ samples and the black and red curves are measurements for the nanoferemented $\mathrm{ZnO}$.

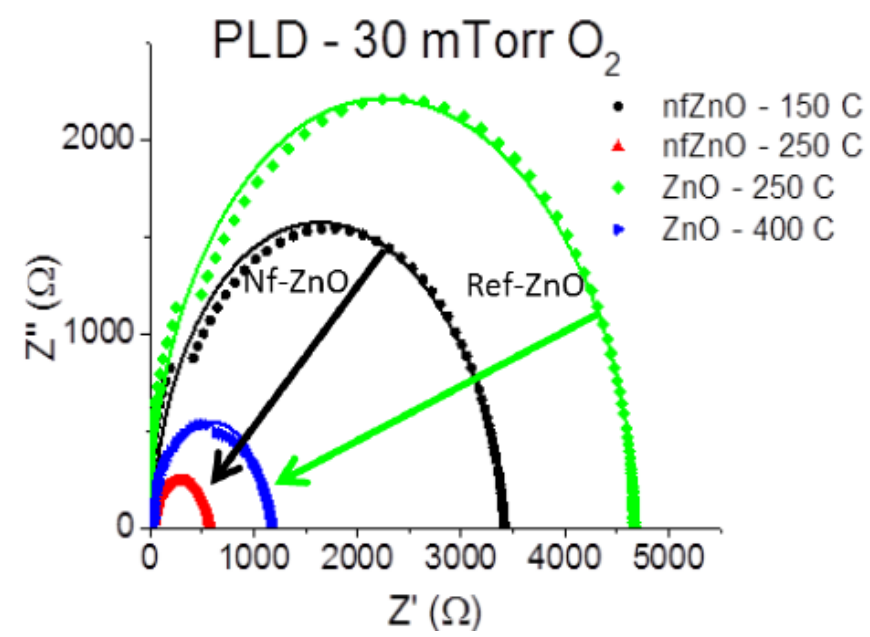

Figure 3.42. Frequency dependent electrical properties (presented in the form of Nyquist plot of imaginary and real impedance) of $\mathrm{ZnO}$ thin film sensors prepared using pulse laser deposition. The green and blue colored curves are data for reference $\mathrm{ZnO}$ samples and the black and red curves are measurements for the nanoferemented $\mathrm{ZnO}$. 
Table 3.3. Summary of the electrical properties of $\mathrm{ZnO}$ films prepared by pulse laser deposition.

\begin{tabular}{|l|l|l|l|l|l|l|l|l|l|}
\hline Target & $\begin{array}{c}\text { Temp } \\
\left({ }^{\circ} \mathrm{C}\right)\end{array}$ & $\begin{array}{c}\text { Pressur } \\
\mathrm{e} 10^{-2} \\
(\text { Torr })\end{array}$ & $\begin{array}{c}\mathrm{Gap} \\
(\mu \mathrm{m})\end{array}$ & $\begin{array}{c}\mathrm{R}_{\mathrm{s}} \\
(\Omega)\end{array}$ & $\begin{array}{c}\mathrm{L}_{1} \\
10^{-6}(\mathrm{~h})\end{array}$ & $\begin{array}{c}\mathrm{R}_{1} \\
(\Omega)\end{array}$ & $\begin{array}{c}\mathrm{C}_{1} \\
(\mathrm{~F})\end{array}$ & $\begin{array}{c}\mathrm{R}_{2} \\
(\Omega)\end{array}$ & $\begin{array}{c}\mathrm{C}_{2} \\
(\mathrm{~F})\end{array}$ \\
\hline $\begin{array}{l}\mathrm{NF} \\
\mathrm{ZnO}\end{array}$ & 250 & 0.001 & 25 & 19.1 & 1.28 & $1.08 \mathrm{E}^{+4}$ & $1.17 \mathrm{E}^{-10}$ & 150.0 & $1.30 \mathrm{E}^{-10}$ \\
\hline $\begin{array}{l}\mathrm{NF} \\
\mathrm{ZnO}\end{array}$ & 150 & & 25 & 6.5 & 7.75 & $1.47 \mathrm{E}^{+3}$ & $1.32 \mathrm{E}^{-9}$ & 449.0 & $3.05 \mathrm{E}^{-6}$ \\
\hline $\mathrm{ZnO}$ & 250 & & 15 & 37.9 & 4.09 & $4.48 \mathrm{E}^{+2}$ & $1.07 \mathrm{E}^{-8}$ & 9.45 & $7.47 \mathrm{E}^{-7}$ \\
\hline & 400 & & 25 & 43.4 & & $2.07 \mathrm{E}^{+3}$ & $2.25 \mathrm{E}^{-10}$ & 197.0 & $1.29 \mathrm{E}^{-7}$ \\
\hline
\end{tabular}

Table 3.3 summarizes the electrical properties of the $\mathrm{ZnO}$ films deposited on the $\mathrm{Si} / \mathrm{SiO}_{2}$ substrates. The deposition was carried at increasing substrate temperatures of $150^{\circ} \mathrm{C}, 250^{\circ} \mathrm{C}$ and $400^{\circ} \mathrm{C}$, and background oxygen pressures of $10^{-5}$ and 0.03 Torr. As the temperature of the substrate increased, the molecules move over longer distances to form a film like coating, whereas at low temperatures the mobility is limited, resulting in columnar structures on the film. The impedance spectra shown in Figure 3.42 were fitted using the model shown in Figure 3.43, which comprised a combination of $\mathrm{R}_{\mathrm{S}}$, $\mathrm{L}_{1}$ and two parallel $\mathrm{RC}$ circuits, $\mathrm{R}_{1}, \mathrm{C}_{1}$ and $\mathrm{R}_{2}, \mathrm{C}_{2}$, connected in series. The physical model of the circuit consists of grain and boundaries of $\mathrm{ZnO}$ with additional contributions due to non-ohmic contact with platinum electrodes. Both resistances and capacitances changed from samples deposited at different temperatures, suggesting that either can be potentially implemented for sensing applications.

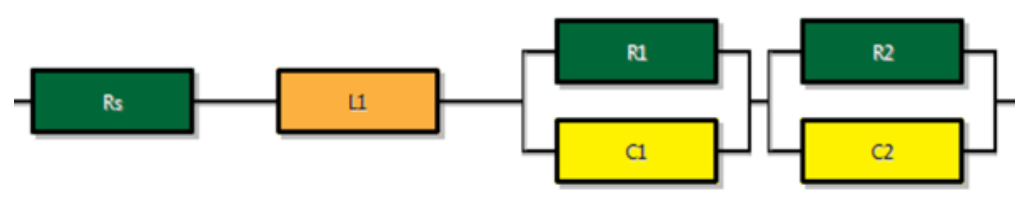

Figure 3.43. Model used to fit the measured data comprised by a combination of $R_{S}, L_{1}$ and two parallel $R C$ circuits, $R_{1}, C_{1}$ and $R_{2}, C_{2}$, connected in series.

The sensing response of screen-printed $\mathrm{ZnO}$ sensor prepared from $\mathrm{ZnS}$ nanoferemented nanoparticles was also measured. The NF nanoparticles were first annealed in the air to convert them to $\mathrm{ZnO}$, and then mixed with ORNL ink carrier for screen-printing. The screen-printing was on the alumina substrates with pre-deposited screen-printed platinum electrodes. After $\mathrm{ZnO}$ sensor line deposition, the sensor was annealed in air. The sensor was exposed to $\mathrm{O}_{2}$ gas and the data collected through the Labview interface shown in Figure 3.41. The results are plotted in Figure 3.44. The black trace shows the unprocessed data for the original resistance the sensor, and the blue trace is the 200 point moving average. The $\mathrm{ZnO}$ sensor response was compared to that of a commercial automotive $\mathrm{O}_{2}$ and its response is shown for comparison with the red trace. We found that that the $\mathrm{NF} \mathrm{ZnO} \mathrm{sensor} \mathrm{was}$ responsive to $<500 \mathrm{ppm}$ of oxygen. 


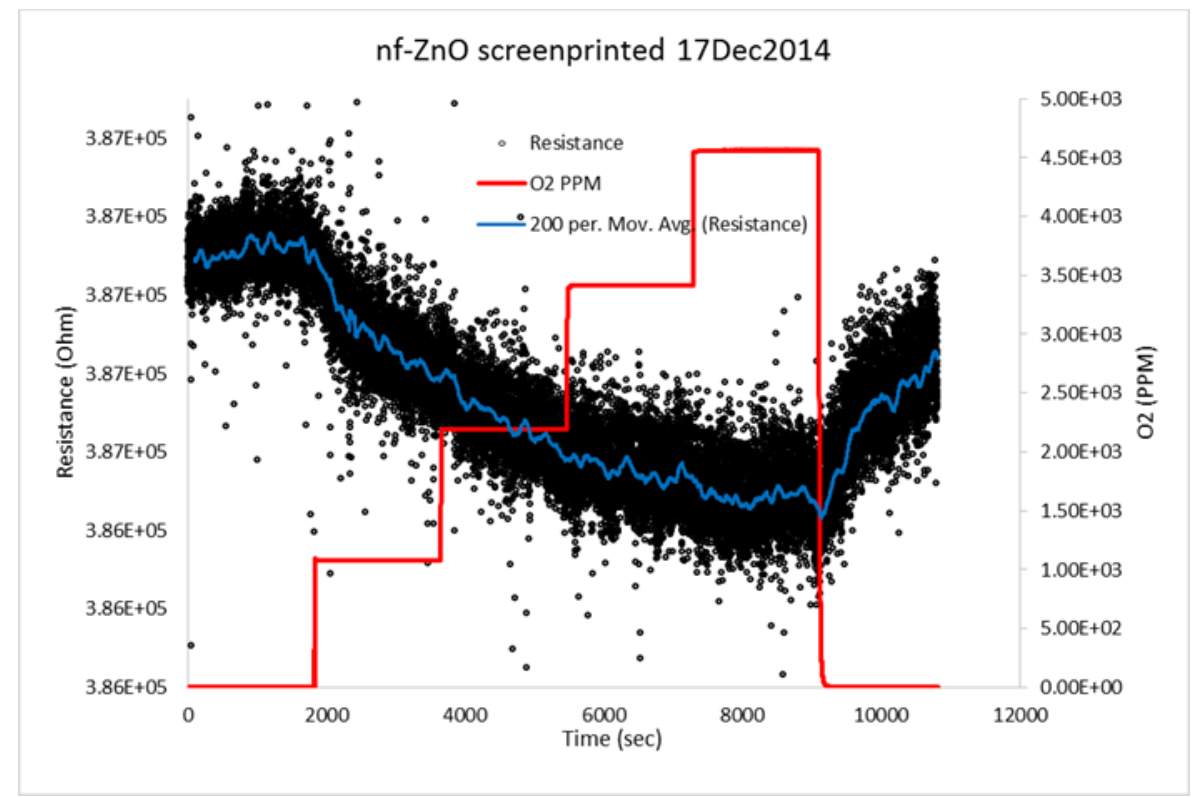

Figure 3.44. Oxygen response of screen printed $\mathrm{ZnO}$ sensor prepared from $\mathrm{ZnS}$ nanoferemented nanoparticles. 


\section{ACCOMPLISHMENTS AND CONCLUSIONS}

The tasks making up this project had several successful outcomes. All the milestones associated with these tasks were successfully accomplished by the conclusion of the project. More specifically we have accomplished the following:

We have demonstrated $\mathrm{Zn}$ based opto-electronic sensors using low cost nanoparticulate structures manufactured using nanofermentation techniques from a related MDF project. We performed photoluminescence measurements and obtained and thin film coatings necessary for the several sensor and electronics applications.

We produced metal sulfide samples $(\mathrm{ZnS}, \mathrm{NiS}, \mathrm{SnS}, \mathrm{CoS}$, and $\mathrm{Cu})$ which were studied over a temperature range in order to determine the optimum heat treatment conditions to convert the sulfide materials to their oxide forms. Using this approach we successfully developed functional metal oxide coatings and evaluated their performance in terms of crystallinity, composition, surface morphology, and mechanical integrity. These nanoparticles are extremely important in many areas of electronics and sensors. Some of the uses will be in p-type and n-type of semiconductor materials which will open up the opportunity for printed roll to roll electronics and sensors.

We used PTP to process high conductivity metallic traces. Silver strain gauges were printed directly on FDM photopolymer substrates. Using this approach we achieved sheet resistance $<50 \mathrm{~m} \Omega / \mathrm{sq}$ for a $1.5 \mu \mathrm{m}$ thick coating or $\sim 5 \mathrm{X}$ silver bulk resistivity for PTP processed, printed silver on porous, rough surfaces such as paper or fibrous materials. We evaluated the use of silver based conductive inks developed by NovaCentrix for electronic device fabrication.

We developed metal-oxide-metal structures employing inkjet printing and demonstrated interdigitized electrode (IDE) printing at $100 \mu \mathrm{m}$ line width on metal-oxide thin film. We demonstrated a suite of low cost printed sensors developed using non-vacuum deposition techniques, which involved the integration of metal, and semiconductor layers to establish a diverse sensor platform technology.

We developed a multi-functional and low-cost resistive sensor integrated onto a flexible substrate and demonstrated low-cost $\mathrm{ZnO}$ sensor integrated for humidity and $\mathrm{O}_{2}$ sensing.

We demonstrated a prototype humidity sensor on plastic substrates and identified an integrated sensor design scheme for multifunctional (gas, chemical, environmental) sensor platform

\subsection{PATENTS}

No new intellectual property was generated during this work.

\subsection{PUBLICATIONS AND PRESENTATIONS}

Publications:

G. Jang, C.B. Jacobs, I.N. Ivanov, Ilia, P. C. Joshi, H. M. Meyer III, M. Kidder, B. L. Armstrong, P.

G. Datskos, D. E. Graham, and Ji-Won Moon, "In Situ Capping for Size Control of

Monochalcogenide (ZnS, CdS and SnS) Nanocrystals Produced by Anaerobic Metal-Reducing

Bacteria," Chem Communications (accepted). 
Presentations:

Chad Duty, "Roll-to-Roll Processing of Flexible Electronics," Next Generation Photovoltaics IUCRC Industrial Advisory Board Meeting." November 11, Knoxville, TN (2013) Invited.

C. Duty, University of Tennessee: Site Update, NSF Industry / University Cooperative Research Program Update (I/U CRC), Fort Collins, CO, July 30, 2013.

Teja Kuruganti, Stephen Killough, Pooran Joshi, "Low-Cost Wireless Sensor Networks for Building Applications Using Novel Materials and Energy-Efficient Communication Techniques," Intelligent Building Operations Workshop, Boulder, CO, June 20-22, 2013.

Teja Kuruganti, Stephen Killough, Pooran Joshi, "Miniature Wireless Sensors as Printable Components," 59th International Instrumentation Symposium, Cleveland, OH, May 13-17, 2013.

C. Duty, Photovoltaic MicroGrid Technology, Defense Manufacturing Conference, Anaheim, CA. November 12, 2012.

P. Joshi, R. Dehoff, C. Duty, W. Peter, R. Ott, L. Love, C. Blue, Direct Digital Additive Manufacturing Technologies: Path Towards Hybrid Integration, Future of Instrumentation International Workshop, Gatlinburg, TN, October 8-9, 2012.

\subsection{COMMERCIALIZATION}

Oak Ridge National Laboratory partnered with Novacentrix of Austin, Texas (printed electronics, nanoparticle manufacturing, pulsed power equipment) on commercialization of these technologies

This project provided advances in roll to roll manufacturing processes of thin film deposition on lowcost flexible substrates for electronics and sensor applications and suggests a promising avenue for the amalgamation of cutting edge nanotechnologies that can be utilized toward DOE's mission fostering opportunities for U.S. companies.

Crucial to the commercialization strategy was the engagement of relevant industry partners (Novacentrix) during the course of the proposed work. We had several interactions with both Cabot Corporation and Novacentrix and have identified areas of future direction that will provide a clear path to commercialization of roll to roll manufacturing processes that include drying/sintering techniques for electrically-conductive inks, and commercially-relevant targets for nanoparticle thin films. In addition, the ORNL technology transfer program will assist in implementing the appropriate mechanism for transferring the technology developed in this work to the commercial arena using IP licensing agreements.

\subsection{RECOMMENDATIONS}

It is recommended that future work focus on several key aspect of the roll to roll approach in order to achieve rapid commercialization and capitalize on existing and emerging market opportunities. The development of non-vacuum, large scale deposition and processing techniques for nanoparticle-based inks and pastes will help reduce cost and energy requirements associated with processing of thin film electronics and sensors. Future efforts should focus on low-cost, flexible substrates, such as polymers and paper and be expanded to include embedded circuitry in additive manufactured components. Additive integration of metal interconnects using inkjet printing of conducting lines is important for 
low-cost flexible electronics. This will define the path towards roll-to-roll processing of multifunctional sensors using low cost $\mathrm{Ag}$ and $\mathrm{Cu}$ inks for interconnects and ORNL's unique pulse thermal processing technology. Furthermore, integration of functionality into structures will allow not only electrical circuits and sensors but provide communication and energy generation / storage capability for a completely autonomous low-cost sensor node. A "traditional" sensor node costs $\$ 150$ while a flexible printed sensor node will be $<\$ 0.15$.

The present work has demonstrated that low cost printed sensors can be fabricated using non-vacuum deposition techniques with the integration of metal and semiconductor layers. This provides a tremendous opportunity to incorporate such capability into additively manufactured products with sensing, energy storage and communications capability. 